Linköping Studies in Science and Technology

Dissertations, No 1603

\title{
Model Based Diagnosis and Supervision of Industrial Gas Turbines
}

\author{
Emil Larsson
}

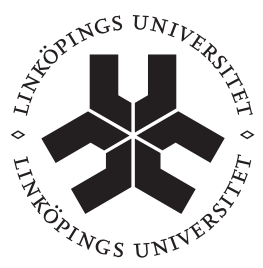

\section{Linköping University} INSTITUTE OF TECHNOLOGY

Department of Electrical Engineering Linköping University, SE-581 83 Linköping, Sweden Linköping 2014 
Linköping Studies in Science and Technology

Dissertations, No 1603

\section{Emil Larsson}

lime@isy.liu.se

www.vehicular.isy.liu.se

Division of Vehicular Systems

Department of Electrical Engineering

Linköping University

SE-581 83 Linköping, Sweden

Copyright ( 2014 Emil Larsson, unless otherwise noted.

All rights reserved.

Larsson, Emil

Model Based Diagnosis and Supervision of Industrial Gas Turbines

ISBN 978-91-7519-312-O

ISSN 0345-7524

Typeset with $\operatorname{ATEX} 2 \varepsilon$

Printed by LiU-Tryck, Linköping, Sweden 2014 


\section{Abstract}

Supervision of performance in gas turbine applications is important in order to achieve: (i) reliable operations, (ii) low heat stress in components, (iii) low fuel consumption, and (iv) efficient overhaul and maintenance. To obtain good diagnosis performance it is important to have tests which are based on models with high accuracy. A main contribution of the thesis is a systematic design procedure to construct a fault detection and isolation (FDI) system which is based on complex nonlinear models. These models are preliminary used for simulation and performance evaluations. Thus, is it possible to use these models also in the FDI-system and which model parts are necessary to consider in the test design? To fulfill the requirement of an automated design procedure, a thermodynamic gas turbine package GTLib is developed. Using the GTLib framework, a gas turbine diagnosis model is constructed where component deterioration is introduced. In the design of the test quantities, equations from the developed diagnosis models are carefully selected. These equations are then used to implement a Constant Gain Extended Kalman filter (CGEKF) based test quantity. The number of equations and variables which the test quantity is based on is significantly reduced compared to the original reference model. The test quantity is used in the FDI-system to supervise the performance and the turbine inlet temperature which is used in the controller. An evaluation is performed using experimental data from a gas turbine site. The case study shows that the designed FDI-system can be used when the decision about a compressor wash is taken. When the FDI-system is augmented with more test quantities it is possible to diagnose sensor and actuator faults at the same time the performance is supervised. Slow varying sensor and actuator bias faults are difficult diagnose since they appear in a similar manner as the performance deterioration, but the FDI-system has the ability to detect these faults. Finally, the proposed model based design procedure can be considered when an FDI-system of an industrial gas turbine is constructed. 



\section{Populärvetenskaplig sammanfattning}

Diagnostik och prestandaövervakning förekommer inom många industriella applikationer. Detta område är viktigt att beakta för att: (i) upprätthålla hög tillförlitlighet, (ii) undvika onödig belastning på komponenter, (iii) minimera energiförbrukningen, och (iv) effektivt kunna planera underhåll. Eftersom prestandan i en applikation oftast inte är direkt mätbar behövs metoder för att kunna skatta dessa prestandaparametrar utifrån kända mätsignaler. Detta kan vara svårt eftersom: (i) sambandet mellan mätsignaler och prestandaparametrar kan vara komplicerat, (ii) mätsignaler innehåller brus, och (iii) mätsignaler kan vara opålitliga och visa ett felaktigt värde. Dessa aspekter bör beaktas när ett diagnos- och övervakningssystem utvecklas. Eftersom många system är komplexa kan det vara nödvändigt att ha effektiva och automatiserade metoder för att skatta prestanda och bestämma diagnoser.

Inom industrin finns det oftast bra modeller som används för att göra prestandaanalyser och simuleringar över olika köruppdrag. För att göra diagnostik- och övervakningsanalyser används ofta andra typer av modeller som är enklare och inte lika beräkningstunga. Dessa två områden är dock nära besläktade och ett gemensamt ramverk skulle kunna användas. Fördelarna med ett gemensamt ramverk är att: (i) endast en modell behöver underhållas, (ii) fel i komponenter och prestandadegraderingar kan bli enklare att modellera eftersom detta kan introduceras i modellen, (iii) diagnostest med en given felkänslighet kan automatiskt genereras, och (iv) diagnostesten kan sedan användas i det utvecklade diagnos- och övervakningssystemet för att bestämma möjliga diagnoser utifrån vilka test som har reagerat.

I denna avhandling presenteras en automatiserad designmetodik för att på ett systematiskt sätt konstruera ett diagnos- och övervakningssystem för en industriell gasturbinapplikation där diagnosbesluten grundar sig på en fysikalisk modell. För industriella gasturbiner är viktiga parametrar att övervaka: (i) verkningsgrader i komponenter, (ii) massflöden genom komponenter, och (iii) temperaturer. En hög gasinloppstemperatur till turbinen kan medföra ökad belastning på materialet vilket leder till en förkortad livslängd. Å andra sidan ger en hög temperatur en bättre verkningsgrad. Eftersom förbränningstemperaturen inte mäts med någon sensor är det viktigt att kunna skatta den så noggrant som möjligt för att inte riskera att överskrida den tillåtna temperaturen. För att bestämma om prestandan har försämrats eller förbättrats är en möjlig lösning att bestämma avvikelsen för dessa parametrar från ett nominellt värde. Detta värde är dock inte konstant utan varierar beroende på arbetspunkt och kan därför beskrivas med en modell för det nominella fallet (vanligtvis den modell som används för prestandaanalyser). Avvikelsen för prestandaparametrarna från nominellt värde sägs, i någon mening, representera gasturbinens hälsotillstånd och kallas därför hälsoparametrar. Dessa hälsoparametrar skattas i de utvecklade diagnostesten.

Det finns ett antal olika typer av industriella gasturbiner med ett brett spektrum vad gäller genererad effekt. De gasturbiner som har högst effekt används ofta för elproduktion i ett kraftverk. Eftersom dessa används för att driva en generator är rotationshastigheten konstant. Ett annat användningsområde är det s.k. mechanical drive vilket är vanligt förekommande inom olje- och gasindustrin. För denna typ av applikation används den genererade effekten för att driva en pump eller en extern kompressor för att exempelvis 
pumpa gas i en rörledning. Flödet i gasledningen kan variera med exempelvis tid på dygnet vilket medför att det är en fördel om det går att variera effekt och rotationshastighet på den påkopplade komponenten. Detta kan uppnås genom att använda en kraftturbin som inte har någon mekanisk koppling med gasgeneratorn som driver kraftturbinen. Eftersom både effekt och rotationshastighet kan varieras bör det ställas högre krav på de modeller som används vilket även fångas upp av den föreslagna designmetodiken.

Avslutningsvis utvärderas diagnos- och övervakningssystemet genom applikationsstudier där data från en gasturbinsite studeras. En av studierna fokuserar på att skatta prestandadegradering vilket kan hänföras till en nedsmutsad kompressor. Övervakningssystemet kan användas som underlag när beslut om att tvätta kompressorn fattas. 


\section{Acknowledgments}

This work has been carried out at the Division of Vehicular Systems at the department of Electrical Engineering, Linköping University. The research has been founded by the Swedish Energy Agency, Siemens Industrial Turbomachinery AB, and GKN Aerospace Sweden AB through the Swedish research program TURBOPOWER, who are gratefully acknowledged.

First of all I would like to express my gratitude to my supervisors Jan Åslund, Erik Frisk, and Lars Eriksson for all their support during these years as a Ph.D. Student at the research group at the Vehicular Systems.

All colleagues at the Vehicular Systems are acknowledged for maintaining a pleasant research atmosphere and interesting discussions during coffee breaks. Christofer Sundström and Daniel Eriksson are both acknowledged for valuable research discussion, especially topics regarding diagnosis and supervision.

Klas Jonshagen, chairman of the Processes and Diagnostics steering committee, is thanked for valuable inputs regarding the work. Mats Sjödin at SIT is thanked for sharing his expertise according to industrial gas turbine applications. Jesper Waldfelt, Lennart Näs, Åsa Lovén, and Christer von Wowern from Siemens Industrial Turbomachinery AB in Finspång are all acknowledged regarding issues according to measurement data and developed models by the company.

Finally, I would like to express my gratitude to Marie for her support and encouragement.

Emil Larsson

Linköping, May 2014 



\section{Contents}

I Introduction 1

1 Background and Motivation 3

1.1 Problem Statement . . . . . . . . . . . . . . . . . . . . . . 4

1.2 Thesis Outline ...................... 5

2 Industrial Gas Turbine Background and Simulation Environment 7

2.1 Gas Turbine Theory . . . . . . . . . . . . . . . . 7

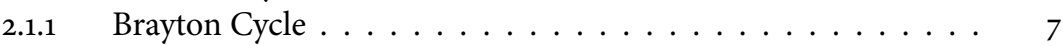

2.1.2 Mechanical Drive Application . . . . . . . . . . . . 8

2.2 SGT-7oo Gas Turbine . . . . . . . . . . . . . . . . . 10

2.2.1 Measurement Signals . . . . . . . . . . . . . . . . . . . . . . . . 10

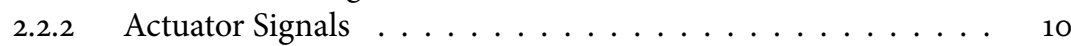

2.3 Simulation Platform . . . . . . . . . . . . . . . 12

2.3.1 Reference Gas Turbine Model . . . . . . . . . . . . . . . . . . 14

$2.3 .2 \quad$ Modelica . . . . . . . . . . . . . . . . . . . 14

3 Summary of Contributions 17

3.1 Thesis Summary . . . . . . . . . . . . . . . . . . . . . . . . . . . . . . . 17

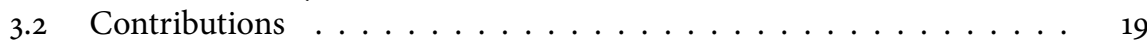

3.3 Publications...................... 21

II Modeling and Design $\quad 23$

4 Thermodynamic Concepts 25

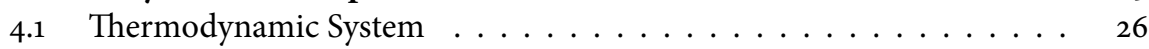

4.1.1 Thermodynamic Quantities . . . . . . . . . . . . . . 26

4.1 .2 Thermodynamic Laws . . . . . . . . . . . . . . . . . 28

4.2 Thermodynamic Properties of Pure Substances . . . . . . . . . . . . . . . . 29

4.2.1 Specific Heat Capacity of Pure Substances . . . . . . . . . . . . . 31

4.2.2 Standardized Enthalpy of Pure Substances . . . . . . . . . . . 31

4.2.3 Standardized Entropy of Pure Substances . . . . . . . . . . . 32 
4.2.4 Gibbs Free Energy . . . . . . . . . . . . . . . . . 32

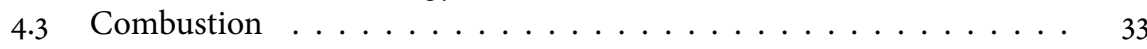

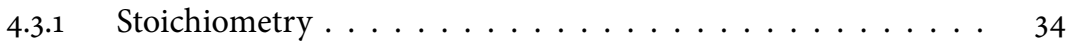

4.3 .2 Chemical Equilibrium . . . . . . . . . . . . . . 37

4.3.3 Comparison of the Heat Capacity Between the Stoichiometric Gas Description and the Chemical Equilibrium Calculation . . 40

4.3.4 Mixing of Exhaust Gases with Different Lambda . . . . . . . 40

4.4 Ideal Gas Model . . . . . . . . . . . . . . . . . . . . . 42

4.4.1 Thermodynamics Properties of Frozen Mixtures . . . . . . . . . . 44

4.5 Energy Conservation of Thermodynamic Systems . . . . . . . . . . . . 44

4.5.1 Thermodynamic Differentials $d U, d W$, and $d Q \ldots \ldots . \ldots 44$

4.5.2 Energy of the Mixture of Frozen Ideal Gases . . . . . . . . . . . . . . 45

4.6 Control Volume Model . . . . . . . . . . . . . . . . . 46

4.6.1 Differential Form of the Ideal Gas Law . . . . . . . . . . . . . . 46

4.6.2 Lambda Concentration Differential $d \lambda \ldots \ldots . \ldots . . . . . .48$

4.6.3 Partial Derivatives of Gas Property Functions . . . . . . . . . . . 48

4.6 .4 State Equations . . . . . . . . . . . . . . . . . 49

4.6.5 Variation in Ambient Absolute Humidity . . . . . . . . . . . . . . 49

4.7 Conclusion ........................ 51

5 GTLib - Thermodynamic Gas Turbine Modeling Package 53

5.1 Gas Turbine Performance Characteristics . . . . . . . . . . . . . . 54

5.1 .1 Compressor Map . . . . . . . . . . . . . . . . . . 55

$5.1 .2 \quad$ Turbine Map . . . . . . . . . . . . . . 56

5.2 Variation in Ambient Absolute Humidity . . . . . . . . . . . . . . . . 57

5.3 GTLib Components . . . . . . . . . . . . . . . . . . . 58

$5.3 .1 \quad$ Global Environment Model . . . . . . . . . . . . . . . 58

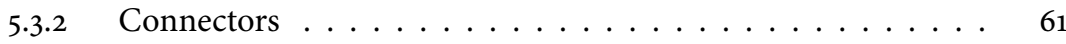

5.3 .3 Gas Model . . . . . . . . . . . . . . . . . . . . 62

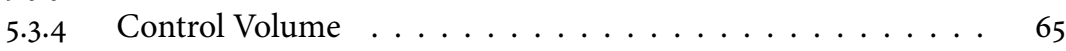

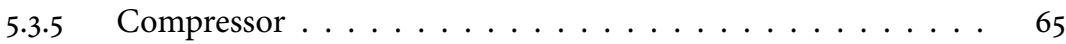

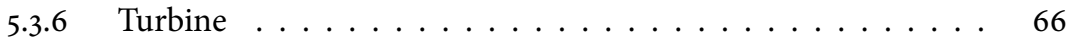

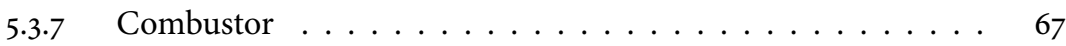

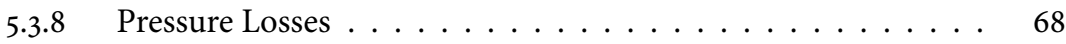

5.4 Gas Turbine Model . . . . . . . . . . . . . . . . . 68

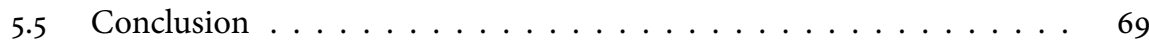

6 Modeling, Analysis, and Transformation of the Diagnosis Model 71

6.1 Gas Turbine Monitoring . . . . . . . . . . . . . . . . 73

6.1.1 Gas Path Analysis . . . . . . . . . . . . . . . . 74

6.1.2 Engine Health Monitoring . . . . . . . . . . . . 75

6.2 Gas Turbine Diagnosis Model . . . . . . . . . . . . . . 76

$6.2 .1 \quad$ Input Signals $\ldots \ldots \ldots \ldots \ldots \ldots$

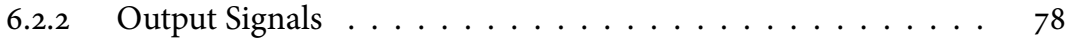

6.2.3 Health Parameters . . . . . . . . . . . . 78 
6.2 .4 Component Faults . . . . . . . . . . . . . . . . . . 79

6.2 .5 Faults in Signals . . . . . . . . . . . . . . . . . 79

6.2.6 Differential Algebraic Equation Form . . . . . . . . . . . 80

6.3 DAE-Index Analysis . . . . . . . . . . . . . . . . . . . . 80

6.3.1 Differential Index Reduction . . . . . . . . . . . . . . . . . . 81

6.4 Structural Analysis . . . . . . . . . . . . . . . . . 82

6.4.1 Dulmage-Mendelsohn Decomposition . . . . . . . . . . 83

6.4.2 Investigation of Actuator Fault and Health Parameter Isolation 85

6.4.3 DAE-index 1 Conservation in the Over-Determined $M^{+}$Part . 87

6.4 Diagnosis Test Equation . . . . . . . . . . . . . . . . 90

6.5 Observability Analysis . . . . . . . . . . . . . . . . 90

6.5.1 Structural Observability . . . . . . . . . . . . . . . . . 91

6.5.2 Removing of Unobservable Modes . . . . . . . . . . . . . . . 92

6.5.3 Number of Health Parameters in the Model . . . . . . . . . . 93

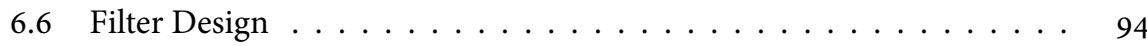

6.6 .1 Kalman Filter . . . . . . . . . . . . . . . . . . . . . 95

6.6 .2 Stationary Kalman Filters . . . . . . . . . . . . . . . . . 96

6.6.3 Nonlinear Kalman Filters . . . . . . . . . . . . . . . . . . . . 96

6.7 Parsers for an Automatic Extraction of Sub Systems _ . . . . . . . . . . . . 97

6.7.1 Automatic Extraction of the DAE Model . . . . . . . . . . . . . . . . 97

$6.7 .2 \quad$ Structural Model Parser . . . . . . . . . . . . . . . . . . 98

6.7 .3 Index Reduction Parser . . . . . . . . . . . . . . . . . 98

6.7.4 State Space Form Construction Parser . . . . . . . . . . . . . . . 99

6.8 Conclusion . . . . . . . . . . . . . . . . . . . . . 101

\section{Application of Methodology 103}

7 Performance Estimation in Industrial Gas Turbine Engines 105

$7.1 \quad$ Background . . . . . . . . . . . . . . . . 106

7.1.1 Experiment Setup . . . . . . . . . . . . . . . . . . 107

7.2 Introductory Methods To Detect Compressor Fouling . . . . . . . . . . 110

7.2.1 Bell-Mouth Based Estimation . . . . . . . . . . . . . 111

7.2.2 Pressure Ratio Based Mass Flow Estimation . . . . . . . . . . . 112

7.2.3 Power versus Mass Flow of Fuel . . . . . . . . . . . . . . . . 112

7.2.4 Performance Model Based Mass Flow Estimation . . . . . . . . 112

7.3 Measurement Delta . . . . . . . . . . . . . . . . . . . . . . . . 114

7.4 Constant Gain Extended Kalman Filter . . . . . . . . . . . . . . . . . . . . . . . . . . . . . . . . . . . 117

7.4 .1 Observability . . . . . . . . . . . . . . . . . . . . . 117

7.4 .2 Observer Tuning . . . . . . . . . . . . . . . . . . . . . . . . 119

7.4 .3 Filter Design Summary . . . . . . . . . . . . . . . . . . . . . . . . . . . . .

7.5 Case Studies . . . . . . . . . . . . . . . . . . . 121

7.5.1 Evaluation 1: Atmospheric Weather Condition Dependence . . 121

7.5.2 Evaluation 2: State Estimation for Control . . . . . . . . . . . 122

7.5.3 Evaluation 3: State Estimation for Monitoring . . . . . . . . . . 124 
7.5.4 Discussion of the Results in Evaluation 2-3 . . . . . . . . . 127

7.5.5 Nonlinear versus Linear Estimator . . . . . . . . . . . . . . . . . 127

7.6 Summary of the Performance Estimation Techniques . . . . . . . . . 128

7.6.1 Bell-Mouth Based Estimation . . . . . . . . . . . . . . . . 128

$7.6 .2 \quad$ Measurement Delta . . . . . . . . . . . . . . . 128

7.6.3 Constant Gain Extended Kalman Filters Constructed Using the Proposed Equation Selection Procedure . . . . . . . . . . . 132

7.7 Conclusion . . . . . . . . . . . . . . . . . . . . . 132

8 Investigation of Fault Diagnosis in the Startup and Shutdown Operating Modes 135

8.1 Background . . . . . . . . . . . . . . . . . . . . . . 135

8.2 Test Construction Procedure . . . . . . . . . . . . . . . 136

$8.2 .1 \quad$ Fault Modeling . . . . . . . . . . . . . . . 136

8.2.2 Diagnosis Model . . . . . . . . . . . . . . . . . . . . 137

8.2 .3 Test Quantity . . . . . . . . . . . . . . . . . . . . . . . . . . . . . . . . . . 137

8.3 Simulation Study . . . . . . . . . . . . . . . . . . . . . . . . . . . . . . . . . . . . .

8.3.1 Fault Free Sequence . . . . . . . . . . . . . . . . . . . . . 139

8.3.2 Component Faults - Leakage in the Compressor and Increased Friction in Mechanical Bearings . . . . . . . . . . . . . . . 139

8.4 Conclusion . . . . . . . . . . . . . . . . . . . . . . 143

9 Diagnosis and Fault Tolerant Supervision of Industrial Gas Turbines 145

9.1 Introduction . . . . . . . . . . . . . . . . . . . 146

9.1.1 Problem Statement . . . . . . . . . . . . . . . . . . . . 147

9.1.2 Outline and Contributions . . . . . . . . . . . . . . . . . . . 148

9.2 Gas Turbine Diagnosis Modeling . . . . . . . . . . . . . . . . . . . 148

9.2.1 Measurement Signals . . . . . . . . . . . . . . . . . . . . . . . . . . . 149

9.2.2 Health Parameters . . . . . . . . . . . . . . . . . . . 150

9.2.3 Sensor and Actuator Faults . . . . . . . . . . . . . . 151

9.2.4 Constraints on Performance Deviation . . . . . . . . . . . . 152

9.2.5 Differential Algebraic Equation Form . . . . . . . . . . . . . . . . . . . . 153

9.2 .6 State Space Form . . . . . . . . . . . . . . . . . . . . . . . . . . . . . . . . . . . . . . . . . . .

9.3 Test Quantity Design . . . . . . . . . . . . . . . . . . . . . . . . . . 154

9.3.1 Constant Gain Extended Kalman Filter . . . . . . . . . . . . . . . . 155

9.3 .2 CUSUM Filtering . . . . . . . . . . . . . . . . . . . . . 156

9.4 Fault Isolation Method . . . . . . . . . . . . . . . . . . . . . . . . . . . 157

9.4.1 No Fault Hypothesis $H^{0} \ldots \ldots \ldots$. . . . . . . . . . . . . . . . . . . . . . . . . . . . . . . .

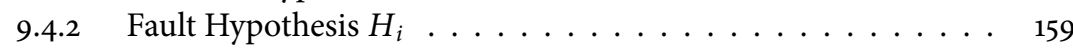

9.4.3 Component Failure and Foreign Object Damage . . . . . . . 159

9.5 Simulation Results . . . . . . . . . . . . . . . . . . . . . . . . . . . . . . . . . . . . 160

$9.5 .1 \quad$ Simulation Setup . . . . . . . . . . . . . . . . . . 160

9.5 .2 Fault Modes . . . . . . . . . . . . . . . . . . 162

9.5 .3 Fault Free Mode: $F_{N F} \ldots \ldots \ldots \ldots 162$

9.5.4 Sensor Fault Modes: $F_{p_{3}}, F_{t_{3}}$, and $F_{t_{7}} \ldots \ldots \ldots \ldots$

9.5.5 Actuator Fault Modes: $F_{m_{f}}$ and $F_{P_{A}} \ldots \ldots \ldots \ldots$ 
9.5.6 Sensor Fault Mode: $F_{n_{C 1}} \ldots \ldots \ldots \ldots \ldots \ldots$

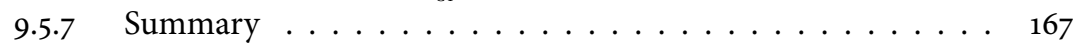

9.6 Experimental Case Studies . . . . . . . . . . . . . . . . . . . . . . . . 167

9.6.1 Evaluation 1: Fault Detection and Isolation . . . . . . . . . . . 168

9.6.2 Evaluation 2: Supervision Based on Fault Compensation in Test

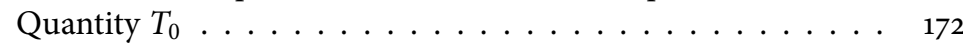

9.6.3 Evaluation 3: Fault Tolerant Supervision of Performance . . . . 174

9.6 .4 Summary . . . . . . . . . . . . . . . . . . . . . 175

9.7 Conclusion . . . . . . . . . . . . . . . . . . . . . 175

$\begin{array}{ll}10 \text { Conclusion } & 177\end{array}$

$\begin{array}{lr}\text { References } & 179\end{array}$

A Mole/Mass Conversions 187

A.1 Mole/Mass Fraction Calculation . . . . . . . . . . . . . . . . . . . . 187

A.2 Stoichiometry Matrix Expressed in Mass . . . . . . . . . . . . . . . . 188

A.3 Determination of Stoichiometric Air/Fuel Ratio . . . . . . . . . . . . . 189

B Measurement Plots 191

B.1 Ambient Temperature $T_{0} \ldots \ldots \ldots \ldots$. . . . . . . . . . . . . . . . . . . . . . . 192

B.2 Ambient pressure $p_{0} \ldots \ldots \ldots \ldots \ldots \ldots \ldots \ldots$

B.3 Shaft Speed $n_{C 1}$ of the Gas Generator . . . . . . . . . . . . . . . . . . . . 194

B.4 Generated Power $P_{A}$ by the Application . . . . . . . . . . . . . 195 

Part I

\section{Introduction}





\section{Chapter 1}

\section{Background and Motivation}

Diagnosis and supervision of industrial gas turbines are of vital importance since it gives valuable information about: (i) process health, (ii) instrumentation failure, and (iii) component faults. Air leakage in valves and bearing failures are examples of undesired behaviors which can be represented by the general term: component faults. When the actual health state of the gas turbine is known, it is easier to achieve: (i) more reliable operations, (ii) lower heat stress in components, (iii) lower fuel consumption, and (iv) more efficiently planed overhaul and maintenance. All these factors reduce the environmental impact and improving the operation profitability for the customer. The health state of an industrial gas turbine degrades gradually due to certain factors such as: (i) environment air pollution, (ii) fuel content, and (iii) ageing to mention some of the degradation factors. The compressor in the gas turbine is especially vulnerable against contaminants in the air since these particles are stuck at the rotor and stator surface. The loss in compressor performance, due to fouling, can partially be restored by an online or offline compressor wash. Sensor and actuator faults which are left undetected affect the engine's operation point and complicate the determination of a suitable time when the compressor should be washed. Thus, it is crucial to have a Fault Detection and Isolation (FDI) system to detect and isolate those faults at an early stage.

The deterioration in components affect the diagnosis statements and estimates used by the controller. For monitoring and control issues a good idea is to introduce parameters which represent correction factors of performance from nominal baseline. These parameters are denoted health parameters in the gas turbine diagnosis literature (Volponi, 2014). The motives to use those extra parameters are: (i) the health parameters are indicators for overhaul and maintenance, and (ii) the health parameters compensate for the deterioration in components which result in more reliable estimates of unmeasured signals used by, e.g., the controller.

In gas turbine diagnosis and control field, it is crucial to have good estimation of performance. Generally speaking, a model that has high accuracy gives smaller prediction errors than a model with a lower accuracy. The same argument is also valid 
for model-based diagnosis statements. Thus, one valuable factor which increase the diagnosis performance, i.e., increase the fault detection probability and lower the false alarm probability, is the usage of a model with high accuracy within the diagnosis tests. A common approach is to have diagnosis tests (or filters) which are based on a physical engine model (Kobayashi et al., 2005), thus if any components in the model are removed or replaced, also the filters need to be redesigned. The design of those filters might be a complex task and involves a lot of manual work, especially when the model is a large nonlinear differential algebraic equation (DAE) model. Thus, a systematic and automatic design procedure of the filters is desirable when the FDI-system is constructed.

Another perspective is the ability to simplify the FDI-system design by using available models, which are already developed, in the diagnosis tests. The industry partner Siemens Industrial Turbomachinery AB (SIT) in Finspång has provided a reference gas turbine model which is developed and evaluated during a long period of time. The reference model is built from an in-house thermodynamic library SITLib which is implemented in the modeling language Modelica. The reference model together with its surrounding components is mainly used for performance analyses and other in-house tools are considered for diagnosis and supervision statements. An overall idea with this work is to integrate the performance model also in the design of the FDI-system. A lot of work and money have been spent on development, validation, and maintenance of the reference model. Thus, a good idea to reuse as much knowledge as possible from the model used for performance analysis also in the FDI-system which is simplified by using a structured and systematic approach when the diagnosis tests are constructed. With the tool chain from the physical model to the performance monitoring that is proposed and demonstrated in the thesis such a benefit can be achieved.

\subsection{Problem Statement}

The objective of this work is to investigate a model based approach for diagnosis and supervision of industrial gas turbines. Since the available gas turbine fleet consists of a large number of individuals, where all of them have their own properties and are running under different ambient conditions, it is desirable that the design of the diagnosis and supervision system is systematic. The intention with a systematic design is: (i) the diagnosis tests for different gas turbine hardware configurations should be generated easily, and (ii) the equations which are consider in the diagnosis tests should be selected carefully from the model used for performance analysis. The systematic design is especially important since the available reference gas turbine model, used for performance analysis, is a large differential algebraic equation (DAE) model which is nonlinear. Early investigations show that the reference model has unobservable state variables which need to be removed when observer based diagnosis tests are constructed. 


\subsection{Thesis Outline}

The thesis is divided into two main parts. The first part addresses the gas turbine modeling and the systematic design of the FDI-system. In the second part, the FDI-system is evaluated for different configuration using case studies with simulated and experimental data.

The first part consists of Chapters 4, 5, and 6. Chapter 4 summarizes usable thermodynamic concepts for gas turbine modeling. In Chapter 5 , the developed gas turbine modeling package is presented. Chapter 6 discusses how the diagnosis tests in the FDIsystem are constructed.

The second part consists of Chapters 7, 8, and 9. In Chapter 7, the FDI-system is evaluated where the focus is on estimation of performance when the engine degrades. Chapter 8 discusses how the methodology can be used to detect and isolate faults when the gas turbine startup and shutdown. In Chapter 9, the FDI-system is extended with more tests to also diagnose faults in sensor and actuator at the same time the performance is supervised. 



\section{Chapter 2}

\section{Industrial Gas Turbine Background and Simulation Environment}

\subsection{Gas Turbine Theory}

A gas turbine engine is preferably used in many application when mechanical power is desired since it has: (i) a high power-to-weight ratio, (ii) a robust design (few moving parts, one directional rotation, acceptable vibration, etc.), (iii) often high reliability, (iv) low lubricating oil consumption, (v) an exhaust gas where most of the waste heat is collected (which can be used in a combined cycle), (vi) low emissions of carbon monoxide (CO) due to the excess of oxygen, and (vii) a wide spectrum of usable fuels to mention some of the main advantages. Depending on the application, the mechanical power can also be converted to other energy forms, e.g., electricity in a generator. Another working area for gas turbines is the so-called mechanical drive where the applied load is, e.g., a pump or an external compressor.

To create mechanical power in the gas turbine, the first step is to compress the working fluid using the compressor. In the second step, fuel is burned in the compressed fluid supplied by the compressor and the temperature is increased. In the final step, the fluid is expanded through the turbine at the same time as the temperature and pressure are decreased. In the gas turbine, the working fluid is in most cases atmospheric air. The work that is left after the compression work is subtracted from the work generated by the turbine is the produced mechanical power which is transferred to the application.

\subsubsection{Brayton Cycle}

The open gas turbine cycle is best described by the Brayton cycle see, e.g., Giampaolo (2009); Horlock (2007); Saravanamuttoo et al. (2001). In the ideal Brayton cycle (expressed in temperature $T$ and entropy $s$ ) the entropy is constant during the compression (1-2) and the expansion (3-4) phases. A Brayton cycle with two turbines is shown in 


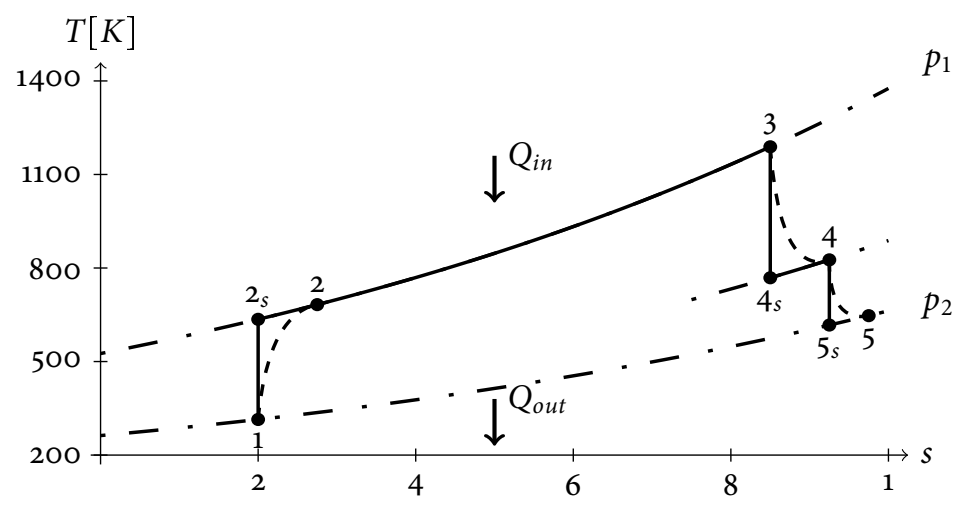

Figure 2.1: An ideal (solid lines), and a non ideal (dotted lines) Brayton cycle of a 2-shafted gas turbine is shown in the figure. In the non ideal cycle, the entropy in the compression and the expansion phase is not constant. This means that more work needs to be supplied in the compression phase and less heat is converted to work in the expansion phase, i.e., the entropy increases. In the non ideal gas turbine cycle, no pressure losses in components are considered. The numbers in the figure represent the gas path positions which are shown in Figure 2.4.

Figure 2.1. In the figure, the non ideal gas turbine cycle (dotted lines) is also shown where the entropy increases during the compression and the expansion phases. This leads to the fact that more work has to be supplied in the compression phase and less heat is converted to work in the expansion phase. The increase in entropy results in a lower efficiency of the engine. During the combustion process (2-3), the pressure is constant and the amount of heat $Q_{i n}$ is supplied by the fuel. Since the gas turbine is an open system, the original state is not reached which is required for a thermodynamic cycle. Ideally, the pressure in the inlet and outlet of the engine is the same. Thus, it can be assumed that the amount of heat $Q_{\text {out }}$ is left the engine and the cycle can be closed.

\subsubsection{Mechanical Drive Application}

Gas turbines used for mechanical drive applications are often smaller than engines used for power generation and have a typical power range of 1-50 MW. These engines are often featured with a twin shaft design (2-shaft) which means that the power turbine is free to move independently of the gas generator. The gas generator is used to produce hot gases which are delivered to the power turbine. The gas generator consists of a compressor and a turbine, which is also called a spool. Thus, a common configuration of an engine used for mechanical drive is denoted 1-spool and 2-shafted gas turbine. Since a mechanical connection between the gas generator and the power turbine is absent, it is possible to transform between rotational speed and delivered torque for a given amount of output power. Thus, this type of gas turbine is suitable for mechanical drive applications and at these sites (often in the connection with the oil and gas industry), the driven component is, e.g., a pump or an external compressor. The driven component can be used to pump gas in a pipeline where the speed is adjustable. 
An important parameter to maintain high efficiency is to have a high flame temperature in the combustor cans. The hot gases which left the combustor lead to an increased turbine inlet temperature (TIT). The turbine inlet temperature is often too high for the material in the first turbine blades which may results in undesired heat stresses. To reduce the heat stress in the turbines, compressed air is injected as a thin film at the surface of the blades to protect the material. In modern gas turbines, about $20 \%$ of the inlet air flow is bled off to perform cooling.

In Figure 2.2, the SGT-750 (successor to SGT-700) developed and manufactured by SIT in Finspång is shown. The typical application for the SGT-750 (SGT-700) 1-spool and 2-shafted gas turbine is mechanical drive. The inlet guide vanes (IGVs) are used to change the angle of the inlet mass flow to obtain as high efficiency as possible regardless of the load.

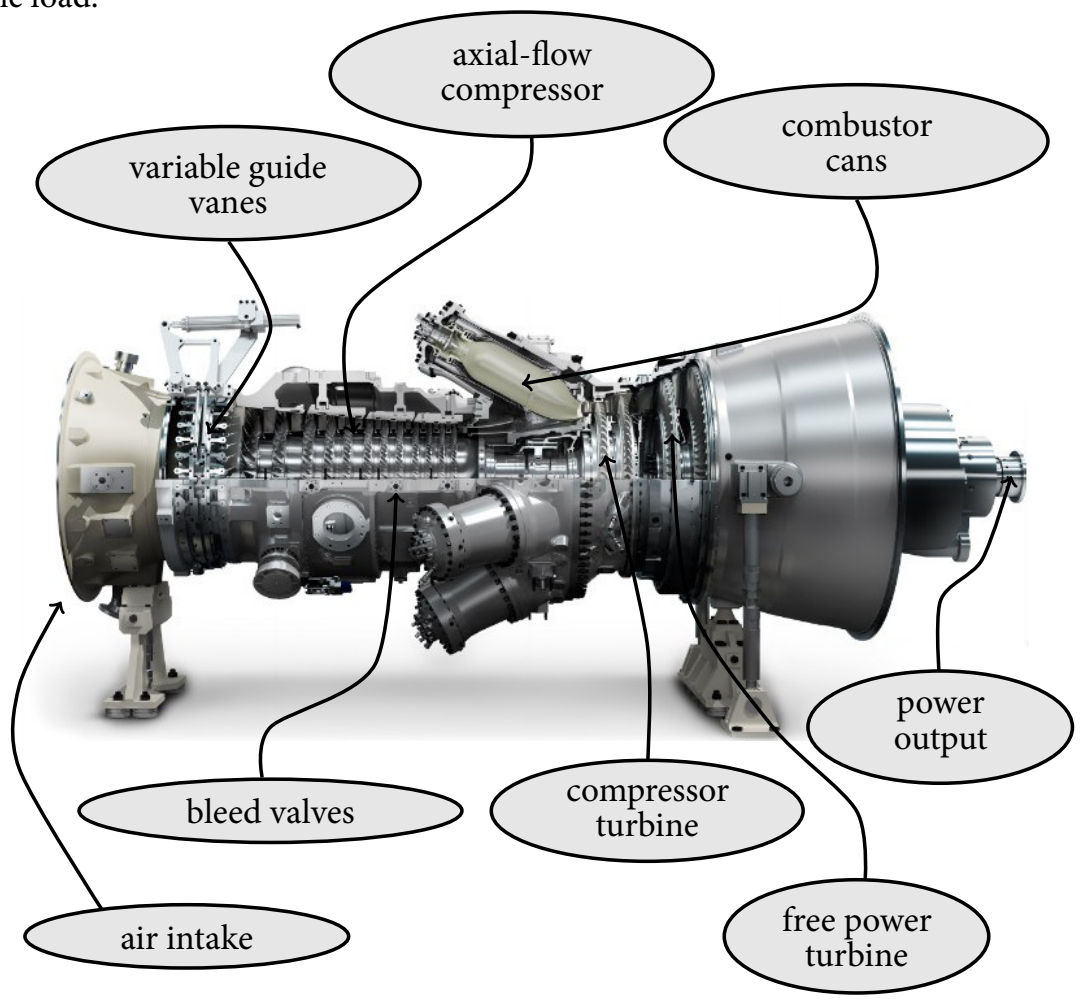

Figure 2.2: Typical appearance of a 1-spool and 2-shafted gas turbine used for mechanical drive applications. The engine shown in the figure is the SGT-750 (launched 2010 and successor to SGT-70o) developed and manufactured by SIT in Finspång. (Published with permission from Siemens AG, http://www. siemens. com/press)

The engine studied in the thesis is a 1-spool and 2-shafted gas turbine. For this type of engine, a gas generator which consists of a compressor $\mathrm{C} 1$ and a compressor-turbine $\mathrm{T} 1$ is connected with a power turbine T0. The gas generator is used to produce hot gases which are delivered to the power turbine. The work that is delivered to the application is taken from the power turbine. 


\subsection{SGT-70o Gas Turbine}

For the case studies and model development performed in the thesis, the work is applied to the SGT-70o gas turbine. The SGT-70o is chosen since models and experimental data that are received from SIT are for this gas turbine type. The developed methodology is general and is not just applied for the SGT-70o gas turbine. The SGT-700 is the predecessor to the SGT-750 shown in Figure 2.2 and is a 1-spool and 2-shafted engine used for mechanical drive applications. In Figure 2.3, the nominal performance chart of the SGT-70o gas turbine for different inlet temperatures and power turbine speeds is shown. According to the figure, the operating range is large. For the ambient temperature $15^{\circ} \mathrm{C}$ the speed of the power turbine and nominal shaft power can be varied between 3500-7000 rpm and 22-32 MW, where the efficiency increases with the power turbine speed. The power used for the actual application can be lower than the nominal shaft power shown in the figure.

\subsubsection{Measurement Signals}

A schematic representation of the SGT-700 gas turbine is shown in Figure 2.4 together with a common set of measurement sensors. The sensors measure temperatures $T_{i}$ pressures $p_{i}$, and shaft speeds $n_{i}$ throughout the gas path. The sensor position for the measured quantity of temperature or pressure is located at different cross-sectional areas $i$ along the engine's gas path. The measurement setup for the SGT-70o gas turbine is: (i) temperature $T_{2}$ and pressure $p_{2}$ before the compressor, (ii) discharge temperature $T_{3}$ and pressure $p_{3}$ after the compressor, (iii) temperature $T_{7}$ and pressure $p_{8}$ after the powerturbine, (iv) speed of gas generator $n_{C 1}$ and the power-turbine $n_{T 0}$, and (v) temperature $T_{0}$, pressure $p_{0}$, and relative humidity $\varphi_{0}$ of the ambient air. The subscript notation $i$ in the measured quantity describes at which cross-sectional area the sensor is located. A low number represents the air entrance and a high index number represents the position where the exhaust gas leaves the gas turbine. The sensor position of the gas turbine is sketched in Figure 2.4. The other measured signals in the figure are: (vi) the mass flow of fuel $m_{f}$, (vii) the generated power $P_{A}$, and (viii) the rotational speed $n_{A}$ of the driven component. The power signal is estimated by the application and not directly measured.

In some of the cross-sectional areas, the quantity is measured with more than one sensor. For example, the discharge pressure $p_{3}$ is measured with the three sensors $p_{3,1}$, $p_{3,2}$, and $p_{3,3}$. The exhaust temperature $T_{7}$ is measured with sensors in three rings where each ring has 16 thermocouples. The total number of sensors that measure the temperature $T_{7}$, at different location around the circumference of the three rings, is 48. The large number of thermocouples in the exhaust gas is used, e.g., to monitor the burners in the combustion chamber to discover if any burner has, e.g., a poor flame.

\subsubsection{Actuator Signals}

Input signals to the gas turbine are the valve positions. The actuators adjust the positions of the compressor bleed valves and combustor bypass valve. The bleed valves are usually used during startup and shutdown sequences to avoid stall and surge in the compressor. 


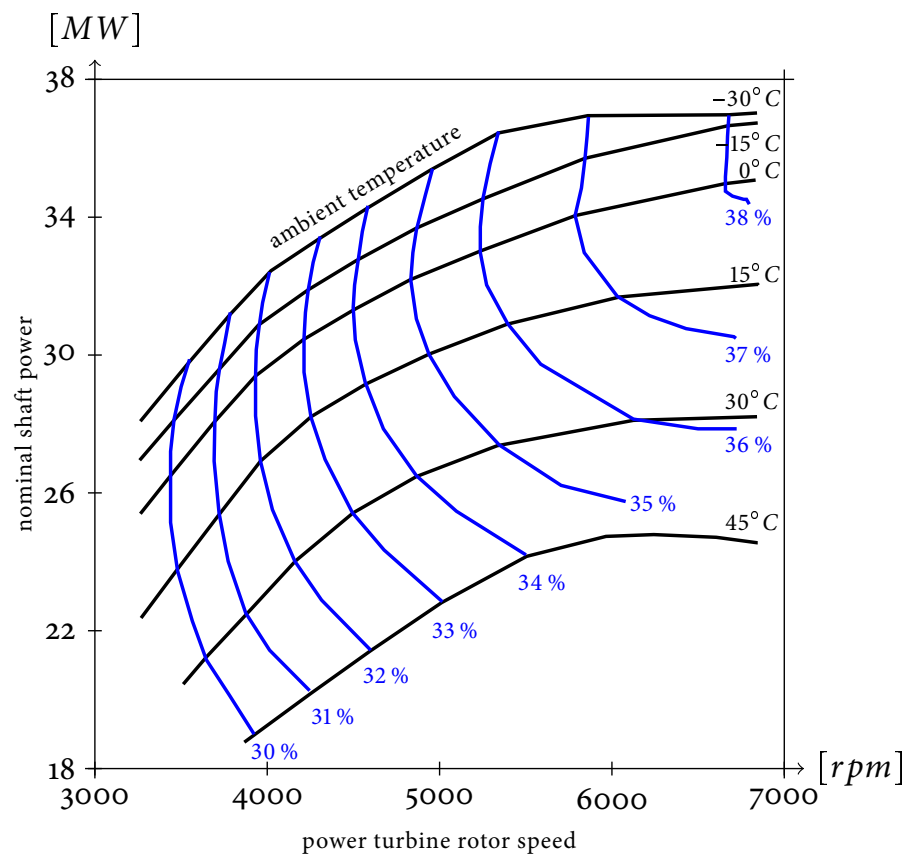

Figure 2.3: Nominal performance chart of the SGT-70o gas turbine for different inlet temperatures and power turbine speeds when a mechanical drive application is considered. The performance is described using the nominal shaft power and efficiency. (Source: SGT-7oo Industrial Gas Turbine Brochure, http://www .energy . siemens.com) 
At full load operations the bleed valves are fully closed. The combustor bypass valve is used to control the emission and stability when the load is reduced.

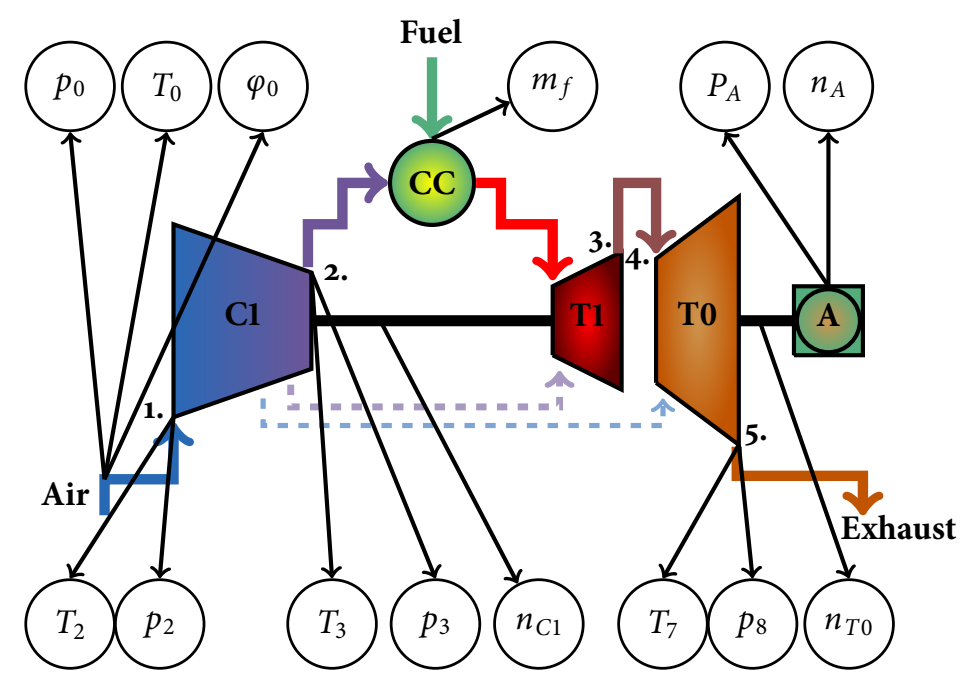

Figure 2.4: An overview of the 1-spool and 2-shafted SGT-70o gas turbine is shown in the figure. This gas turbine consists of a gas generator (compressor Cl and a compressor-turbine T1), a power turbine $\mathrm{T} 0$, and an external application $\mathrm{A}$. The gas generator supplies the power turbine with hot gases and the power turbine delivers the work demanded by the application. The cooling air, tapped from the compressor, is shown with dashed arrows in the figure.

\subsection{Simulation Platform}

For the work in the thesis, a simulation platform is provided by SIT. The simulation platform includes the reference gas turbine which is built from an in-house thermodynamic library SITLib. The provided simulation platform consists of: (i) a controller, (ii) a fuel system, (iii) a starter motor, (iv) a transmission, and (v) a 2-shafted gas turbine (reference model). The simulation platform and its components are shown in Figure 2.5. All of these components are written in the modeling language Modelica and are simulated using the tool Dynamic Modeling Laboratory (Dymola). The simulation platform can be used to investigate: (i) different fuel setups, (ii) startup and shutdown effects, (iii) variation in speed and power of applied load, and (iv) change in ambient environment conditions to mention some of the simulation scenarios. The ambient conditions can be changed according to: (i) the pressure $p_{0}$, (ii) the temperature $T_{0}$, and (iii) the relative humidity $\varphi_{0}$ of the incoming air. A variation of these ambient conditions can affect, e.g., the efficiencies and mass flows along the engine's gas path. The change in ambient conditions has a direct impact on the efficiency and the mass flow since, e.g., the pressure ratio over the compressor is affected. The change in ambient conditions also modifies the mixture of substances in the ambient air, i.e., the amount of water steam in the air. This effect 


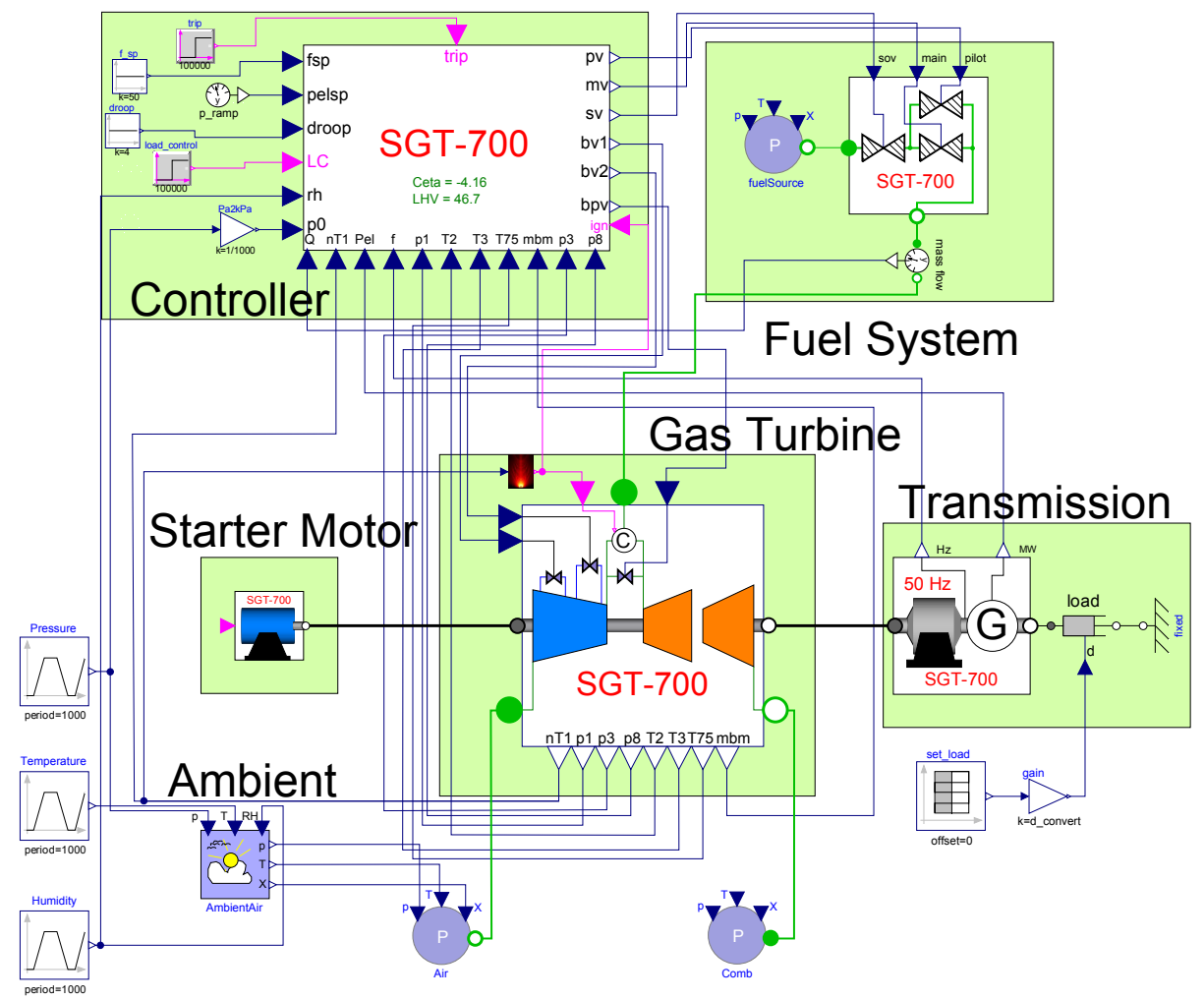

Figure 2.5: The simulation platform used for, e.g., performance analysis consists of a controller, a fuel system, a starter motor, a transmission, and a 2-shafted gas turbine. All of these components are implemented in the Modelica language and are simulated using the tool Dymola. The input signals are the ambient pressure $p_{0}$, the ambient temperature $T_{0}$, the relative humidity $\varphi_{0}$ of ambient air, and the demand power and frequency of the applied load. 
may also affect the performance parameters along the engine's gas path.

The advantage with the simulation platform is the ability to study reliably performance evaluations and parameter estimations throughout the engine's gas path due to different operational conditions. The input signals to the simulation platform are, e.g.,: (i) the properties of the ambient air, and (ii) the demand power and frequency of applied load. In Figure 2.5, the simulation platform is shown where the application is a $50 \mathrm{~Hz}$ electrical generator. When a mechanical drive application is evaluated, the electrical generator is replaced with a load where the speed is adjustable.

\subsubsection{Reference Gas Turbine Model}

A major part of a gas turbine model is the description of the gas media along the engine's gas path. The gas media is used to specify the thermodynamic properties of the fluid. To simplify the handling of the thermodynamic properties in Modelica, they can be encapsulated by a gas model. In the reference gas turbine model (developed by SIT), the Modelica Media package is utilizes which is a part of the standard Modelica package. The gas model in Modelica Media is flexible since substance mixtures of ideal gases with an arbitrary concentration can be modeled. This approach gives a number of equations in the gas turbine model that increases drastically with the number of substances in the gas. The number of equations is increased since each substance in the gas is described by a separate state variable in each control volume in the model. This result in, roughly speaking, that the number of substances in the gas is proportional to the number of equations in the overall gas turbine model. The reference model has about 2500 equations and 6o state variables which are considered large. More details about the reference gas turbine model, in an early stage, can be found in Idebrant and Näs (2003).

\subsubsection{Modelica}

Modelica is an equation based object oriented modeling language with focus on reusing components and model libraries. In an equation based language the relationships between variables are specified by the user simultaneously as the causality is left open. An open causality means that the order to calculate the variables does not have to be specified by the user. An example is a fluid which obey the ideal law. When the control volume model is designed, the user specifies the relation of the involved variables according to ideal law, i.e, the relationship $p v=R T$ in the model component. In this simple example, the pressure $p$, the specific volume $v$, and temperature $T$ can be calculated depending on the available input signals or the surrounding variables. This, together with the object oriented nature of the language simplifies the construction of component libraries since models can be reused where the same base class model can be used in all the three control volume cases.

Another advantage with the Modelica language is the concept of multi-domain modeling which means that different kinds of physical domains can be encapsulated in the same model. In the available simulation platform, shown in Figure 2.5, the considered domains are: (i) the thermodynamic, (ii) the mechanical, and (iii) the electrical domain. Between domains and components, information is only exchanged through special 
connection points. Those connection points are called connectors in Modelica. There are basically two kinds of quantities in a connector and these quantities are either defined as a flow, or a non-flow quantities. In a connection point, flow variables are summed to zero and non-flow variables are set equal.

In Modelica, state equations and algebraic constraints can be mixed which results in a model that is in a differential algebraic equation (DAE) form. For a differential algebraic equation model, the DAE-index of the model is an important property. For simulation purposes, a state-space form of the system model is desirable and the DAE-index is one measure of how easy or hard it is to obtain a state-space form. In general, higher index problems are often more complicated than lower index problems to simulate. Simulations of DAE systems are well described in Hairer et al. (1991); Ascher and Petzold (1998).

See the language specification at the webpage in Modelica Association (2007), or the textbooks by Fritzson (2004); Tiller (2001) for a comprehensive description of the Modelica language. In Casella et al. (2006), the Media library available in the standard Modelica package is presented. The Media library is utilized in the reference gas turbine model developed by SIT. 



\section{Chapter 3}

\section{Summary of Contributions}

The central topics in this work are the systematic design and the evaluation of a Fault Detection and Isolation (FDI) system for an industrial gas turbine. For the FDI-system design procedure, a thermodynamic modeling library GTLib is developed which can be used to build up a physical model of an industrial gas turbine. This model is then used for performance analysis and as a starting point when the diagnosis test quantities which are implemented as Constant Gain Extended Kalman Filters (CGEKFs) are designed. The designed CGEKFs have been given different diagnosis properties and together they are used in an FDI-system to calculate the actual health state of the engine and possible diagnoses. The systematic design procedure assures that relevant equations are chosen when the test quantities are generated.

The constructed FDI-system is evaluated using case studies based on simulated and experimental data. It is shown that the designed filters can be helpful when decisions about a fouled compressor are taken. When the FDI-system consists of more than one filter, sensor and actuator faults can be diagnosed at the same time as the performance is supervised. Slow varying sensor and actuator bias faults are difficult to diagnose (since they can appear in a similar manner as the performance deterioration), but the FDI-system has the ability to detect these faults. With the proposed method, component faults can be diagnosed during engine startup and shutdown sequences. Since loads and stresses in components increase during startup and shutdown, it can be a good idea to study component failure during these sequences.

\subsection{Thesis Summary}

The thesis is divided into two main parts, where the first part consists of the modeling work of the gas turbine and the FDI-design. In the second part, the FDI-system is evaluated for different configuration using case studies with simulated and experimental data. 
In Chapter 4, Thermodynamic Concepts, basic thermodynamic concepts that are used to describe the gas are presented. The gas model is a part of the GTLib package and is used along the engine's gas path. In the chapter, the combustion of air and fuel is introduced which is based on the assumption of a stoichiometric combustion. The state of the gas in a control volume is specified using the three state variables: pressure $p$, temperature $T$, and air/fuel ratio $\lambda$. When the state variable $\lambda$ is known, the mass fraction of species in the exhaust gas can be calculated. Here, the gas with substances: argon $(\mathrm{Ar})$, oxygen $\left(\mathrm{O}_{2}\right)$, nitrogen $\left(\mathrm{N}_{2}\right)$, carbon dioxide $\left(\mathrm{CO}_{2}\right)$, and water $\left(\mathrm{H}_{2} \mathrm{O}\right)$ are considered. With the air/fuel ratio description, pure atmospheric air can be described with an infinitely large air/fuel ratio $\lambda$.

In Chapter 5, GTLib - Thermodynamic Gas Turbine Modeling Package, the focus is on the implementation of the gas turbine components described in Chapter 4. These components are then used in an introductory control volume example where the objective is on variation in ambient conditions. The constructed (in GTLib) gas turbine model used for performance analysis is also shown in this chapter.

In Chapter 6, Modeling, Analysis, and Transformation of the Diagnosis Model, the objective is to design the diagnosis model, and transform this model to a form suitable for simulation. In the diagnosis model, a number of extra estimation parameters, i.e., the health parameters are introduced. These parameters should capture deviation in performance due to fouling, and other factors that can affect the performance. In this step, also the sensor and actuator fault modes can be specified together with the measurement setup. The equations, which are used in each diagnosis test, are then selected by structural methods. Since observer based tests are derived in Chapter 7 , the state variables in the derived test equations must be observable. An observability analysis, together with an index reduction are performed to get the test equations. A number of parsers is presented in the chapter. These parsers are used to transform the diagnosis model into runnable Matlab code. The Matlab environment is used here because of the available tools for diagnosis analysis that are implemented in Matlab.

In Chapter 7, Performance Estimation in Industrial Gas Turbine Engines, three studies are presented where techniques of performance deterioration estimations are investigated. In the first study, a simple approach to calculate deterioration due to compressor fouling is presented. In the next two studies, the gas turbine model is used as a base for the estimation techniques. In the second study, the estimations are based on so-called measurement deltas, which are generally the difference between the simulated, and the measured gas path quantity. In the third study, a nonlinear Kalman observer is evaluated on two test cases. In the first test case, simulated data from the reference platform is evaluated for different operational points and different atmospheric weather conditions. In the second test case, experimental data from a gas turbine mechanical drive site in the Middle East is evaluated.

In Chapter 8, Investigation of Fault Diagnosis in the Startup and Shutdown Operating Modes, a pilot study is presented where the objective is to investigate and develop a diagnosis test quantity used for detection and isolation of component faults in the startup and shutdown sequences of the gas turbine application. Since the engine goes through many operating modes for this run, the potential of the nonlinear Kalman filter 
used for fault estimation and supervision can be utilized fully.

In Chapter 9, Diagnosis and Fault Tolerant Supervision of Industrial Gas Turbines, the objective is to diagnose faults in sensor and actuator signals at the same time the performance is supervised. To increase the fault detectability, limitations of the health parameters are introduced. This limitation is introduced since the health parameters, for some fault modes, may capture the fault which results in residuals which can not be discriminated from the non-faulty case since measurement noise is present in the signals.

\subsection{Contributions}

Figure 3.1 gives an outline of the main methodological contributions of the thesis and the dashed line shows the boundaries between this work and material provided by SIT. Analysis of the gas turbine model provided by SIT shows that it is unnecessary complex for diagnosis purposes, especially with respect to the gas medium. Thus, the first step (a) is to replace the SITLib with the GTLib. The backward arrows symbolize that the model constructed using GTLib can be used together with the provided simulation platform. The GTLib based model and the SITLib based model (reference model) give the same outputs when: (i) air, fuel, and exhaust gases are used as the working fluids, and (ii) ambient conditions are not changed. In step (b), the diagnosis properties of the test quantity are specified. Since Modelica is an object oriented language, model specifications from GTLib based model can be inherited. In the final step (c), the test quantity is automatically generated using developed parsers. The overall benefit with the design procedure is to ensure that the accuracy of the diagnosis test quantity in step (c) is the same as in the model used for performance analysis in step (a).

The main contributions are summarized in:

- A thermodynamic modeling library GTLib implemented in Modelica is developed. Using GTLib, a gas turbine model can be constructed which and used for performance analysis and diagnosis modeling. In GTLib, the gas medium behavior is more efficiently modeled but still accurately describe machine behavior. In the diagnosis model, parameters to estimate component deteriorations and faults are introduced. These extensions are necessary to be able to design a model based diagnosis system.

- Based on the diagnosis models, which are of considerable size and complexity, a second key contribution is an automated FDI-system design method. The method, described in Section 6, aims to keep the required human work to a minimum. Important is also that the design technique is not gas turbine specific, but is applicable to any Modelica based model.

- The third contribution is the case study in Section 7 where the automated design procedure is applied to a gas turbine diagnosis model and experimentally validated using measured data from an industrial gas turbine site. 


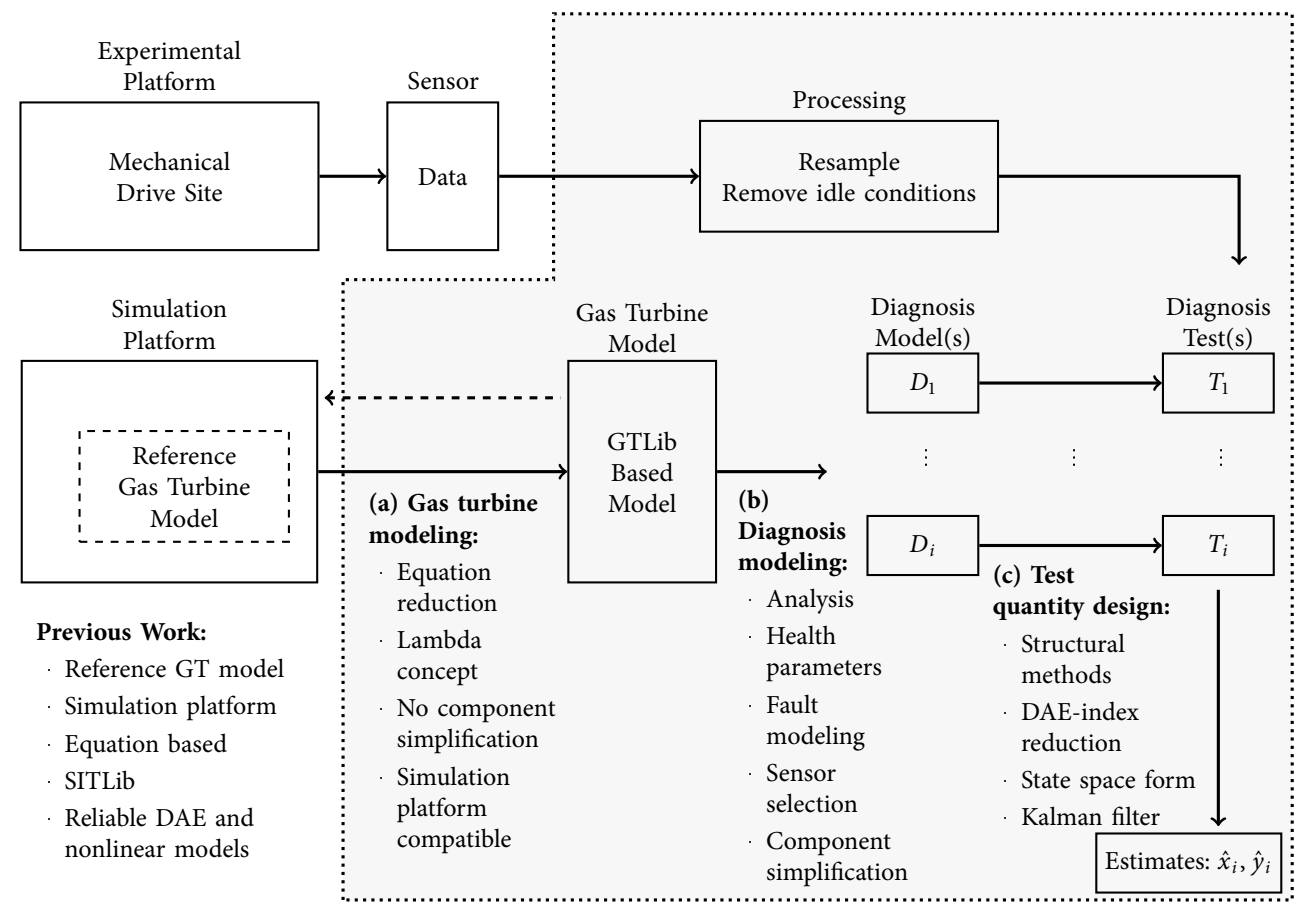

Figure 3.1: An overview of the experimental and simulation platform together with the key contribution of the systematic test quantity design procedure is shown. The FDI-system design consists of the three main steps: (a) gas turbine modeling, (b) diagnosis modeling, and (c) test quantity generation. The goal with the design is to have the same performance and accuracy in the FDI-system as in the gas turbine model used in the simulation platform simultaneously as the design procedure is simple enough. 
- The forth contribution is a fault tolerant FDI-system which is presented in Section 9. The proposed FDI-system can be used to detect and isolate a single sensor or actuator fault at the same time the performance is supervised.

\subsection{Publications}

The thesis is based on the work presented in the following publications.

\section{Journal Papers}

- Emil Larsson, Jan Åslund, Erik Frisk, and Lars Eriksson. Gas turbine modeling for diagnosis and control. Journal of Engineering for Gas Turbines and Power, 136 (7):17 pages, July 2014a. doi:10.1115/1.4026598

\section{Submitted}

- Emil Larsson, Jan Åslund, Erik Frisk, and Lars Eriksson. Diagnosis and fault tolerant supervision of industrial gas turbines. Submitted for journal publication, $2014 \mathrm{~b}$

\section{Conference Papers}

- Emil Larsson, Jan Åslund, Erik Frisk, and Lars Eriksson. Fault isolation for an industrial gas turbine with a model-based diagnosis approach. ASME Conference Proceedings, 2010(43987):89-98, 2010. doi:10.1115/GT2010-22511

- Emil Larsson, Jan Åslund, Erik Frisk, and Lars Eriksson. Health Monitoring in an Industrial Gas Turbine Application by Using Model Based Diagnosis Techniques. ASME Conference Proceedings, 2011(54631):487-495, 2011. doi:10.1115/GT201146825

- Emil Larsson, Jan Åslund, Erik Frisk, and Lars Eriksson. Fault Tolerant Supervision of an Industrial Gas Turbine. ASME Conference Proceedings, 2013(55188), 2013. doi:10.1115/GT2013-95727

\section{Licentiate Thesis}

- Emil Larsson. Diagnosis and Supervision of Industrial Gas Turbines. Technical report, Department of Electrical Engineering, 2012. LiU-TEK-LIC-2012:13, Thesis No. 1528, http://urn.kb.se/resolve?urn=urn:nbn:se:liu:diva-75985 



\section{Part II}

\section{Modeling and Design}





\section{Chapter 4}

\section{Thermodynamic Concepts}

The objective of this chapter is to present thermodynamic concepts that are useful in the development of a physical based gas turbine model used for: (i) performance analysis, (ii) supervision of components, and (iii) diagnosis statements. A central part in the gas turbine model is the description of the gas. During the combustion, fuel molecules react and break up into smaller molecules simultaneously as heat is released. Since different gas concentrations appear before and after the combustion, also the thermodynamic properties (e.g., enthalpy and heat capacity) of the fluid are changed. To reduce heat stresses in the first blades of the turbine, compressed fresh air is injected as a thin film at the surface of the blades to protect the material. These two factors result in a gas model which should be able to handle: (i) ambient air, (ii) exhaust gas, and (iii) a mixing between the two gases when a stoichiometric combustion using a hydrocarbon fuel is considered, i.e., when all hydrocarbons are completely oxidized to the products of carbon dioxide and water. Each fluid is described by a number of substances. The concentration of, e.g., the substances in the exhaust gas depends on the ambient conditions, the hydrocarbon fuel, and the air/fuel ratio. In Modelica standard library, thermodynamic properties such as heat capacity, enthalpy, and entropy are tabulated using the well known NASA polynomials (McBride et al., 2002) for a large number of species. Since these polynomials are provided by the standard library it is a good idea to incorporate this knowledge into the gas model.

\section{Outline of the Chapter}

The main focus in the chapter is to present the thermodynamic concepts which are used to describe the fluid throughout the gas path of the engine. In Section 4.1, a summary of basic thermodynamic relations (e.g., thermodynamic laws) and quantities (e.g, internal energy, and heat capacity) are presented which are used in the development of the gas model (Section 4.4). In Section 4.2, the description of the thermodynamic quantities such as: enthalpy, heat capacity, and entropy is introduced. These quantities are modeled using polynomial interpolation. Two types of combustion processes are presented in Section 4.3 
which are based on: (i) a chemical equilibrium calculation, and (ii) a stoichiometry combustion process. A comparison study between these two combustion models for different temperatures and air/fuel ratios is performed. The concept of a stoichiometric combustion is then incorporated in the gas model using the air/fuel ratio. The benefit with using the air/fuel ratio as a state variable is the reduction of model equations in the gas turbine model, which gives a model which is less computationally demanding and easier to handle in practise. In Section 4.4, the gas model is presented which is used in all thermodynamic components. In Section 4.5, the energy conservation for a mixture of ideal gases is derived, which leads to the specification of the state equations in the control volume model presented in Section 4.6.

\subsection{Thermodynamic System}

The intention with the present chapter is to introduce important relationships and give an introductory insight for models that are utilized when a thermodynamic system is designed. A thermodynamic system is defined as an amount of space together with a surrounding boundary against its environment where relationships between heat and work are studied. The thermodynamic system can either be open or closed. In an open system, the boundary lets mass, heat, and work passing through. In a closed system, the boundary only allows heat and work to be transferred. A gas turbine can be defined as a thermodynamic system since it converts heat (which is released from the fuel) to mechanical work (which is used to drive, e.g, a generator). Since a gas turbine exchange masses across the boundaries it is an open system, thus only open systems are studied in the thesis. An important thermodynamic component is a control volume. The control volume is central since it stores energy and keeps track of the state of the thermodynamic part of system. For a more comprehensive thermodynamic survey, see, e.g., Eastop and McConkey (1993); Heywood (1988); Borman and Ragland (1998); Turns (2006); Öberg (2009).

\subsubsection{Thermodynamic Quantities}

The state of a thermodynamic system can be described by a number of quantities. The most commonly occurring quantities are: temperature $T$, pressure $p$, specific volume $v$, mass $m$, enthalpy $h$, and and internal energy $u$. For a gas that occupy a volume $V$, the state of the gas can be described with an independent pair of thermodynamic quantities. Depending on this choice, the appearance of the described system equations are different. An example of an independent pair of state variables, for a known volume, is the pressure $p$ and temperature $T$. From the state variables, all the other thermodynamic quantities can be derived, e.g., mass $m$ and enthalpy $h$. In a thermodynamic system it is often possible to measure both the pressure and the temperature and therefore are these quantities called measured quantities since they are measurable. When a thermodynamic system is analyzed, it can be convenient to introduce quantities that are not directly measurable. These type of variables are called intermediate quantities. Internal energy $u$ and enthalpy $h$ are examples of such quantities. The relationship between internal energy 
$u$ and enthalpy $h$ is defined:

$$
h=u+p v
$$

where $p$ is the pressure, and $v$ is the specific volume of the gas. In open systems, the enthalpy is suitable to consider since it encapsulates both the internal energy and the work applied to the system. The applied work comes from the flowing fluid which affects the control volume.

\section{Intensive and Extensive Properties}

Thermodynamic quantities which do not vary with the size of the system is classified as an intensive property. Temperature $T$, pressure $p$, and density $\rho$ are examples of intensive properties. Quantities which do vary with the size of the system has an extensive property. Volume $V$, and total energy $U$ are examples of extensive properties. For each extensive property, an intensive property can be obtained when the quantity is divided by, e.g., the mass. Mass specific quantities are denoted with lower case letters in the thesis. Upper case letters are usually applied to denote the total amount of a certain quantity in the system. In some cases it is more suitable to consider the mole specific quantities. In the thesis, the tilde convention over the corresponding mass specific quantity is used. The total energy can, e.g., be expressed either in mass or in mole according to:

$$
U=m u=n \tilde{u}
$$

where $m$ is the total mass, and $n$ is the total number of moles in the gas. Since the molar mass is defined $M=m / n$, the relationship between the internal energy in (4.2) is:

$$
u=\frac{\tilde{u}}{M}
$$

\section{Specific Heat Capacities}

To describe the amount of energy that is needed to increase the temperature of the fluid one degree, for a unit mass, the specific heat capacities are used. Since the amount of energy that is required for a system that undergoes a constant-volume or a constantpressure thermodynamic process is different, two specific heat capacities $c_{v}$ and $c_{p}$ are defined according to:

$$
c_{v}=\left(\frac{\partial q}{\partial T}\right)_{v}, c_{p}=\left(\frac{\partial q}{\partial T}\right)_{p}
$$

where $q$ is the supplied heat, $v$ denotes a constant-volume process, and $p$ denotes a constant-pressure process. An example of a constant-volume process is a fluid in a bomb calorie meter and an example of a fluid that undergoes a constant-pressure combustion process is the fluid in a bunsen burner. The combustion chamber in a gas turbine is typically described by a bunsen burner. A fluid which goes from an initial state to a final state is said to be reversible if the fluid can be restored to their original state at the same time no change in its surroundings is caused. The heat capacities of a reversible process 
can be written:

$$
c_{v}=\left(\frac{\partial u}{\partial T}\right)_{v}, c_{p}=\left(\frac{\partial h}{\partial T}\right)_{p}
$$

where the first law of thermodynamics (4.7) for a reversible thermodynamic process (4.8) together with the differential of the enthalpy definition (4.1) are considered. The ratio of the specific heat capacities $\gamma$ is defined:

$$
\gamma=\frac{c_{p}}{c_{v}}
$$

The specific heat ratio is frequently used when an isentropic compression process or an expansion process is considered.

\subsubsection{Thermodynamic Laws}

The first law of thermodynamics states that the energy in a system that undergoes a closed thermodynamic cycle cannot either be created, or destroyed. The energy is merely converted between thermal energy (heat) and mechanical energy (work). For a thermodynamic cycle that is open, the intrinsic energy of the fluid can increase or decrease. The first law of thermodynamics is written:

$$
d U=d Q+d W
$$

where $U$ is the internal energy, $Q$ is the supplied heat, and $W$ is the supplied work. The sign conventions of the energy flows are shown in Figure 4.1, where positive flow directions point into the control volume.

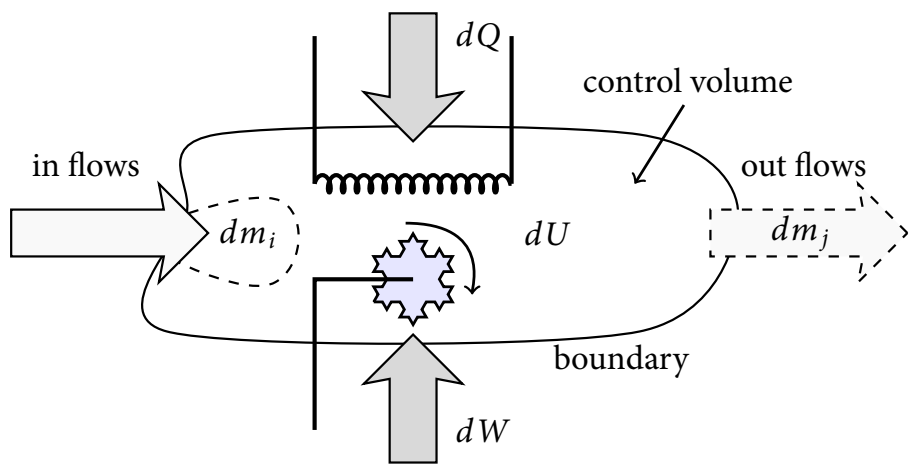

Figure 4.1: Sign conventions for an open thermodynamic system are shown in the figure. Positive flow directions point into the control volume. For a time interval $d t$, the amount of heat $d Q$ is added to the system, the work $d W$ is done on the system, the mass $d m_{i}$ is added to the system, and $d m_{j}$ is removed from the system.

When the system undergoes a reversible thermodynamic process, the supplied work is $d W=-p d V$ and the first law of thermodynamics is rewritten:

$$
d U=d Q-p d V
$$


where $p$ is the pressure and $V$ is the volume. The second law of thermodynamics is written:

$$
\frac{d Q}{T} \leq d S
$$

where $S$ is the entropy, and $T$ is the temperature. The equality in (4.9) holds for all reversible processes.

\subsection{Thermodynamic Properties of Pure Substances}

A gas media, used in a thermodynamic system, can either consist of a pure substance or a mixture of substances. These substances are called species. The atmosphere air media consists of the species, e.g., nitrogen, oxygen, and argon. Once the composition of the gas mixture is known, thermodynamic properties such as enthalpy, entropy, and heat capacity can be determined either on a mass basis, or on a mole basis as shown in:

$$
\begin{aligned}
h & =\sum x_{i} h_{i}, & \tilde{h} & =\sum \tilde{x}_{i} \tilde{h}_{i} \\
s & =\sum x_{i} s_{i}, & \tilde{s} & =\sum \tilde{x}_{i} \tilde{s}_{i} \\
c_{p} & =\sum x_{i} c_{p, i}, & \tilde{c}_{p} & =\sum \tilde{x}_{i} \tilde{c}_{p, i}
\end{aligned}
$$

where $x_{i}$ is the mass concentration, and $\tilde{x}_{i}$ is the mole concentration of substance $i$.

In this section, the thermodynamic properties for the species in (4.10) will be presented. To describe these thermodynamic properties of an ideal gas, tabulated data can be used. The NIST-JANAF thermochemical tables in Chase (1998) consist of tabulated data for a large number of species. The NIST-JANAF tables are well known, and the thermodynamic data is available in a wide range of pressures and temperatures with high accuracy. Since the data is in a tabular form, it can be necessary to interpolate between the points depending on the application.

Another method to describe gas properties is to use polynomial curve fitting techniques. The main advantage with using polynomials is the ability to encapsulate a large amount of thermodynamic data with only a few polynomial coefficients. Since polynomials are continuous, they can be differentiated easily and no interpolation is necessary, thus the simulation time can be reduced.

An early chemical equilibrium program contribution named $\mathrm{CEC}_{71}$ is presented in Gordon and McBride (1971). In CEC 71 , the heat capacity is described by a fourth-order polynomial with constant coefficients $a_{1}, \ldots, a_{5}$. These coefficients are approximated with a least-square technique (McBride and Gordon, 1992). To describe enthalpy and entropy the heat capacity coefficients are extended with the coefficients $a_{6}$ and $a_{7}$. For every species, two sets of coefficients are available. These sets are divided into a low temperature interval 200 to $1000 \mathrm{~K}$ and a high temperature interval 1000 to $6000 \mathrm{~K}$. The chemical equilibrium program (CEA) presented in Gordon and McBride (1994) is an extension of the previous developed CEC 71 program. In the new program, the thermodynamic heat capacity data is represented by two more coefficients. An additional temperature interval 6000 to $20000 \mathrm{~K}$ is also added for some of the substances. A summary of the NASA Glenn least-square coefficients and the tabulated thermodynamic 
data are shown in McBride et al. (2002). In the paper, the enthalpy of formation $\Delta_{f} h^{o}$ and the difference in enthalpy $\mathrm{HO}$ between the datum state temperature $T_{o}$ and temperature at $0 \mathrm{~K}$ are also tabulated.

\section{Datum State}

For thermodynamic systems which consist of a mixture of substances, the energy which is stored in chemical bonds must be included in the enthalpy description. Therefore, a datum state is defined for the temperature and the pressure. The reference state of the NASA Glenn polynomials (McBride et al., 2002) is represented by the datum temperature $T_{o}=298.15 \mathrm{~K}$, and the datum absolute pressure $p_{o}=1.01325 \mathrm{bar}=1 \mathrm{~atm}$. In the present section, the reference datum state is denoted with the super-script ${ }^{o}$. In other parts of the thesis, the datum state notation is omitted for simplicity.

\section{Reference Elements and Enthalpy of Formation $\Delta_{f} h^{o}$}

To each tabulated substance, a value called enthalpy of formation $\Delta_{f} h^{o}$ is assigned. The enthalpy of formation is defined to be the energy that is released when the substance is split up to its reference elements in the datum state. An example of reference elements are: argon $\mathrm{Ar}(\mathrm{g})$, carbon $\mathrm{C}(\mathrm{c})$, hydrogen $\mathrm{H}_{2}(\mathrm{~g})$, nitrogen $\mathrm{N}_{2}(\mathrm{~g})$, and oxygen $\mathrm{O}_{2}(\mathrm{~g})$. The symbol (g) indicates that the element is in a gaseous phase and the symbol (c) indicates that the element is in a condensed phase. For the reference elements the enthalpy of formation is equal to zero, hence:

$$
\Delta_{f} h^{o}\left(T_{o}\right)=0
$$

for the datum state temperature $T_{o}$.

\section{Standardized Enthalpy}

The standardized enthalpy $h^{\circ}(T)$, defined in Turns (2006), is the sum of the enthalpy of formation $\Delta_{f} h^{o}\left(T_{o}\right)$ and the sensible enthalpy $h_{s}^{o}(T)$. The enthalpy of formation takes into account the energy associated with the chemical bonds and the sensible enthalpy takes into account only the changes in temperature.

$$
h^{o}(T)=h^{o}\left(T_{o}\right)+\left[h^{o}(T)-h^{o}\left(T_{o}\right)\right]
$$

where the sensible enthalpy is defined:

$$
h_{s}^{o}(T) \equiv h^{o}(T)-h^{o}\left(T_{o}\right)=\int_{T_{o}}^{T} c_{p}(\tau) d \tau
$$

according to the definition (4.4) of the heat capacity $c_{p}$. For all substances at the datum state, the enthalpy of formation is arbitrary assigned the same value as the enthalpy:

$$
\Delta_{f} h^{o}\left(T_{o}\right) \equiv h^{o}\left(T_{o}\right)
$$

The standardized enthalpy (4.11) is now written:

$$
h^{o}(T)=\Delta_{f} h^{o}\left(T_{o}\right)+\int_{T_{o}}^{T} c_{p}(\tau) d \tau
$$


When another reference state is used, e.g., the reference state $T_{\hat{o}}=0 \mathrm{~K}$ it is possible to adjust (4.11) with the tabulated constant bias term $H 0$ :

$$
H 0=h^{o}\left(T_{o}\right)-h^{o}(0)
$$

since:

$$
h^{\hat{o}}(T) \equiv h^{o}(T)+H 0=\Delta_{f} h^{o}(298.15)+\int_{0}^{T} c_{p}(\tau) d \tau
$$

when the standardized enthalpy (4.14) is considered at the right hand side of (4.15).

\subsubsection{Specific Heat Capacity of Pure Substances}

The NASA polynomial for the specific heat capacity $\tilde{c}_{p}$ of a pure substance $i$ has the structure:

$$
\frac{\tilde{c}_{p, i}}{\tilde{R}}=a_{1, i} \frac{1}{T^{2}}+a_{2, i} \frac{1}{T}+a_{3, i}+a_{4, i} T+a_{5, i} T^{2}+a_{6, i} T^{3}+a_{7, i} T^{4}
$$

where the constants $a_{j, i}$ are the tabulated NASA Glenn Coefficients. The left hand side of (4.16) is a dimensionless quantity, hence it is possible to reformulate it as:

$$
\frac{\tilde{c}_{p, i}}{\tilde{R}}=\frac{c_{p, i}}{R}
$$

where $\tilde{R}$ is the universal gas constant, and $R$ is the specific gas constant. The relation between the gas constants is $\tilde{R}=M R$, where $M$ is the mole mass. Equation (4.17) shows that both the mass and the molar specific quantities can be calculated from the same polynomial coefficients.

\subsubsection{Standardized Enthalpy of Pure Substances}

The enthalpy is related thermodynamically to the heat capacity as follows:

$$
\frac{h(T)}{R T}=\frac{\int c_{p}(\tau) d \tau}{R T}+\frac{b_{1}}{T}
$$

where $b_{1}$ is an integration constant and the heat capacity $c_{p}$ is integrated with respect to the temperature $T$. To obtain the standardized enthalpy of the pure substances, the integration of (4.16) is performed to get:

$$
\begin{aligned}
\frac{h_{i}^{o}}{R T}=-a_{1, i} \frac{1}{T^{2}}+a_{2, i} \frac{\ln (T)}{T}+a_{3, i}+\frac{a_{4, i}}{2} & T+\frac{a_{5, i}}{3} T^{2}+ \\
& +\frac{a_{6, i}}{4} T^{3}+\frac{a_{7, i}}{5} T^{4}+b_{1, i} \frac{1}{T}
\end{aligned}
$$

where the constant $b_{1, i}$ is chosen to match (4.14) for the reference temperature $T=T_{o}$. Thus, the enthalpy of formation is included in the NASA polynomials by default. When the sensible enthalpy is needed, the enthalpy of formation is subtracted from the NASA polynomial calculations. The coefficients $a_{j, i}$ are the same as for the heat capacity in (4.16). 


\subsubsection{Standardized Entropy of Pure Substances}

The entropy is related thermodynamically to the heat capacity as follows:

$$
\frac{s(T)}{R}=\int \frac{c_{p}(\tau) d \tau}{R T}+b_{2}
$$

where $b_{2}$ is an integration constant. To obtain the standardized entropy of the pure substance $i$, the integration of $c_{p} / T$ in (4.16) is performed to get:

$$
\begin{aligned}
\frac{s_{i}^{o}}{R}=-\frac{a_{1, i}}{2} \frac{1}{T^{2}}-a_{2, i} \frac{1}{T}+a_{3, i} \ln (T)+a_{4, i} & +\frac{a_{5, i}}{2} T^{2}+ \\
& +\frac{a_{6, i}}{3} T^{3}+\frac{a_{7, i}}{4} T^{4}+b_{2, i}
\end{aligned}
$$

where $b_{2, i}$ is an integration constant. The coefficients $a_{j, i}$ are the same as for the heat capacity in (4.16).

\section{Entropy for an Ideal Gas}

For an ideal gas, the entropy depends on the temperature and the pressure. When the first (4.7) and second (4.9) law of thermodynamics are combined, together with the enthalpy definition (4.1) and an assumption of a reversible thermodynamic process (4.8), the entropy differential can be written:

$$
d s=\frac{c_{p}}{T} d T-\frac{R}{p} d p
$$

where the relation $d h=c_{p} d T$ of an ideal gas is introduced. To get an expression for the entropy, the differential (4.22) is integrated to get:

$$
s_{i}(T, p)=\int_{T^{o}}^{T} \frac{c_{p, i}(\tau)}{\tau} d \tau-R \ln \left(\frac{p}{p^{o}}\right)=s_{i}^{o}(T)-R_{i} \ln \left(\frac{p}{p^{o}}\right)
$$

where the entropy is calculated by the integration of $c_{p} / T$, i.e., the expression (4.21).

\subsubsection{Gibbs Free Energy}

When the temperature $T$ and the pressure $p$ are given, Gibbs free energy is feasible to consider in the determination of mixture concentration of substances which are in chemical equilibrium. Gibbs free energy is minimized when substances in a mixture are in chemical equilibrium. The definition of Gibbs free energy is:

$$
g(T, p)=h(T)-T s(T, p)
$$

where $h$ is the enthalpy, and $s$ is the entropy defined in (4.23). 


\subsection{Combustion}

For the modeling work, the combustion process is one of the central parts to consider in the gas turbine model. During the combustion, chemical energy in the fuel is released through dissipation of heat in the fluid simultaneously as the reactants break up into smaller substances. The composition of substances in the exhaust gas is different from the composition of the unburned mixture, which results in different thermodynamic properties of the gas before and after the combustion. With an assumption of a stoichiometric combustion, the concentration of the exhaust gas mixture is not dependent on the pressure and the heat which is released during the combustion. In a general case, the composition of the substances in the exhaust gas reacts with each other. The reacting rate depends on the temperature and the pressure of the fluid, i.e., so-called dissociation. Usually, a higher temperature and a higher pressure give a higher reacting rate. The number of dissociation products that have to be considered is also larger for higher temperatures and pressures. For example, nitrogen oxide $N O_{x}$ molecules appear in a lean exhaust gas for high temperatures. The dissociation products have a significant effect on the heat capacities, but a disadvantage with the consideration of a dissociation term in the gas model is the increasing complexity. For lower temperatures $(<1500 \mathrm{~K})$ a good simplification is to assume that the exhaust gas composition is frozen, i.e., independent of pressure and temperature so the dissociation terms can be neglected. This gives an assumption of a stoichiometric combustion.

A main goal here is to develop a gas model that can be used both for the compressed air and for the exhaust gas. This introduces the combustion into the gas model, where the concentration of the exhaust gas mixture is described using the air/fuel ratio $\lambda$ (see Section 4.3.1). The ambient air (or the air before the combustor) is described using a very large air/fuel ratio, i.e., $\lambda>>1$. In the gas model, dissociation effects are not described which result in an absence of $N O_{x}$ molecules. It will be shown that the dissociation effect for higher temperatures have a significant influence on the heat capacities (see Example 4.2). The advantages with the stoichiometric modeling approach are that the combustion can be described using only an extra state variable, the thermodynamic properties (e.g., enthalpy and heat capacity) vary with the air/fuel ratio, and it is simple to change the concentration of the reactants, i.e., the ambient air and the hydrocarbon fuel.

\section{Adiabatic Flame Temperature of a Constant-Pressure Combustion Process}

For an ideal gas turbine cycle, it is often assumed that the pressure during the combustion process is constant. For an adiabatic constant-pressure combustion process, it holds that the enthalpy $h_{u}$ before the combustion and the enthalpy $h_{b}$ after the combustion are the same. At least locally, $h_{b}$ is invertible which gives a solution for the flame temperature:

$$
T_{b}=h_{b}^{-1}\left(h_{u}\left(T_{u}\right)\right)
$$

where $T_{u}$ is the temperature of the unburned mixture, and $T_{b}$ are the temperature of the exhaust gas. Depending on the chosen pair of independent thermodynamic state variables the expression (4.25) needs to be evaluated or not evaluated in each control 
volume. When the temperature is chosen as a state variable, the enthalpy needs to be calculated in each control volumes and (4.25) has to be solved with a numerical solver. When the enthalpy is instead chosen as a state variable, it is not necessary to find an explicit solution of the temperature (and solve (4.25)) in each control volume. Solving (4.25) can be time consuming and can be avoided if the enthalpy is chosen as a state variable instead of temperature.

\subsubsection{Stoichiometry}

When sufficient oxygen is available in the air, a hydrocarbon fuel can be completely oxidized with the rest products of carbon dioxide and water. For the amount of air that just converts all hydrocarbons to carbon dioxide and water, a stoichiometric air/fuel ratio is defined. The stoichiometric air/fuel ratio can either be expressed in moles $(A / F)_{\tilde{s}}$ or in masses $(A / F)_{s}$. The actual air/fuel ratio $\lambda$, expressed in a mole basis of the mixture is defined:

$$
\lambda=\frac{n_{a} / n_{f}}{(A / F)_{\tilde{s}}}
$$

where $n_{a}$ is the mole number of air, and $n_{f}$ is the mole number of fuel. The air/fuel ratio, corresponding to an expression in mass basis, is defined:

$$
\lambda=\frac{m_{a} / m_{f}}{(A / F)_{s}}
$$

where $m_{a}$ is the mass of air, and $m_{f}$ is the mass of fuel. In general, stoichiometric air/fuel ratios expressed in mass and mole are not equal, i.e., $(A / F)_{\tilde{s}} \neq(A / F)_{s}$. A simple reaction formula for a hydrocarbon fuel, with emphasis on combustion, is presented in the following example.

Example 4.1

A hydrocarbon fuel propane $\mathrm{C}_{3} \mathrm{H}_{8}$ is combusted with air that consists of oxygen and nitrogen. A simple air model assumption is that for each oxygen molecule $\mathrm{O}_{2}, 3.773$ nitrogen molecules $\mathrm{N}_{2}$ are available. This gives the following reaction formula, in mole basis, for the combustion:

$$
\overbrace{\mathrm{C}_{3} \mathrm{H}_{8}}^{\text {Fuel }}+n \overbrace{\left(\mathrm{O}_{2}+3.773 \mathrm{~N}_{2}\right)}^{\text {Air }} \longrightarrow 3 \mathrm{CO}_{2}+4 \mathrm{H}_{2} \mathrm{O}+(n-5) \mathrm{O}_{2}+n 3.773 \mathrm{~N}_{2}
$$

where $n$ is the number of available oxygen molecules in the unburned mixture where one propane molecule is present. When $n=5$ the oxidization is complete, i.e., all hydrocarbons have been converted to carbon dioxide and water. If $n<5$, there are not enough hydrocarbons in the combustion so the formula has an excesses of oxygen. In the present example, it is assumed that $n \geq 5$, but for the case with a $n<5$, it is not enough oxygen molecules for the carbon dioxide molecule formations. Instead, the formula has to be extended with carbon oxide molecules $C O$ to preserve the number of atoms in the 
chemical reaction. Since the combustion in a gas turbine has an excesses of oxygen, this case is not considered in the gas model.

To simplify the presentation of the chemical reaction, it can be written in a matrix form according to:

$$
\overbrace{C_{3} H_{8}}^{\text {Fuel }}+n \overbrace{\left(\mathrm{O}_{2}+3.773 N_{2}\right)}^{\text {Air }} \longrightarrow(\underbrace{\left[\begin{array}{c}
0 \\
0 \\
n \\
3.773 n
\end{array}\right]}_{n_{\text {air }}}+\underbrace{\left[\begin{array}{c}
3 \\
4 \\
-5 \\
0
\end{array}\right]}_{\tilde{s}})^{T}\left[\begin{array}{c}
\mathrm{CO}_{2} \\
\mathrm{H}_{2} \mathrm{O} \\
\mathrm{O}_{2} \\
\mathrm{~N}_{2}
\end{array}\right]
$$

where the stoichiometric matrix $\tilde{S}$ consists of the coefficients of the hydrocarbon molecule combustion. The advantage with the introduced stoichiometric matrix is the ability to use fuels with several hydrocarbon molecules in a compact manner. The right hand side of (4.28) describes the number of constructed molecules in the combustion.

For $n=5$, the oxidation is complete and the stoichiometric air/fuel ratio can be calculated (i) in mole: $(A / F)_{\tilde{s}}=23.87$, and (ii) in mass: $(A / F)_{s}=15.68$ according to the definition of air/fuel ratio (4.26) and (4.27).

The chemical reaction formula in Example 4.1 can be extended to capture more general descriptions of the ambient air and the hydrocarbon fuel. Here, it is assumed that the fuel consists of the following molecules: methane $\mathrm{CH}_{4}$, ethane $\mathrm{C}_{2} \mathrm{H}_{6}$, propane $\mathrm{C}_{3} \mathrm{H}_{8}$, carbon dioxide $\mathrm{CO}_{2}$, and nitrogen $\mathrm{N}_{2}$. The chemical reaction for these species is written:

$$
\begin{aligned}
\mathrm{CH}_{4}+2 \mathrm{O}_{2} & \rightarrow \mathrm{CO}_{2}+2 \mathrm{H}_{2} \mathrm{O} \\
\mathrm{C}_{2} \mathrm{H}_{6}+3.5 \mathrm{O}_{2} & \rightarrow 2 \mathrm{CO}_{2}+3 \mathrm{H}_{2} \mathrm{O} \\
\mathrm{C}_{3} \mathrm{H}_{8}+5 \mathrm{O}_{2} & \rightarrow 3 \mathrm{CO}_{2}+4 \mathrm{H}_{2} \mathrm{O} \\
\mathrm{CO}_{2} & \rightarrow \mathrm{CO}_{2} \\
\mathrm{~N}_{2} & \rightarrow \mathrm{N}_{2}
\end{aligned}
$$

where the species of $\mathrm{CO}_{2}$ and $\mathrm{N}_{2}$ are unaffected by the combustion. The corresponding substances for the air are the following: argon $(\mathrm{Ar})$, carbon dioxide $\left(\mathrm{CO}_{2}\right)$, water $\left(\mathrm{H}_{2} \mathrm{O}\right)$, nitrogen $\left(\mathrm{N}_{2}\right)$, and oxygen $\left(\mathrm{O}_{2}\right)$. To get a more flexible description, also the concentrations of respective substance in the mixtures are considered. The mole concentration vectors of air $\tilde{x}_{a}$ and fuel $\tilde{x}_{f}$ are expressed according to:

$$
\tilde{x}_{a}=\left(\begin{array}{c}
\tilde{x}_{a, A r} \\
\tilde{x}_{a, C O_{2}} \\
\tilde{x}_{a, H_{2} O} \\
\tilde{x}_{a, N_{2}} \\
\tilde{x}_{a, O_{2}}
\end{array}\right), \tilde{x}_{f}=\left(\begin{array}{c}
\tilde{x}_{f, C H_{4}} \\
\tilde{x}_{f, C_{2} H_{6}} \\
\tilde{x}_{f, C_{3} H_{8}} \\
\tilde{x}_{f, C O_{2}} \\
\tilde{x}_{f, N_{2}}
\end{array}\right)
$$

where the sums of the elements are $\sum_{i} \tilde{x}_{a, i}=\sum_{i} \tilde{x}_{f, i}=1$. When the stoichiometric reaction formula in (4.29) is combined with the method described in Example 4.1, the 
following combustion formula can be written:

$$
\tilde{x}_{f}+n \tilde{x}_{a} \longrightarrow\left(n \tilde{x}_{a}+\tilde{S} \tilde{x}_{f}\right)^{T}\left[\begin{array}{c}
A r \\
C O_{2} \\
H_{2} O \\
N_{2} \\
O_{2}
\end{array}\right]
$$

where the stoichiometric matrix (Stephanopoulos et al., 1998):

$$
\tilde{S}=\left(\begin{array}{ccccc}
0 & 0 & 0 & 0 & 0 \\
1 & 2 & 3 & 1 & 0 \\
2 & 3 & 4 & 0 & 0 \\
0 & 0 & 0 & 0 & 1 \\
-2 & -3.5 & -5 & 0 & 0
\end{array}\right)
$$

is expressed in moles. The element $(i, j)$ in the matrix symbolize the number of air species $\tilde{x}_{a, i}$ that are created/depleted from each species $\tilde{x}_{f, j}$ in the fuel. In practice, it is more suitable to have the description in masses instead of moles. The conversion procedure is shown in Appendix A.2. The stoichiometric matrix can be rewritten:

$$
S=\left(\begin{array}{ccccc}
0 & 0 & 0 & 0 & 0 \\
\frac{M_{\mathrm{CO}_{2}}}{M_{\mathrm{CH}_{4}}} & 2 \frac{M_{\mathrm{CO}_{2}}}{M_{\mathrm{C}_{2} \mathrm{H}_{6}}} & 3 \frac{M_{\mathrm{CO}_{2}}}{M_{\mathrm{C}_{3} H_{8}}} & 1 & 0 \\
2 \frac{M_{H_{2} O}}{M_{\mathrm{CH}_{4}}} & 3 \frac{M_{\mathrm{H}_{2} O}}{M_{\mathrm{C}_{2} \mathrm{H}_{6}}} & 4 \frac{M_{H_{2} O}}{M_{\mathrm{C}_{3} H_{8}}} & 0 & 0 \\
0 & 0 & 0 & 0 & 1 \\
-2 \frac{M_{\mathrm{O}_{2}}}{M_{\mathrm{CH}}} & -3.5 \frac{M_{\mathrm{O}_{2}}}{M_{\mathrm{C}_{2} H_{6}}} & -5 \frac{M_{\mathrm{O}_{2}}}{M_{\mathrm{C}_{3} H_{8}}} & 0 & 0
\end{array}\right)
$$

where $M_{i}$ is the mole mass of substance $i$. The chemical reaction between the two gases air and fuel, can now be written:

$$
m_{a} x_{a}+m_{f} x_{f} \rightarrow m_{a} x_{a}+m_{f} S x_{f}
$$

where $m_{a}$ is the mass of air, and $m_{f}$ is the mass of fuel. The mass fraction of the burned gas arise when the right hand side of (4.34) is normalized. This gives an expression of the mass fraction of substances in the exhaust gas $x_{b}$ according to:

$$
x_{b}(\lambda)=\frac{m_{a} x_{a}+m_{f} S x_{f}}{m_{a} \sum_{i} x_{a, i}+\left.m_{f} \sum_{i} S x_{f}\right|_{\text {row }=i}}=\frac{(A / F)_{s} \lambda x_{a}+S x_{f}}{(A / F)_{s} \lambda+1}
$$

where $\sum x_{a, i}=1$, and $\left.\sum S x_{f}\right|_{\text {row }=i}=1$ because normalized mass flow concentrations are used and the number of atoms are conserved. To receive the final expression (4.35), the air/fuel ratio (4.27) is considered in the determination. The stoichiometric air/fuel ratio $(A / F)_{s}$ is calculated according to:

$$
(A / F)_{s}=\left(\frac{m_{a}}{m_{f}}\right)_{s}=\frac{2 \frac{x_{f, \mathrm{CH}_{4}}}{M_{\mathrm{CH}_{4}}}+3.5 \frac{x_{f, \mathrm{C}_{2} \mathrm{H}_{6}}}{M_{\mathrm{C}_{2} H_{6}}}+5 \frac{x_{f, \mathrm{C}_{3} \mathrm{H}_{8}}}{M_{\mathrm{C}_{3} H_{8}}}}{\frac{x_{a, O_{2}}}{M_{\mathrm{O}_{2}}}}
$$


where the concentration of oxygen in the exhaust gas in (4.34) is equal to zero and the calculations are shown in Appendix A.3. Equation (4.35) states that the mass fraction of the exhaust gas can be expressed only in the scalar variable $\lambda$ for the two gases air and fuel.

\subsubsection{Chemical Equilibrium}

For performance analysis, a good approximation is to assume that the substances produced by the combustion is in equilibrium. Equilibrium here means that the dissociation between the species occurs with equal rate, e.g., the number of $\mathrm{O}_{2}$ molecules dissociated into two $\mathrm{O}$ atoms is the same as the number of two $\mathrm{O}$ atoms that are dissociated into $\mathrm{O}_{2}$ molecules, i.e., $\mathrm{O}_{2} \leftrightharpoons 2 \mathrm{O}$.

This dissociation rate depends highly on the temperature, and increases with the increased temperature (Heywood, 1988). To calculate the chemical equilibrium for a specific exhaust gas at a given combustion temperature, a chemical equilibrium program can be used. A well known program is the NASA equilibrium program, presented in Gordon and McBride (1994). Here, the chemical equilibrium program CHEPP developed in Eriksson (2004) is utilized with some modification. In the original CHEPP version, hydrocarbons in the form: $\mathrm{C}_{a} \mathrm{H}_{b} \mathrm{OH}$, together with atmospheric air in the form: $\left(\mathrm{O}_{2}+\right.$ $\left.3.773 N_{2}\right)$ are considered as reactants. In the present work, the interface to CHEPP is modified to handle hydrocarbons, and atmospheric air in the form showed in (4.30). The modified CHEPP is used to check how well the thermodynamic properties of a stoichiometric chemical reaction in (4.34) harmonize with a gas in chemical equilibrium. In Figure 4.2, a demonstration of CHEPP's ability to calculate the mole concentration in the exhaust gas for an isooctane fuel $\mathrm{C}_{8} \mathrm{H}_{18}$ at three different temperatures is shown. As the figure indicates, the dominant species for $\lambda>1$ are: nitrogen $N_{2}$, carbon dioxide $\mathrm{CO}_{2}$, water $\mathrm{H}_{2} \mathrm{O}$, and oxygen molecule $\mathrm{O}_{2}$ when the temperature is low. For higher temperatures, the species of nitrogen oxide $N O$ increase in concentration. For $\lambda<1$, the shortage of oxygen molecules results in a reduction in carbon monoxide $C O$.

An introduction of how the equilibrium is calculated will follow in the remaining part of the section. For a more comprehensive explanation about equilibrium calculation see, e.g., Heywood (1988); Turns (2006); Öberg (2009). A commonly occurring method to calculate the chemical equilibrium is to minimize the thermodynamic potential of the mixture, e.g., the sum of Gibbs free energy in (4.24) for each substance in the mixture, i.e., $G(n)=\sum_{i} \tilde{g}_{i} n_{i}$. For the calculation, the temperature $T$ and pressure $p$ have to be specified. To find that $n$ which minimizes $G(n)$ an optimization procedure is performed together with a number of atom balance constraints. The optimization problem is written:

$$
\begin{array}{ll}
\underset{n}{\operatorname{minimize}} & G(n) \\
\text { subject to } & \sum_{i=1}^{l} \sum_{j=1}^{m} \tilde{a}_{i j} n_{j}=\tilde{b}_{i} \\
& n_{j} \geq 0
\end{array}
$$

where $l$ is the number of unique atoms, $m$ is the number of substances in the mixture 

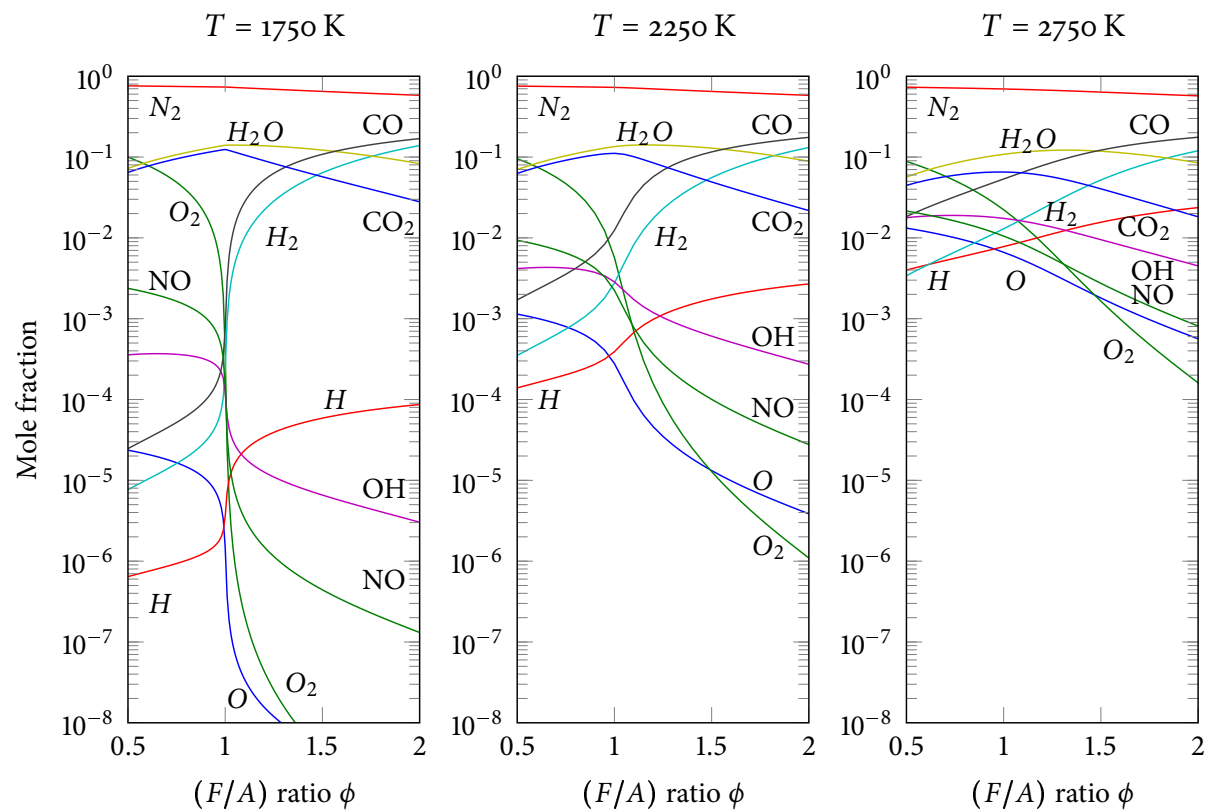

Figure 4.2: In the figure, the combustion product substances of an isooctane fuel $C_{8} H_{18}$ at chemical equilibrium for the temperatures $1750 \mathrm{~K}, 2250 \mathrm{~K}$, and $2750 \mathrm{~K}$ and pressure $1 \mathrm{~atm}$ is shown. The figure is generated in the chemical equilibrium program CHEPP, where the ten most affectable product species are considered. The species are plotted versus the fuel/air ratio $\phi$, where $\phi=\lambda^{-1}$. For lean combustions $(\phi<1)$ at temperature $1750 \mathrm{~K}$ the dominated species are: oxygen $\mathrm{O}_{2}$, carbon dioxide $\mathrm{CO}_{2}$, water $\mathrm{H}_{2} \mathrm{O}$, and nitrogen $\mathrm{N}_{2}$. For lean combustions at higher temperatures the nitrogen oxide $\mathrm{NO}$ and carbon monoxide $\mathrm{CO}$ substances increase in concentration. 
which is in equilibrium, and $G(n)=\sum_{i} \tilde{g}_{i} n_{i}$ is the cost function. The first step to set up the optimization problem is to select which product substances that should be used in the exhaust gas. This means that the structure of the concentration vector $\tilde{x}_{b}$ has to be specified. In the second step, the balance equations of the atom conservation are set up. In the conservation equations of atoms, $l$ different atoms and $m$ different substances in the exhaust gas is considered. In mole basis, these constraints are written $\tilde{A} n=\tilde{b}$. The $\tilde{A} \in \mathbb{R}^{l \times m}$ matrix describes which (and how many) atoms each combustion substance consists of. The solution to the optimization problem is the equilibrium gas composition, which is $\tilde{x}_{b}=\frac{n}{|n|}$ when the optimization is performed. In Example 4.2 the setup of the optimization problem is presented, and the results from the equilibrium calculation are shown in Figure 4.3.

Example 4.2

A hydrocarbon fuel with concentration $\tilde{x}_{f}$ is combusted with atmospheric air with concentration $\tilde{x}_{a}$. These two concentration vectors were introduced in (4.30). The exhaust gas concentration vector $\tilde{x}_{b}$ is calculated using the chemical equilibrium program CHEPP and is the solution to the optimization problem presented in (4.37). The product species in the exhaust gas are chosen to be: hydrogen $H$, nitrogen $N_{2}$, oxygen $O$, carbon monoxide $\mathrm{CO}$, carbon dioxide $\mathrm{CO}_{2}$, water $\mathrm{H}_{2} \mathrm{O}$, oxygen $\mathrm{O}_{2}$, nitrogen monoxide $\mathrm{NO}$, hydroxyl $\mathrm{OH}$, hydrogen $\mathrm{H}_{2}$, and argon $\mathrm{Ar}$. These substances are summarized in vector form:

$$
\tilde{x}_{b}=\left(\tilde{x}_{H}, \tilde{x}_{N_{2}}, \tilde{x}_{O}, \tilde{x}_{C O}, \tilde{x}_{\mathrm{CO}_{2}}, \tilde{x}_{\mathrm{H}_{2} \mathrm{O}}, \tilde{x}_{\mathrm{O}_{2}}, \tilde{x}_{\mathrm{NO}}, \tilde{x}_{\mathrm{OH}}, \tilde{x}_{\mathrm{H}_{2}}, \tilde{x}_{A r}\right)^{T}
$$

The chemical reaction formula between air and fuel can in this case be written:

$$
\begin{gathered}
\overbrace{n_{a}\left(\tilde{x}_{A r} A r+\tilde{x}_{\mathrm{CO}_{2}} \mathrm{CO}_{2}+\tilde{x}_{\left.\mathrm{O}_{2} \mathrm{O}_{2}+\ldots\right)}\right.}^{\text {Air }}+\overbrace{n_{f}\left(\tilde{x}_{\mathrm{CH}_{4}} \mathrm{CH}_{4}+\tilde{x}_{\left.\mathrm{C}_{2} \mathrm{H}_{6} C_{2} \mathrm{H}_{6}+\ldots\right)}\right.}^{\text {Fuel }} \\
\underbrace{n_{1} \mathrm{H}+n_{2} \mathrm{~N}_{2}+n_{3} \mathrm{O}+\ldots+n_{11} A r}_{\text {Exhaust Gas }}
\end{gathered}
$$

where $n_{a}$ is the number of mole air, $n_{f}$ is the number of mole fuel, and $n_{i}, i=1, \ldots, 11$ depends on the temperature and the pressure. To get the concentration vector $\tilde{x}_{b}, n$ is normalized. In this case, unique atoms in the reactants are: hydrogen $H$, nitrogen $N$, oxygen $O$, carbon $C$, and argon $A r$. Thus the coefficients $\tilde{a}_{i j}$ for the balance equations can be summarized in the matrix:

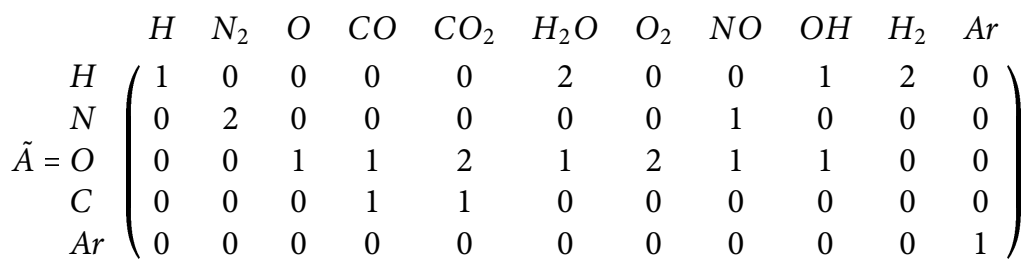


and the vector $\tilde{b}$ is constructed according to:

$$
\tilde{b}\left(\tilde{x}_{a}, \tilde{x}_{f}\right)=\left(n_{H}, n_{N}, n_{O}, n_{C}, n_{A r}\right)^{T}
$$

where the number of moles in $\tilde{b}$ depends on the amount of air and fuel. The relative measure between the atoms can be controlled by the $\lambda$ parameter. In Figure 4.3, the result of the CHEPP calculation (in mass concentration) is compared with a mass fraction calculation based on the stoichiometric combustion in (4.34) for the most dominant substances. The more general equilibrium calculation was shown in Figure 4.2 but without including argon $(A r)$ in the mixture.

\subsubsection{Comparison of the Heat Capacity Between the Stoichiometric Gas Description and the Chemical Equilibrium Calculation}

The most commonly occurring thermodynamic properties of a gas are, e.g., the enthalpy $h$, the entropy $s$, the internal energy $u$, the density $\rho$, the gas constant $R$, and the heat capacities $c_{p}, c_{v}$. An idea is to check how well these properties are described for different gas description approaches. Here, the behavior of the stoichiometric combustion (4.34) and the chemical equilibrium calculation (4.37) presented in Example 4.2 is investigated for the heat capacity $c_{p}$. In Figure 4.3, the mass concentrations of substances in the two modeling approaches are shown. The main difference between these two approaches is the increase in concentration of the nitrogen oxide molecules. The chemical equilibrium calculation is strongly connected to the increase in temperature which leads to a higher amount of nitrogen oxide molecules. The change in mass concentration of species affects the thermodynamic properties mentioned previously, and in Figure 4.4 the heat capacity is compared for the two approaches. In the figure, three different combustion temperatures: $T=1000 \mathrm{~K}, T=1500 \mathrm{~K}$, and $T=2000 \mathrm{~K}$ are compared for the two modeling approaches. The figure indicates that the mismatch between the two cases appears to increase for large temperatures and for temperatures above $2000 \mathrm{~K}$ the mismatch is larger than $8 \%$. For temperatures below $\approx 1500 \mathrm{~K}$, the stoichiometric description agrees with the chemical equilibrium calculation. In all three cases the results are as expected.

\subsubsection{Mixing of Exhaust Gases with Different Lambda}

It is important to have the potential to mix two (or more) gases with different air/fuel ratio. For example, a way to increase the efficiency is to increase the combustion temperature. To reduce the heat stresses which appear in the first blades of the turbine, compressed fresh air is injected as a thin film at the surface of the blades to protect the material. After some time, the injected air and the exhaust gas are mixed which changes the thermodynamic gas properties of the fluid. Therefore it is important to have a model library that can handle the mixture between exhaust gases with different air/fuel ratios and fresh cooling air. The mass concentration $x_{b}$ of the gas, that is a mixture of the two 


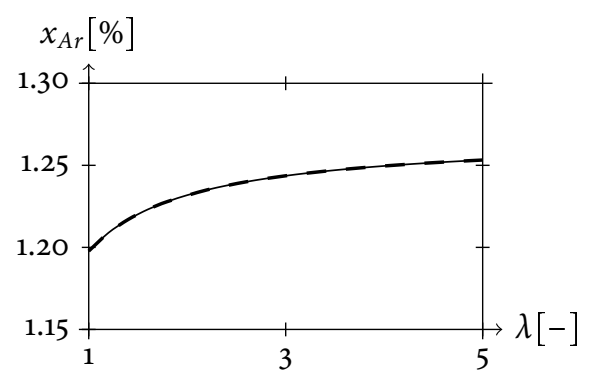

(a) $\mathrm{Ar}$

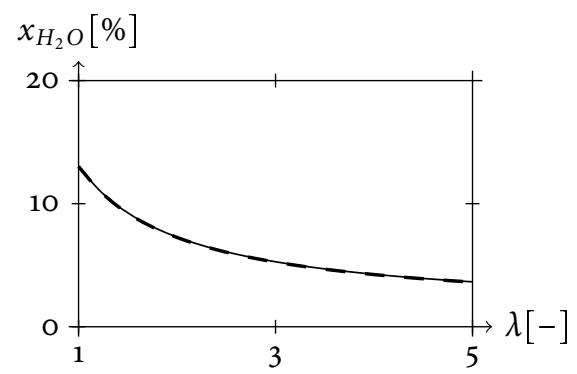

(c) $\mathrm{H}_{2} \mathrm{O}$

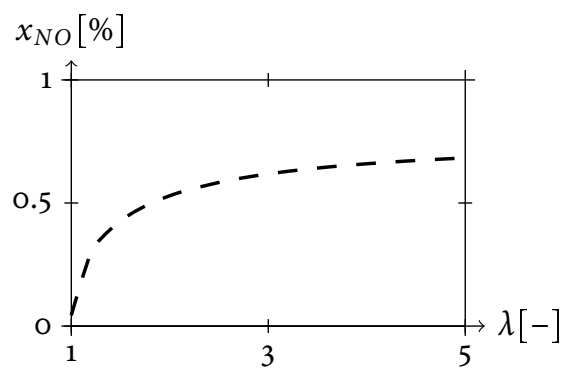

(e) $\mathrm{NO}$

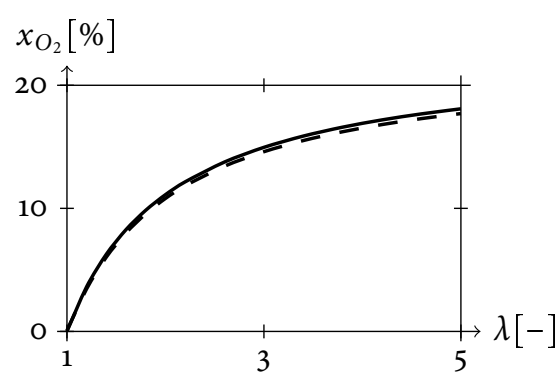

(b) $\mathrm{O}_{2}$

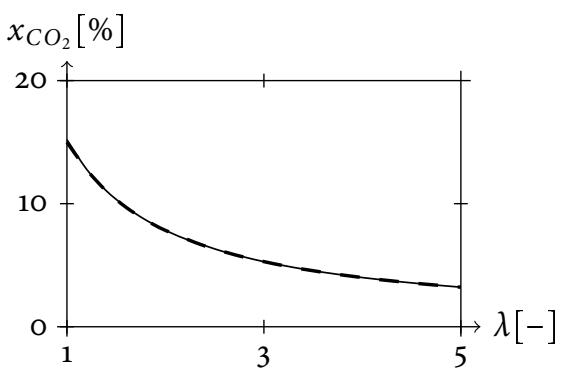

(d) $\mathrm{CO}_{2}$

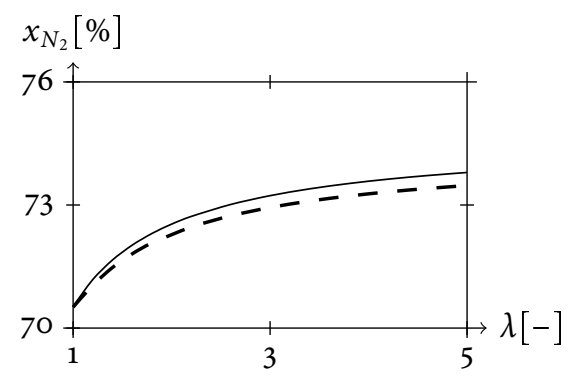

(f) $\mathrm{N}_{2}$

Figure 4.3: Mass fraction of the substances argon $(\mathrm{Ar})$, oxygen $\left(\mathrm{O}_{2}\right)$, water $\left(\mathrm{H}_{2} \mathrm{O}\right)$, carbon dioxide $\left(\mathrm{CO}_{2}\right)$, nitrogen oxide $(\mathrm{NO})$, and nitrogen $\left(\mathrm{N}_{2}\right)$ in the exhaust gas when the combustion is lean, the temperature is $2000 \mathrm{~K}$, and the pressure is $1 \mathrm{~atm}$. The dashed lines in each figure represent substances for equilibrium calculations performed in CHEPP, and described in Example 4.2. Solid lines represent calculations made according to stoichiometric combustion presented in (4.34). For these two cases, the same air $x_{a}$, and fuel $x_{f}$ mass concentration vectors are used. The nitrogen oxide $N O$ is not considered in the stoichiometric combustion, and is not shown here. The other substances that $\tilde{x}_{b}$ consists of, showed in (4.38) are not showed here since they are too small. The main difference between these two cases is the appearance, and the increase of nitrogen oxide in the CHEPP calculation. 


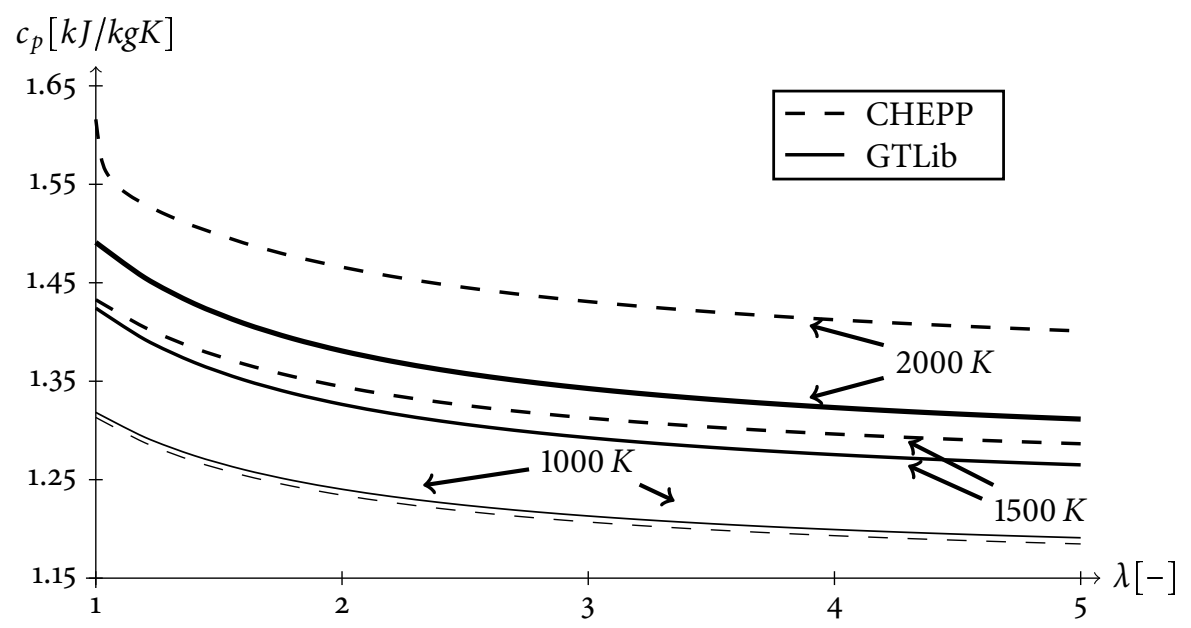

Figure 4.4: In the figure, the heat capacity $c_{p}$ is calculated for the two calculation methods: stoichiometric combustion, and chemical equilibrium calculation. For these two methods, the combustion is lean and three temperatures are studied. The temperatures are: $T=1000 \mathrm{~K}, T=1500 \mathrm{~K}$, and $T=2000 \mathrm{~K}$. As the figure indicates, the difference between these two cases appears to increase for high temperatures. For temperatures above $2000 \mathrm{~K}$, the mismatch between the two calculation methods is larger than $8 \%$. For temperatures below $\approx 1500 \mathrm{~K}$, the stoichiometric description agrees with the chemical equilibrium calculation.

fluids 1 and 2, is:

$$
x_{b}(\lambda)=\frac{m_{1} x_{b}\left(\lambda_{1}\right)+m_{2} x_{b}\left(\lambda_{2}\right)}{m_{1}+m_{2}}
$$

where $m_{i}$ is the mass of fluid $i$, and $\lambda_{i}$ is the air/fuel ratio of fluid $i$.

Solving (4.40) gives an analytic solution of the air/fuel ratio $\lambda$ in the mixed gas as:

$$
\lambda=\frac{\left(m_{1} \lambda_{1}+m_{2} \lambda_{2}\right)+\lambda_{1} \lambda_{2}(A / F)_{s}\left(m_{1}+m_{2}\right)}{\left(m_{1} \lambda_{2}+m_{2} \lambda_{1}\right)(A / F)_{s}+\left(m_{1}+m_{2}\right)}
$$

where the mass concentration vector in (4.35) is used in the determination. The expression (4.41) is used in the turbine component in the GTLib package where the cooling air is mixed with the exhaust gas. The masses in (4.41) can directly be translated to masses per unit time, i.e., mass flows.

\subsection{Ideal Gas Model}

The gas model is accessible in each thermodynamic component in the gas turbine package where the gas properties are calculated. The state variables: pressure $p$, temperature $T$, and air/fuel ratio $\lambda$ are considered as input variables to the gas model. The thermodynamic properties which are always calculated by the gas model are: the specific 
internal energy $u$, the density $\rho$, the specific enthalpy $h$, and the gas constant $R$. The gas model can be extended to also calculate, e.g., the heat capacity $c_{p}$, and the entropy $s$. To simplify the model representation, the gas model is divided into two parts: (i) an equation based part, and (ii) a function based part. The basic idea is that the equation based part consists of fundamental constraints and the function based part is used for calculations of thermodynamic properties, e.g., typically described by look-up tables. The same procedure is utilized in the original Modelica Media package. The structure of the gas model is shown in Figure 4.5 where the dotted arrows represent quantities which

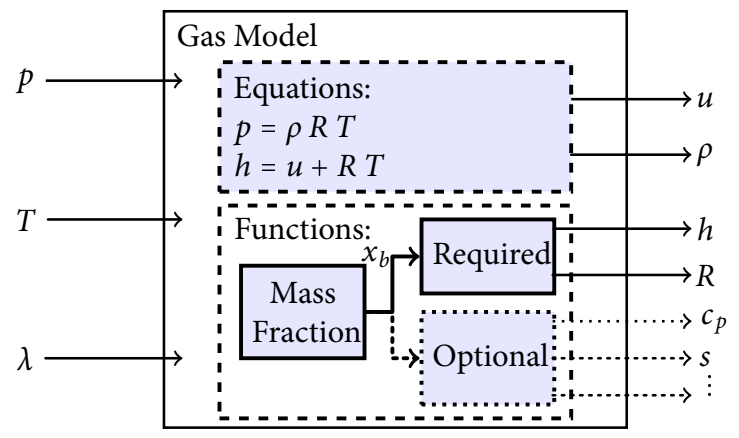

Figure 4.5: Structure of the gas model.

are not calculated by default. The equation based part relies on the ideal gas law (4.42a), and the relation between enthalpy and internal energy (4.1) of an ideal gas. The equation based part is:

$$
\begin{aligned}
& p=\frac{m}{V} R T=\rho R T \\
& h=u+p V=u+R T
\end{aligned}
$$

where $p$ is the pressure, $m$ is the mass, $V$ is the volume, $R$ is the gas constant, $T$ is the temperature, $\rho$ is the density, $u$ is the specific internal energy, and $h$ is the specific enthalpy.

Since the gas is described by a mixture of ideal gas substances, the mass fraction calculation must be encapsulated by the gas model. The mass fraction calculation is linked together with the thermodynamic properties of the gas and form the function based part. The thermodynamic property of each substance in the function based part relies on the NASA Glenn coefficients McBride et al. (2002). The mass fraction calculation is based on (4.35) where the mass fraction of air $x_{a}$, the mass fraction of fuel $x_{f}$, and the stoichiometric air/fuel ratio $(A / F)_{s}\left((A / F)_{s}\right.$ is a function of $x_{a}$ and $\left.x_{f}\right)$ are all assumed to be constant since the ambient conditions are not changed. Thus, those quantities are not input signals to the gas model and are treated as constant values. When the ambient conditions change, those constant quantities have to be updated. 


\subsubsection{Thermodynamics Properties of Frozen Mixtures}

In the gas model, a stoichiometric combustion is considered together with an assumption of a frozen mixture of ideal gases. For a frozen mixture, the gas composition is independent of the pressure and temperature, see, e.g., Heywood (1988). Thus, the temperature dependent part can be separated from the air/fuel ratio dependent part as shown in (4.43a)-(4.43d) using the mass fraction vector in (4.35). The thermodynamic properties calculated by the function based part in Figure 4.5 are:

$$
\begin{aligned}
h(T, \lambda) & =\sum_{i} h_{i}(T) x_{b, i}(\lambda) \\
R(\lambda) & =\sum_{i} R_{i} x_{b, i}(\lambda) \\
c_{p}(T, \lambda) & =\sum_{i} c_{p, i}(T) x_{b, i}(\lambda) \\
s^{o}(T, \lambda) & =\sum_{i} s_{i}^{o}(T) x_{b, i}(\lambda)
\end{aligned}
$$

and the entropy (4.23) for an ideal gas is written:

$$
\begin{aligned}
& s(T, p, \lambda)=\sum_{i} s_{i}\left(T, p_{i}\right) x_{b, i}(\lambda)= \\
&=\sum_{i} s_{i}^{o}(T) x_{b, i}(\lambda)-\sum_{i} R_{i} \ln \left(\frac{p_{i}}{p^{o}}\right) x_{b, i}(\lambda)= \\
& \quad=s^{o}(T, \lambda)-R(\lambda) \ln \left(\frac{p}{p^{o}}\right)-\sum_{i} R_{i}(\lambda) x_{b, i}(\lambda) \ln \left(x_{b, i}(\lambda)\right)
\end{aligned}
$$

where the partial pressure $p_{i}=p x_{b, i}$ is introduced according to Dalton's law. The index $i$ denotes the thermodynamic property for the pure substances. For an entropy change $\left(\Delta s=s_{2}-s_{1}\right)$ where the substances in the mixture do not change the last term in (4.44) can be removed.

\subsection{Energy Conservation of Thermodynamic Systems}

The first law of thermodynamics (4.7) states that energy cannot be created or destroyed. The energy can only be transformed between different states of the fluid. In open thermodynamic systems, the transformation is between thermal energy $d Q$, mechanical work $d W$, and intrinsic energy $d U$ of the fluid. The objective with this section is to derive a relation between differentials of the fluid using the state variables pressure $p$, temperature $T$, and air/fuel ratio $\lambda$.

\subsubsection{Thermodynamic Differentials $d U, d W$, and $d Q$}

The thermodynamic differentials $d U, d W$, and $d Q$ are summarized in this section. 


\section{Internal Energy Differential $d U$}

The internal energy of the gas before and after the mixing occurs can be denoted $U_{0}$ and $U_{\Delta}$ according to:

$$
\begin{aligned}
U_{0} & =m u\left(T_{0}, \lambda_{0}\right)+\Delta m_{i} u\left(T_{i}, \lambda_{i}\right) \\
U_{\Delta} & =\left(m+\Delta m_{i}\right) u\left(T_{\Delta}, \lambda_{\Delta}\right)
\end{aligned}
$$

where the mass $\Delta m_{i}$ is added, the subscript $\Delta$ denotes the mixing properties, and the subscript $i$ denotes the incoming fluid $i$. The difference between the two fluid states can be described by a Taylor series expansion at point $\left(p_{0}, T_{0}, \lambda_{0}\right)$. The Taylor series expansion of $U_{\Delta}$ is:

$$
U_{\Delta}=\left(m+\Delta m_{i}\right)\left(u\left(T_{0}, \lambda_{0}\right)+\frac{\partial u}{\partial T} \Delta T+\frac{\partial u}{\partial \lambda} \Delta \lambda+O\left(\Delta^{2}\right)\right)
$$

where $O\left(\Delta^{2}\right)$ captures all the second order, and higher terms. The change in internal energy $\Delta U=U_{\Delta}-U_{0}$ can now be written:

$$
\Delta U=m\left(\frac{\partial u}{\partial T} \Delta T+\frac{\partial u}{\partial \lambda} \Delta \lambda\right)+\left(u\left(T_{0}, \lambda_{0}\right)-u\left(T_{i}, \lambda_{i}\right)\right) \Delta m_{i}
$$

where second order terms and higher are removed. The definition of the differential $d U$, together with (4.47), gives:

$$
d U=\lim _{t \rightarrow 0}\left(\frac{\Delta U}{t}\right)=m\left(\frac{\partial u}{\partial T} d T+\frac{\partial u}{\partial \lambda} d \lambda\right)+\left(u\left(T_{0}, \lambda_{0}\right)-u\left(T_{i}, \lambda_{i}\right)\right) d m_{i}
$$

\section{Work Energy Differential dW}

The gas stream that is flowing into the control volume does work on the thermodynamic system, thus the differential $d W$ had to be split into the two work contributions:

$$
d W=d \tilde{W}+p v_{i} d m_{i}
$$

where $d \tilde{W}$ is the external mechanical work, and $p v_{i} d m_{i}$ is the work performed by the mass differential $d m_{i}$. When no external work is applied to the system the differential $d \tilde{W}=0$.

\section{Thermal Energy Differential dQ}

The thermal energy differential $d Q$ is assumed to be known and when the container is perfectly insulated the differential $d Q=0$.

\subsubsection{Energy of the Mixture of Frozen Ideal Gases}

The first law of thermodynamics (4.7) can be written together with (4.48) and (4.49):

$$
m\left(c_{v}(T, \lambda) d T+\frac{\partial u(T, \lambda)}{\partial \lambda} d \lambda\right)+u(T, \lambda) d m_{i}=d Q+d \tilde{W}+h\left(T_{i}, \lambda_{i}\right) d m_{i}
$$


where the enthalpy $h=u+p v$ for the incoming mass flow and the specific heat capacity $c_{v}=\frac{\partial u}{\partial T}$ are introduced. When open systems are studied, it is convenient to consider the enthalpy since it encapsulates both the internal energy and the mechanical work of the inflowing masses.

\subsection{Control Volume Model}

To describe the fluid state in a perfectly mixed container, the differentials that are derived from the ideal gas law (4.51), and the differentials that are derived from the energy conservation equation (4.50) is considered. To completely specify the gas properties, also the mass concentration vector is needed. An idea is to use a chemical equilibrium program that calculate the concentration of products for a given temperature and pressure. In the present work, it is assumed that the gas composition is frozen, thus the composition depends only on the air/fuel ratio.

\subsubsection{Differential Form of the Ideal Gas Law}

The differential of the ideal gas law $(4.42 a)$ is:

$$
V d p=R T \sum_{i} d m_{i}+m T d R+m R d T
$$

where it is assumed that the size of the control volume is constant, i.e., $d V=0$. All differentials, except $d R$ are either requested or available. According to the chain rule, the differential $d R$ is calculated as:

$$
d R\left(p, T, x_{b}\right)=\frac{\partial R}{\partial p} d p+\frac{\partial R}{\partial T} d T+\left(\nabla x_{b} R\right)^{T} d x_{b}
$$

where $\nabla x_{b}$ is the gradient of the mass concentration vector $x_{b}$. It is assumed that no reaction between molecules occurs in the container, i.e., the gas composition is frozen, thus the differential (4.52) is:

$$
d R=\left(\nabla x_{b} R\right)^{T} d x_{b}
$$

\section{Mass Concentration Differential $d x_{b}$}

Since fluids with different mass concentrations can be mixed, an expression of the mass concentration differential vector $d x_{b}$ is needed. A schematic view of the process, for $i$ fluids which are flowing into a control volume $V$, is shown in Figure 4.6. In the figure, $d x_{b_{i}}$ is the concentration vector, and $d m_{i}$ is the mass of the inflowing fluid $i$. This gives an expression of the mass conservation in the perfectly mixed control volume according to:

$$
d\left(m x_{b}\right)=\sum_{i} x_{b_{i}} d m_{i}
$$

where the left hand side describes the mass change of species in the control volume $V$. The mass change of the species in $V$ is equal to the sum of the incoming and outgoing 


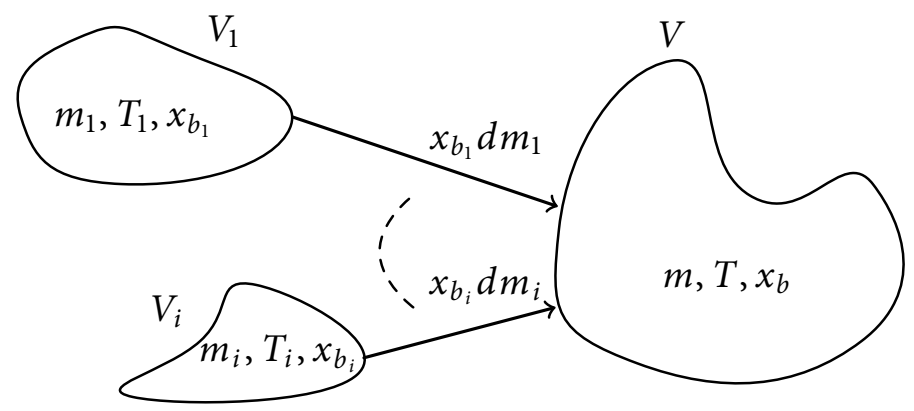

Figure 4.6: Conservation of mass and energy.

masses of respective species, i.e., equal to the right hand side of (4.54). The differential $d\left(m x_{b}\right)$ can also be written:

$$
d\left(m x_{b}\right)=x_{b} \sum_{i} d m_{i}+m d x_{b}
$$

where the chain rule is applied, and the summation of all incoming masses $d m=\sum_{i} d m_{i}$ is introduced. Combining (4.54) and (4.55), the mass concentration differential vector $d x_{b}$ for a number of species in a perfectly mixed container is:

$$
d x_{b}=\sum_{i} \frac{\hat{x}_{b_{i}}-x_{b}}{m} d m_{i}
$$

where $m$ is the total mass in the control volume. Since the gas composition in the control volume is not affected by the outflowing masses, $\hat{x}_{b_{i}}$ is used instead of $x_{b_{i}}$. The concentration vector $\hat{x}_{b_{i}}$ can be expressed:

$$
\hat{x}_{b_{i}}= \begin{cases}x_{b_{i}} & \text { When } i \text { is into the mixer }\left(d m_{i}>0\right) \\ x_{b} & \text { When } i \text { is out from the mixer }\left(d m_{i} \leq 0\right)\end{cases}
$$

where $x_{b_{i}}$ is the mass fraction of the incoming fluid $i$, and $x_{b}$ is the mass fraction in the control volume. The differential (4.56) expressed in air/fuel ratio $\lambda$ can be combined with the vector (4.35) to get:

$$
d x_{b}=\sum_{i} \frac{x_{b}\left(\hat{\lambda}_{i}\right)-x_{b}(\lambda)}{m} d m_{i}
$$

where

$$
\hat{\lambda}_{i}= \begin{cases}\lambda_{i} & \text { When } i \text { is into the mixer }\left(d m_{i}>0\right) \\ \lambda & \text { When } i \text { is out from the mixer }\left(d m_{i} \leq 0\right)\end{cases}
$$

according to the flow directions. 


\subsubsection{Lambda Concentration Differential $d \lambda$}

The differential of the mass fraction vector can be written:

$$
d x_{b}=\frac{d x_{b}}{d \lambda} d \lambda
$$

and the derivative of (4.35) with respect to lambda is:

$$
\frac{d x_{b}}{d \lambda}=\frac{a-b(A / F)_{s}}{\left((A / F)_{s} \lambda+1\right)^{2}}
$$

where $a=(A / F)_{s} x_{a}$ and $b=S x_{f}$ are introduced for easier notation. The differential of the mass fraction, which was derived in (4.58), is combined with (4.35), (4.60), and (4.61) to get:

$$
\frac{1}{m} \sum_{i}\left[\frac{a \hat{\lambda}_{i}+b}{(A / F)_{s} \hat{\lambda}_{i}+1}-\frac{a \lambda+b}{(A / F)_{s} \lambda+1}\right] d m_{i}=\frac{a-b(A / F)_{s}}{\left((A / F)_{s} \lambda+1\right)^{2}} d \lambda
$$

which can be solved according to $d \lambda$ :

$$
d \lambda=\frac{1}{m} \sum_{i}\left[\frac{(A / F)_{s} \lambda+1}{(A / F)_{s} \hat{\lambda}_{i}+1}\left(\hat{\lambda}_{i}-\lambda\right)\right] d m_{i}
$$

where

$$
\hat{\lambda}_{i}= \begin{cases}\lambda_{i} & \text { When } i \text { is into the mixer }\left(d m_{i}>0\right) \\ \lambda & \text { When } i \text { is out from the mixer }\left(d m_{i} \leq 0\right)\end{cases}
$$

as presented earlier. In this case, the mass fraction differential which is a vector can be replaced with the lambda differential which is a scalar. It can also be noted that the stoichiometric matrix $S$, the mass concentration vector of air $x_{a}$ and fuel $x_{f}$ do not appear in (4.63). The differential depends only on the stoichiometric air/fuel ratio $(A / F)_{s}$.

\subsubsection{Partial Derivatives of Gas Property Functions}

In the state equations, which will be presented in (4.67), the partial derivatives with respect to lambda for the thermodynamic quantities $u$ and $R$ appear. These partial derivatives can be expressed using the derivative of the mass concentration vector (4.61) which can be rewritten:

$$
\frac{d x_{b}}{d \lambda}=\frac{(A / F)_{s}}{(A / F)_{s} \lambda+1}\left(x_{a}-x_{b}\right)
$$

using (4.35). The partial derivatives of internal energy $u$, and specific gas constant $R$ of the frozen mixture are written:

$$
\begin{aligned}
& \frac{\partial u}{\partial \lambda}=\sum_{i} u_{i} \frac{d x_{b, i}}{d \lambda}=\frac{(A / F)_{s}}{(A / F)_{s} \lambda+1}\left(u_{a}\left(T_{a}\right)-u(T, \lambda)\right) \\
& \frac{\partial R}{\partial \lambda}=\sum_{i} R_{i} \frac{d x_{b, i}}{d \lambda}=\frac{(A / F)_{s}}{(A / F)_{s} \lambda+1}\left(R_{a}-R(\lambda)\right)
\end{aligned}
$$


where $u_{a}$ is the internal energy, $T_{a}$ is the temperature, and $R_{a}$ is the gas constant of the ambient air. When the mass fraction of the ambient air changes, these constants need to be updated.

\subsubsection{State Equations}

The differentials of the ideal law (4.51), the energy conservation equation (4.50), and the change in air/fuel ratio can be summarized in the following state equation differentials:

$$
\begin{aligned}
V d p-m T \frac{\partial R}{\partial \lambda} d \lambda-m R d T & =R T d m \\
m\left(c_{v} d T+\frac{\partial u}{\partial \lambda} d \lambda\right) & =d E-u d m \\
d \lambda & =\frac{1}{m} d \Lambda
\end{aligned}
$$

where

$$
\begin{aligned}
d m & =\sum_{i} d m_{i} \\
d E & =\sum_{i} h_{i} d m_{i} \\
d \Lambda & =\sum_{i}\left[\frac{(A / F)_{s} \lambda+1}{(A / F)_{s} \hat{\lambda}_{i}+1}\left(\hat{\lambda}_{i}-\lambda\right)\right] d m_{i}
\end{aligned}
$$

are introduced. Differential $d m$ is the total change of mass in the volume, $d E$ is the energy contribution of the incoming fluids, and $d \Lambda$ is the air/fuel ratio contribution. The air/fuel ratio, as previously defined, is:

$$
\hat{\lambda}_{i}= \begin{cases}\lambda_{i} & \text { When } i \text { is into the mixer }\left(d m_{i}>0\right) \\ \lambda & \text { When } i \text { is out from the } \operatorname{mixer}\left(d m_{i} \leq 0\right)\end{cases}
$$

according to the flow directions. In (4.50), $d Q$ and $d \tilde{W}$ are assumed to be zero, i.e., a perfectly insulated container with no applied external work. State variables for the control volume are chosen as pressure $p$, temperature $T$ and air/fuel ratio $\lambda$. In Figure 4.7 the exchange of mass, energy, and lambda according to (4.68) are showed.

\subsubsection{Variation in Ambient Absolute Humidity}

To handle variation in absolute humidity, the mass fraction vector of ambient air $x_{a}$ in (4.35) and (4.65) needs to be updated. The absolute humidity is calculated using the moist air model presented in Buck (1981). The humidity affects the stoichiometric air/fuel ratio $(A / F)_{s}$, the internal energy of air $u_{a}\left(T_{a}\right)$, and the gas constant of air $R_{a}$ which appear in (4.35), (4.66), (4.68), and (5.26). Since these quantities are defined as constants, the gas properties are updated instantaneous in all thermodynamic components. This results in transients when the absolute humidity of the ambient air changes. For the gas 


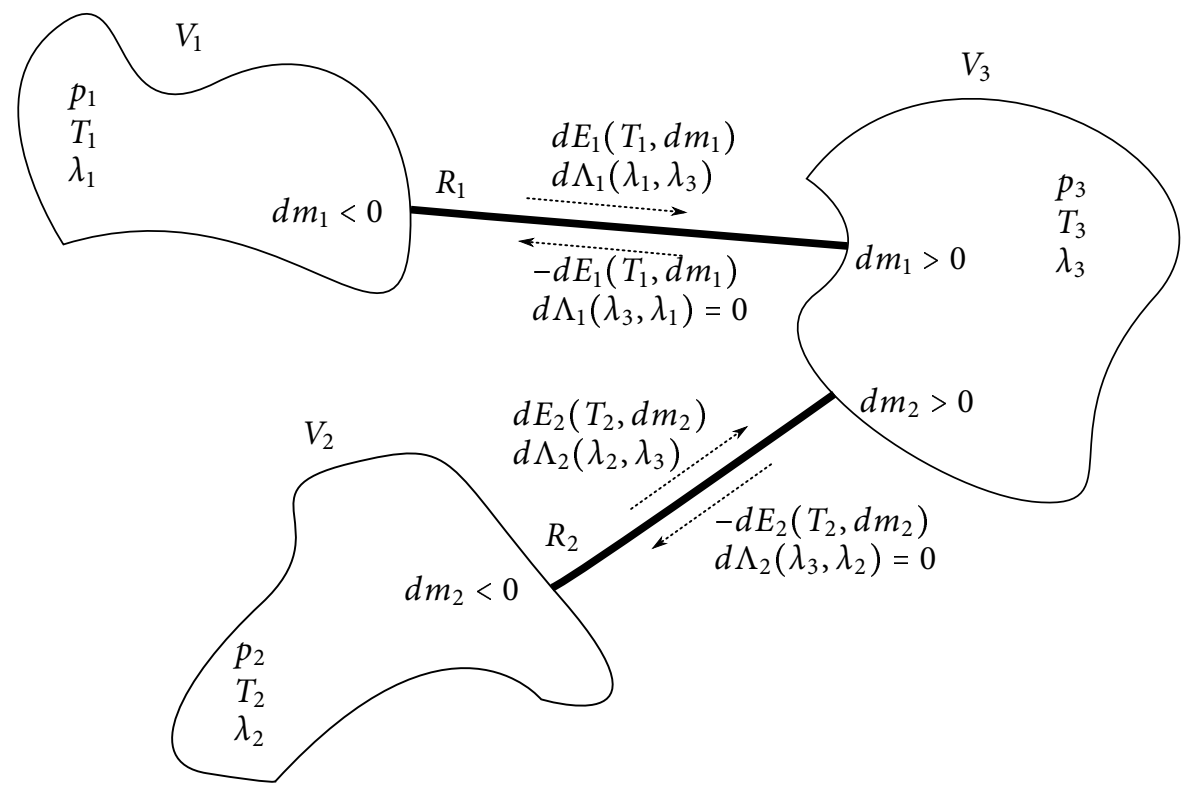

Figure 4.7: In the figure, three control volumes, together with two flow restrictions are presented. The mass flows through the restrictions have the directions from volumes $V_{1}$, and $V_{2}$ to volume $V_{3}$. The flow variables through the restrictions are the mass flow, the enthalpy flow, and the lambda flow according to (4.68). 
turbine application, this phenomenon is negligible because of the high throughput speed compared to the changes in the ambient conditions. The reason is that the ratio between the control volumes and the mass flow is small, i.e., the gas exchange is fast in the control volumes.

\subsection{Conclusion}

In the chapter, fundamental thermodynamic concepts are presented that can be used to describe a gas medium used in a combustion process. In the present study, these concepts are implemented in the gas turbine package - GTLib used to model an industrial gas turbine, which will be presented further in Chapter 5 . The central part of the chapter is the development of a control volume model, where an exhaust gas is described using the three state variables: (i) pressure $p$, (ii) temperature $T$, (iii) and air/fuel ratio $\lambda$. The ambient air, which is used in the combustion, can handle different amounts of humidity through an adjustment of the mass fraction of water. The framework handles hydrocarbon fuels with a specified number of individual substances. To model atmospheric air in a control volume, the $\lambda$ variable needs to be specified large, i.e., preferably infinitely large, but a large number is sufficient in practice. When the air/fuel ratio $\lambda$ is used as a state variable instead of the mass fraction of substances the total number of state variables is decreased.

The advantage with using the presented thermodynamic concept is the ability to integrate the combustion process into the model. During the combustion, the mass concentrations of species in the incoming air are changed, since species of carbon dioxide and water are produced under the consumption of oxygen. The number of these constructed species depends on the hydrocarbon fuel where the reaction formulas are represented by the stoichiometric matrix. The mass fraction of species in the exhaust gas is specified with the state variable air/fuel ratio $\lambda$. For this procedure, the assumptions are: (i) the combustion is lean (i.e., $\lambda>1$ ), (ii) the exhaust gas is frozen in composition (i.e., no dissociation effects between the species occur), and (iii) no pure substances can be introduced throughout the gas path. The first assumption is not a problem in a gas turbine application, since an excess of oxygen is available. The second assumption can be a problem if the flame temperature is too high. It is shown that for temperatures above $1500 \mathrm{~K}$, the thermodynamic properties of the fluid start to change. For a temperature of $2000 \mathrm{~K}$, the error in heat capacity $c_{p}$ is about $8 \%$ against a calculation where dissociation effects are considered. For the temperature of $1500 \mathrm{~K}$, the error is lower than $2 \%$. The third assumption states that no pure substances can be introduced into the exhaust gas since this will destroy the air/fuel ratio. This means that, e.g., water steam cannot be injected into the gas media throughout the gas path. 



\section{Chapter 5}

\section{GTLib - Thermodynamic Gas Turbine Modeling Package}

The objective of the chapter is to introduce the thermodynamic gas turbine modeling package GTLib, which is used to construct a physical based gas turbine model. The gas turbine model constructed in GTLib can be used for: (i) performance analysis, (ii) control issues, and (iii) as a base for further investigations when an FDI-system is constructed. When a model based diagnosis approach is used for the FDI-system design, it is crucial to have diagnosis statements based on good models. Roughly speaking, diagnosis tests based on good models give better diagnosis performance, i.e., increase the fault detectability and reduce the false alarm probabilities than when models with lower accuracy are used. Therefore, a good idea is to have diagnosis tests in the FDI-system based on the knowledge from the model used for performance analysis.

An important part of GTLib is the gas model which is used in the components along the engine's gas path to calculate the thermodynamic properties of the fluid. Using GTLib, the two main benefits are achieved: (i) an overall reduction in the gas turbine model equations compared to the reference gas turbine model, and (ii) the ability to achieve a systematic procedure to automatically construct test quantities when designing the FDI-system.

\section{Outline of the Chapter}

In Section 5.1, an overview of a typical appearance of the performance characteristics of a gas turbine is presented. The performance characteristics for the compressor and the turbine utilize the concept of corrected parameters which will be explained in the section. In Section 5.2, a small example that emphasize the difference in results between the gas description in GTLib and SITLib (developed by SIT) is shown. In the control volume example, a transient in the ambient air composition of substances is evaluated for the two cases. In Section 5.3, the implementation of GTLib at a high level is presented. Here, the gas model and its implemented thermodynamic functions together with the 
other gas turbine components are shown. In Section 5.4, an overview of the designed gas turbine model is presented.

\subsection{Gas Turbine Performance Characteristics}

A simple approach to describe the performance of a gas turbine component, e.g., the compressor, is to assume that the isentropic efficiency $\eta_{i s}$ is constant. In, e.g., Hadik (1990), the isentropic efficiency is considered to be $\eta_{i s}=0.87$. A more sophisticated method to describe the performance of gas turbine components is to use look-up tables of corrected parameters. The advantage with using corrected parameters in the look-up tables is the ability to describe the performance in other operating conditions than at the measured reference conditions. The corrected parameters are collected in nondimensional groups which have a background in dimensional analysis (Buckingham, 1914). A relation between the non-dimensional groups is presented in, e.g., Dixon and Hall (2010); Saravanamuttoo et al. (2001); Heywood (1988); Volponi (1999), and is reviewed here:

$$
[\Pi, \underbrace{\eta_{i s}}_{\eta_{\text {is }}^{*}}]=f(\underbrace{\frac{m_{\text {flow }} \sqrt{T_{01} R \gamma}}{D^{2} p_{01}}}_{m_{\text {flow }}^{*}}, \underbrace{\frac{n D}{\sqrt{T_{01} R \gamma}}}_{n^{*}})
$$

where $n$ is the shaft speed, $D$ is the impeller diameter, $\Pi$ is the pressure ratio, $m_{\text {flow }}$ is the mass flow of air, $R$ is the specific gas constant, $\gamma$ is the heat capacity ratio, $T$ is the temperature, $p$ is the pressure, and $\eta_{i s}$ is the isentropic efficiency. The subscript in, e.g., $T_{01}$ denotes stagnation temperature at the inlet of the component and the subscript will be removed in the following section to get simpler notation. The function arguments are corrected parameters and will be denoted with $(*)$ in the sequel. For a specific gas turbine, the impeller diameter is fixed so $D$ in (5.1) can be neglected. Normalized quantities of the corrected parameters can be constructed by multiplication of a non-dimensional constant to get:

$$
\begin{aligned}
m_{\text {flow }, \text { norm }}^{*} & =100 \frac{m_{\text {flow }}}{m_{\text {flow, ref }}} \frac{p_{\text {ref }}}{p} \sqrt{\frac{T}{T_{\text {ref }}} \frac{R}{R_{\text {ref }}} \frac{\gamma}{\gamma_{\text {ref }}}} \\
n_{\text {norm }}^{*} & =\frac{n}{n_{\text {ref }}} \sqrt{\frac{T_{r e f}}{T} \frac{R_{r e f}}{R} \frac{\gamma_{\text {ref }}}{\gamma}} \\
\eta_{\text {is,norm }}^{*} & =\frac{\eta_{\text {is }}}{\eta_{\text {is,ref }}} \\
C_{T, \text { norm }}^{*} & =\frac{C_{T}}{C_{T, \text { ref }}} \sqrt{\frac{R}{R_{\text {ref }} \frac{\gamma}{\gamma_{\text {ref }}}}}
\end{aligned}
$$

where ( $r e f)$ denotes the value at the reference state. In the last expression, the flow capacity notation is introduced where $C_{T}=\frac{m_{\text {flow }} \sqrt{T}}{p}$. The reference parameters must be given together with the look-up tables which are described herein. 


\subsubsection{Compressor Map}

The performance of the compressor is described by look-up tables of corrected and normalized parameters. The variables given by the map are the normalized mass flow of air, and the isentropic efficiency according to:

$$
\begin{aligned}
m_{\text {flow,norm }}^{*} & =f_{1, \eta}\left(\Pi, n_{\text {norm }}^{*}, \alpha\right) \\
\eta_{\text {is,norm }}^{*} & =f_{1, \Gamma}\left(\Pi, m_{\text {flow }, \text { norm }}^{*}, \alpha\right)
\end{aligned}
$$

where the function in (5.1) is extended with the angle $\alpha$ of the inlet guide vanes (IGVs).

The IGVs are used to change the inlet angle of the mass flow to obtain as high efficiency as possible regardless of the load. Since the machine is 2-shafted, the IGVs depend directly on the shaft speed of the gas generator and are therefore built into the look-up tables. Thus, they can be removed explicitly from (5.3). With IGVs, the compressor can also cover a wider operating range since the surge line of the compressor is moved. In Figure 5.1, the isentropic efficiency and the normalized speed lines are plotted versus the pressure ratio and the corrected normalized mass flow. In the figure, also the normalized speed lines are plotted versus the normalized mass flow.

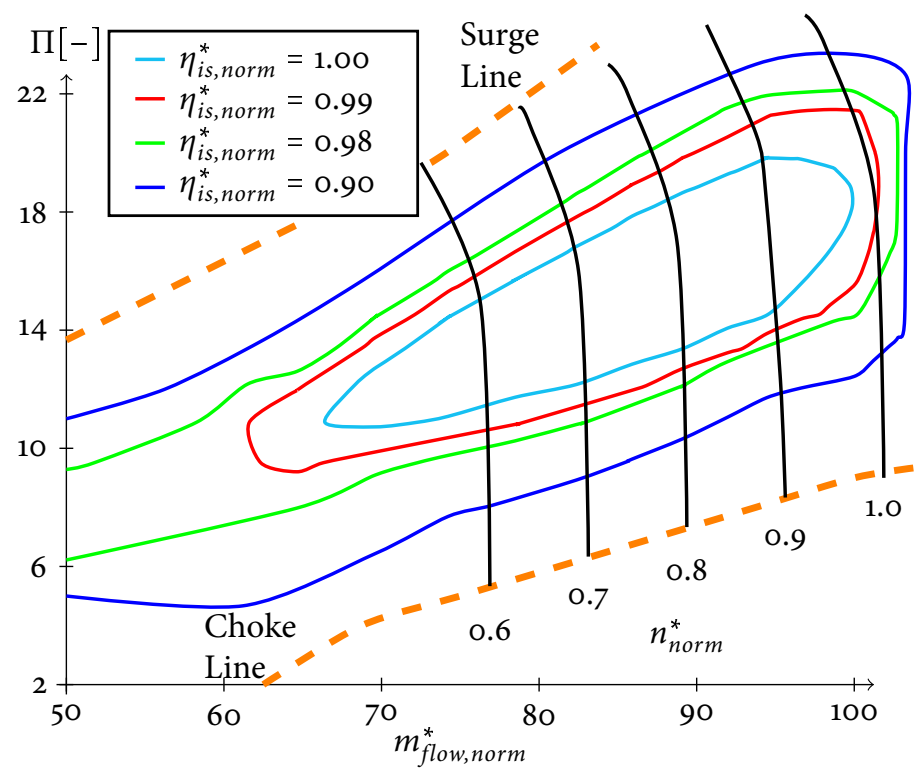

Figure 5.1: In the figure, a typical appearance of the performance characteristics of a compressor is shown. In the compressor map, the efficiency $\eta_{i s, n o r m}^{*}$ and the normalized mass flow $m_{\text {flow, norm }}^{*}$ are viewed for different pressure ratios $\Pi$ and normalized speeds $n_{\text {norm }}^{*}$. The surge line and the choke line are also shown in the figure. 


\subsubsection{Turbine Map}

The performance of the turbine is described in the same way as the compressor, i.e., using of normalized variables and look-up tables. Here, the calculated variables are the turbine flow capacity $C_{T}$ and the isentropic efficiency $\eta_{i s}$ according to:

$$
\begin{aligned}
C_{T, \text { norm }}^{*} & =f_{i, \eta}\left(\Pi, n_{\text {norm }}^{*} \frac{n_{\text {ref }}}{\sqrt{T_{\text {ref }}}}\right) \\
\eta_{\text {is,norm }}^{*} & =f_{i, \Gamma}\left(\Pi, n_{\text {norm }}^{*} \frac{n_{\text {ref }}}{\sqrt{T_{\text {ref }}}}\right)
\end{aligned}
$$

where $T_{r e f}$ and $n_{\text {ref }}$ from (5.2b) are neglected. Subscript $i=2,3$ represents the compressorturbine and the power-turbine respectively. In Figure 5.2, an example of a turbine map is viewed. In the figure, typical appearances of corrected and normalized isentropic efficiency, and turbine flow capacity are plotted versus the pressure ratio.

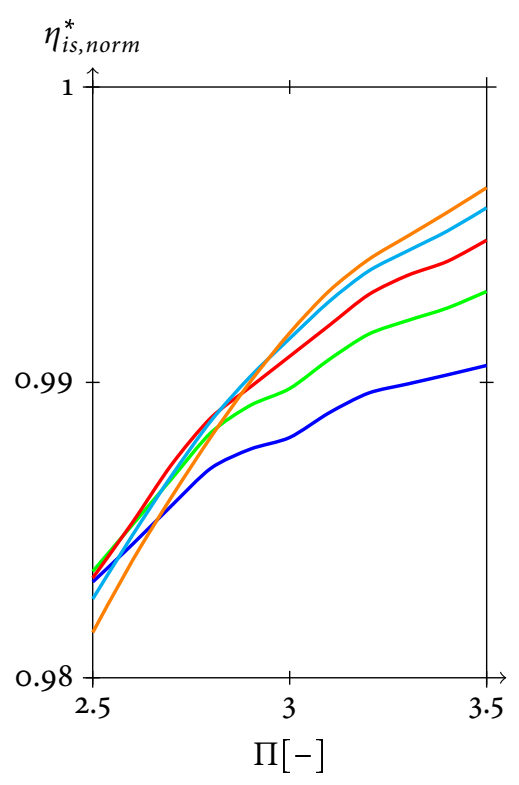

(a) Turbine efficiency.

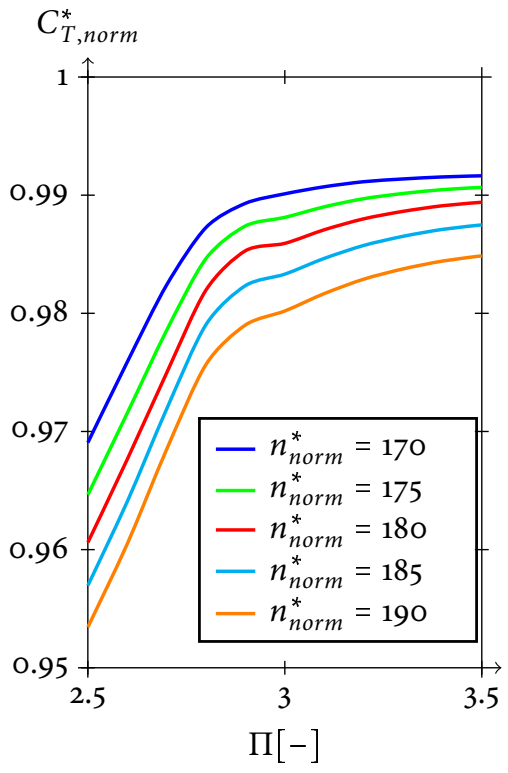

(b) Turbine flow capacity.

Figure 5.2: In the figure, a typical appearance of the performance characteristics of a turbine is shown. In the turbine maps, the efficiency $\eta_{i s, n o r m}^{*}$ and the normalized flow capacity $C_{T, n o r m}^{*}$ are calculated for different pressure ratios $\Pi$ and normalized speeds $n_{\text {norm }}^{*}$. 


\subsection{Variation in Ambient Absolute Humidity}

To achieve high model accuracy, one factor that can affect the performance estimations is the humidity in the ambient air. This phenomenon is often neglected since it is assumed that the change in absolute humidity does not vary significantly in an industrial gas turbine application. In Mathioudakis and Tsalavoutas (2002), an investigation is performed where it is shown how humidity influences the performance analysis for an industrial gas turbine. In the case study, it is argued that a large change in absolute humidity affect the performance analysis negative which can result in a case when a not correct diagnosis statement is taken. When experimental data is considered, a large deviation (on daily basis which shows a variation of about 2 per cent points) is present for the flow capacity of the compressor. When the compensation for the absolute humidity is considered in the evaluation, the undesirable daily variations in the flow capacity deviation are significantly reduced.

When the absolute humidity in the ambient air changes, also the constants which depend on the air, e.g., (i) the mass fraction vector of air $x_{a}$ (ii) the air/fuel ratio $(A / F)_{s}$ and (iii) and thermodynamic properties such as $u_{a}$ and $R_{a}$ need to be updated. These constants affect, e.g., the mass fraction of exhaust gas in (4.35), and partial derivatives in (4.66). Thus, when a compensation of the absolute humidity is desired the thermodynamic constants must be updated to maintain the correct exhaust gas mixture given by the air/fuel state variable.

For models constructed in GTLib, all the thermodynamic constants are updated immediately in all gas turbine components. This results in an error during transients in the ambient air composition which Example 5.1 should symbolize. For the gas turbine application, this phenomenon is negligible because of the high throughput speed compared to the changes in the ambient conditions. The ratio between the control volumes and the mass flow is small, i.e., the gas exchange is fast in the control volumes.

\section{Example 5.1}

Before the gas turbine performance model is presented, a good idea is to introduce a small simulation example that shows the main difference in gas properties between a thermodynamic system which uses the GTLib package and a system which uses SITLib package (Modelica Media). In Figure 5.3, such system is constructed (for the two cases) which consists of: (i) a source $S_{1}$, (ii) a sink $S_{2}$, (iii) a control volume $V_{10}$, and (iv) two pressure losses $d p_{1}$ and $d p_{2}$. The same type of models is used in the two simulation cases, and a step is injected in the ambient conditions such as temperature $T$ and relative humidity $\varphi$. In the example, it is interesting to compare how the gas properties in the volume $V_{10}$ are affected when the temperature and relative humidity have changed. To show this phenomenon, it is assumed that the incoming volume mass is much less then the available control volume mass in $V_{10}$, i.e., it takes long time to change all the mass in the control volume. In Figure 5.4, the temperature $T$, the pressure $p$, the specific gas constant $R$, the specific enthalpy $h$, the density $d$, and the relative humidity $\varphi$ of the control volume $V_{10}$ are viewed for the two simulation cases.

In the figure, it can be seen that during transients in ambient conditions, gas proper- 


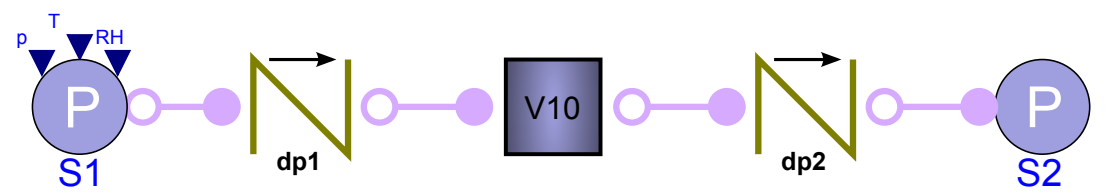

Figure 5.3: In the figure, a thermodynamic system with a control volume $V_{10}$, a gas source $S_{1}$, a gas sink $S_{2}$, and two pressure losses $d p_{1}, d p_{2}$ is presented. The input signals to the system are the pressure $p$, the temperature $T$, and the relative humidity $\varphi$ of the ambient air. It is assumed that the pressure in the gas source is higher than in the gas sink which gives a mass flow direction to the right in the figure.

ties such as specific gas constant, specific enthalpy, and density change instantaneously in GTLib, except for the temperatures in subfigure 5.4(a). This is because $(A / F)_{s}$ and $x_{a}$ in (4.35) are changed directly according to the ambient conditions, so they are updated simultaneously in all components in the model. When the transients have declined, the gas properties converge to the same values for the two simulation cases.

In the example, the incoming mass is much less than the mass in the control volume which can be seen as an extreme case. For the real gas turbine application, the throughput speed for the gas turbine is high so the error due to transients in ambient conditions is not a problem. The ratio between the control volumes and the mass flow is small.

\subsection{GTLib Components}

The developed gas turbine package GTLib can be used when a gas turbine model is constructed. The advantage with GTLib is the ability to build up a model which can be used for performance analysis and as a base when diagnosis test quantities used in an FDI-system are constructed. An important part of GTLib is the gas model (Section 5.3.3) where the thermodynamic relationships presented in Chapter 4 are implemented. These relationships are then introduced in the control volume model presented in Section 5.3.4. In the following subsections, the components of: the compressor, the turbine, and the combustor are presented.

\subsubsection{Global Environment Model}

To simulate the gas turbine model, a global environment component (GEC) needs to be defined. In the GEC, the mass fractions of the ambient air $x_{a}$, the hydrocarbon fuel $x_{f}$, and the stoichiometric matrix (4.33) are specified. When these parameters are specified, the stoichiometric air/fuel ratio $(A / F)_{s}$ can be calculated according to (4.36). All thermodynamic gas turbine components use the constants defined in the GEC.

The gas model in its original design can only handle fuel and air gases with a fix concentration of substances. To imitate more realistic environment conditions, a moist 


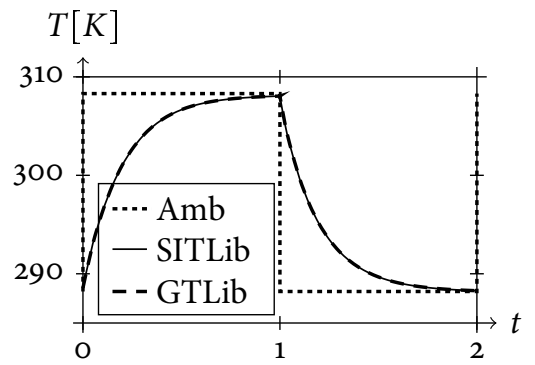

(a) Temperature

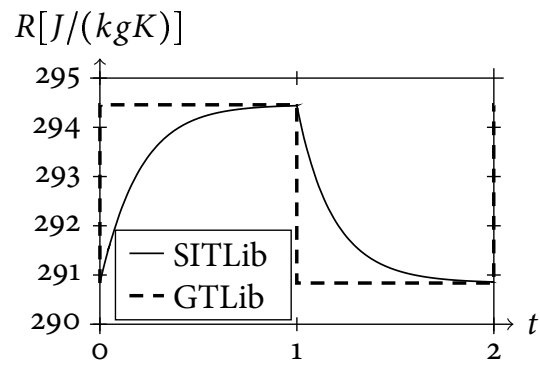

(c) Gas constant

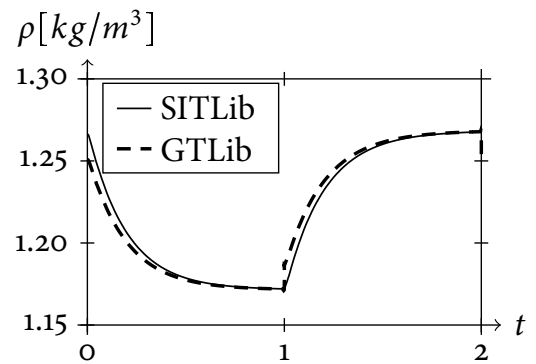

(e) Density

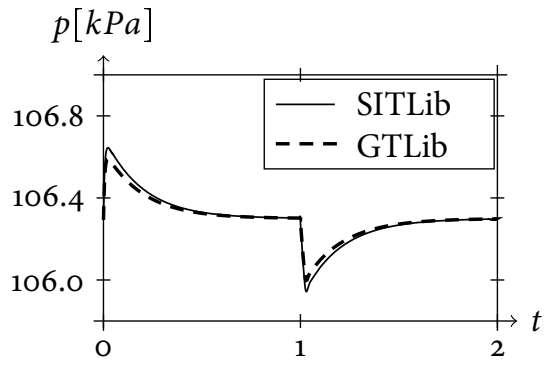

(b) Pressure

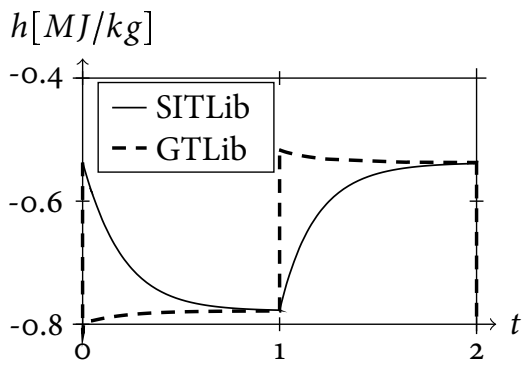

(d) Enthalpy

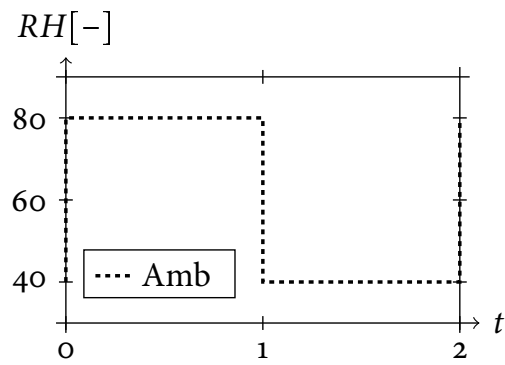

(f) Relative humidity

Figure 5.4: In Figure (a), a step in temperature $T$ and relative humidity $R H$ of the ambient air is introduced at time 0,1 , and 2. The experiment is performed for the two test cases where GTLib (dashed line) and SITLib (solid line) are used in the model presented in Figure 5.3. In the figures, the thermodynamic properties of the gas in the control volume $V_{10}$ are shown. The gas properties in all figures mismatch during the transient but the effect on the temperature is very small. The mismatch during a transient depends on an instantaneous change in the gas properties in GTLib, e.g., the gas constant $R$ goes from $\approx 294$ to 291 immediately. In the real gas turbine application, this phenomenon is not a problem since the mass flow is large compared to the size of the control volumes. 
air model (Buck, 1981) is introduced when the mass fraction vector $x_{a}$ of the ambient air is calculated. When the ambient air vector is updated, all constants where the ambient air properties are used need also to be updated, e.g., in (4.35), (4.66), and (5.33). With this procedure, the gas properties are updated immediately in all gas turbine components. This results in an error during transients in the ambient air composition which Example 5.1 symbolizes. For the gas turbine application, this phenomenon is negligible because of the high throughput speed compared to the changes in the ambient conditions. The ratio between the control volumes and the mass flow is small, i.e., the gas exchange is fast in the control volumes.

\section{Moist Air Model}

To describe the amount of water steam in the ambient air, the moist air model presented in Buck (1981) is adopted here. The amount of water is then used to describe the whole $x_{a}$ vector and implemented in the function humidAirCalc $\left(p_{0}, T_{0}, R H\right)$ given the input variables: pressure $p_{0}$, temperature $T_{0}$, and relative humidity $R H$ of the ambient air.

In the ambient gas model, the saturation pressure of water is described by Buck (1981). This expression is used in the meteorological context and has high accuracy in the region of -80 to $50^{\circ} \mathrm{C}$. The saturation pressure of water is described by:

$$
p_{\left(\mathrm{H}_{2} \mathrm{O}\right)_{s}}=6.1121 \cdot\left(1.0007+3.46 \cdot 10^{-3} p\right) \exp \left(\frac{17.502 T_{0}}{240.97+T_{0}}\right)
$$

where the ambient temperature $T_{0}$ is expressed in Celsius and the absolute pressure $p$ is expressed in bar. The saturation pressure of water $p_{\left(\mathrm{H}_{2} \mathrm{O}\right)_{s}}$ is expressed in hectopascal. The relative humidity $\varphi$ (which is known) is defined:

$$
\varphi=100 \frac{p_{\mathrm{H}_{2} \mathrm{O}}}{p\left(\mathrm{H}_{2} \mathrm{O}\right)_{s}}
$$

Thus, it is possible to calculate the partial pressure $p_{\mathrm{H}_{2} \mathrm{O}}$ of water vapor using (5.5). Here, it is assumed that the moist air consists of dry air and water steam, so the partial pressure of dry air $p_{a i r}$ is simply equal to the difference in atmospheric pressure $p$ and the partial pressure of water vapor $p_{\mathrm{H}_{2} \mathrm{O}}$, i.e., $p=p_{\mathrm{H}_{2} \mathrm{O}}+p_{\text {air }}$. This, together with ideal gas law (4.42a) gives an expression for the mass fraction of water according to:

$$
x_{\mathrm{H}_{2} \mathrm{O}}=\frac{p_{\mathrm{H}_{2} \mathrm{O}} R_{\text {air }}}{p_{\mathrm{H}_{2} \mathrm{O}} R_{\text {air }}+p_{\text {air }} R_{\mathrm{H}_{2} \mathrm{O}}}
$$

Since the mass fraction of substances in the dry air is known, the mass fraction of the moist air is also determined. When the moist air medium is known, it is possible to calculate thermal properties such as enthalpy and heat capacities as a function of temperature and air/fuel ratio throughout the gas path. It can be noted that a change in the absolute humidity in the ambient air affects the stoichiometric air/fuel ratio for a given hydrocarbon fuel. How the amount of water depends on the ambient condition such as relative humidity and temperature can be seen in Table 5.1.

It can be seen in the table that the amount of water in the ambient air increases drastically with temperature and humidity. In the medium model, the ambient conditions 
Table 5.1: Mass of water vapour, in gram, for $1 \mathrm{~kg}$ moist air at datum pressure, at temperature $T$, and relative humidity $\varphi$.

\begin{tabular}{lccc}
\hline $\mathbf{T}\left(C^{o}\right)$ & $\varphi=40 \%$ & $\varphi=60 \%$ & $\varphi=80 \%$ \\
\hline 15 & 4.21 & 6.33 & 8.45 \\
20 & 5.78 & 8.69 & 11.61 \\
25 & 7.85 & 11.80 & 15.78 \\
30 & 10.53 & 15.85 & 21.20 \\
35 & 13.99 & 21.08 & 28.22 \\
\hline
\end{tabular}

affect the gas properties immediately in every gas turbine component. This depends on the fact that the ambient variables are declared as global and all medium models use these global variables when the mass fraction of species in the ambient air is calculated. The ambient air concentration is then used when the actual gas properties are calculated.

\subsubsection{Connectors}

In Modelica, information between components is shared through connection points that are called connectors. In a connector, there are basically two types of variables which are defined either as a flow or a non-flow variable. In a connection point, flow variables are summed to zero, and non-flow variables are set equal.

With this approach, the flow variables are identified from (4.68) and Figure 4.7 to be: $d m, d E$, and $d \Lambda$. The summation in (4.68) is performed for the number of flows in each connector which is the same as the number of connected components. In general, each non-flow variable is specified in the control volume and each flow variable is calculated in the restrictions between the control volumes. The considered variables, which are used in the connectors are summarized in Table 5.2.

Table 5.2: In the table, the connector variables used in the GTLib package are presented. These variables are either defined as a flow or a non-flow variable. The flow variables are summed to zero, and the non-flow variables are set equal in the connector points.

\begin{tabular}{lll}
\hline Type & Variable & Description \\
\hline non-flow & $p$ & pressure \\
flow & $d m$ & mass flow \\
non-flow & $h$ & enthalpy \\
flow & $d E$ & enthalpy flow \\
non-flow & $\lambda$ & air/fuel ratio \\
flow & $d \Lambda$ & air/fuel ratio flow \\
\hline
\end{tabular}




\subsubsection{Gas Model}

The gas model (shown in Figure 4.5) is accessible in each component in the GTLib package where the state of the fluid is calculated. Given the state variables: pressure $p$, temperature $T$, and air/fuel ratio $\lambda$ all the other thermodynamic properties can be calculated, e.g., the enthalpy $h$ and the heat capacity $c_{p}$. The gas model consists of two parts: (i) an equation based part, and (ii) a function based part. The equation based part consists of the fundamental equations for an ideal gas and the function based part is used for calculation of thermodynamic properties, typically described by look-up tables which are provided by the Modelica standard library. The tabulated data is the well known NASA polynomial coefficients summarized in McBride et al. (2002).

In Modelica, flexible models can be defined due to the object oriented nature of the language. Therefore, the gas model is divided into: (i) a mandatory part, and (ii) an optional part. In the mandatory part, only the most important thermodynamic quantities are calculated given the state variables. The mandatory part consists of the thermodynamic relations:

$$
\begin{aligned}
& p=\rho R T \\
& u=h-R T \\
& h=h(T, \lambda) \\
& R=R(\lambda)
\end{aligned}
$$

where the mandatory quantities: enthalpy $h$, gas constant $R$, internal energy $u$, and density $\rho$ are calculated using the equations in (5.8). The mandatory part can be extended with a number of predefined functions which take the gas state variables as input arguments. The optional functions which can be utilized in the gas model are described in the remaining part of the present section.

\section{Mass Fraction Calculation in Exhaust Gas}

The function lambda2mass $(\lambda)$ makes a conversion between the air/fuel ratio $\lambda$ and the mass fraction vector $x_{b}$ of the exhaust gas. This function is central in the gas model since it is called everywhere when the gas properties are calculated. The function evaluate (4.35) and is reviewed here:

$$
x_{b}(\lambda)=\frac{(A / F)_{s} \lambda x_{a}+S x_{f}}{(A / F)_{s} \lambda+1}
$$

where $(A / F)_{s}$ is the stoichiometric air/fuel ratio, $S$ is the stoichiometric matrix (e.g., (4.33)), $x_{a}$ is the mass concentration of the ambient air, and $x_{f}$ is the mass concentration of the fuel. These parameters are either defined as constants or calculated in the global environment component (Section 5.3.1). When the global environment component is utilized it is possible to update the thermodynamic properties of the ambient air during the simulation. 


\section{Enthalpy}

The enthalpy function $\mathrm{h}(T, \lambda)$ makes the calculation according to $(4.43 \mathrm{a})$ and is reviewed here:

$$
h(T, \lambda)=\sum_{i} h_{i}(T) x_{b, i}(\lambda)
$$

where $h_{i}$ represents the standardized enthalpy (calculated according to the NASA polynomials and given in (4.19)) for substance $i$ for a given temperature. The input variables to the function are the gas temperature $T$, and the air/fuel ratio $\lambda$ of the gas. The output of the function is the enthalpy of the gas mixture.

\section{Entropy}

The entropy function $s(p, T, \lambda)$ is calculated based on the standardized entropy $s_{i}^{o}(4.21)$ of an ideal gas for each substance $i$. The entropy $s_{i}$ of an ideal gas is then calculated according to (4.23) and is reviewed here:

$$
s_{i}(T, p)=s_{i}^{o}(T)-R_{i} \ln \left(\frac{p}{p^{o}}\right)
$$

where the standardized entropy $s_{i}^{o}(T)$ and pressure $p^{o}$ is given for the datum state. The entropy for the mixture is then calculated using a summation of the substances weighted with the mass fraction of each substance:

$$
s(T, p, \lambda)=\sum_{i} s_{i}\left(T, p_{i}\right) x_{b, i}(\lambda)
$$

which can be simplified according to the derivation in (4.44). The data that is included in the absolute entropy vector is calculated according to the NASA polynomials. The input variables to the function are the pressure $p$, the gas temperature $T$, and the air/fuel ratio $\lambda$ of the exhaust gas. The output of the function is the entropy of the gas mixture.

\section{Isentropic Temperature}

For an ideal compression or expansion thermodynamic process the entropy in (5.11) is constant (see Figure 2.1). To calculate, e.g., the isentropic temperature $T_{2 s}$, the entropy function should be inverted. The function $T_{-} s(p, s, \lambda)$ calculates the temperature $T$ of an isentropic compression (or expansion process) for the input variables pressure $p$, entropy $s$, and air/fuel ratio $\lambda$. The function invert the entropy expression in (5.11) numerically using a number of iterations until:

$$
s_{1}-s_{2}\left(p_{2}, T_{2 s}, \lambda_{2}\right)<\varepsilon
$$

is small enough for the iteration temperature parameter $T_{2 s}$ when the isentropic process goes from state 1 to $2 s$. 


\section{Specific Gas Constant}

The function $\mathrm{R}(\lambda)$ calculates the specific gas constant for the exhaust gas. For each substance $i$ of the gas, the specific gas constant $R_{i}$ (in mass basis) is tabulated in the standard Modelica package. The gas constant is simply calculated according to $R_{i}=\tilde{R} / M_{i}$, where $M_{i}$ is the mole mass of the substance $i$ and $\tilde{R}$ is the universal gas constant. The specific gas constant of the gas mixture is calculated according to $(4.43 \mathrm{~b})$ and is reviewed here:

$$
R(\lambda)=\sum_{i} R_{i} x_{b, i}(\lambda)
$$

where $x_{b, i}$ is the mass fraction of substance $i$.

\section{Heat Capacity with Constant Pressure}

The functions c_p $(T, \lambda)$ calculate the heat capacity (for a constant pressure process) of the exhaust gas. The input variables to the function are the gas temperature $T$ and the air/fuel ratio $\lambda$. The heat capacity is calculated according to $(4.43 \mathrm{C})$ and reviewed here:

$$
c_{p}(T, \lambda)=\sum_{i} c_{p, i}(T) x_{b, i}(\lambda)
$$

where $x_{b, i}$ is the mass fraction of substance $i$.

\section{Heat Capacity with Constant Volume}

The functions $c_{-} \mathrm{v}(T, \lambda)$ calculate the heat capacity (for constant volume process) of the exhaust gas. The input variables to the function are the gas temperature $T$ and the air/fuel ratio $\lambda$. Since the ideal gas assumption is made, the heat capacity is calculated according to:

$$
c_{v}(T, \lambda)=c_{p}(T, \lambda)-R(\lambda)
$$

where $R$ is the specific gas constant.

\section{Isentropic Exponent}

The function gamma $(T, \lambda)$ calculates the isentropic exponent $\gamma$. The input variables to the function are the gas temperature $T$, and the air/fuel ratio $\lambda$. The isentropic exponent is the ratio between $c_{p}$ and $c_{v}$ which is calculated according to:

$$
\gamma=\frac{c_{p}(T, \lambda)}{c_{v}(T, \lambda)}
$$

where heat capacities functions $c_{-} \mathrm{p}(T, \lambda)$ and $c_{-} \mathrm{v}(T, \lambda)$ are called. 


\subsubsection{Control Volume}

The governing state equations of the control volume component are:

$$
\begin{aligned}
V \frac{d p}{d t} & =d m R T+m\left(R \frac{d T}{d t}+T \frac{\partial R}{\partial \lambda} \frac{d \lambda}{d t}\right) \\
m c_{v} \frac{d T}{d t} & =d E-u d m-m \frac{\partial u}{\partial \lambda} \frac{d \lambda}{d t} \\
m \frac{d \lambda}{d t} & =d \Lambda
\end{aligned}
$$

where the flow quantities are defined as:

$$
\begin{aligned}
& d m=\sum_{i} d m_{i} \\
& d E=\sum_{i} d E_{i}=\sum_{i} h_{i} d m_{i} \\
& d \Lambda=\sum_{i} d \Lambda_{i}=\sum_{i}\left[\frac{(A / F)_{s} \lambda+1}{(A / F)_{s} \hat{\lambda}_{i}+1}\left(\hat{\lambda}_{i}-\lambda\right)\right] d m_{i}
\end{aligned}
$$

and the calculations of $d E_{i}$ and $d \Lambda_{i}$ are performed by the pressure loss component that connects the control volumes according to Figure 4.7. The summations in (5.18) are performed in the control volume. The air/fuel ratio $\hat{\lambda}_{i}$ is:

$$
\hat{\lambda}_{i}= \begin{cases}\lambda_{i} & \text { When } i \text { is into the control volume }\left(d m_{i}>0\right) \\ \lambda & \text { When } i \text { is out from the control volume }\left(d m_{i} \leq 0\right)\end{cases}
$$

The partial derivatives (4.66), according to the air/fuel ratio are calculated as:

$$
\begin{aligned}
& \frac{\partial u}{\partial \lambda}=\frac{(A / F)_{s}}{(A / F)_{s} \lambda+1}\left(u_{a}-u\right) \\
& \frac{\partial R}{\partial \lambda}=\frac{(A / F)_{s}}{(A / F)_{s} \lambda+1}\left(R_{a}-R\right)
\end{aligned}
$$

where the derivation of the partial derivative of the mass fraction function (4.65) is used together with (4.35). The subscript $a$ represents the property of the ambient air. The total mass $m$ in the control volume (with volume $V$ ) is described by:

$$
m=V \rho
$$

where $\rho$ is the density which is defined in the gas model in Section 5.3.3.

\subsubsection{Compressor}

The compressor component consists of: (i) a mechanical, and (ii) a thermodynamic part since the energy is transformed from mechanical to thermodynamic energy through a compression. The compressor has ports where cooling air is tapped, used to decrease 
thermal stresses (caused by heat) in the first blade of the turbine. The ports used for cooling air are placed at enthalpy ratio $r_{h_{i}} \in[0,1]$, where $r_{h_{i}}=0$ represents the low pressure side and $r_{h_{i}}=1$ represents the high pressure side. During the compression phase, the temperature $T$ and pressure $p$ of the gas increase. For an isentropic compression the entropy is constant (shown in, e.g., Figure 2.1). Thus, when the compression is ideal it is possible to calculate the discharge temperature (or enthalpy) at the output of the compressor. This is done according to the previous defined function T_s and can be written:

$$
T_{2 s}=T_{s}\left(p_{2}, s_{2}, \lambda_{1}\right)
$$

When the isentropic temperature $T_{2 s}$ increase, also the enthalpy increase. Thus the change in enthalpy $\Delta h$ can be written:

$$
\Delta h=\frac{h\left(T_{2 s}, \lambda_{1}\right)-h\left(T_{1}, \lambda_{1}\right)}{\eta_{i s}}
$$

where $\eta_{i s}$ is the isentropic efficiency defined by a look-up table (see, e.g., Figure 5.1). The air/fuel ratio is not changed during the compression, thus is $\lambda_{1}=\lambda_{2}$. The thermodynamic power $P_{\text {thermo }}$ can be written:

$$
P_{\text {thermo }}=\left[\sum_{i}\left(1-r_{h_{i}}\right) m_{\text {flow }, r_{h_{i}}}\right] \Delta h
$$

where $r_{h_{i}} \in[0,1]$ is the enthalpy ratio, index $r_{h_{i}}$ denotes the port position, and the summation is over all ports. A mass flow $m_{\text {flow, } r_{h_{i}}}$ which leaves the compressor has a negative sign. The mechanical power $P_{\text {mech }}$ is:

$$
\begin{aligned}
& P_{\text {mech }}=\frac{P_{\text {thermo }}}{\eta_{m}} \\
& P_{\text {mech }}=\frac{d \varphi}{d t} \sum_{i} \tau_{i}
\end{aligned}
$$

where $\eta_{m}$ is the mechanical friction constant, $\varphi$ is the shaft angle, and $\tau_{i}$ is the applied torque. The mass and the energy balances are written:

$$
\begin{aligned}
& 0=\sum_{i} m_{\text {flow }, r_{h_{i}}} \\
& 0=\sum_{i} H_{\text {flow }, r_{h_{i}}}-\left[\sum_{i} r_{h_{i}} m_{\text {flow }, r_{h_{i}}}\right] \Delta h
\end{aligned}
$$

The performance parameters such as isentropic efficiency $\eta_{\text {is }}$ and incoming mass flow $m_{\text {flow }}$ are calculated according to the look-up tables defined in (5.3), together with the normalized corrected parameters in (5.2). The normalized corrected parameters are all calculated at the low pressure side of the compressor.

\subsubsection{Turbine}

The turbine component is analogue with the compressor component, except that no cooling air is extracted from the gas expansion. Instead, compressed air from the compressor 
is injected in the first turbine blades to protect the material from high temperature stresses. Since the look-up tables are valid for the hot gas (before cooling air is injected) it is necessary to mix the hot and cool gases after the characteristic calculations are made.

Since exhaust gases and cooling air have different air/fuel ratio the mixed gas has the air/fuel ratio $\lambda_{\text {mix }}$ according to:

$$
\lambda_{\text {mix }}=\frac{\left(\lambda_{\text {inj }} m_{\text {flow }, \text { inj }}+\lambda_{e} m_{\text {flow }, e}\right)+\lambda_{\text {inj }} \lambda_{e}(A / F)_{s}\left(m_{\text {flow }, i n j}+m_{\text {flow }, e}\right)}{\left(\lambda_{e} m_{\text {flow }, \text { inj }}+\lambda_{\text {inj }} m_{\text {flow }, e}\right)(A / F)_{s}+\left(m_{\text {flow }, i n j}+m_{\text {flow }, e}\right)}
$$

where subscript inj denotes the compressed air, and subscript $e$ denotes the hot exhaust gas. Equation (5.26) is calculated according to (4.41). When the air/fuel ratio of the mixture is determined the enthalpy change $\Delta h$ is:

$$
\Delta h=\left[h\left(T_{4 s}, \lambda_{\text {mix }}\right)-h_{\text {mix }}\right] \eta_{i s}
$$

where $h_{\text {mix }}$ is the enthalpy of the mixture, and $T_{4 s}$ is the isentropic temperature after the gas expansion. The mass flow of the exhaust gas $m_{f l o w, e}$ is calculated:

$$
C_{T}=m_{\text {flow }, e} \frac{\sqrt{T_{e}}}{p_{e}}
$$

where $C_{T}$ (and $\eta_{i s}$ ) are calculated according to the look-up tables defined in (5.4), together with the normalized corrected parameters in (5.2). The thermodynamic power $P_{\text {thermo }}$ is:

$$
P_{\text {thermo }}=-m_{\text {flow }, \text { mix }} \Delta h
$$

where $m_{\text {flow, mix }}=m_{\text {flow,inj }}+m_{\text {flow,e }}$. The mechanical power $P_{\text {mech }}$ is calculated according to (5.24). The mass and the energy balances are written:

$$
\begin{aligned}
& 0=m_{\text {flow,mix }}+m_{\text {flow,drain }} \\
& 0=H_{\text {flow,mix }}+H_{\text {flow,drain }}+m_{\text {flow,mix }} \Delta h
\end{aligned}
$$

where subscript drain denotes the low pressure side of the turbine.

\subsubsection{Combustor}

The combustion in a gas turbine is assumed to be a constant pressure process. For a constant pressure combustion process the sum of the reactant enthalpies (air and fuel) is equal to the sum of the product enthalpies (substances). Given the total enthalpy, the adiabatic flame temperature $T_{f}$ can be calculated according to (4.25). Since it is not necessary to determine the flame temperature $T_{f}$ explicitly, the enthalpy at the exhaust port of the combustor component is calculated according to:

$$
h_{e}=h_{a} \chi_{a}+h_{f}\left(1-\chi_{a}\right)
$$

where $h_{f}$ is enthalpy of fuel, $h_{a}$ is enthalpy of air, $h_{e}$ is enthalpy of the hot exhaust gas, and $\chi_{a}$ is the mass fraction of air. The mass fraction of air is:

$$
\chi_{a}=\frac{m_{\text {flow }, a}}{m_{\text {flow }, a}+m_{\text {flow }, f}}
$$


with the same subscripts as previous. The air/fuel ratio $\lambda$ of the exhaust gas is:

$$
\lambda=\frac{m_{\text {flow }, a} / m_{\text {flow }, f}}{(A / F)_{s}}
$$

directly according to the definition in (4.27). A pressure loss model through the combustor is defined according to Saravanamuttoo et al. (2001):

$$
P L F=\frac{2 A^{2} \rho_{1} \Delta p}{m_{\text {flow }, a}^{2}}
$$

where $P L F$ is the pressure loss factor which is constant, $\Delta p$ is the pressure drop, $A$ is the maximum cross-sectional area of the chamber, and $\rho_{1}$ is the density for the incoming air.

\subsubsection{Pressure Losses}

Two types of pressure loss components are implemented in GTLib. These pressure losses are: (i) a simple pressure loss component (5.35a), and (ii) a turbulent pressure loss component (5.35b) according to:

$$
\begin{aligned}
& m_{\text {flow }}=m_{\text {flow }, \text { ref }} \sqrt{\frac{\Delta p}{\Delta p_{\text {ref }}}} \\
& m_{\text {flow }}=A \sqrt{\frac{2 \rho_{1} \Delta p}{\xi}}
\end{aligned}
$$

here $\xi$ is a pressure loss factor, $\Delta p$ is the pressure loss, $p_{1}$ is the pressure of the upstream gas, $\rho_{1}$ is the density for the upstream gas, and subscript ref denotes reference values.

\subsection{Gas Turbine Model}

The main difference between the gas turbine model (described in the present chapter) and the reference gas turbine model (described in Section 2.3.1) is the description of the gas model. In the developed gas turbine model, the exhaust gas is specified through the air/fuel ratio $\lambda$ and in the reference gas turbine model the gas is specified through the mass fraction of species. A benefit with the model constructed in GTLib is the reduction of equations and state variables according to the air/fuel description. The reduction in model equations reduces the demanded simulation time by about 20 per cent. The model constructed in GTLib is not as general as the reference model constructed in SITLib since pure substances cannot be injected throughout the gas path, only the exhaust medium (with a air/fuel ratio $\lambda \in[1, \infty[$ ) can be injected.

The constructed gas turbine model consists of a number of standard components, e.g., control volumes, turbines, and valves which is shown in Figure 5.5. The gas turbine model and the reference model consist of the same type of components and have the same type connection to the environment. Thus, it is possible to simulate both models 
using the same simulation platform. The available instrumentation sensors measure: (i) pressures, (ii) temperatures, and (iii) shaft speeds throughout the gas path. Between the output of the compressor $\mathrm{Cl}$ and the output of the power turbine $\mathrm{T} 0$ there are no available measured gas path parameters. Since gas path parameters between these two points are important to supervise, it is necessary that the developed diagnosis tests can estimate those parameters.

When the gas turbine model is designed it can be used for performance analysis and as a base when diagnosis test quantities used in the FDI-system are constructed. Why the gas turbine model and not the reference model can be used for this purpose depends on the parsers which are developed. Since the Modelica Media package used in the reference model is very general, it can also be a challenge to develop parsers for this model. Thus, the developed parsers can only parse a subset of the Modelica language. The components in GTLib fulfill the specification of the parsers and can therefore be used to generate diagnosis test quantities. It will also be shown that the reference model has unobservable state variables which is not desirable when tests based on observers are constructed. The observability of the gas fraction in the reference model is also unclear. More about the test selection and construction procedure will be described in Chapter 6 .

\subsection{Conclusion}

In the chapter, a gas turbine modeling package - GTLib implemented in Modelica is presented. The GTLib package consists of two main parts: (i) a gas model, and (ii) a gas turbine component library. The gas model handles the calculation of thermodynamic properties of the fluid, and the gas turbine component library consists of the gas turbine components. Later on in the chapter, a gas turbine model is constructed from the components in the GTLib package. In the GTLib, the air/fuel ratio concept is introduced which reduces the number of equations and variables in the overall gas turbine model. The constructed model can be simulated together with the existing simulation platform. The gas turbine model can handle different changes in ambient conditions. These ambient conditions are the pressure, the temperature, and the relative humidity.

The benefit with using GTLib package is the reduction in model equations compared to the reference model constructed in SITLib. The accuracy of the two models is similar except for transients in absolute humidity of the ambient air. In GTLib, the air properties are updated simultaneously in all control volumes in the gas turbine model which gives the behavior. A disadvantage with GTLib is the loss in generality, here the only gases that are admitted to be used are the air and fuel. The consequence is that an injection of, e.g., pure oxygen somewhere in the gas path is not allowed. An advantage with GTLib is that the reduced number of equations gives a decreased simulation time when the simulation platform is simulated. The main propose with GTLib is that a diagnosis and supervision system can be constructed with the GTLib gas turbine model as a base. 


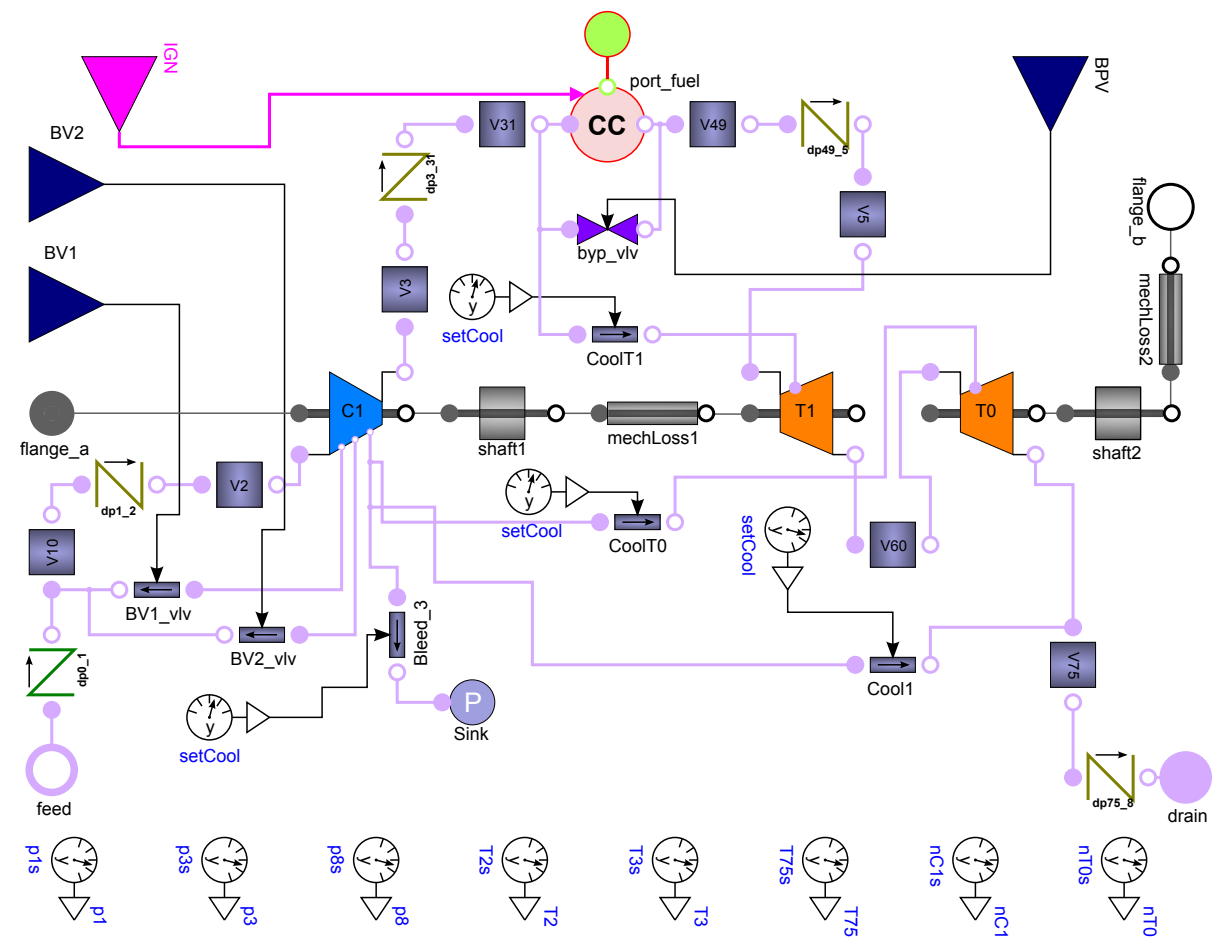

Figure 5.5: In the figure, a model of the 2-shafted gas turbine presented in Figure 2.4 is shown. The model consists of components from the GTLib package, e.g., control volumes, valves, and turbines. This model can be simulated together with the simulation platform viewed in Figure 2.5 since both gas turbine models have the same environment connections except for the gas sources. This model can be used for dynamic simulations exactly as for the reference model where the accuracy between the two models is high, but with a reduced simulation time. During start-up phases, the actuator signals BV1 and BV2 are used to control the bleed valves. The other actuator signals are IGN and BPV, where IGN control the ignition and BPV the bypass valve through the combustion chamber. The sensors measure pressure and temperature before and after the compressor $\mathrm{Cl}$, pressure and temperature after the power turbine $\mathrm{T} 0$, and the shaft speed of the gas generator and the power turbine. Between the output of the compressor and the power-turbine there are no measured gas path parameters. 


\section{Chapter 6}

\section{Modeling, Analysis, and Transformation of the Diagnosis Model}

The overall objective with the present work is to achieve a systematic method to construct an FDI-system from an available model used for performance analysis. The first step in the procedure is to design a diagnosis model where diagnosis concepts are introduced. To simplify the test quantity construction, the diagnosis model has only signal based connections to the environment (compared to the performance model which has physical based connections). In the diagnosis model design, the knowledge that is built in the performance model is transferred to the diagnosis model according to the object oriented nature of the Modelica language.

\section{Detection and Isolation of Faults}

Before the FDI-system is built, it is relevant to make some preliminary investigations how introduced faults, or component deteriorations, affect the fault detection and isolation performance. For the present gas turbine application, is it possible to detect and isolate: (i) faults in the input and output signals, and (ii) drifts in the performance parameters with the given set of measurement sensors? By definition, when a fault $f_{i}$ is detectable it is possible to distinguish between a non faulty system behavior and a faulty system behavior affected by the induced fault $f_{i}$. The detectability and isolability criteria depends on the particular sensor configuration where more sensors increase the diagnosis performance. The fault isolability requirements can be defined in a similar manner as for the fault detectability. Instead of distinguishing between a non faulty mode and a faulty mode, it should be possible to distinguish between two different faulty modes. For linear differential algebraic systems, the isolability is formally defined in Frisk et al. (2009) as a null-space calculation of the system matrix $H(p)$ in:

$$
H(p) x(t)+L(p) z(t)+F(p) f(t)=0
$$


where $x(t) \in \mathbb{R}^{n_{x}}$ represents the unknown variables, $z(t) \in \mathbb{R}^{n_{z}}$ represents the known signals, and $f(t) \in \mathbb{R}^{n_{f}}$ represents the unknown faults. The matrices: $H(p), L(p)$, and $F(p)$ are polynomial matrices in the differential operator $p=d / d t$, and they have an appropriate dimension. For an over-determined system, the number of rows in the matrices is larger than the dimension of $x$. In the linear case, the isolability properties can be determined through a null-space calculation of the system matrix $H$ together with the faulty mode $F_{j}$ which represents how fault $f_{j}$ affects the system. To check if $f_{i}$ is isolable from $f_{j}$, the null-space calculation should not be perpendicular to the faulty mode $F_{i}$ which is formally written:

$$
N_{H F_{j}}(p) F_{i}(p) \neq 0
$$

where $N_{H F_{j}}(p)$ is the null-space of $\left[H(p) F_{j}(p)\right]$. Thus, the fault $f_{i}$ is isolable from fault $f_{j}$ in (6.1) if, and only if (6.2) is satisfied. To check in practice if the system may be faulty, the linear filter:

$$
r(t)=\gamma N_{H}(p) L(p) z(t)
$$

is designed. The filter in (6.3) is called a consistency based residual generator where the design parameter $\gamma$ (weight factor) is a vector of suitable dimension. The residual generator should fulfil:

$$
\lim _{t \rightarrow \infty} r(t) \rightarrow 0
$$

when the system is in a non-faulty mode.

For nonlinear systems, it can be difficult to get an exact characterization of the model equation (i.e., based on consistency relations) which can be used when residual generators are constructed. In most cases when a residual generator is constructed, the system model together with the measurement equations are transformed into a number of smaller subsets where only the interesting faults are present see, e.g,. Blanke et al. (2003); Chen and Patton (1999); Nikoukhah (1994). The faults that are not present in the subset of equations are not detectable by the residual generator, i.e., theses faults are decoupled by the residual generator. A common method to construct residual generators is to design estimators based on state estimates, i.e., an observer design. In this class of residual generators, the state estimates of the observer is compared to the measurement signals to construct a residual generator. Residual generators based on state observers are presented in, e.g., Patton and Hou (1998); Martínez-Guerra et al. (2005); Svärd and Nyberg (2008).

To answer questions about detectability and isolability properties of a model in a general way, only the model structure can be considered (Blanke et al., 2003). For the gas turbine model considered herein, an analytic characterization of the model equations used for residual generators is not practical to find due to: (i) the size of the system, (ii) the nonlinear behavior of the model, and (iii) the look-up tables used for performance calculations. Thus, structural methods together with an observer design are considered when the test quantities (residual generators) used in the FDI-system are constructed.

\section{Outline of the Chapter}

The chapter starts with an historical overview of the gas turbine monitoring field where the estimation parameters (which are called health parameters) used in the Gas Path 
Analysis are presented. In Section 6.2, the diagnosis model is presented which introduces the faults and estimation parameters used for diagnosis. The following sections deal with the analysis of the diagnosis model. The investigations are: (i) the DAE-index analysis in Section 6.3, (ii) the structural analysis in Section 6.4, and (iii) the observability analysis in Section 6.5. In Section 6.7, a number of parsers used for an automatic extraction of the test equations are presented. These parsers are used to convert the Modelica models constructed in GTLib package into runnable Matlab code.

\subsection{Gas Turbine Monitoring}

In industrial gas turbines, deterioration in components throughout the gas path is commonly occurring and contributes to the overall performance degradation of the engine. Diagnosis and supervision of performance degradation in the application is a widely studied topic in the gas turbine monitoring research field, see, e.g., Aker and Saravanamuttoo (1989); Volponi et al. (2003); Doel (2003) where the performance parameters are estimated with different methods. When reliable performance estimations are available, it can be easier for the service engineers to efficiently plan service and maintenance of the gas turbine. In Aker and Saravanamuttoo (1989), it is investigated how compressor fouling affects the performance parameters using a linear fouling model. For a medium fouled compressor, the estimations appear to be reasonably accurate for the linear fouling model.

In papers Diakunchak (1992); Kurz and Brun (2001); Kurz et al. (2009), several mechanisms which cause degradation in gas turbines are presented. The major contribution of degradation mechanisms in an industrial gas turbine is fouling. The fouling is caused by small particles and contaminants in the air that are caught by the compressor. These particles increase the roughness of the rotor and stator surface. Another effect that results in performance degradation is the tip clearances which is a common diagnosis for aged gas turbines. Tip clearances denote an increasing gap between the rotating blades and the stationary casing. Fouling due to increased roughness can partially be restored by washing the compressor, while a component replacement is often needed for tip clearances. In the paper Brekke et al. (2009), compressor fouling in two different off-shore gas turbine applications is investigated. The fouling analysis showed that a considerable amount of contaminants appeared at the compressor inlet, at the inlet guide vanes, and at the first rotor stage of the compressor. In this case, the main contaminant in the samples was sodium-based salts which indicate that the gas turbine performance can be restored by a compressor wash.

In Diakunchak (1992), an analysis due to fouling is performed using experimental data from an industrial gas turbine site. In the paper, it is shown that the fouling of the compressor results in a $5 \%$ reduction in inlet mass flow and $1.8 \%$ reduction in compressor efficiency from the nominal values. This gives a reduction in the output power by about $7 \%$ and increases the heat rate of the fuel by about $2.5 \%$. 


\subsubsection{Gas Path Analysis}

One of the most famous and pioneering tool for gas turbine engine supervision and sensor diagnosis is the gas path analysis (GPA). The GPA algorithm can be summarized into the main steps: (i) measurement normalization, (ii) reference value generation, (iii) estimation of performance deviation, and (iv) diagnosis decision. This algorithm was introduced by Urban (1969) and an investigation of the method in a gas turbine engine application was presented in Urban (1972). The GPA method is based on thermodynamic relationships where one of the main objective is to estimate deterioration in gas path components from a number of measured sensor signals which are denoted as measurement deltas $(\Delta)$. In Urban (1969), a chart that contained the most commonly occurring gas turbine engine parameter interrelationships in a general matrix form was presented. These equations could be used to estimate steady state and transient variations in the performance parameters for an arbitrary gas turbine engine during most conceivable sets of input conditions. How the chart is calculated is re-printed in Giampaolo (2009) and the linear relationship between the measured signals and the engines health parameters is written (Volponi, 1994):

$$
\Delta Z=H \Delta h+e
$$

where $\Delta Z$ is the measured parameter deltas, $\Delta h$ is the deviation in performance, $H$ is the influence coefficient matrix presented in Urban (1969), and $e$ is the measurement noise. Elements in $\Delta h$ are typically: efficiencies $\Delta \eta$, and flow capacities $\Delta \Gamma$ of the gas path components such as compressors, turbines, and fans. Elements in $\Delta Z$ are typically: spool speeds $\Delta N$, temperatures $\Delta T$, and pressures $\Delta P$. The matrix $H$ can be divided into two parts: (i) an engine fault influence matrix $H_{e}$, and (ii) a sensor fault influence matrix $H_{s}$, where the previous defined matrix $H$ and health parameter $\Delta h$ in (6.5) are extended with the sensor fault dependencies.

The main objective with the gas path analysis framework is to estimate the performance deviation vector $\Delta h$. Depending on the size of the $H$ matrix, different approaches are relevant to consider. When engine's health is considered and not the sensor faults, the system (6.5) is often over-determined, i.e., the number of measurement deltas are larger than the number of performance parameter deltas. This results in an optimization problem where, e.g., least-square methods can be applied to solve the optimization problem. When also faults in sensors are considered in the equation system (6.5) the $\Delta h$ vector is extended with the faults and the matrix $H$ is extended with the influence of the faults. This results often in an under-determined system. For this case, the estimation procedure results in, e.g., minimization of some predefined cost function, or a maximization of the probability that gives the observed measurement. In Doel (1994) the commercial gas path analysis program TEMPER is presented. In TEMPER, it is assumed that the deviation in performance $\Delta h$ and the measurement noise $e$ are Gaussian distributed. To maximize this conditional probability distribution function is equivalent to minimize a quadratic form which has a weighted least-squares solution. 


\section{The Measurement Delta Vector}

The measurement delta vector $\Delta Z$ describes the deviation, from a nominal baseline in percent, for a number of known signals (or combination of known signals). These deviations (or deltas) are then collected in the $\Delta Z$-vector in (6.5). For example, the measurement delta $\Delta Z_{P}$ of the gas path parameter $\mathcal{P}$ is defined:

$$
\Delta Z_{\mathcal{P}}=100 \frac{\mathcal{P}^{*}-\mathcal{P}_{\text {nom }}^{*}}{\mathcal{P}_{\text {nom }}^{*}}
$$

where $\mathcal{P}^{*}$ is the corrected value according to the ambient standard conditions (e.g., the corrected speed $n^{*}=n /(\sqrt{T R \gamma})$ as described in $\left.(5.2 \mathrm{~b})\right)$, and $\mathcal{P}_{\text {nom }}^{*}$ is the nominal value under the chosen reference condition. The nominal value is typically calculated using a reference engine model together with an input signal $u$. The inputs can be, e.g., the mass flow of fuel, and/or the power generated by the application. A sketch of the calculation procedure is shown in Figure 6.1. The measurement deltas can directly be considered to detect abnormal changes in the supervised components according to deteriorations or sensor faults. The measurement deltas are utilized in, e.g., Ganguli and Dan (2004) where a recursive median filtering approach is used to recognize an abnormal change in the supervised components. When the measurement deltas are determined they can be used in (6.5) (for the linear case) to calculate the performance deviation $\Delta h$ vector using, e.g., some optimization method.

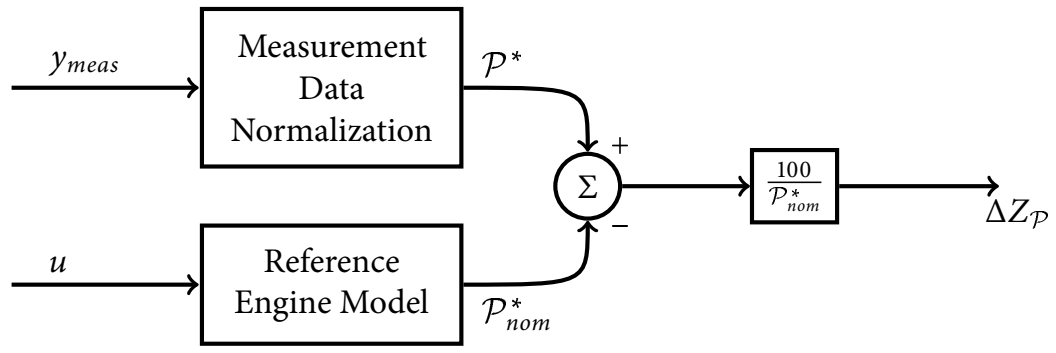

Figure 6.1: Measurement delta $\Delta Z_{P}$ calculation of the gas path parameter $P$, where $(*)$ denotes the corrected version of the parameter according to, e.g., (5.2) and Volponi (1999). The corrected nominal value $\mathcal{P}_{\text {nom }}^{*}$ is typically calculated using a reference engine model together with an input signal $u$.

\subsubsection{Engine Health Monitoring}

A common approach in the gas turbine diagnosis research field to capture performance degradation is to introduce a number of physical based quantities named health parameters. The motives to use those extra parameters are: (i) the health parameters are indicators for overhaul and maintenance, and (ii) the health parameters compensate for the deterioration in components which result in more reliable estimates of unmeasured signals used by, e.g., the controller. The health parameters were included in the performance deviation $\Delta h$ vector in the static equation system (6.5). A natural extension of the 
static model is to introduce the health parameters in a dynamic gas turbine model. As in the static case, these health parameters are typically corrections (or deviations from a nominal baseline) of efficiencies and flow capacities. The considered health deterioration appears in, e.g., the compressors, the turbines, the fans, and the nozzles. The introduced health parameters can be estimated with a number of techniques, see, e.g., Luppold et al. (1989); Kobayashi and Simon (2003); Borguet and Léonard (2008). In the first two papers, Kalman filters are utilized to estimate the health parameters directly. In the third paper, a nonlinear Kalman filter is used together with a quadratic optimization procedure. The optimization problem is studied to estimate the health parameter values which are then used in the Kalman filter to estimate the other state variables of the system.

Since performance degradation in the gas turbine is naturally occurring, it can be difficult to avoid false alarms when the diagnosis system does not compensate for the deterioration in components. This depends on the fact that the errors in the performance model get larger when the component deteriorations increase. This can result in estimated sensor values that differ too much from the measured sensor value which may trigger a false alarm (Kobayashi and Simon, 2003).

\subsection{Gas Turbine Diagnosis Model}

The objective with the diagnosis model is to specify the properties of the test quantities which are later on used when the FDI-system is designed. Using the diagnosis model, the ability to capture abnormal process behaviors using some extra estimation parameters is introduced. The considered abnormal process behaviors can be divided into the three general groups: (i) performance deterioration in components, (ii) component faults, and (iii) sensor and actuator faults. Component faults can be, e.g., a leakage in a valve or an increased friction in a bearing. In this chapter, the methodology to transform the diagnosis model into smaller subsets that are called test equations is introduced. Those test equations are then implemented and evaluated on experimental data in Chapter 7. A graphical representation of the diagnosis model, along with the input and output signals, is shown in Figure 6.2 and in Figure 6.3.

The gas turbine diagnosis model is a reduced and a simplified version of the reference gas turbine performance model which is developed by SIT and shown in Figure 2.5. The phrase reduced means that the number of equations and state variables in the model has decreased according to the utilization of the GTLib package. The phrase simplified means that some model simplifications have been done. In the diagnosis model, the following components are removed: (i) the bleed valves in the compressor, (ii) the bypass valve through the combustor, and (iii) the heat losses in components after the combustion (but heat losses in the combustor are still considered). The bleed valves are usually used during startup and shutdown phases (to avoid stall and surge in the compressor) and at full load operations these valves are fully closed. The bypass valve is usually used during partial base loads but does not affect the performance calculations significantly. Since it is assumed that the valves are closed it is possible to remove them from the model. Since the bleed valves are removed, the model is only valid during operational conditions, and are not valid during startup and shutdown operating modes (in Chapter 8 where startup 


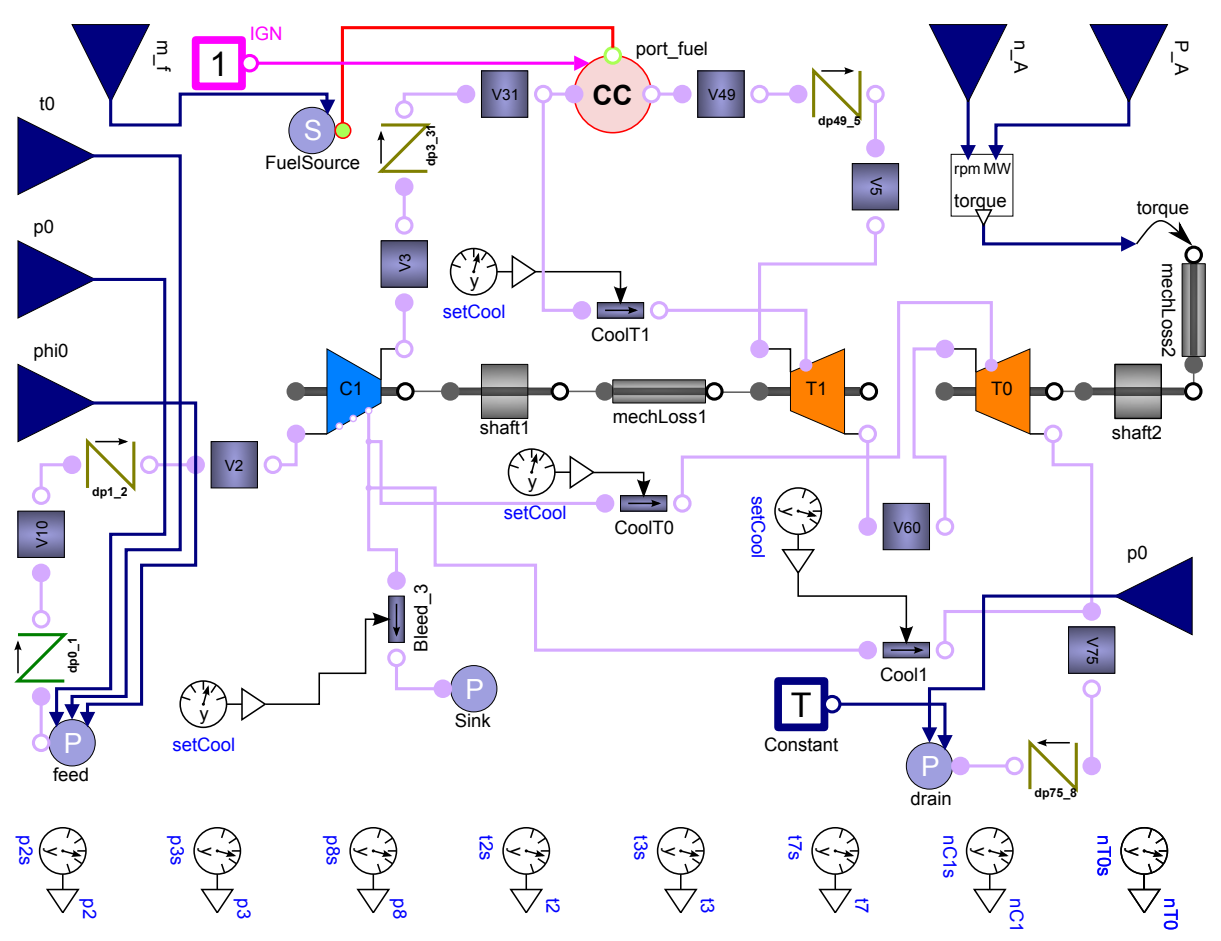

Figure 6.2: The figure gives an overview of the gas turbine diagnosis model. To get the diagnosis model, some of the components from the gas turbine model used for performance analysis are removed. These components are the bleed valves and the combustor bypass valve. Thus, the diagnosis model is valid only during operational conditions. The input signals to the diagnosis model are: (i) temperature $T_{0}$, pressure $p_{0}$, and relative humidity $\varphi_{0}$ of the ambient air, (ii) the mass flow of fuel $m_{f}$, and (iii) speed and power of the power-turbine T0. Since the model has no physical based connections to the environment, it is possible to simulate the diagnosis model with the given input signals. 
and shutdown operating modes are studied, these valves are introduced again).

\subsubsection{Input Signals}

All industrial gas turbines are equipped with a number of actuators and instrumentation sensors. The input signals are divided into two groups: (i) ambient air signals, and (ii) other signals. The first group of signals is: pressure $p_{0}$, temperature $T_{0}$, and relative humidity $\varphi_{0}$ of the ambient air. These signals are used to setup the surrounding ambient conditions, and used to calculate the concentration of substances in the ambient air using a moist air model, i.e., how much water steam that is mixed with dry air. The other group of signals is: mass flow of fuel $m_{f}$, power generated by the application $P_{A}$, and speed of the driven component $n_{A}$.

\subsubsection{Output Signals}

A number of measurement signals throughout the gas path is available for monitoring and control. The considered output signals are: (i) temperature $T_{2}$ and pressure $p_{2}$ before the compressor, (ii) temperature $T_{3}$ and pressure $p_{3}$ after the compressor, (iii) temperature $T_{7}$ and pressure $p_{8}$ after the power-turbine, and (iv) speed of gas generator $n_{C 1}$ and the power-turbine $n_{T 0}$.

\subsubsection{Health Parameters}

To capture degradation in efficiency and mass flow, health parameters in: (i) the compressor $\mathrm{Cl}$, and (ii) the compressor-turbine $\mathrm{Tl}$ are introduced in the diagnosis model. It is also possible to insert health parameters in the power-turbine T0, and in the inlet and outlet duct to detect abnormal pressure drops. Depending on the configuration, the considered health parameters are utilized in the performance equation:

$$
\begin{aligned}
& \eta_{i}=f_{i, \eta}(\ldots)+\Delta \eta_{i} \\
& \Gamma_{i}=f_{i, \Gamma}(\ldots)+\Delta \Gamma_{i}
\end{aligned}
$$

where $\eta_{i}$ is the efficiency, $\Gamma_{i}$ is the flow capacity, and $f_{i, j}$ represents the nominal characteristics. Subscript $i$ represents the components: C1, T1, or T0. In function $f_{i, j}$, the concept of corrected and normalized parameters are utilized according to (5.2)-(5.4). Since the component deterioration is assumed to be slow relative to the other gas turbine dynamics, constraints according to:

$$
\begin{aligned}
\Delta \dot{\eta}_{i} & =0 \\
\Delta \dot{\Gamma}_{i} & =0
\end{aligned}
$$

are added to the model for each health parameter. 


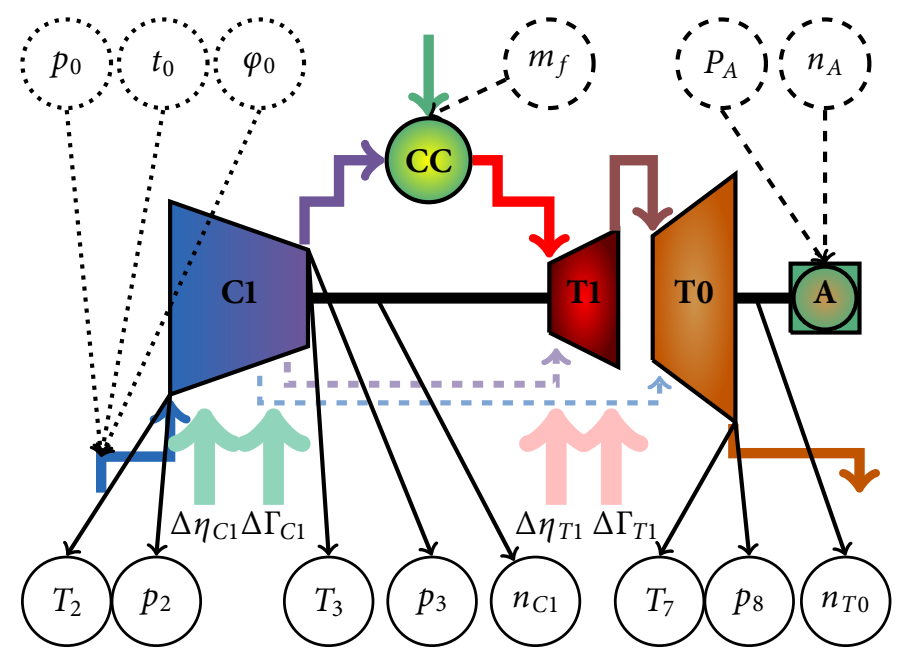

Figure 6.3: The gas turbine with the output signals (solid), the input signals (dashed), the input ambient signals (dotted), and health parameters (arrows) is shown in the figure. The secondary air flows, used to cool the first turbine blades, are shown with dashed arrows.

\subsubsection{Component Faults}

In the diagnosis model, faults in components can be introduced in the similar manner as for the health parameters. A component fault is, e.g., a leakage in a valve or an increased friction in a mechanical bearing of the shaft.

\subsubsection{Faults in Signals}

A faulty instrumentation sensor or actuator can be defined as a signal that shows an abnormal behavior. The abnormal behavior can be interpreted as, e.g., the sensor characteristic specified by the manufacture that is no longer maintained. The abnormal behavior can result in different kind of faulty behavior, e.g., these faulty behaviors can appear as:

- Abrupt changes - the faulty value changes behavior immediately.

- Incipient faults - the faulty value appears, and gradually increases in amplitude.

- Intermittent faults - the faulty value appears and disappears with a time interval.

- Bias faults - the faulty value is constant, i.e., the value has a constant offset.

In most cases, the faulty behavior of the sensor or actuator is unknown. In this case, the sensor signal can be modeled with an unknown variable $f_{i}$ added to the measured quantity. 


\section{Sensor Faults}

The sensor fault in the gas turbine model are introduced as:

$$
\begin{aligned}
y_{p_{1}} & =p_{1}+f_{p_{1}} \\
y_{p_{3}} & =p_{3}+f_{p_{3}} \\
\vdots & =\vdots \quad \vdots
\end{aligned}
$$

where $y_{i}$ is the known measurement signal, and $p_{i}$ is the measured quantity.

\section{Actuator Faults}

The faults in the input signals of the gas turbine model are introduced as:

$$
\begin{aligned}
& u_{m_{f}}=m_{f}+f_{m_{f}} \\
& u_{P_{A}}=P_{A}+f_{P_{A}} \\
& u_{n_{A}}=n_{A}+f_{n_{A}}
\end{aligned}
$$

where $u_{i}$ is the known input signal, and $p_{i}$ is the input quantity.

\subsubsection{Differential Algebraic Equation Form}

The diagnosis gas turbine model, with the added health parameters and sensor/actuator faults, has the general form:

$$
\begin{aligned}
F\left(\dot{z}, z, u+f_{u}\right) & =0 \\
y & =h(z)+f_{y} \\
z & =(x, \Delta)^{T}
\end{aligned}
$$

where $\Delta=\left(\Delta \eta_{C 1}, \Delta \Gamma_{C 1}, \Delta \eta_{T 1}, \Delta \Gamma_{T 1}\right)^{T}$ represents the unknown health parameters, $x$ consists of the unknown variables, $y$ consists of the known measurement signals, $u$ consists of the known input signals, $f_{s}$ consists of the unknown sensor faults, and $f_{a}$ consists of the unknown actuator faults. The functions $F$ and $h$ together with their arguments are vector valued functions with appropriate dimensions. Equation (6.11a) is a general mathematical description where the algebraic and the dynamic constraints are mixed, i.e., in a DAE-form. The expression in (6.11) is the starting point for the model analyses performed in the remaining part of the chapter.

\subsection{DAE-Index Analysis}

The objective with this section is to transform the system in (6.11) to a system in state space form:

$$
\begin{aligned}
\dot{z}_{1} & =f\left(z_{1}, u\right) \\
y & =h\left(z_{1}\right)
\end{aligned}
$$


where $z_{1}$ represents the state variables, $y$ represents the measurement signals, and $u$ represents the input signals. The functions $f$ and $h$ together with their arguments are vector valued functions with appropriate dimensions. A central concept in this section is the differential index (Hairer et al., 1991; Ascher and Petzold, 1998) of the system. The differential index of the system tells the minimum number of times the constraints must be differentiated to obtain a state space form.

\subsubsection{Differential Index Reduction}

The first step in the procedure is to check and reduce the differential index of the diagnosis model. After row and column permutations are performed, the DAE system in (6.11) can be written in the form:

$$
\begin{gathered}
{\left[\begin{array}{cc}
\bar{E} & 0 \\
0 & 0
\end{array}\right] \dot{\bar{z}}=\bar{f}(\bar{z}, u)} \\
y=h(\bar{z})
\end{gathered}
$$

where $u$ consists of the known actuator signals, $\bar{z}$ consists of the unknown variables, and $\bar{E}$ is a constant matrix which does not have full column rank (is singular). For this system, a state space form cannot be directly obtained before an index analysis is performed. In general, a model with a DAE index-1 (or 0) is easier to handle in practice than a system with a higher index (Ascher and Petzold, 1998). One way of handling this situation is to employ an index reduction technique and reduce the index of the system. A common method is to differentiate well chosen model equations a suitable number of times to obtain a low index DAE model. The constraints which are differentiated are removed from the model and treated separately. A disadvantage with the model reformulation is the numerical inabilities to satisfy the removed constraints which can appear. To handle the numerical problems techniques with, e.g., dummy derivatives (Mattsson and Söderlind, 1993) can be considered. To select which constraints that should be differentiated, Pantelides's algorithm (Pantelides, 1988) is considered. Using Pantelides's algorithm, also the differential index of the system can be determined. The input to the algorithm is the structural model (shown in Section 6.4) of the system. The index analysis shows that (6.13) is a DAE index-2 system, thus one derivation of selected algebraic constraints is necessary. When the equations, chosen by Pantelides's algorithm, are differentiated the diagnosis model (6.13) is written in the form:

$$
\begin{aligned}
{\left[\begin{array}{cc}
E_{1} & 0 \\
0 & 0
\end{array}\right]\left[\begin{array}{l}
\dot{z}_{1} \\
\dot{z}_{2}
\end{array}\right] } & =\left[\begin{array}{l}
f_{1}\left(z_{1}, z_{2}, u\right) \\
g_{1}\left(z_{1}, z_{2}, u\right)
\end{array}\right] \\
0 & =\left[\tilde{g}_{0}\left(z_{1}, z_{2}, u\right)\right]
\end{aligned}
$$

where $z_{1} \in \mathbb{R}^{n_{1}}, z_{2} \in \mathbb{R}^{m_{1}}, \operatorname{rank}\left(E_{1}\right)=n_{1}$, and $\operatorname{rank}\left(\frac{\partial g_{1}}{\partial z_{2}}\right)=m_{1}$ (at least locally). The algebraic constraints $\tilde{g}_{0}$, which are differentiated, are saved in $(6.14 \mathrm{~b})$.

\section{High Index Property of the Diagnosis Model}

The Mechanical Rotational library, which is a part of the Modelica standard package, share torque $\tau$ and angle $\varphi(5.24 \mathrm{~b})$ in connection points between rotational components. 
The index analysis shows that the high differential index property of the gas turbine model is a consequence of the angles dependencies in the connection points (angles are equal in a connection point). Pantelides's algorithm suggests to differentiate the equations in the form: $\varphi_{1}=\varphi_{2}, \varphi_{2}=\varphi_{3}, \ldots$ to obtain: $\dot{\varphi}_{1}=\dot{\varphi}_{2}, \dot{\varphi}_{2}=\dot{\varphi}_{3}, \ldots$. When the system is replaced with these new differentiated equations, the differential index of the system $(6.14 \mathrm{a})$ is 1 . The equations, before they are differentiated, are saved in the $\tilde{g}_{0}$ part. To get the quadratic $E_{1}$ matrix, e.g., QR-factorization or SVD-decomposition can be considered. Here, it is enough to replace the dynamic constraints: $\dot{\varphi}_{1}=\dot{\varphi}_{2}, \ldots, \dot{\varphi}_{n-1}=\dot{\varphi}_{n}$ with the algebraic constraints: $\omega_{1}=\omega_{2}, \ldots, \omega_{n-1}=\omega_{n}$ to get a quadratic $E_{1}$ matrix.

\section{Semi-Explicit Index-1 DAE Form}

The system in (6.14a) can be written in semi-explicit index-1 DAE form:

$$
\begin{aligned}
E_{1} \dot{z}_{1} & =f_{1}\left(z_{1}, z_{2}, u\right) \\
0 & =g_{1}\left(z_{1}, z_{2}, u\right) \\
y & =h_{1}\left(z_{1}, z_{2}\right)
\end{aligned}
$$

where the $E_{1}$-matrix is invertible, and the Jacobian $\partial g_{1} / \partial z_{2}$ is non-singular. This means that the algebraic constraint $g_{1}$ is invertible (at least locally), and can be solved for $z_{2}$ (Hairer et al., 1991; Ascher and Petzold, 1998). For the semi-explicit index-1 DAE system it is possible to distinguish between the differential variables $z_{1}$, and the algebraic variables $z_{2}$. This semi-explicit system can directly be transformed into a state space form through an inversion of the $E_{1}$ matrix and an elimination of the algebraic variable $z_{2}$ using the constraint $g_{1}$.

\section{State Space Form}

A state space form of the semi-explicit index-1 DAE system (6.15) is:

$$
\begin{aligned}
\dot{z}_{1} & =f_{2}\left(z_{1}, u\right) \\
y & =h_{2}\left(z_{1}, u\right)
\end{aligned}
$$

where the algebraic variable $z_{2}$ has been eliminated. This is possible since the Jacobian matrix $\partial g_{1} / \partial z_{2}$ of (6.15b) is, at least locally, non-singular. An observability analysis performed in Section 6.5 shows that the system (6.16) has unobservable modes. Thus, an observer cannot be constructed directly from the system (6.16) and further analysis of (6.15) is necessary to perform.

\subsection{Structural Analysis}

The objective with the structural analysis is to select relevant parts, and investigate fault detectability of the model. For nonlinear DAE systems, it can be difficult to get an exact characterization of the model equation which can be used when diagnosis tests are constructed. To have a structural analysis with higher accuracy, it is a good idea to specify as much detail as possible about the system. For the gas turbine engine, the 
gas flow has a given direction and the combustor is always turned on. This knowledge is utilized when the structural analysis is performed and gives a structural model that gets sparser. The structural model is a coarse model description where only the variable dependencies in each equation are considered. The analytic model is described by a matrix where each element has a true (1), or a false (0) value. How the variables affect the analytical equations are not considered in the analysis. Whether they affect the expression through, e.g., exponential, logarithmic, or using a look-up table, they still get the same variable dependencies in the structural model. When a specific variable is included in a specific equation, the matrix element belonging to the certain variable is (1). All these variable dependencies of the equations are collected in the structural model where the rows represent the equations and the columns represent the variables. When the model is in this form, the opportunity to develop fast algorithms for model analysis and especially for diagnosis purposes is available. The disadvantage is that only the best case solution is obtained.

\subsubsection{Dulmage-Mendelsohn Decomposition}

A method that can be useful when relevant subsets of model equations are chosen is the Dulmage-Mendelsohn (DM) decomposition, presented in Dulmage and Mendelsohn (1958). The DM decomposition is an equivalent description of a bi-partite graph, which specify the calculation chain of a system. The decomposition works on the structural model, and rearranges rows and columns to obtain the structure shown in Figure 6.4.

In the figure, the structural model is divided into the three separated parts: (i) an under-determined part $M^{-}$, (ii) an exactly-determined part $M^{0}$, and (iii) an overdetermined part $M^{+}$. These parts are described as:

(i) In the under-determined part, the number of equations in $M^{-}$is less than the number of variables in $X^{-}$. When the underlying analytical model can be simulated, this part never appears. The gas turbine diagnosis model used here does not involve this part and is therefore not considered in the analysis.

(ii) In the exactly-determined part, the number of equations in $M^{0}$ is equal to the number of variables in $X^{0}$. The exactly-determined part is divided into a number of components $a_{i}$. The components that consist of more than one equation are said to be strongly connected which means that the equations contain loops or cycles. For diagnosis purposes, the exactly-determined part of the model provides no extra information. Thus, this part of the model can be removed without loosing any redundancy.

(iii) In the over-determined part, the number of equations in $\mathrm{M}^{+}$is more than the number of variables in $X^{+}$. This indicates that redundancy is available and the degree of redundancy depends on the number of available measurement sensors. When diagnosis tests are constructed, different subsets of the $M^{+}$can be chosen. In Krysander et al. (2008), a class of these subsets is denoted Minimal structural over-determined sets (MSO sets). These subsets are easy to determine using the 


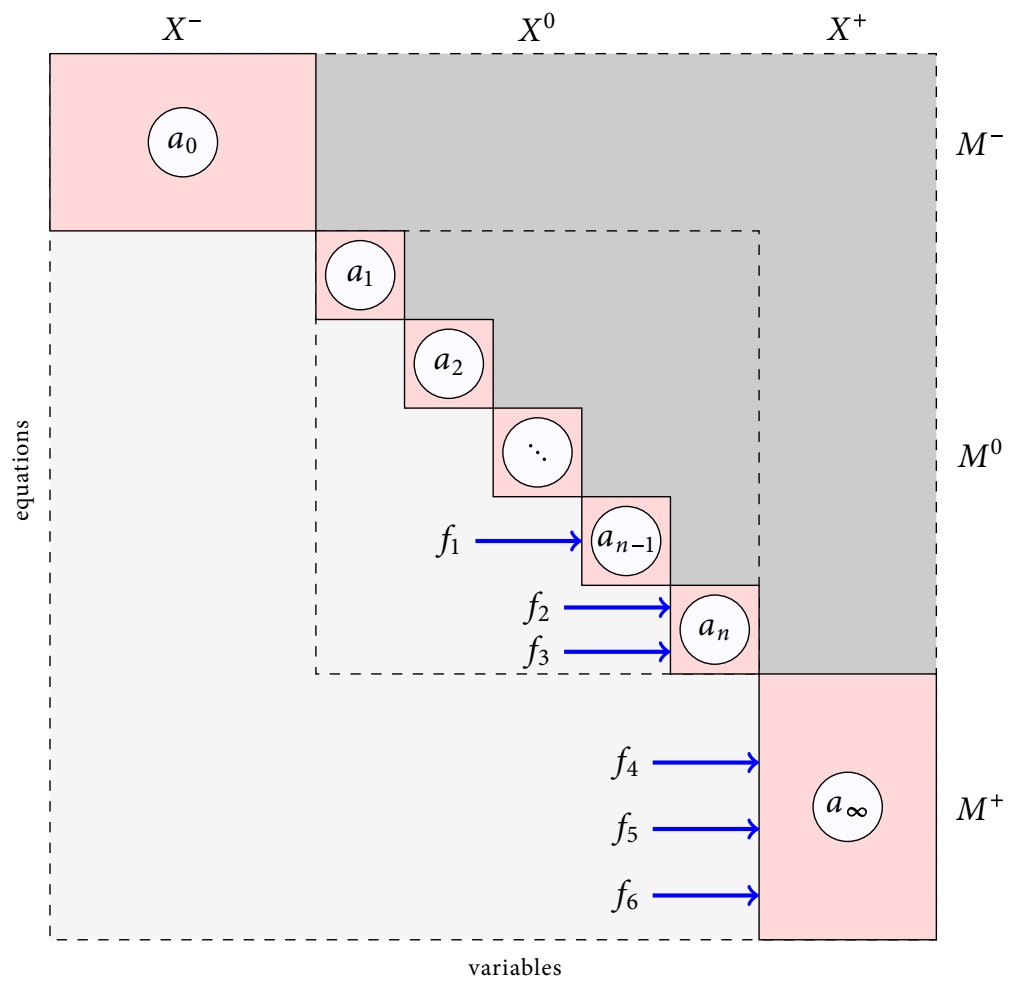

Figure 6.4: The figure shows a Dulmage-Mendelsohn decomposition of a general structural model where the rows represent the equations and the columns represent the variables. The light grey shadowed area consists of zero matrix elements. The matrix element in the darker grey area can either be zero or one. The elements that are left span the boxes $a_{0} \ldots a_{\infty}$. Injected faults $f_{1}, f_{2}, f_{3}$ are not detectable, but faults $f_{4}, f_{5}, f_{6}$ can possibly be detectable. 
proposed algorithm. The MSO sets are the smallest over-determined subsets with redundancy which can be obtained.

For diagnosis purposes, it is only necessary to consider the over-determined part $\mathrm{M}^{+}$because faults that appear in any equations in the exactly determined part are not detectable because of the lack of redundancy. In Figure 6.4, the faults $f_{1}, f_{2}$, and $f_{3}$ are not detectable, and the faults $f_{4}, f_{5}$, and $f_{6}$ are in an ideal case detectable. However, measurement noise can, e.g., disturb the test quantities that can result in a fault which is not detectable in a practical application.

\subsubsection{Investigation of Actuator Fault and Health Parameter Isolation}

In Krysander and Frisk (2008), a method is presented where the over-determined $\mathrm{M}^{+}$ part is divided into a number of smaller subsets of equations, i.e., equivalence classes. The advantage with using these subsets is that if any of the equations in the subset is removed, the remaining equations in the subset get exactly determined, thus the degree of redundancy is equal to one. In practice, when a diagnosis test (or residual generator) is constructed it is necessary to have at least one redundant equation. In an equivalence class only one redundant equation is available. When this equation is removed the faults in the remaining subset are no longer detectable. Thus, it is not possible to construct two tests that discriminate between the two (or more) faults in the same equivalence class, and therefore, they are not isolable from each other. A decomposition of the over-determined part in Figure 6.4 is shown in Figure 6.5 where the equivalence classes are determined.

To analyze the isolation performance of the actuator faults and the health parameters in the gas turbine model (6.11) the structural model is studied. The sensor faults are not considered since they appear automatically in the over-determined part and appear also in separate equivalence classes. In the analysis, the derivative of the health parameters is not considered and the deterioration in the components is treated as fault. This is written:

$$
\begin{aligned}
\eta_{i} & =f_{i, \eta}(\ldots)+\Delta \eta_{i} \\
\Gamma_{i} & =f_{i, \Gamma}(\ldots)+\Delta \Gamma_{i} \\
\tilde{u}_{i} & =u_{i}+f_{i}
\end{aligned}
$$

where $f_{i, j}$ represents the look-up tables, $u_{i}$ represents the input signals, and $\Delta \eta_{i}, \Delta \Gamma_{i}$, and $f_{i}$ are all considered to be zero for the healthy gas turbine. In Figure 6.6, the structural model of the gas turbine diagnosis model using the Dulmage-Mendelsohn decomposition is shown. The structural model is divided into the exactly-determined $M^{0}$, and the over-determined $\mathrm{M}^{+}$part. Since only the over-determined part is considered in the FDI-system the first 390 equations can be removed. For example, variables that appear in the exactly-determined part (and can be removed) is, e.g., $\varphi_{i}$ which is the angle of the shaft shown in $(5.24 \mathrm{~b})$. The shaft angles are also state variables. Since all the considered faults and health parameters appear in the over-determined part they are detectable.

In Figure 6.7, the over-determined part from Figure 6.6 is divided into a number of equivalence classes. It is shown that the faults in the input signals of power and speed 


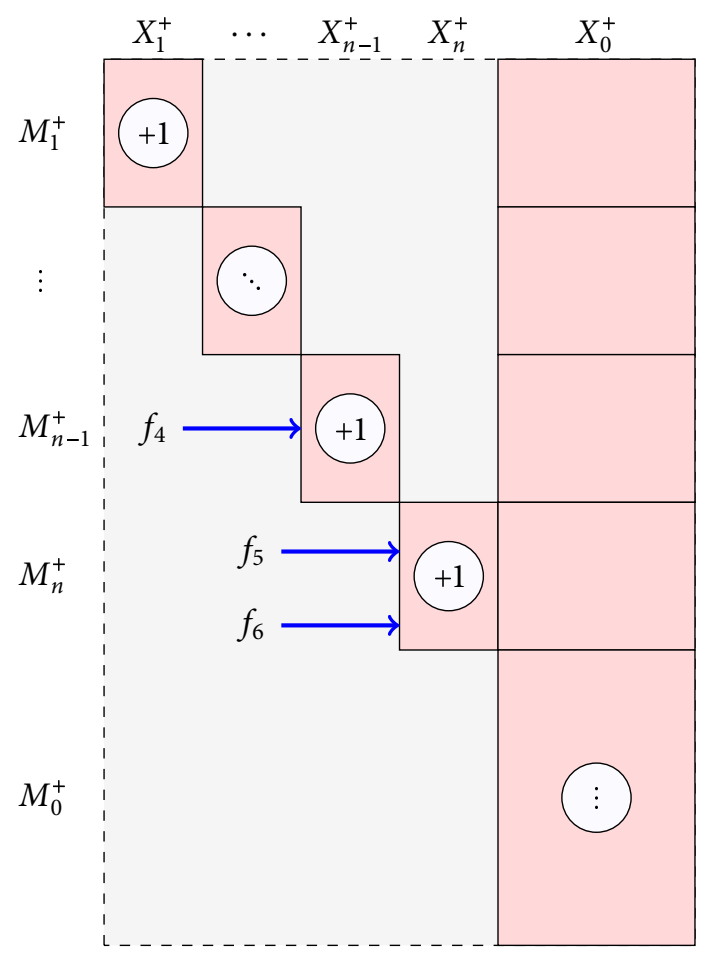

Figure 6.5: In the figure, the decomposition of the over-determined $M^{+}$part from Figure 6.4 into equivalence classes is shown. The equivalence classes are the sets of pair $\left(M_{i}^{+}, X_{i}^{+}\right)$where $i=1 \ldots n$. The equivalence classes have one more equation than the number of variables which results in that the faults $f_{5}$ and $f_{6}$ may be detectable but not isolable from each other. Since $f_{4}$ isn't in the same equivalence class as $f_{5}$ and $f_{6}$, the fault $f_{4}$ may be isolable from fault $f_{5}$ and $f_{6}$. 
of the generator are not isolable. The other fault and health parameters may be isolable depending of, e.g., how the tests are constructed or the noise level in the measurement sensors.

\subsubsection{DAE-index 1 Conservation in the Over-Determined $M^{+}$Part}

In the diagnosis tests, it is only the over-determined part that is of interest since it is only in this part of the model redundancy is available. Therefore, it is of great importance if the over-determined part of the model is a DAE-index 1 system when the index reduced gas turbine model (6.16a) is a DAE-index 1 system. This is true and is shown in Theorem 6.4.1.

Theorem 6.4.1 The over-determined $M^{+}$part of the DAE-index 1 system in (6.15) is also a DAE-index 1 system when the measurement equations are removed.

Proof The index-1 DAE system:

$$
\begin{aligned}
E \dot{x}_{1} & =f\left(x_{1}, x_{2}, u\right) \\
0 & =g\left(x_{1}, x_{2}, u\right) \\
y & =h\left(x_{1}, x_{2}\right)
\end{aligned}
$$

has an invertible and constant matrix $E$, and a non-singular (at least locally) Jacobian matrix $\partial g / \partial x_{2}$, see e.g., Ascher and Petzold (1998). This can be written structurally:

\begin{tabular}{c|cc|c} 
& $\dot{x}_{1}$ & $x_{2}$ & $x_{1}$ \\
\hline$e_{1}:$ & $\times$ & $\times$ & $\times$ \\
$e_{2}:$ & 0 & $\times$ & $\times$ \\
\hline$e_{3}:$ & 0 & $\times$ & $\times$
\end{tabular}

where $e_{1}$ represents the set of dynamic equations, $e_{2}$ represents the set of algebraic equations, and $e_{3}$ represents the set of measurement equations. Since the the matrix $E$ and the Jacobian matrix $\partial g / \partial x_{2}$ are non-singular, a matching between the pair $\left\{\left\{e_{1}\right\},\left\{\dot{x}_{1}\right\}\right\}$ and $\left\{\left\{e_{2}\right\},\left\{x_{2}\right\}\right\}$ exists, which results in a matching between the pair $\left\{\left\{e_{1}, e_{2}\right\},\left\{\dot{x}_{1}, x_{2}\right\}\right\}$ that also exists, independently of the third equation set $e_{3}$.

Next step is to make a Dulmage-Mendelsohn decomposition of the system (where $\dot{x}_{1}$ and $x_{1}$ are lumped together) in (6.18). The rows and columns in the Dulmage-Mendelsohn composition can be permuted to obtain the specific structure:

\begin{tabular}{c|cc|cc} 
& $\dot{x}_{11}$ & $x_{21}$ & $\dot{x}_{12}$ & $x_{22}$ \\
\hline$e_{11}:$ & $\times$ & $\times$ & $\times$ & $\times$ \\
$e_{12}:$ & 0 & $\times$ & $\times$ & $\times$ \\
\hline$e_{21}:$ & 0 & 0 & $\times$ & $\times$ \\
$e_{22}:$ & 0 & 0 & 0 & $\times$ \\
\hline$e_{3}:$ & 0 & 0 & 0 & $\times$
\end{tabular}

where the variables $x_{1}$ and $x_{2}$ are split into two parts:

$$
\begin{aligned}
& x_{1}=\left\{x_{11}, x_{12}\right\} \\
& x_{2}=\left\{x_{21}, x_{22}\right\}
\end{aligned}
$$




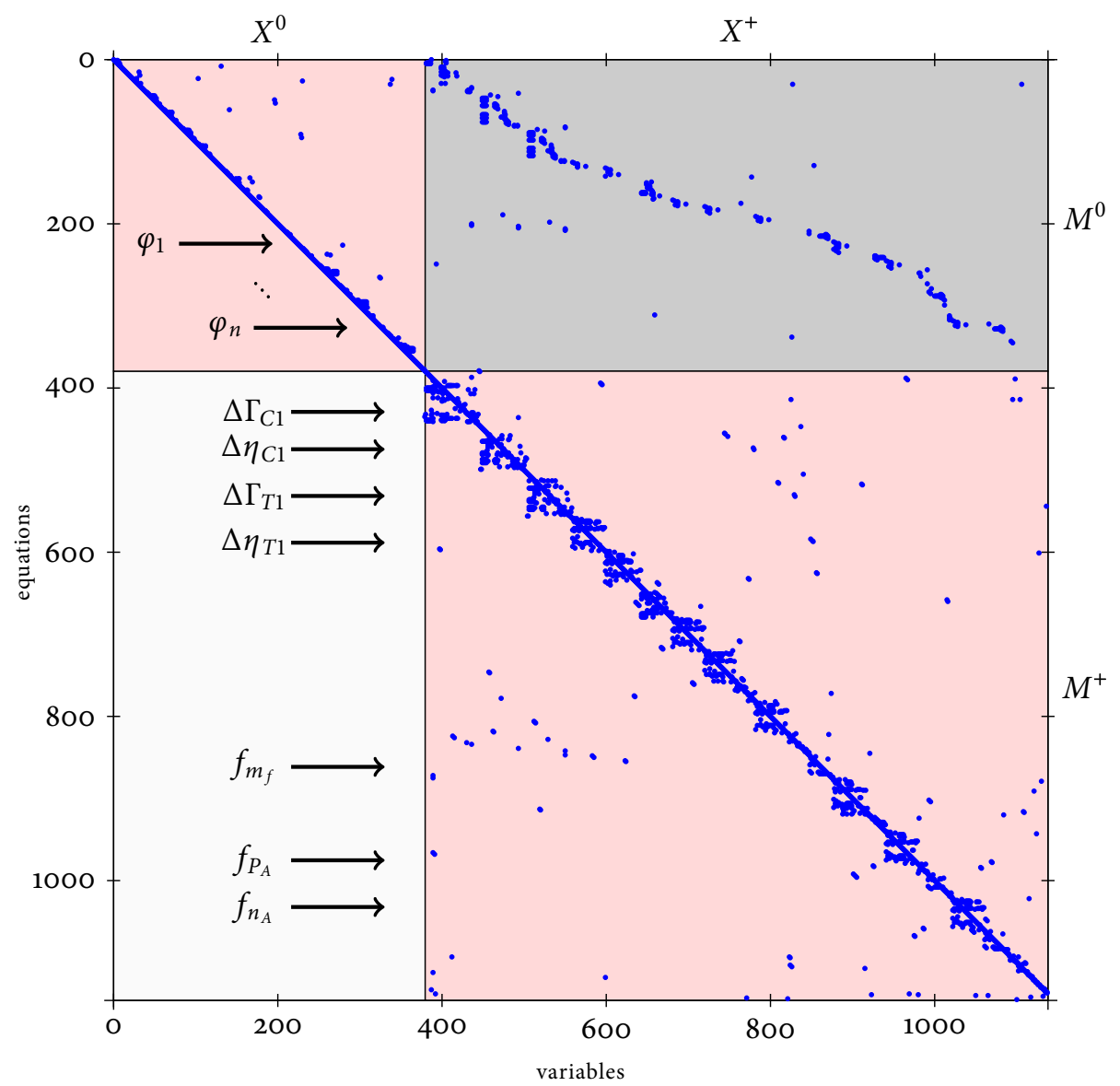

Figure 6.6: In the figure, the structural model of the gas turbine diagnosis model using the Dulmage-Mendelsohn decomposition is shown. The equations where the actuator faults and health parameters are injected are shown with arrows in the figure. The structural model is divided into two parts, where the first part is the exactly-determined $M^{0}$ part and consists of the first 390 equations. The second part is the over-determined $M^{+}$part and consists of the remaining equations. It is not necessary to considered the $M^{0}$ part for diagnosis purposes since the absence of redundancy and this part can be removed in the diagnosis tests. Since all the considered faults and health parameters appear in the over-determined part they are detectable. Variables that appear in the exactly-determined part is, e.g., $\varphi_{i}$ which is the angle of the shaft from (5.24b). 


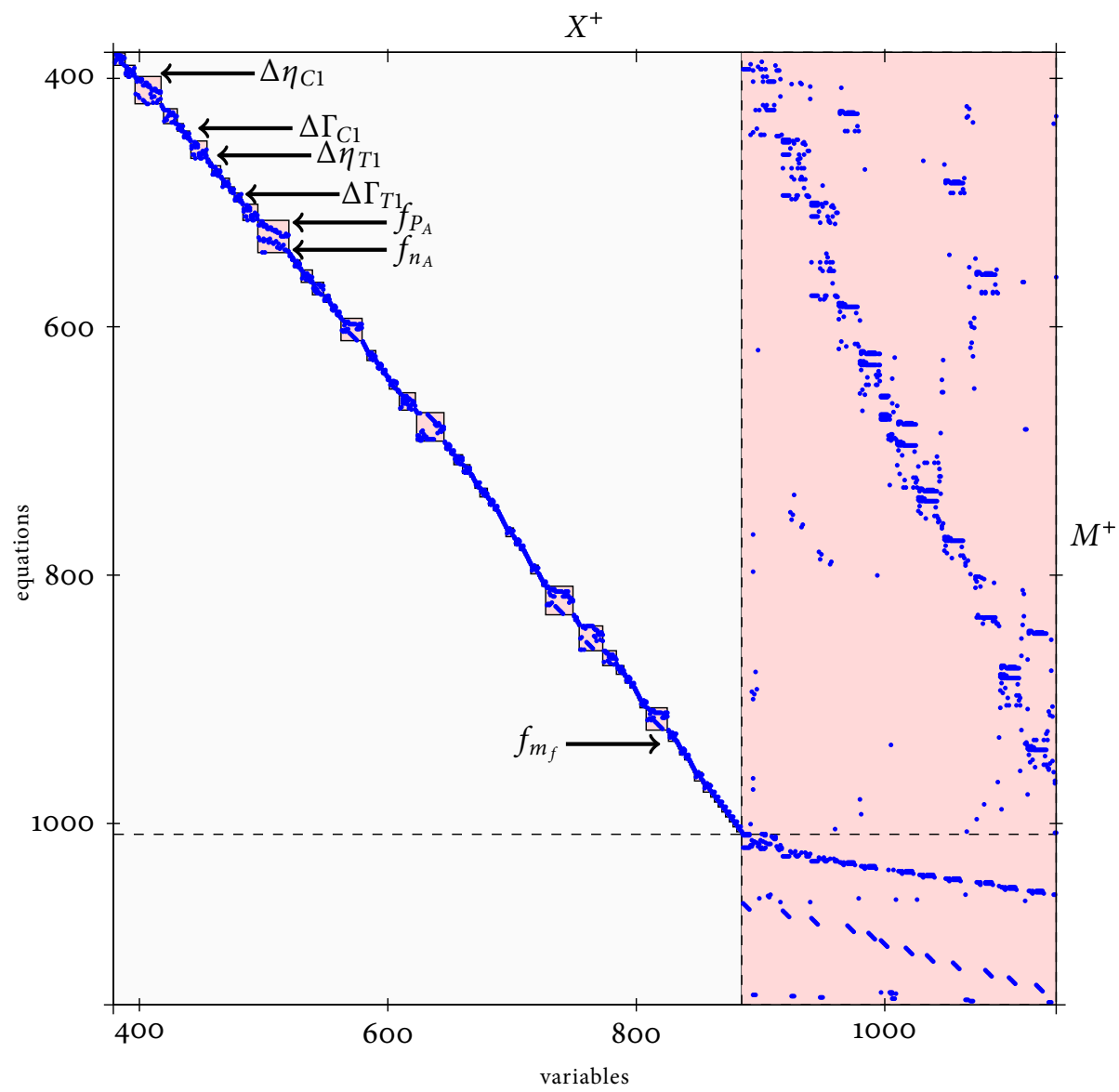

Figure 6.7: In the figure, the over-determined part of the structural model in Figure 6.6 is divided into a number of equivalence classes. The equations where the actuator faults and health parameters are injected are shown with arrows in the figure. Since faults $f_{P_{A}}$ and $f_{n_{A}}$ appear in the same equivalence class they are not isolable. All the other fault modes may be isolable since they appear in different equivalence classes. Since many (eight) sensors are used, the equivalence classes get small. For a smaller number of sensors, the equivalence classes get larger. 
The equation set $\left\{e_{21}, e_{22}, e_{3}\right\}$ in (6.20) is a redundant set while the equation set $\left\{e_{11}, e_{12}\right\}$ has no redundancy. Since the original system (6.19) has a matching in $\left\{\left\{e_{1}, e_{2}\right\},\left\{\dot{x}_{1}, x_{2}\right\}\right\}$ and matching of variables and equations is not affected by row and column permutations, also $\left\{\left\{e_{11}, e_{12}, e_{21}, e_{22}\right\},\left\{\dot{x}_{11}, x_{21}, \dot{x}_{12}, x_{22}\right\}\right\}$ has a matching. Since the third quadrant has only zeros, a matching in the forth quadrant must exist. Therefore, it is possible to find a matching for the pair $\left\{\left\{e_{21}, e_{22}\right\},\left\{\dot{x}_{12}, x_{22}\right\}\right\}$ and the sub-system is an index-1 DAE.

Remark 6.4.2 A matching between the pair $\left\{\left\{e_{i}\right\},\left\{x_{i}\right\}\right\}$ means that it can be possible to calculated the variable $x_{i}$ using the equation set $e_{i}$. If a matching does not exist, the unknown variables $x_{i}$ cannot be calculated using the equation set $e_{i}$. In a redundant equation set, unknown variables can be matched in several ways.

\subsubsection{Diagnosis Test Equation}

The diagnosis test equations are chosen using the Dulmage-Mendelsohn decomposition of the index reduced diagnosis model (6.15). The over-determined $M^{+}$part of the system (6.15) is:

$$
\begin{aligned}
E \dot{x}_{1} & =\tilde{f}\left(x_{1}, x_{2}, u\right) \\
0 & =\tilde{g}\left(x_{1}, x_{2}, u\right) \\
y & =\tilde{h}\left(x_{1}, x_{2}\right)
\end{aligned}
$$

where the algebraic constraints can be written, at least locally, $x_{2}=G\left(x_{1}, u\right)$ since the system is in an index-1 DAE form. The state space form is obtained through an elimination of the algebraic variable $x_{2}$ and an inversion of the matrix $E$ :

$$
\begin{aligned}
\dot{x}_{1} & =f\left(x_{1}, u\right) \\
y & =h\left(x_{1}\right)
\end{aligned}
$$

where $x_{1}$ represents the state variables, $u$ represents the input signals, and $y$ represents the measurement signals. Before the observers are constructed in the Section 6.6, the observability of the model is necessary to check.

\subsection{Observability Analysis}

If observer based techniques should be considered when the diagnosis tests are designed, it is necessary that information about the state of the system is available in the measurements, i.e., the system should be observable. It is strictly speaking not necessary that the system is required to be observable. An observability requirement could be replaced by a detectability requirement, i.e., any non-observable modes are stable, but here observability will be analyzed.

An observability analysis of the index reduced diagnosis model (6.16) shows that unobservable modes are present. To determine if a nonlinear system is observable is 
in general a difficult question (Hermann and Krener, 1977). A number of different observability criteria is available in, e.g., Nijmeijer and Fossen (1999). A starting-point is to check if the nonlinear system is locally observable through a linearization in a suitable operating point $\left(x_{0}, u_{0}\right)$ of the system in $(6.22)$. The system matrices are:

$$
A=\left.\frac{d f}{d x}\right|_{\left(x_{0}, u_{0}\right)}, B=\left.\frac{d f}{d u}\right|_{\left(x_{0}, u_{0}\right)}, C=\left.\frac{d h}{d x}\right|_{\left(x_{0}, u_{0}\right)} .
$$

The linearized system, near the point $\left(x_{0}, u_{0}\right)$, is written:

$$
\begin{aligned}
& \dot{x}=A\left(x-x_{0}\right)+B\left(u-u_{0}\right) \\
& y=C\left(x-x_{0}\right)+y_{0}
\end{aligned}
$$

For the linear system in (6.24), the observability can be checked according to the observability matrix $\mathbb{O}(A, C)$ in Theorem 6.5.1.

Theorem 6.5.1 A pair $A \in \mathbb{R}^{n \times n}$, and $C \in \mathbb{R}^{m \times n}$ is observable if and only if the observability matrix

$$
\mathbb{O}(A, C)=\left[\begin{array}{c}
C \\
C A \\
\vdots \\
C A^{p-1}
\end{array}\right]
$$

has full rank $n$, for a $p \leq n$.

Proof See, e.g., Kailath (1980).

\subsubsection{Structural Observability}

To handle numerical problems, the structural observability presented in Shields and Pearson (1976) is investigated. Since the structure of the model is used, the method can only provides a necessary condition for the observability. Since the model is physical based, it can be assumed that structural observability implies observability. The structural observability criteria can be stated:

Theorem 6.5.2 A pair $A \in \mathbb{R}^{n \times n}$, and $C \in \mathbb{R}^{m \times n}$ is structural observable if and only if the generalized observability matrix

$$
\mathbb{O}_{s}(A, C)=\left[\begin{array}{cccccc}
I & A & 0 & \ldots & 0 & 0 \\
0 & I & A & \ldots & 0 & 0 \\
\vdots & \vdots & \vdots & & \vdots & \vdots \\
0 & 0 & 0 & \ldots & I & A \\
0 & 0 & 0 & \ldots & 0 & C \\
0 & 0 & 0 & \ldots & C & 0 \\
\vdots & \vdots & \vdots & & \vdots & \vdots \\
0 & C & 0 & \ldots & 0 & 0 \\
C & 0 & 0 & \ldots & 0 & 0
\end{array}\right]
$$

with dimension $\left[n^{2}+n(m-1)\right] \times n^{2}$ has structural rank $n^{2}$. 
Proof See Shields and Pearson (1976).

The structural rank of a matrix can easily be checked using graph theoretical algorithms for matching in bi-partite graphs, e.g., the Dulmage-Mendelsohn decomposition. In Matlab, the sprank command can be used.

\subsubsection{Removing of Unobservable Modes}

For the gas turbine diagnosis model, the linearization of the system in (6.16) has unobservable modes, which can be checked using the structural observability method. Thus, if an observer should be constructed, these unobservable modes need to be removed. As a consequence, when the exactly-determined part $M^{0}$ is removed, a number of unobservable modes also disappear, which is stated in Theorem 6.5.3.

Theorem 6.5.3 Consider the semi-explicit index-1 DAE system (6.15). A state variable that appears in the exactly-determined $M^{0}$ part of the structural model is unobservable.

Proof According to Theorem 6.4.1, the semi-explicit index-1 DAE system can be written structurally:

\begin{tabular}{c|cc|cc|cc} 
& $\dot{x}_{11}$ & $x_{21}$ & $\dot{x}_{12}$ & $x_{22}$ & $x_{11}$ & $x_{12}$ \\
\hline$e_{11}:$ & $\times$ & $\times$ & $\times$ & $\times$ & $\times$ & $\times$ \\
$e_{12}:$ & 0 & $\times$ & $\times$ & $\times$ & $\times$ & $\times$ \\
\hline$e_{21}:$ & 0 & 0 & $\times$ & $\times$ & 0 & $\times$ \\
$e_{22}:$ & 0 & 0 & 0 & $\times$ & 0 & $\times$ \\
\hline$e_{3}:$ & 0 & 0 & 0 & $\times$ & 0 & $\times$
\end{tabular}

where $x_{1}=\left\{x_{11}, x_{12}\right\}$, and $x_{2}=\left\{x_{21}, x_{22}\right\}$. In (6.25) a Dulmage-Mendelsohn decomposition of the DAE system (where $\dot{x}_{1}$ and $x_{1}$ are lumped) is performed together with an extra rearrangement of the rows and the columns to obtain the specific structure. According to Theorem 6.4.1, a matching of the pair $\left\{\left\{e_{11}, e_{12}\right\},\left\{\dot{x}_{11}, x_{21}\right\}\right\}$, and the pair $\left\{\left\{e_{21}, e_{22}\right\},\left\{\dot{x}_{12}, x_{22}\right\}\right\}$ exists. The system in (6.25) can be written analytically:

$$
\begin{aligned}
\dot{x}_{11} & =f_{1}\left(x_{21}, \dot{x}_{12}, x_{22}, x_{11}, x_{12}\right) \\
x_{21} & =g_{1}\left(\dot{x}_{12}, x_{22}, x_{11}, x_{12}\right) \\
\dot{x}_{12} & =f_{2}\left(x_{22}, x_{12}\right) \\
x_{22} & =g_{1}\left(x_{12}\right) \\
y & =h_{1}\left(x_{22}, x_{12}\right)
\end{aligned}
$$

Substitution of the algebraic constraints (6.26b) and (6.26d) into dynamic equations (6.26a) and (6.26c) gives:

$$
\begin{aligned}
\dot{x}_{11} & =\tilde{f}_{1}\left(x_{11}, x_{12}\right) \\
\dot{x}_{12} & =\tilde{f}_{2}\left(x_{12}\right) \\
y & =h_{2}\left(x_{12}\right)
\end{aligned}
$$


System in (6.27) is linearized to give the matrices $A$ and $C$ :

$$
A=\left(\begin{array}{cc}
x_{11} & x_{12} \\
\times & \times \\
0 & \times
\end{array}\right) \text {, and } C=\left(\begin{array}{cc}
x_{11} & x_{12} \\
0 & \times
\end{array}\right)
$$

which gives:

$$
C A^{k}=\left(\begin{array}{ll}
0 & \times
\end{array}\right)
$$

for $k \geq 0$. The observability matrix $\mathbb{O}$ in Theorem 6.5.1 cannot have full rank, so differentiated states that appear in the exactly-determined part are not observable.

When the $\mathrm{M}^{+}$part is considered, unobservable modes which appear in the exactlydetermined part are removed according to Theorem 6.5.3. It is verified that the linearization of (6.22) is structurally observable, which indicates that observer based techniques can be considered when the test quantities are constructed. The unobservable modes which are removed are the angle variables $\varphi_{i}$. When these state variables are removed, the algebraic constraints $\tilde{g}_{0}$ in $(6.14 \mathrm{~b})$ can also be removed since they consist of only angle dependencies. The angle variables were also shown in Figure 6.6.

\subsubsection{Number of Health Parameters in the Model}

An important question, when an diagnosis test is constructed, is how many health parameters that can be introduced in the model. The number of health parameters affect the observability of the model and the maximum number is equal to the number of unique measurement sensors. This necessary condition is summarized in Lemma 6.5.4. Where in the model the health parameters appear may also affect the observability.

Lemma 6.5.4 A pair $A \in \mathbb{R}^{n \times n}$, and $C \in \mathbb{R}^{m \times n}$ is not observable if the number of health parameters is larger then the number of measured states $n_{c}$.

Proof Let $x \in \mathbb{R}^{n_{x}}, h \in \mathbb{R}^{n_{h}}$, and the number of measured states $n_{c}$. The structural rank of a matrix can never be smaller if nonzero elements are added to the element positions of the matrix. This gives the opportunity to investigate the structural rank of the last column in the matrix below:

$$
\tilde{\mathbb{O}}_{\mathrm{s}}=\left[\begin{array}{cccc}
\vdots & & \vdots & \vdots \\
\times & \ldots & \times & 0 \\
\times & \ldots & \times & A \\
\times & \ldots & \times & C \\
\times & \ldots & \times & 0 \\
\vdots & & \vdots & \vdots
\end{array}\right]
$$

Assume that all element positions in the matrix pair $(A, C)$, except for the health parameter states, are occupied according to:

$$
A=\left(\begin{array}{cl}
x & h \\
\times & \times \\
0 & 0
\end{array}\right) \text {, and } C=\left(\begin{array}{cl}
x & h \\
\times & 0
\end{array}\right)
$$


where $A \in \mathbb{R}^{n \times n}, C \in \mathbb{R}^{n_{c} \times n}$, and $n=n_{x}+n_{h}$. If the structural rank of $\tilde{\mathbb{O}}_{s}$ should be $n^{2}$, the last $n$ columns need to have a structural rank $n$. Further, the last $n$ columns can be written:

$$
\left[\begin{array}{l}
A \\
C
\end{array}\right]=\left[\begin{array}{ll}
\times & \times \\
0 & 0 \\
\times & 0
\end{array}\right]
$$

where the zeros are omitted, and has the structural rank $n_{x}+n_{c}$. The pair $(A, C)$ is not observable if

$$
n_{x}+n_{c}<n
$$

which can be written:

$$
n_{c}<n_{h}
$$

where $n_{x}=n-n_{h}$ is used. This gives the matrix pair $(A, C)$ is not observable if $n_{c}<n_{h}$.

Remark 6.5.5 The same arguments, as in Lemma 6.5.4, can be considered to verify that two health parameters that appear in the same equivalence class in Figure 6.5 are not observable.

\subsection{Filter Design}

A common approach in the gas turbine diagnosis literature to estimate health deterioration is to use tests based on observers. The observers are either linear (Volponi, 2003; Luppold et al., 1989) or nonlinear (Borguet and Léonard, 2008; Dewallef et al., 2006; Rausch et al., 2007) Kalman filters. In these papers, the application is for in-flight monitoring purposes but the focus in the present work is on industrial gas turbines. For stationary gas turbines, the calculating capacity is assumed to be larger then for an in-flight monitoring case. Thus, more sophisticated filters can be considered for industrial gas turbines, and the main objective here is to design a nonlinear filter in the form:

$$
\begin{aligned}
& \dot{\hat{x}}=f(\hat{x}, u)+K(y-\hat{y}) \\
& \hat{y}=h(\hat{x})
\end{aligned}
$$

where $f$ and $h$ are functions, $u$ represents the input signals, $y$ represents the measurement signals, and $K$ is the observer gain which can be considered as a design parameter. The estimation error, or the residual, $r=y-\hat{y}$ is amplified through the $K$ matrix. Thus, if the observer gain $K$ is large, the observer relies more on the measurements than on the model equations, and if the observer gain $K$ is small the observer relies more on the model equations than on the measurements. This means that a large $K$ results in state estimates which is more sensitive to measurement noise than if a small $K$ is used. On the other hand, a small $K$ corresponds to estimation errors where model uncertainties get more significant. Thus, when the observer is designed, it is possible to tune between a fast and noisy and a slow and filtered estimator. 
A special type of a nonlinear Kalman filter are the so-called Constant Gain Extended Kalman Filter (CGEKF), presented in Safonov and Athans (1978), where also the robustness and stability of the filter concept are investigated. The CGEKF is a simplification of the Extended Kalman Filter (EKF) Kailath et al. (2000). In the CGEKF, the observer gain is calculated in advance for a given operating point and can be calculated offline which is not the case for an EKF. Thus, the computational burden is smaller for a CGEKF than for an EKF since the Jacobian of the system (6.30) is not calculated in each time step. In the gas turbine application papers Kobayashi et al. (2005); Sugiyama (2000) the CGEKF methodology is considered. In these papers it is demonstrated that the CGEKF can be used in a wide operating range for an in-flight aircraft engine diagnosis application. These studies indicate that it is not necessary to calculate a new Kalman gain for each operational point during a flight program.

In the present gas turbine application, the CGEKF methodology is investigated since the observer has an attractive form which is suitable for real time application for a good choice of the operating point. Usually works a CGEKF well for a suitable choice of the operating point where the observer gain is calculated. The advantage is that the observer gain can be determined off-line which is attractive for an estimator that is used in a real time application.

The starting point of the observer construction methodology is the gas turbine diagnosis DAE model defined in (6.21). How the equations, used in the filters, are selected is shown in Section 6.4. Before a summary of the filter design procedure is presented, the equations of the linear/nonlinear Kalman observers are shown and important steps such as observability and observer tuning are discussed.

\subsubsection{Kalman Filter}

A linear dynamic system, expressed in state space form, can be written:

$$
\begin{aligned}
& \dot{x}=A x+B u+G w \\
& y=C x+D u+v
\end{aligned}
$$

where $A \in \mathbb{R}^{n_{x} \times n_{x}}, B \in \mathbb{R}^{n_{x} \times n_{u}}, C \in \mathbb{R}^{n_{y} \times n_{x}}$, and $D \in \mathbb{R}^{n_{y} \times n_{u}}$ are system matrices, $G \in$ $\mathbb{R}^{n_{x} \times n_{w}}$ is a gain matrix of the process noise, $u$ is the input signal, $w$ is white process noise, and $v$ is white measurement noise. The noise signals satisfy:

$$
\begin{gathered}
E\left[w(t) w(s)^{T}\right]=Q \delta(t-s), \quad E\left[v(t) v(s)^{T}\right]=R \delta(t-s), \\
E[w(t)]=E[w(s)]=0, \quad E[v(t)]=E[v(s)]=0,
\end{gathered}
$$

for the dummy scalar variables $t, s$ and the Dirac function $\delta$. The Kalman filter equations (Kailath et al., 2000) of system in (6.31), are:

$$
\begin{aligned}
& \dot{\hat{x}}=A \hat{x}+B u+K(y-C \hat{x}-D u) \\
& \hat{y}=C \hat{x}+D u+v
\end{aligned}
$$

The Kalman gain $K$ is:

$$
K=P C^{T} R^{-1}
$$


where $P$ is the solution to the Riccati equation

$$
\dot{P}=A P+P A^{T}+G Q G^{T}-P C^{T} R^{-1} C P+Q
$$

for the given noise matrices $Q$ and $R$, and for a given initial probability condition $P(0)=E\left[(x(0)-\hat{x}(0))(x(0)-\hat{x}(0))^{T}\right]$.

\subsubsection{Stationary Kalman Filters}

The stationary Kalman filter is obtained by setting $\dot{P}=0$ in (6.35).

\subsubsection{Nonlinear Kalman Filters}

A nonlinear dynamic system can be written in the form:

$$
\begin{aligned}
\dot{x} & =f(x, u, w) \\
y & =g(x, u)+v \\
w & \sim(0, Q) \\
v & \sim(0, R)
\end{aligned}
$$

where the linearization

$$
A=\left.\frac{\partial f}{\partial x}\right|_{\hat{x}, u}, C=\left.\frac{\partial g}{\partial x}\right|_{\hat{x}, u}, G=\left.\frac{\partial f}{\partial w}\right|_{\hat{x}, u}
$$

is applied when the Kalman gain $K$ is calculated according to the Riccati equation in (6.34). Then it is possible to construct the two nonlinear Kalman filters EKF and CGEKF. These filters can be written:

1. Extended Kalman Filter (EKF):

$$
\begin{aligned}
& \dot{\hat{x}}=f(\hat{x}, u)+K(\hat{x}, u)(y-h(\hat{x}, u)) \\
& \hat{y}=g(\hat{x}, u)
\end{aligned}
$$

where the Kalman gain $K(\hat{x}, u)$ is updated in every time step according to (6.34).

2. Constant Gain Extended Kalman Filter (CGEKF):

$$
\begin{aligned}
& \dot{\hat{x}}=f(\hat{x}, u)+K(y-h(\hat{x}, u)) \\
& \hat{y}=g(\hat{x}, u)
\end{aligned}
$$

where the Kalman gain $K$ is calculated for the stationary Kalman filter in one operational point according to (6.34). 


\subsection{Parsers for an Automatic Extraction of Sub Systems}

Since the number of variables and equations in the gas turbine model is large, a systematic method to analyze and select relevant equations automatically is attractive to have. The number of equations and variables is more than 1000 as shown in the structural model in Figure 6.6, which is too large for manual processing. It should also be easy to investigate various types of diagnosis setups such as models with different number of sensors and health parameters. Those analyses are difficult to perform in the Dymola environment, thus a parser for model exportation is necessary to have.

To analyze model properties, the Matlab environment is preferably used since it has available routines for, e.g., structural methods, model linearization, and Kalman gain calculation. Structural methods are, e.g., the Dulmage-Mendelsohn decomposition (dmperm.m), and the equivalent class construction. Another motive to use Matlab is that filtering and other signal manipulations are easier to make than in the Dymola environment. The final aim with the parsers is to transform the flat Modelica gas turbine model to a number of test quantities based on Kalman filters. These test quantities have the form shown in (6.30) where $f$ and $g$ are Matlab m-functions.

In this section, parsers used for: (i) DAE model extraction, (ii) structural model construction, (iii) DAE-index reduction, and (iv) state space form construction are presented. The name parser is used since most of the time there are text strings that are manipulated. For the equation manipulation, the MuPAD symbolic engine is called from the implemented parsers. The MuPAD symbolic engine is a part of the Symbolic Math Toolbox contained in Matlab. In this work, Matlab version 7.11 and MuPAD version 5.5 are used.

\subsubsection{Automatic Extraction of the DAE Model}

To fulfill the requirement, a parser that can export the diagnosis model implemented in Modelica to the Matlab environment is developed. The parser is relatively simple and can only handle a restricted functionality but the functionality is sufficient to be used together with models constructed in the GTLib package. After the model is exported, the MuPAD symbolic engine is invoked to handle the symbolic transformation of the equations and variables according to the methods described in this section. A brief description of the DAE extraction parser is summarized in:

1. Before any symbolic transformation of the model is done in Dymola, it is possible to save the flat Modelica model. The flat Dymola model is a text file that is called .mof, and contains information, such as equations, variables, parameters, constants, functions, etc. All this information is needed when the model is simulated outside the Dymola environment. In the .mof-file, all the object oriented hierarchies are removed, so the file consists of pure equations and variables. Hence, the .mof-file is the input to the DAE extraction parser.

2. All functions that are called from the Dymola model appear in the beginning of the .mof-file. These functions are for example the NASA polynomials that describe the gas properties in a specific gas, and the functions that are implemented in 
GTLib which are mentioned in subsection 5.3.3. All of these functions are removed from the .mof-file and converted to Matlab functions for future investigations.

3. Next step is to find, save, and remove variables, parameters and constants from the file. The values of the parameters, the constants, and the initial values of the variables are saved. The variables appear with different declarations such as Real, Integer, and Boolean which are handled separately. These variables can also appear as the type input and output which also have to be handled separately.

4. Equations that consist of if -statements have to be treated with care, since the global equation system changes with the if-condition. For example, a model with six if-statements (that doesn't has any elseif part) gives $2^{6}=64$ different equation systems. This can be difficult to handle in practice, so here it is necessary to state the condition of the if-statements. Fortunately, most of the if-statements in the model come from the flow direction of the gas. Thus, it is assumed that the direction is known, which transforms respective if-statement to only one equation.

5. Now the . mof-file consists of only pure equations, and the final step is to interpret these equations to a format that is familiar by the MuPAD symbolic engine. The earlier saved variables, parameters, and constants have also to be converted to a format that the MuPAD symbolic engine can understand.

The output of the DAE extraction parser is a differential algebraic equation system with attached variables, and given input, and output signals. The output DAE system can be treated by the MuPAD symbolic engine available in the Matlab environment.

\subsubsection{Structural Model Parser}

The structural model of the DAE model can easily be acquired through a variable find approach by the MuPAD symbolic engine. The acquired DAE system in the previous step consists of a number of functions. These functions can be look up tables, algorithms, and mathematical functions. In the structural model, it is not necessary to consider the internal variables in each function. Therefore, it is sufficient to only consider the input and the output variables for each function. The input and the output signals in the analytical model, as can be seen in Figure 6.2, are not considered in the structural model since they are known quantities.

The output of the structural model parser is the structural model of the DAE model that is given as an input argument to the parser.

\subsubsection{Index Reduction Parser}

The structural model together with the DAE model are inputs to the index reduction parser. Actual rows in the equation system that are needed to be differentiated are determined with Pantelides algorithm (Pantelides, 1988). These rows are differentiated, and the underlying equations are saved to get proper initial conditions to the differentiated equation system. 
The output from the Index Reduction Parser is an equation system that has a differential algebraic index equal to 1 . The system is also transformed into a form that the $E$-matrix in (6.15) has full rank according to the variable elimination procedure described in Section 6.3.1.

\subsubsection{State Space Form Construction Parser}

The intention with this parser is to easily construct a set of runnable Matlab functions that can be used to simulate the index reduced equation system defined previously. For the parser, it is assumed that the input equation system is an index-1 DAE (which has no redundancy), and can be written in a semi-explicit form. The overall Dulmage-Mendelsohn decomposition of the system is shown in Figure 6.8, where the set of equations and vari-

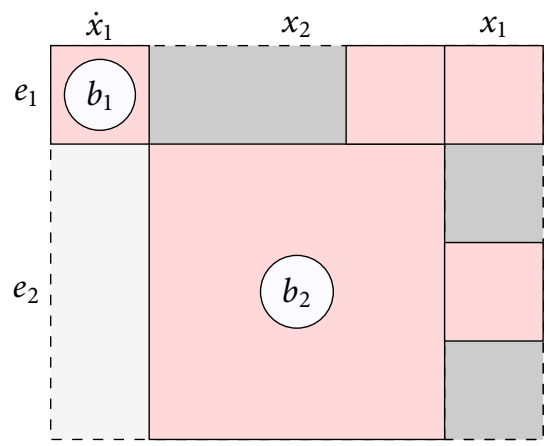

Figure 6.8: Dulmage-Mendelsohn decomposition of a system in semi-explicit form where $\dot{x}_{1}$ and $x_{2}$ are considered as unknown variables. In each step, when the system is solved, $x_{1}$ is the previous state of the system and hence it can be considered as an input signal.

ables $\left\{e_{1}, \dot{x}_{1}\right\}$ is the differential part, and $\left\{e_{2}, x_{2}\right\}$ is the algebraic part of the system. This decomposition is done because in each time step for the solver, $x_{1}$ is the previous state of the system and hence it can be seen as an input signal to the differential part $b_{1}$.

For the algebraic part $b_{2}$ in Figure 6.8, the Dulmage-Mendelsohn is once again utilized, and gives the results shown in Figure 6.9. To solve the overall system, variable $\tilde{x}_{n}$ is first calculated. This result is utilized in the next equation set $\tilde{e}_{n-1}$ and $\tilde{x}_{n-1}$ is then calculated. This solving process goes on until the last variable $\tilde{x}_{1}$ is calculated. If the set $\tilde{e}_{i}$ consists of two or more equations, it is said that $c_{i}$ is a strongly connected component. This means that the variables contained in $c_{i}$ cannot be substituted without transformation, i.e., an inversion of the component is necessary. For linear systems, this can be done easily since Gaussian elimination can be applied to obtain a substitution chain, similar to the structural model. For a nonlinear strongly connected component, a numerical solver can preferably be used since an analytic solution can be difficult to find. Another interpretation of the behavior of strongly connected components is to consider a bi-partite graph, which consists of loops or cycles, see, e.g., Blanke et al. (2003). A 


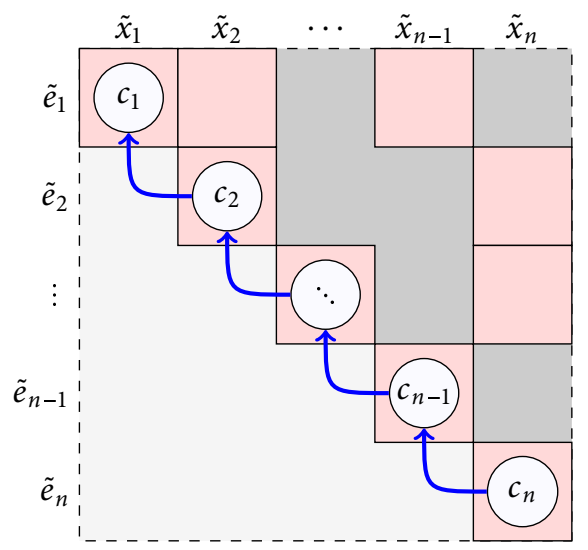

Figure 6.9: In the figure, the Dulmage-Mendelsohn decomposition of the pure algebraic component $b_{2}$ from Figure 6.8 is shown. The arrows in the figure illustrate the solution path.

description of the state space form construction parser is summarized in the pseudo algorithm below:

1. First, the equation system is divided in two parts as illustrated in Figure 6.8. The $b_{1}$ and $b_{2}$ components are located through a DM decomposition, where the sets of unknown variables are $\dot{x}_{1}$ and $x_{2}$. The variable $x_{1}$ is considered as known since the previous state of the system is known.

2. The algebraic part $b_{2}$ is treated separately, and the structural model is considered when the substitution chain is determined as Figure 6.9 illustrates.

3. For each component $c_{i}$, the parser determines if the component consists of only one equation. If this is true, the MuPAD symbolic engine is called and tries to find an analytic solution, i.e., a pure substitution.

4. If the MuPAD symbolic engine failed to find an analytic solution, or if the component is strongly connected, a nonlinear Matlab solver is incorporated in the generated file.

5. After the variables in $x_{2}$ are calculated, they are inserted in the differential part of the system, together with the previous state $x_{1}$. Finally, the state of the system in the next time step can be calculated with a numerical ODE-solver after the component $b_{1}$ in Figure 6.8 is inverted.

The outputs from the parser are the two .m-files $f$ and $G$ that can be interpreted as:

$$
\begin{aligned}
x_{2, n-1} & =\mathrm{G}\left(x_{1, n-1}, x_{2, n-1}^{*}\right) \\
\dot{x}_{1} & =\mathrm{f}\left(x_{1, n-1}, x_{2, n-1}\right)
\end{aligned}
$$


where index $n$ represents the actual time step, and (*) represents an initial guess of the iteration variable (i.e., the previous value of the iteration variable). Input argument $x_{2,0}$ in (6.39a) is needed by the nonlinear solver to get an appropriate starting point. Expression (6.39b) can then be called by an ordinary differential equation solver in Matlab.

\subsection{Conclusion}

In the chapter, a gas turbine diagnosis model is presented that can be used when a FDI-system is designed. In the diagnosis model, faults and estimation parameters used for diagnosis purposes are introduced. The faults are introduced in the actuator and sensor signals, and the estimation parameters are called health parameters which should compensate for gradual deviation in performance.

The diagnosis model is analyzed for: (i) DAE-index, (ii) observability, and (iii) structural properties. The test equations which are used in the FDI-system are chosen using structural methods. Here, the whole over-determined part is chosen. The observability analysis shows that unobservable state variables are removed using the selected set of equations. The split into equivalence classes shows that faults in the input signals of power and speed of the applied generator is not isolable. All the other fault modes and health parameters may be detectable and isolable. To make the analyses, a number of parsers is developed. These parsers take the Modelica gas turbine diagnosis model and convert it into runnable Matlab code. 



\section{Part III}

Application of Methodology 



\section{Chapter 7}

\section{Performance Estimation in Industrial Gas Turbine Engines}

The performance of an industrial gas turbine degrade gradually due to certain factors such as environment air pollution, fuel content, and ageing to mention some of the degradation factors. Degradation due to compressor fouling can partially be restored by an online/offline compressor wash. Therefore, it is important to supervise the degradation to efficiently plan service and maintenance. The gas turbine fleet consists of a lot of individuals, with different kinds of properties and configurations, that have to be monitored by the service engineers. Therefore, it is desirable that it should be easy to construct and evaluate test quantities and collect them in the FDI-system. When the diagnosis test quantities are constructed from a component based model, it should be easy to, e.g., replace components or change parameter values depending of the supervised engine. The main objective of this chapter is to apply the methodology for diagnosis test quantity design presented in Chapter 6 . The diagnosis tests are constructed in an automatic manner directly from the gas turbine diagnosis model. The diagnosis tests can then be used for performance estimation, or as smaller part in a more general FDI-system.

The chapter starts with an introductory evaluation where three basic methods to detect compressor fouling is presented. These methods involve only the measurement signals and not the gas turbine model. In Section 7.3, the well known Measurement Delta method is investigated using the experimental data where the focus is to monitor trends in the deltas. The deltas is generally the difference between a simulated and a measured gas path quantity. In Section 7.4, the nonlinear Kalman filter is designed and generated for the SGT-70o gas turbine model. The filter is then investigated in Section 7.5 where three case studies are presented. In the first case study, simulated data from the reference platform is evaluated for different operational points and different atmospheric weather conditions. In the remaining case studies, experimental data is evaluated. In evaluation 2, the focus is on state estimation for control, where the flame temperature is supervised 
and compared for two filters. In evaluation 3 , the supervision is presented according to detection of a fouled compressor.

\subsection{Background}

Estimation of unmeasured gas turbine performance parameters can be used in, at least, two contexts: (i) monitoring, and (ii) control. In monitoring applications, a typical objective is to study deteriorations in gas turbine components, e.g., compressors and turbines, which affect the estimated performance parameters. Therefore, in monitoring applications it is of great importance to study the deviation from a nominal baseline to decide whether the engine indicates a faulty behavior or not using the gas path measurements. Monitoring of gas turbines, and especially aircraft engines, is a widely studied topic in the gas turbine diagnosis literature (Volponi, 2014; Luppold et al., 1989; Kobayashi and Simon, 2005; Doel, 2003). For control applications, model based feedback control strategies are studied in Watts et al. (1992); Härefors (1997). In these papers, the control strategies are based on linear models which are derived from a thermodynamic engine model. In the controller design, the component deteriorations are not considered which can result in an unnecessary overall reduction in the gas turbine performance. An important signal, used in the controller, is the combustion flame temperature. Since this signal is not measured, the signal needs to be estimated, e.g., by using a model and thereby may the temperature be affected by the component deterioration.

In papers Diakunchak (1992); Kurz et al. (2009); Kurz and Brun (2001); Brekke et al. (2009), several mechanisms that cause degradation in gas turbines are presented. The major contribution of degradation in industrial gas turbines is fouling which is caused by small particles and contaminants in the air. These particles increase the roughness of the rotor and stator surface. Another degradation effect is tip clearances which is a common diagnosis for older gas turbines. Tip clearances denotes an increasing gap between the rotating blades and the stationary casing. Fouling due to increased roughness can partially be restored by washing the compressor, while a component replacement is often needed for tip clearances. In the last paper, performed by Brekke et al. (2009), deterioration effects due to compressor fouling are investigated in an offshore industrial gas turbine application.

A common solution, about how to estimate deviation in performance from a nominal baseline is to introduce health parameters (Luppold et al., 1989; Kobayashi and Simon, 2003; Borguet and Léonard, 2008). In the two first papers, Kalman filters are used to estimate the introduced health parameters. The degradation in performance is natural, so if the model does not compensate for this degradation it can be difficult to avoid sensor false alarms. In Kobayashi et al. (2005), a nonlinear Kalman filter is demonstrated that can be used in a wide operating range for an in-flight aircraft engine diagnosis application, providing an engine model with high accuracy.

One factor that can affect the model accuracy, and especially the performance of the health parameters, is the absolute humidity in the atmospheric air. The humidity effects for an in-flight application are studied in, e.g., Bird and Grabe (1991) where methods based on parameter correction are considered. In Mathioudakis and Tsalavoutas (2002), 
a study is performed where humidity effects are investigated in an industrial gas turbine application. An analysis is presented of how the variation in ambient conditions affects the health parameter estimations. It is shown that a compensation for the ambient conditions reduces the undesirable daily variations in the estimated health parameters. In other gas turbine papers, the variation in absolute humidity is often neglected since it increase the complexity of the model and does not vary significantly much at many industrial gas turbine sites.

A fundamental effect of the change in absolute humidity is the change of molecules in the ambient air media. A change in the concentration of molecules in the media affects the thermodynamic gas properties such as enthalpy, heat capacity, and entropy. The thermodynamic properties influence the estimated performance. In the GTLib framework, as described in Section 4.6.5, the concentration of the molecules in the gas path media is changed immediately according to the change in ambient conditions. The change in ambient conditions can be encapsulated by the developed estimators to reduce undesirable daily variations in the estimated health parameters mentioned in Mathioudakis and Tsalavoutas (2002). Therefore, an evaluation is performed in Section 7.5.1 where the effect of the absolute air humidity is investigated using simulated data.

\subsubsection{Experiment Setup}

For the case studies, measurement data are collected from a gas turbine site in the Middle East. Because of the difficult environmental conditions at the site, the compressor is washed frequently. At the site, the air consists of a high grade of air pollutions such as: sand, salt, oil, and other contaminations which affect the engine's performance. The gas turbine SGT-70o at the current site is a 1-spool and a 2-shafted engine where the application is the so-called mechanical drive. A notable aspect with this specific gas turbine model is the absence of an instrumentation sensor between the gas generator and the output of the power turbine. The lack of these types of sensors makes the diagnosis and monitoring procedure more difficult, since no measurement signals are available in the gas path between the output of the compressor and the output of the power turbine. Similar gas turbines, launched by other manufactures, have thermocouples between the gas generator and the power turbine. Having ideal thermocouples in that cross-section should reduce the uncertainty of the gas path parameters in the gas generator. In the SGT-700, the thermocouples are instead installed in the exhaust gas at the output of the the power turbine.

\section{Gas Turbine Site}

A schematic view of the gas turbine site is shown in Figure 7.1 where the gas turbine and its surrounding components are presented. The dashed arrows in the figure represent physical connections, i.e., mechanical, thermodynamic, and electrical connections. Solid arrows represent signals to and from the controller.

The input signals to the controller are the measurement of the atmospheric air, the gas path instrumentation sensors, the power generated by the application, and the 


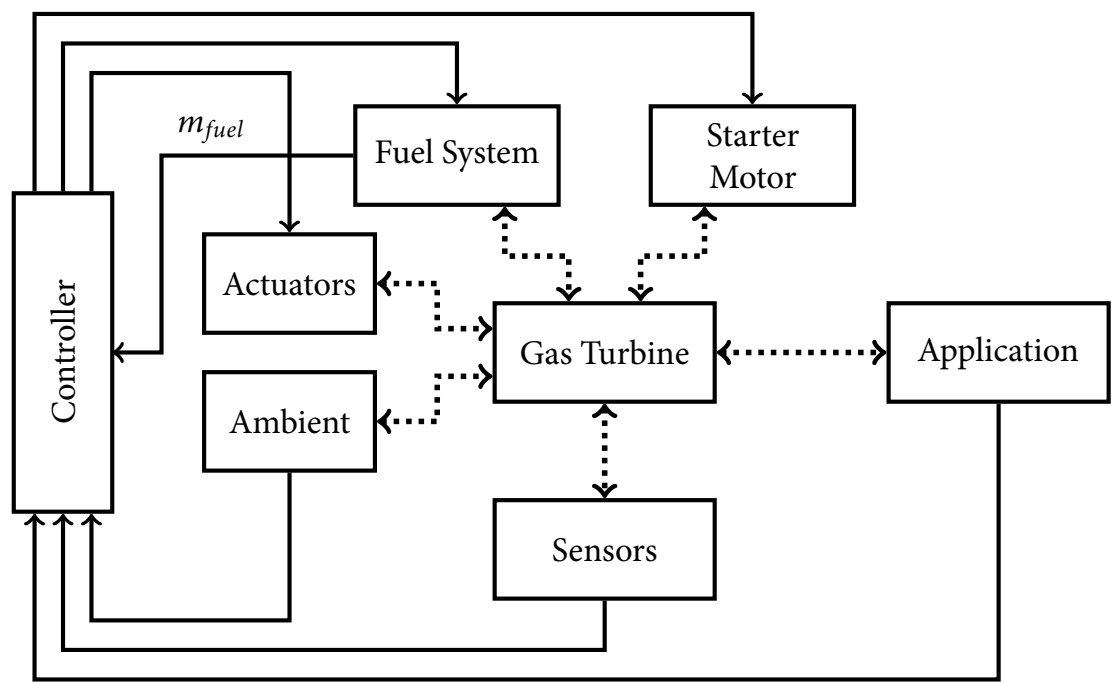

Figure 7.1: In the figure, a schematic view of the gas turbine experimental platform is shown. The dashed double arrows represent a physical connection, while solid arrows in the figure represent ordinary signals. In, e.g., Modelica, physical based connections are represented by equations and ordinary signals have only one flow direction.

desired rotational speed of the power turbine. The atmospheric air sensors measure the pressure $p_{0}$, the temperature $T_{0}$, and the relative humidity $\varphi_{0}$ of the ambient air. The instrumentation sensors measure pressures, temperatures, and shaft speeds along the engine's gas path. In Section 6.2, the exact positions of these sensors are described. Finally, the generated power by the application is not measured. Instead, the signal is estimated using internal sensors in the driven application component. Hence, the power estimation procedure does not utilize any of the gas path measurements, so a sensor fault in some of the gas path measurements does not affect the reliability of the power estimation. Thus, the power signal is here considered as a reliable non faulty measurement signal.

As shown in Figure 7.1, a measurement signal of the mass flow of fuel is available. This measurement signal does not belong to the standard site equipment since a mass flow meter is expensive for the customer to install. Thus, the mass flow meter is only installed on request by the customer. The controller use this signal (multiplied with the heat value of the fuel) to, e.g., calculate the flame temperature. When the mass flow meter is absent, the mass flow can be calculated using a model of the fuel system together with the measurement of fuel pressure and fuel valve positions. At sites where a mass flow meter is available, more information about the operation is available. This information can be included into the diagnosis system where, e.g., the estimated mass flow signal can be compared with the measured signal by the mass flow meter. 


\section{One Year of Experimental Data}

The considered sequence of available experimental data came from one year of operation. During the operation, the gas turbine is started and stopped a number of times. These startup and shutdown sequences are removed from the measurement sequence when the test quantities are evaluated since the diagnosis model is not valid during startup and shutdown. The original data have a sample time of $5 \mathrm{~min}$. To reduce the number of data points (and decrease the evaluation time), the data are re-sampled with a sample time of $60 \mathrm{~min}$. During the operation, the compressor is washed five times (middle of November, end of December, end of March, end of June, and middle of September). The time points of the compressor washes are shown in Figure 7.4 and Figure 7.9. In Figure 7.4, the washes are marked with arrows.

\section{Preliminary Investigation of the Engine's Nominal Health Status}

A preliminary study is performed where the focus is to determine the health status of the clean engine, i.e., calculate the health parameters directly after a compressor wash. With the methodology described in Section 6.6 a constant gain extended Kalman filter (CGEKF) is designed. The filter is tuned in that way so the response in the health parameters is fast. Then an approximate value of the health parameters after an compressor wash is determined. The value of the health parameters are shown in Table. 7.1.

Table 7.1: Health parameter values for a clean machine.

\begin{tabular}{cc}
\hline Health Parameter & Clean Machine [\%] \\
\hline$\Delta \eta_{C 1}$ & -3.0 \\
$\Delta \Gamma_{C 1}$ & -1.0 \\
$\Delta \eta_{T 1}$ & +3.0 \\
$\Delta \Gamma_{T 1}$ & +10.0 \\
\hline
\end{tabular}

At the present site, the engine is aged and due to the difficult environmental conditions the leakage and tip clearance in the compressor-turbine T1 is probably larger than for the reference machine which affect the performance negative. An increased tip clearance results in a lower turbine efficiency and a higher mass flow (Kofskey and Nusbaum, 1968). As Table 7.1 indicates, the value that differs most from the nominal value is for the flow capacity $C_{T}$ of the compressor-turbine $\mathrm{T} 1$ (which is proportional to the mass flow). To have all health parameters in the same range, the reference value $C_{T, \text { ref }}$ in $(5.2 \mathrm{~d})$ for the compressor-turbine $\mathrm{T} 1$ is increases with $6 \%$, which decrease the health parameter $\Delta \Gamma_{T 1}$ with 6 percent points. This value is also suggested by SIT for the present engine.

Since the exact positions of the sensors along the engine's gas path are not known and the actual absolute pressure can differ from the nominal model. Thus, a basic idea is to add a constant bias to the measurement signals to compensate for individual properties in the gas turbine fleet and to get health parameters in the same interval for a newly manufactured and cleaned machine.

The bias terms are calculated once for the cleaned gas turbine. In the experimental data sequence that is investigated, the bias terms are calculated directly after the first 
compressor wash is performed. The bias term $b_{i}$ of measurement sensor $i$ is calculated:

$$
b_{i}=\sum_{j=1}^{k} \frac{y_{i, j}^{n o m}-y_{i, j}^{m e a s}}{k}
$$

where $k$ is the number of samples that corresponds to two days of operation, $y^{\text {meas }}$ is the measured quantity, and $y^{n o m}$ is the reference value calculated from the nominal model similar to the description in Figure 7.3. The values of the bias terms, in percent of the nominal value, are shown in Table. 7.2. A positive value in the table means that the measured signal is lower than nominal value.

Table 7.2: Bias in measurement sensors.

\begin{tabular}{l|cccccccc}
\hline$y_{i}$ & $y_{p_{1}}$ & $y_{p_{3}}$ & $y_{p_{8}}$ & $y_{T_{2}}$ & $y_{T_{3}}$ & $y_{T_{7}}$ & $y_{n_{C 1}}$ & $y_{n_{T 0}}$ \\
\hline$b_{i}[\%]$ & 1 & 5 & 1 & 0 & 0 & -1 & 0 & 0 \\
\hline
\end{tabular}

The biases are added to the measurements according to:

$$
y_{i}=y_{i, \text { meas }}+b_{i}
$$

where $b_{i}$ is the constant sensor bias calculated once. In the present case, the bias compensation is more or less negligible for all sensors except for the discharge pressure sensor $p_{3}$ after the compressor. For the sensor measuring $p_{3}$, the bias compensation is about $5 \%$ from the nominal reference value (after the parameter $C_{T, \text { ref }}$ is increased). The large difference between the measurement and the nominal value for the discharge pressure does not depend on the actual sensor calibration since the discharge pressure is measured with three sensors where all the sensors show values in the same interval. An idea is that the difference in pressure came from leakages in the compressor turbine due to tip clearances.

\subsection{Introductory Methods To Detect Compressor Fouling}

The objective with this section is to investigate three simple methods that can be used to detect compressor fouling. The three first methods (a)-(c) are based on pure measurement signals, which means it is simply to make an investigation of fouling with these methods. The two first methods (a)-(b) are based on estimation of mass flow through the compressor using different sensors. The fourth method (d) is based on the performance model, and is more complex to evaluate and is presented here only for comparison. Estimation using the performance model will be presented in Section 7.3. All four methods are summarized in Table 7.3.

In all four methods, the objective is to find a so-called baseline. The baseline is determined for a number of time samples of a cleaned compressor. It is necessary to have the samples in a number of various operational points to get a proper baseline. Time samples that are collected after the nominal condition of the compressor are assumed 
Table 7.3: In the table, four methods to detect compressor fouling are presented. It is shown if the method relies on the measurements and/or the physical model.

\begin{tabular}{ll|ccc}
\hline & & $\begin{array}{c}\text { Measure- } \\
\text { ments }\end{array}$ & $\begin{array}{c}\text { Physical } \\
\text { Model }\end{array}$ & $\begin{array}{c}\text { Mass flow } \\
\text { Estimation }\end{array}$ \\
\hline (a) & Bell-Mouth Based Estimation & $\mathrm{x}$ & & $\mathrm{x}$ \\
\hline (b) & $\begin{array}{l}\text { Pressure Ratio Based Mass } \\
\text { Flow Estimation }\end{array}$ & $\mathrm{x}$ & $\mathrm{x}$ & \\
\hline (c) & Power vs. Mass Flow of Fuel & $\mathrm{x}$ & & $\mathrm{x}$ \\
\hline (d) & $\begin{array}{l}\text { Model Based Mass } \\
\text { Flow Estimation }\end{array}$ & $\mathrm{x}$ & $\mathrm{x}$ & \\
\hline
\end{tabular}

to appear below the baseline. When the distance between the samples and the baseline is sufficiently large, it is time to wash the compressor. The baselines in the Figure 7.2 are only sketched by hand and the purpose with the introduced baseline is not to get the perfect position of the baseline through minimization of some criteria. Instead, the baseline should symbolize where a possible position could be in a simplified manner.

The measurement sequence that is used in the investigation of compressor fouling detection is collected between two compressor washes. The length of the sequence is about three months, where the first 40 samples start in January, and the last 40 samples end in March. These samples are marked in Figure 7.2 together with all the considered points. The first samples (clean compressor) should be used to span the baseline, and the last samples (fouled compressor) should be used to detect the fouled compressor. In all Figures 7.2(a)-(d), normalized quantities from (5.2) are used.

\subsubsection{Bell-Mouth Based Estimation}

In Diakunchak (1992), three methods to detect deterioration due to compressor fouling are presented. One of these methods descends from an estimation of the mass flow through the compressor, and a common approach to calculate the mass flow is to measure the static pressure drop over the inlet duct of the compressor, i.e., over the bell-mouth. To get a reliable estimation of the absolute mass flow amplitude, the bell-mouth measurements need to be calibrated, together with the inlet pressure and the inlet temperature. For monitoring purposes, it is enough to consider the relative changes in mass flow, and according to Scott (1986), bell-mouth based measurement is a good technique to detect compressor fouling. It is assumed that the mass flow is proportional to the square root of the pressure drop over the bell-mouth. Thereby a decreased pressure drop over the bell-mouth, for a given normalized rotational speed can be interpreted as an increasing deterioration due to compressor fouling. To find when it is time to wash the compressor, a baseline of a cleaned compressor need to be constructed. This can be done by running the gas generator for a number of different rotational speeds, and then plot the actual baseline for these speeds. Points that appear below this baseline indicate a fouled 
compressor, and when these points have dipped to a certain value, it is time to wash the compressor.

In Figure 7.2(a), the pressure loss measurement over the bell-mouth is plotted versus the normalized speed of the gas generator. In the figure, it is shown that it can be difficult to find a proper baseline, since the cloud of points are lumped, scattered, and cover nearly the same area. Thus for this specific sequence the bell-mouth measurements are no good to determine compressor fouling.

\subsubsection{Pressure Ratio Based Mass Flow Estimation}

For the case with a constant compressor pressure ratio together with fixed ambient conditions, the compressor need to rotate faster to compensate for the degradation in efficiency due to fouling (Kurz et al., 2009). At the same time, the compressor will also consume more power and fuel. Therefore it is interesting to study the pressure ratio versus the normalized rotational speed of the compressor. In Figure 7.2(b), the same measurement sequence is plotted as for the bell-mouth measurement, but the compressor ratio is instead considered. As Figure 7.2(b) shows, it is easier to find a baseline in the pressure ratio plot than in the previous plot based on bell-mouth measurements. All the points that should represent a fouled compressor appear in the lower interval in Figure 7.2(b), which is desirable. It is undesirable that points that appear in the upper interval are not representative for a clean compressor. Finally, a considered baseline is sketched in the figure together with all available points.

\subsubsection{Power versus Mass Flow of Fuel}

In Figure 7.2(c), the power generated by the application is plotted versus the mass flow of fuel. This test case can be intuitive when it is assumed that the overall efficiency of the engine and the heat value of the fuel are nearly constant for all operation points. For this test case, the points that symbolize a fouled compressor appear in the lowermost layer seen from the baseline.

\subsubsection{Performance Model Based Mass Flow Estimation}

According to Meher-Homji (1987), a mass flow meter is preferred against a mass flow estimation based on bell-mouth measurement, as an indicator of fouling. For the case where the mass flow through the compressor is not measurable, model based techniques can be utilized to calculate the actual mass flow. These techniques utilize the thermodynamic heat and mass balances. Here, the performance model constructed in GTLib package is simulated with the actual input signals shown in, e.g., Figure 6.3, to determine the mass flow of air. The result of the study is presented in Figure 7.2(d), and shows good result where all samples before the compressor wash appear in the lowermost layer seen from the baseline. The data points which represent fouled and cleaned conditions are also separated. 


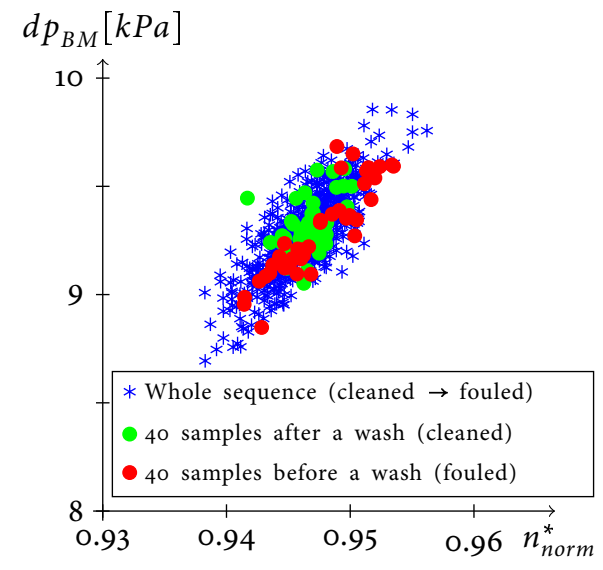

(a) Bell-mouth pressure loss versus normalized speed of the compressor.

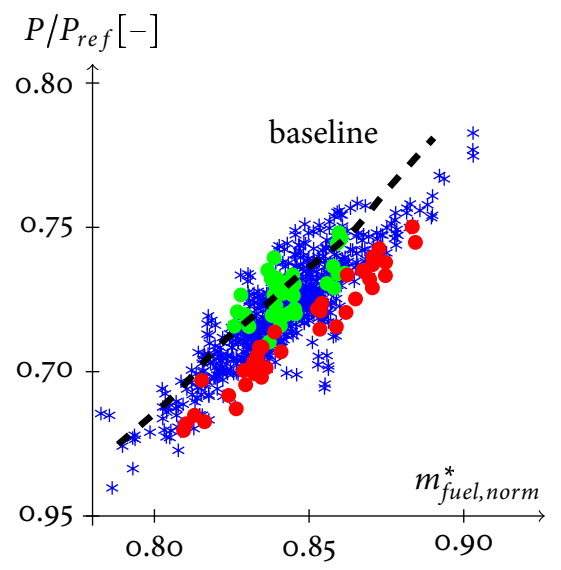

(c) Generated power by the application versus the normalized mass flow of fuel.

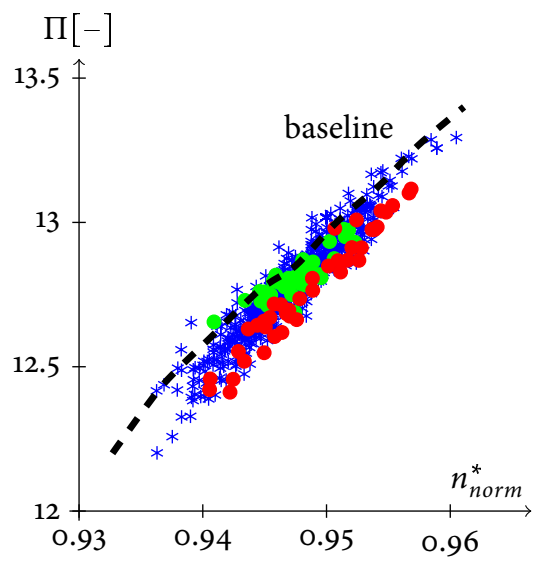

(b) Pressure ratio versus normalized speed of the compressor.

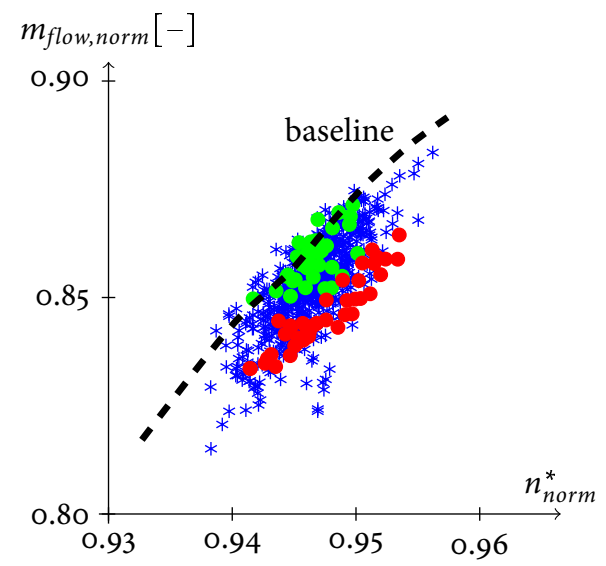

(d) Normalized mass flow of air through the compressor versus the normalized speed.

Figure 7.2: In the figure, four techniques to detect a fouled compressor are presented. The considered measurement sequences are collected between the two compressor washes that are performed at the beginning of January and at the end of March. All presented signals are based on measurements except the signal of the normalized air flow rate $m_{\text {flow, norm }}$ through the compressor. The mass flow of air is calculated by the gas turbine model, which is based on the mass and heat balances. The normalized quantities are defined in (5.2). The objective with the study is to find a baseline that can be determined for a clean machine (green points) for a number of different operational points. In the figures, the baseline is only sketched by hand to illustrate the principle. Before the compressor wash, the measurement points should be below the baseline (red points). According to this principle, (d) gives the best result and (a) gives the worst result. 


\subsection{Measurement Delta}

In Urban (1972); Urban and Volponi (1992); Simon et al. (2008), the so-called Measurement deltas are introduced. A brief introduction of these measurement deltas is available in the gas path analysis Section 6.1.1. The measurement deltas are an important part of the gas path analysis (GPA). The deltas are assumed to capture the deviation from a nominal baseline for a given number of known signals (or combination of known signals). For monitoring purposes, the deltas can be used to supervise trends or detect abrupt changes that can indicate a unhealthy behavior of the engine. Since the reference model, which is considered herein, consists of a number of corrected parameters and performance maps the delta calculation in Figure 6.1 can be replaced with the diagram in Figure 7.3.

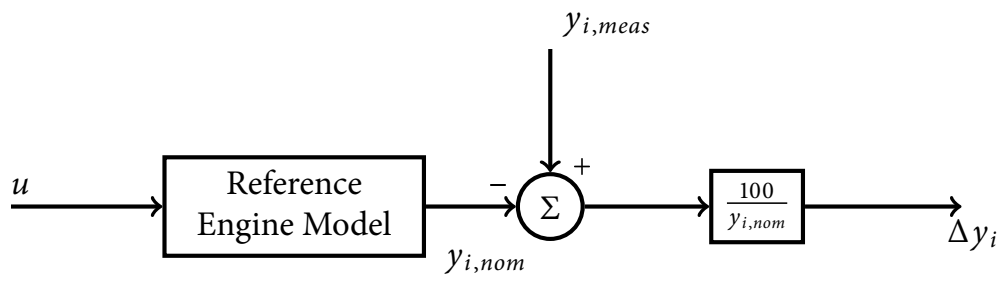

Figure 7.3: Measurement delta $\Delta y_{i}$ calculation of the measured quantity $i$.

The measurement deltas are written:

$$
\Delta y_{i}=100 \frac{y_{i, \text { meas }}-y_{i, \text { nom }}}{y_{i, \text { nom }}}
$$

where $y_{i, \text { meas }}$ represents the measured value, and $y_{i, \text { nom }}$ represents the nominal value of the measured quantity $i$. The results of the delta calculation (of one year of experimental data) for the measured discharge temperature $\Delta T_{3}$ of the compressor and the exhaust gas temperature $\Delta T_{7}$ after power turbine are shown in Figure 7.4 and Figure 7.5.

It is possible to construct deltas for other types of quantities, e.g., mass flows and efficiencies. It can be difficult to obtain these quantities since relevant parts of the performance model need to be chosen. Here, only deltas of the measured quantities are constructed. The deltas which give the best visible results are for the measured temperatures. It is possible to separate trends due to the compressor washes in the deltas which Figure 7.4 and Figure 7.5 indicate. These trends can be monitored by an FDI-system to decide when a compressor wash is necessary to perform. The best visible result is shown for the $\Delta T_{7}$ measurement. A disadvantage with the deltas is that the ambient conditions, e.g., the atmospheric air temperature shown in Figure B.1 may impact the estimations. Compare, e.g., the absolute value of the deltas for the compressor washes in the end of December and in the end of June. This complicate the usage of a static threshold to detect compressor fouling.

It is desirable to have a static threshold $L_{w}$ to detect when it is time to wash the compressor due to fouling. The compressor should be washed regularly when a certain degree of fouling has occurred. As seen in Figure 7.4 and Figure 7.5 a static threshold $L_{w}$ 


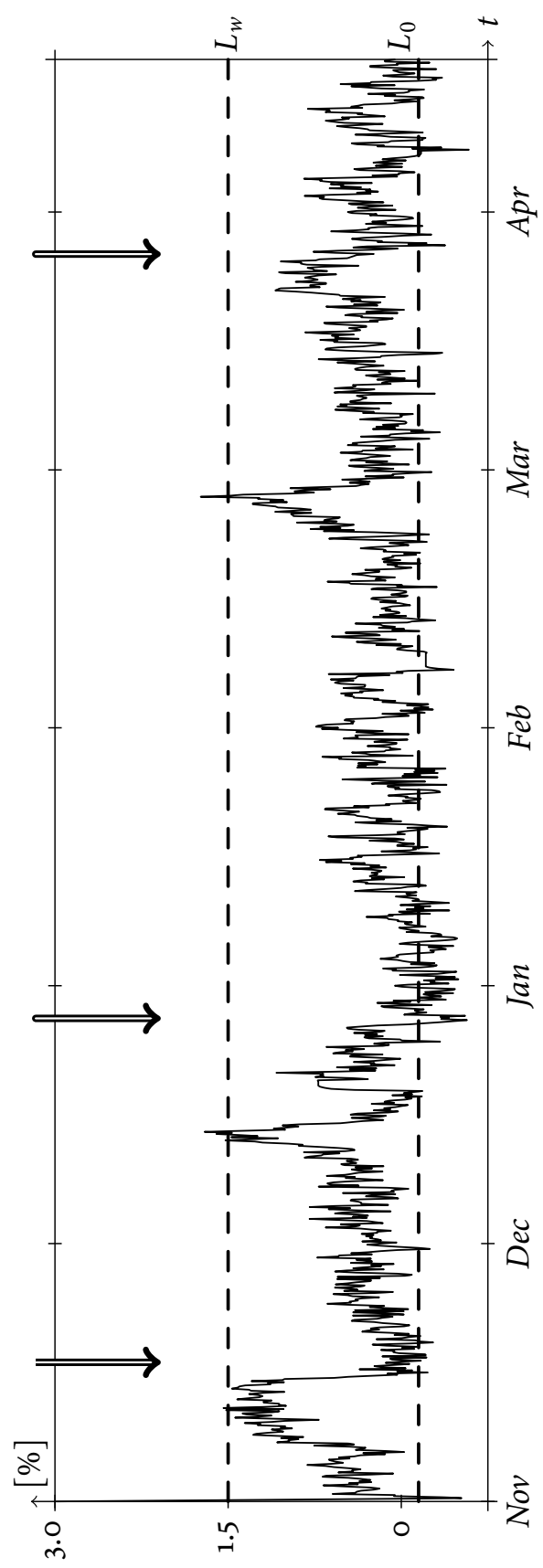

(a) $\Delta T_{3}$ for the first six-month period.

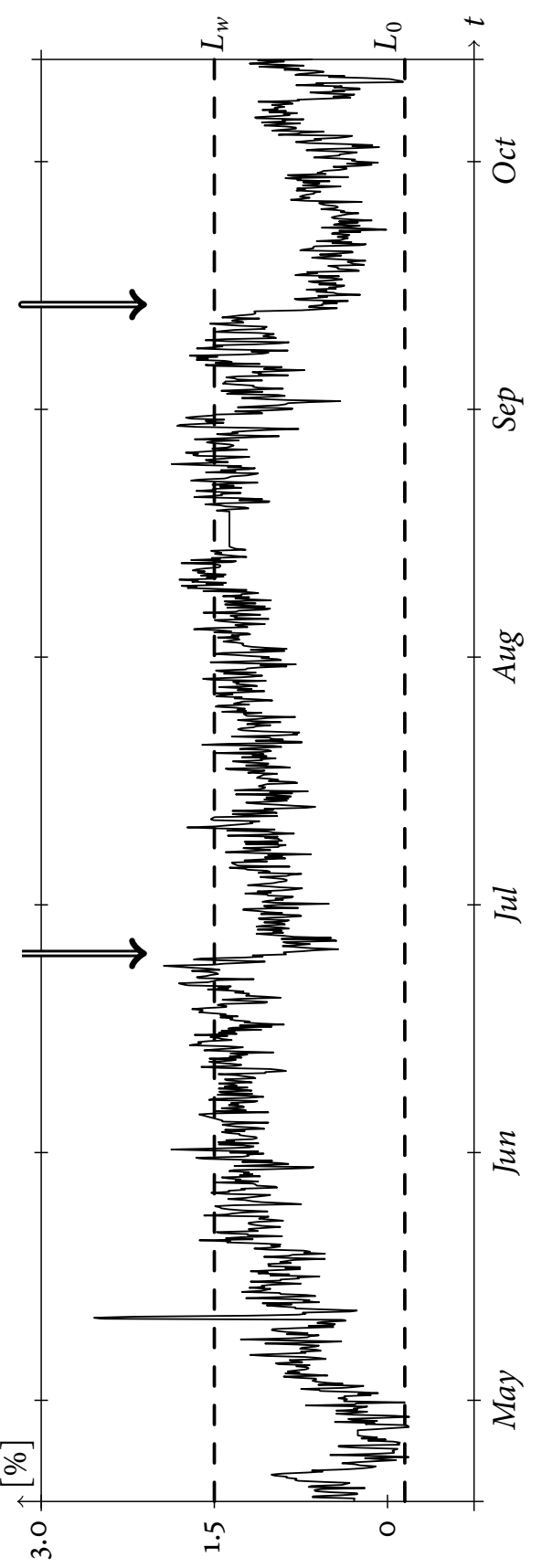

(b) $\Delta T_{3}$ for the second six-month period.

Figure 7.4: In the figure, the delta calculation $\Delta T_{3}$ together with the given static thresholds are shown. The upper static threshold is used to detect compressor fouling, while the lower static threshold should indicate the performance level of a cleaned compressor. 


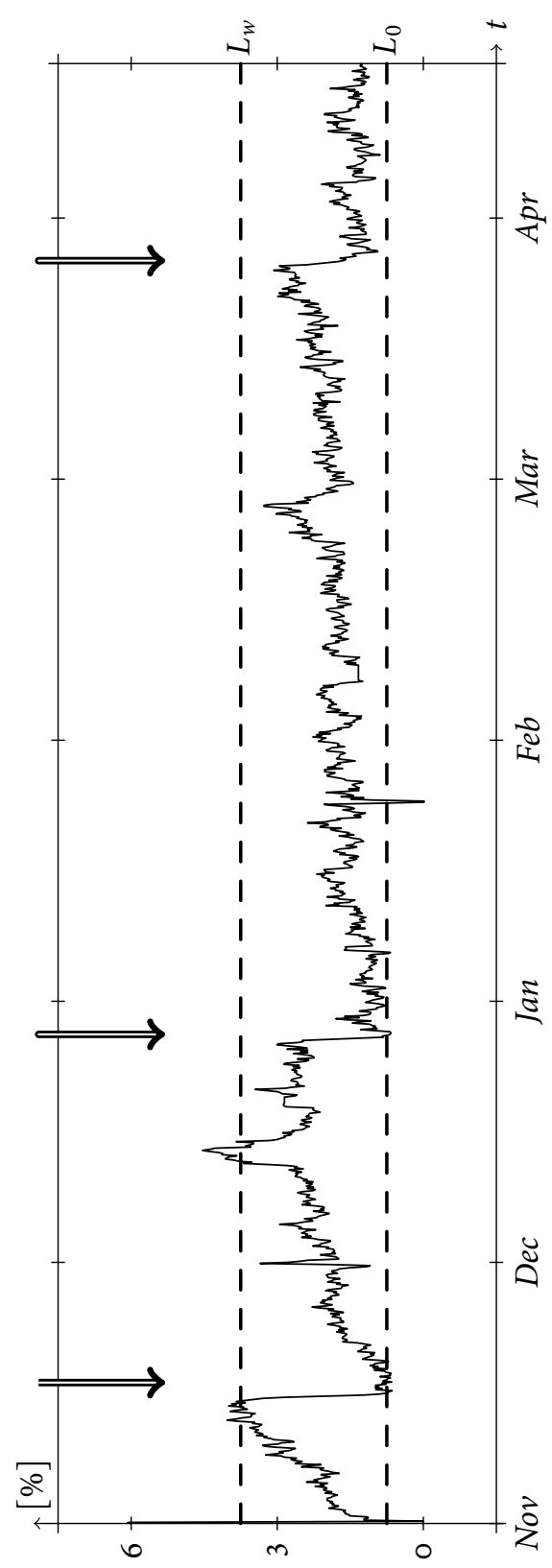

(a) $\Delta T_{7}$ for the first six-month period.

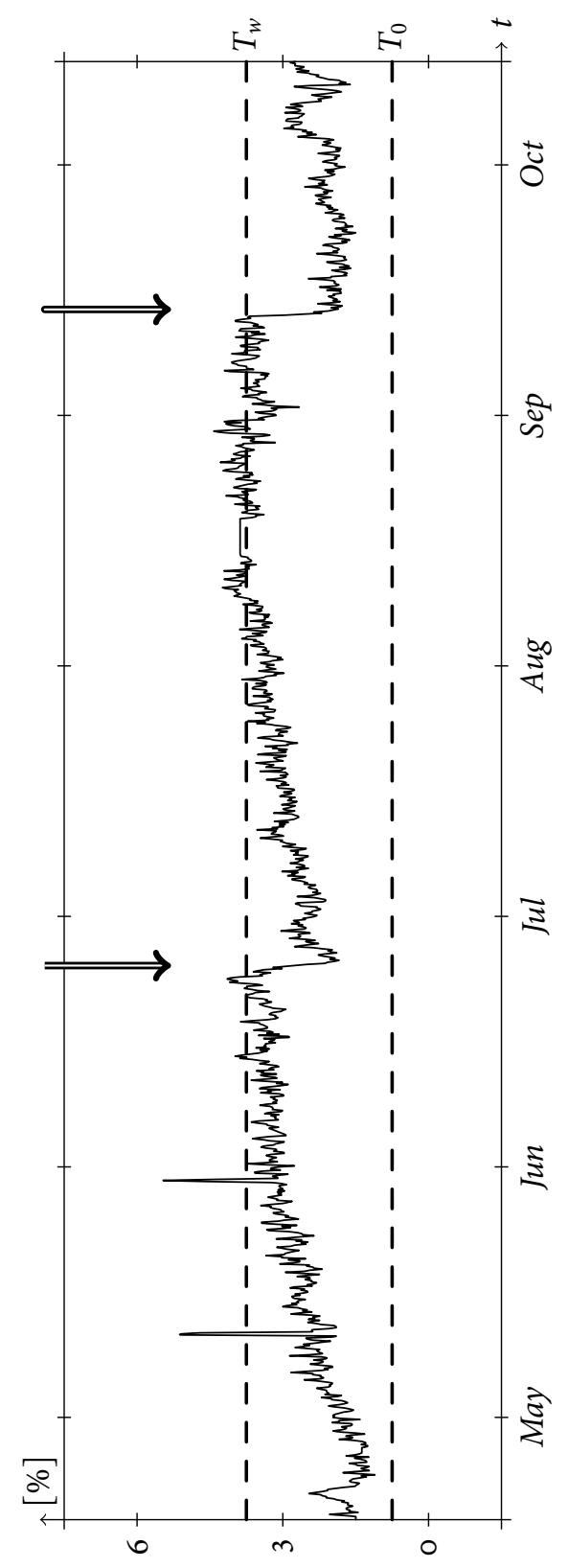

(b) $\Delta T_{7}$ for the second six-month period.

Figure 7.5: In the figure, the delta calculation $\Delta T_{7}$ together with the given static thresholds are shown. The upper static threshold is used to detect compressor fouling, while the lower static threshold should indicate the performance level of a cleaned compressor. 
used to trigger a compressor wash is not suitable since the delta levels are different for the summer and winter half year. Also the relative change of the deltas between the washes are different, e.g., the performance restored by the wash in the middle of November is larger than for the wash in the end of June.

The plot results can be misleading since the status of fouling before each wash is not exactly known. Thus, how fouled the compressor is can be different before each wash is performed. On the other hand, after a compressor wash the degree of fouling should be the same independently of the winter or the summer period since it is assumed that the same washing procedure is utilized. The lower threshold $L_{0}$ in the figures suggests that the estimations are not independent of the ambient air condition. Especially, slow changes in increasing atmospheric air temperature can be difficult to be discriminated from a cleaned compressor, and vice versa. This can clearly be seen at the end of February in the figures where the deltas have increased abnormally much, which can be misunderstood as a fouled compressor.

\subsection{Constant Gain Extended Kalman Filter}

The Kalman gain is calculated in the operating point where: (i) the ambient air conditions are at datum state, (ii) the power $P_{A}$ generated by the generator is $21 \mathrm{MW}$, (iii) the speed of the power turbine is given by the generator at $50 \mathrm{~Hz}(\approx 6500 \mathrm{rpm})$, and (iv) the mass flow of fuel (with a lower heating value of about $50 \mathrm{MJ} / \mathrm{kg}$ ) is calculated using the simulation platform for the above input signals. In the present work, a mechanical drive application is studied which results in a power turbine which can have a variable speed. A sketch of the CGEKF together with the gas turbine and its environment components is shown in Figure 7.6. The output signals from the observer component are: (i) the estimated health parameters $\hat{h}$, (ii) the estimated combustion temperature $\hat{T}_{f}$, (iii) the estimated measurement signals $\hat{y}$, and (iv) an estimation of all the other states variables $\hat{x}$.

\subsubsection{Observability}

In the gas turbine diagnosis model, eight unique measurement positions along the engine's gas path are available. According to Lemma 6.5.4, the maximum number of health parameters that can be considered in the model is eight. If more health parameters are used, the observability of the system is not reached. Since the requirement in Lemma 6.5.4 is necessary but not sufficient, the number of health parameters that are observable could be smaller than the maximum number specified in the Lemma. Thus, an observability analysis of the gas turbine diagnosis model is necessary to perform.

To check the observability of the diagnosis model, with different health parameter configurations, the linearized model is studied. According to the numerical problems that can appear for larger models when observability is checked with the criteria in Theorem 6.5.1, the structural observability criteria in Theorem 6.5.2 (Shields and Pearson, $1976)$ is used. The structural observability analysis shows that the health parameter configuration, described in (6.7), is structurally observable in the linearization point of the chosen CGEKF observer. If another health parameter, i.e., for the efficiency and mass 


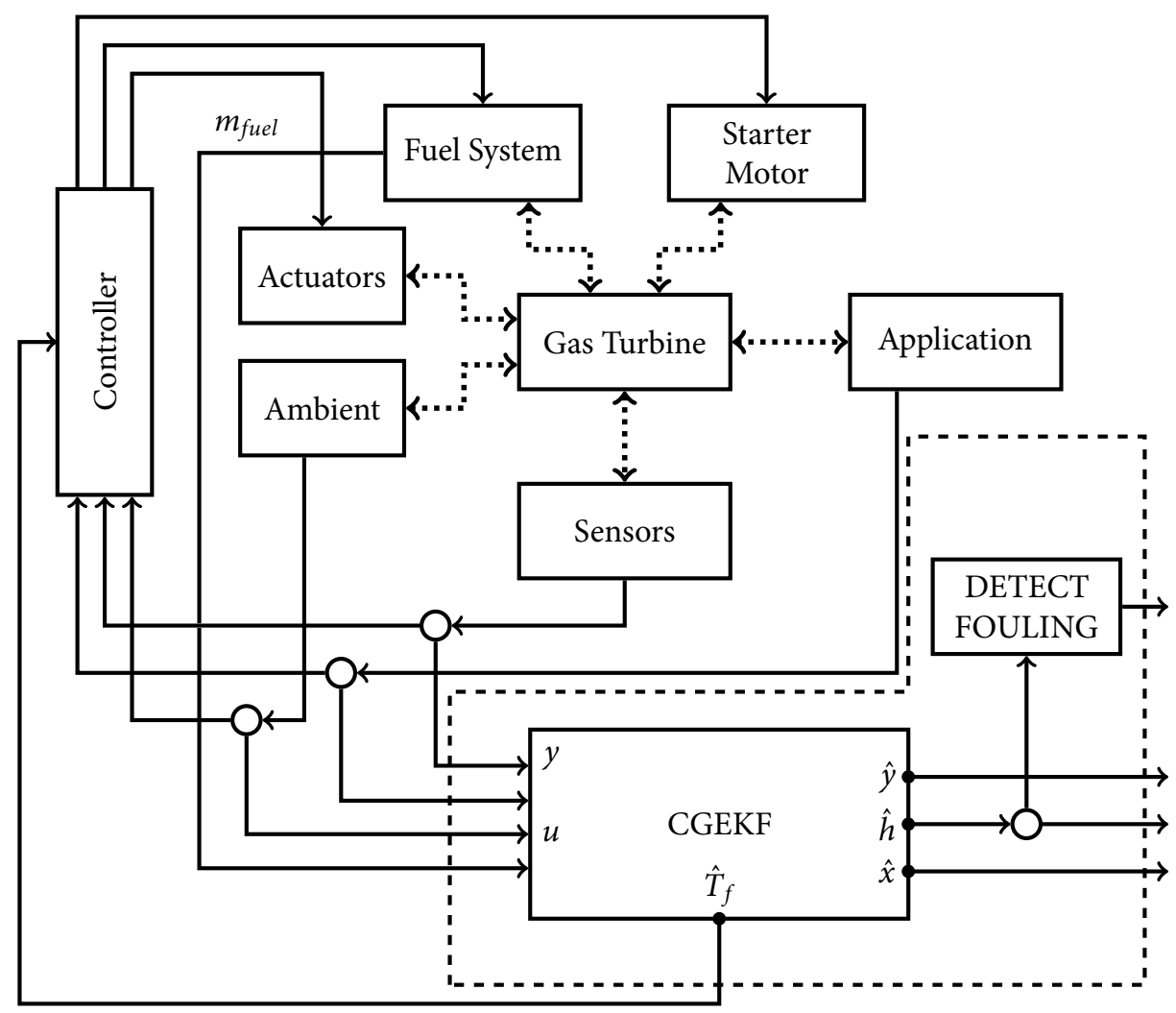

Figure 7.6: Schematic view of the gas turbine experiment platform, where the Constant Gain Kalman filter is introduced to estimate the gas turbine health $h$ and the combustion temperature $T_{f}$. The health estimation is used to detect fouling and the combustion temperature is used by the controller to supervise the turbine inlet temperature (TIT). The estimation of $\hat{y}$ can be used to, e.g., detect an abrupt sensor or actuator fault. 
flow through the power turbine is added, or the two fault in the input signals $P_{A}$ and $n_{A}$, the linearized model is no longer structurally observable which induce unobservability. For the case where the two health parameters for the inlet and outlet duct are added, the structural observability is not affected. A health parameter in the inlet duct can for example be interpreted as a pressure drop in the air filter due to, e.g., fouling. This indicates that it can be possible to supervise the health parameter. Finally, the structural observability of the linearized diagnosis model is strongly connected to the number of health parameters, and where in the model these parameters are introduced.

\subsubsection{Observer Tuning}

The covariance matrices $Q$ and $R$ affect the estimation of the state vector $\hat{x}$ in the filter. When the uncertainties of the measured signals are independent, the $R$ matrix is diagonal. The diagonal elements of the matrix represent the variance of respective measurement signal. The standard deviation are calculated for a number of samples directly after a compressor wash for a given stationary point. It appears that the standard deviation lies in the interval 1-3\% of the sensor reference value in the operating point. This gives matrix elements in $R$ as:

$$
R_{i, i}=\left(r_{i} y_{r e f, i}\right)^{2}
$$

where $i=1, \ldots, 8$, and $r_{i}$ represents the uncertainty of sensor $i$. The model uncertainty matrix $Q$ is determined in a similar manner according to:

$$
Q_{j, j}=C_{q}\left(q_{i} x_{r e f, i}\right)^{2}
$$

where $j=1, \ldots, n, q_{i}$ represents the uncertainty, and $n$ is the number of state variables. Since the reference values of the health parameters are zero, the $x_{r e f, i}$ for these parameters have the value according to the characteristics in the nominal case. All $q_{i}$ :s are given the same uncertainty of $1 \%$ except the $q_{i}$ :s of the health parameters which are given much smaller values since the component deterioration is slow. When the constants $r_{i}$ and $q_{i}$ are specified, the relation between $Q$ and $R$ can be adjusted using the scalar parameter $C_{q}$. With this scaling factor it is easy to make a compromise between the noise sensitivity and the response of the estimator. The most important parameters are the $q_{i}$ :s which represent the model uncertainty of the health parameters. When the health parameters are varying too slowly, the component deterioration appears in the estimation errors $y-\hat{y}$ which is not desirably and implies that the $q_{i}:$ s of the health parameters are tuned incorrectly. Thus, the $q_{i}$ :s of the health parameters are increased.

The method to tune the filter is not an optimal procedure but is a simple enough to construct Kalman filters without using the exact values of the uncertainty matrices, since they are unknown. A stop criterion when the tuning is complete is when the residuals $\left(r_{i}=y_{i}-\hat{y}_{i}\right)$, roughly speaking, have no visible trends and the values are centered around the $x$-axis. 


\subsubsection{Filter Design Summary}

The filter design procedure consists of four important steps. These steps are: (i) the index reduction, (ii) the over-determined $\mathrm{M}^{+}$part, (iii) the state space form, and (iv) the filter construction. A summary of the design procedure is:

1. Index reduction:

(a) Start with the model given in (6.13). Since the measurement signal $y$ is a known signal vector, the system is over-determined. Thus, remove the measurement equation $y=\bar{h}(x)$ to get a system that is exactly determined.

(b) Acquire the structural model of the system.

(c) Check, and reduce the DAE-index of the system (if necessary). For this step, Pantelides algorithm (Pantelides, 1988) is invoked. The input to the algorithm is the structural model. The output of the algorithm is the equations that need to be differentiated to receive a smaller index problem.

(d) Take back the removed measurement equations from the first step.

2. Find the over-determined $\mathrm{M}^{+}$part:

(a) Once again, acquire the structural model of the system. This time, the measurement equations are also included.

(b) Find an over-determined part of the structural model. For this step, the Dulmage-Mendelsohn decomposition (Dulmage and Mendelsohn, 1958) is performed where the whole over-determined $\mathrm{M}^{+}$part is chosen.

(c) The actual test equations are now selected.

3. The state space form:

(a) Write the semi-explicit DAE-index 1 system in an ordinary state space form through an inversion of the algebraic constraints and a symbolic transformation of the $E$ matrix in (6.21a). Some of the algebraic constraints are nonlinear so they are solved with a nonlinear numerical solver.

(b) Linearize the system in a suitable operating point and calculate the matrices $A$ and $C$.

(c) Check the structural observability of the linearized system.

4. The filter construction:

(a) Specify the measurement uncertainty matrix $R$, and the model uncertainty matrix $Q$, for the Kalman filter where the elements represent the health parameters are given small values.

(b) Calculate the Kalman gain $K$, through solving the Riccati equation (6.35), for the given uncertainty matrices $Q$ and $R$.

(c) Implement the developed Kalman filter. 


\subsection{Case Studies}

In this section, the design of an observer based CGEKF estimator is evaluated using (i) data generated from the simulation platform (Evaluation 1), and (ii) experimental data collected from a gas turbine mechanical drive site (Evaluation 2-3). The objectives of the simulation study are to: (i) see if the injected performance deteriorations can be estimated by the designed CGEKF estimator, and (ii) investigate how different ambient conditions affect the estimations. The objectives of the experimental case studies are to: (i) estimate the actual health state of the gas turbine over time, (ii) see if the CGEKF gives reliable state estimates, and (iii) check if the CGEKF based concept is suitable to use when the time for a compressor wash is determined. In the evaluation, four health parameters are introduced in the diagnosis model according to Figure 6.3. These health parameters represent: (i) efficiencies, and (ii) flow capacities of the compressor $\mathrm{Cl}$ and compressor-turbine $\mathrm{T} 1$.

\subsubsection{Evaluation 1: Atmospheric Weather Condition Dependence}

The objectives in this evaluation are to: (i) assure that the CGEKF converges to reasonable state values during a sweep in different operating points simultaneously deteriorations in performance are introduced, and (ii) investigate the difference between an observer that compensates for changes in absolute humidity in the incoming air and one observer that does not compensate for changes in absolute humidity in the incoming air. Incoming gases affect the performance of the gas turbine model; therefore it is interesting to investigate how large the effects in performance are when the air humidity is changed. In Mathioudakis and Tsalavoutas (2002), a study is presented where the variation in humidity has a negative effect on the health parameter estimation. The observer that does not compensate for change in absolute humidity is developed for the datum atmospheric conditions such as: $p_{0}=1.013 \mathrm{bar}, T_{0}=25 \mathrm{C}^{\circ}$, and $\varphi_{0}=60 \%$. These environment values give an amount of water in the incoming air according to the moist air model presented in Buck (1981). The numerical values for different ambient conditions are shown in Table 5.1.

Input data for the two filters are collected from the reference platform shown in Figure 2.5. In this evaluation study, the ambient temperature is varied according to the interval $15-35 \mathrm{C}^{\circ}$ and the relative humidity is varied according to the interval $40-80 \%$ when the pressure is at the datum state. At the same time, the power generated by the external application is varied in the interval 16-26 MW. Because of the varied power, the speed of the gas generator GG is also varied. In this evaluation study, an electric generator with fixed frequency is used. Thus the power turbine has a constant speed, but different generator frequencies are investigated in other studies that have been performed, and with similar results of the estimated health parameters. The objective is to estimate deviation in performance and therefore a deterioration in the considered performance parameters is injected. These injected deteriorations, in percent from respective baseline reference value are presented in Table 7.4.

The results from the simulation study is presented in Figure 7.7, where the estimated health parameters are shown. In the Figures $7.7(\mathrm{~b})-(\mathrm{e})$ the estimated degradations in the 
Table 7.4: Injected deterioration in performance equations.

\begin{tabular}{cc}
\hline Health Parameter & Injected Deterioration [\%] \\
\hline$\Delta \eta_{C 1}$ & -3.4 \\
$\Delta \Gamma_{C 1}$ & -2.5 \\
$\Delta \eta_{T 1}$ & +2.4 \\
$\Delta \Gamma_{T 1}$ & +4.6 \\
\hline
\end{tabular}

gas path components are shown. Figure 7.7(a) shows the generated power and the amount of water in $1 \mathrm{~kg}$ atmospheric air. The highest and lowest values denote the two extreme cases from Table 5.1. For these two extremes, the observer that does not compensate for absolute humidity has a variation in the health estimation of about 1-2 percentage points for all cases except for the efficiency of the compressor turbine.

The observer that compensates for atmospheric weather conditions follows the injected deterioration in the reference platform nearly perfect even when the ambient conditions are changed, except during transients. One explanation of this phenomenon is that the gas properties, according to the change in atmospheric air, are updated simultaneously in the whole observer. It can also be seen in the figure that different operating points do not affect the estimations so much, which is desirable. In Figure 7.7, the power turbine has a fixed speed, but similar simulation studies are performed where also the power turbine speed is varied. The results of these studies are similar with the outcome in Figure 7.7. The conclusion is that the observer that compensates for the different ambient conditions gives better performance estimations and converges to the actual state of the system also in the case when the speed of the power turbine is varied.

\subsubsection{Evaluation 2: State Estimation for Control}

An important signal for the controller during operation is the flame temperature $T_{f}$. Since the temperature is not measured, the signal has to be estimated, e.g., by an observer. An incorrect estimate of this signal can result in a too high turbine inlet temperature (TIT) which can lead to unnecessary heat stresses in the turbine blades. When the compressor deteriorates due to, e.g., fouling the compressor discharge and turbine inlet temperatures increase. It is desirable to run the machine with so high TIT as possible. Thus, if the component deteriorations are known it is easier to maintain the optimal TIT. Therefore, it is interesting to see how the fouling affects the flame temperature $T_{f}$ using the experimental data together with the model.

An investigation is performed where the flame temperature $T_{f}$ is compared for two CGEKFs. The first CGEKF does not compensate for the deterioration, and the second CGEKF does compensate for the deterioration using the health parameters. The two filters have the same $Q$ and $R$ matrices except for the rows and columns which represent the health parameters. The result of the investigation is shown in Figure 7.8, where the 


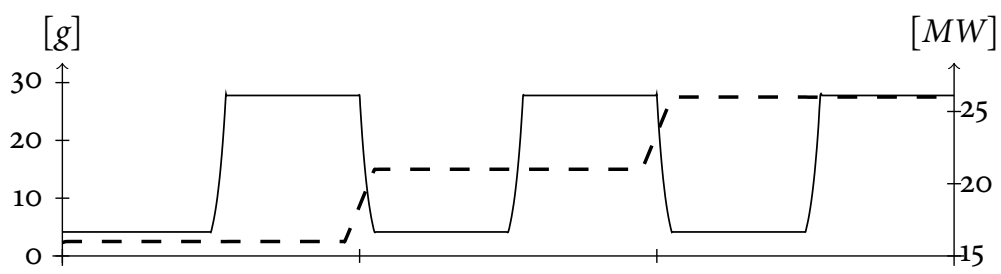

(a) Water steam - solid, Generated power - dashed

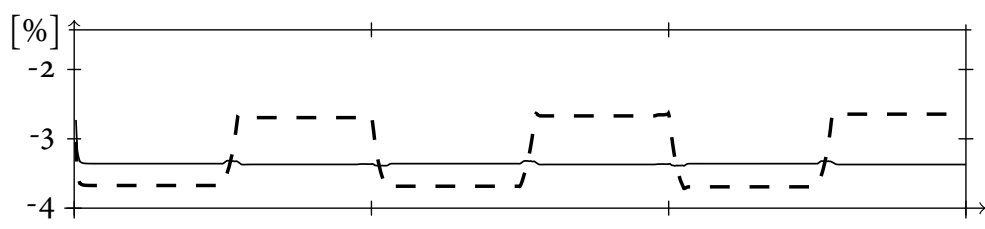

(b) Health parameter: $\Delta \eta_{C 1}$

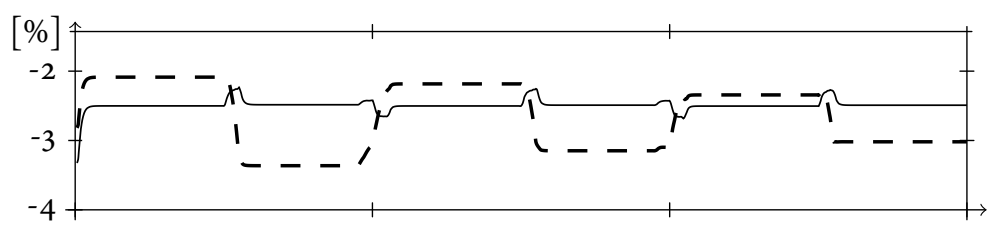

(c) Health parameter: $\Delta \Gamma_{C_{1}}$

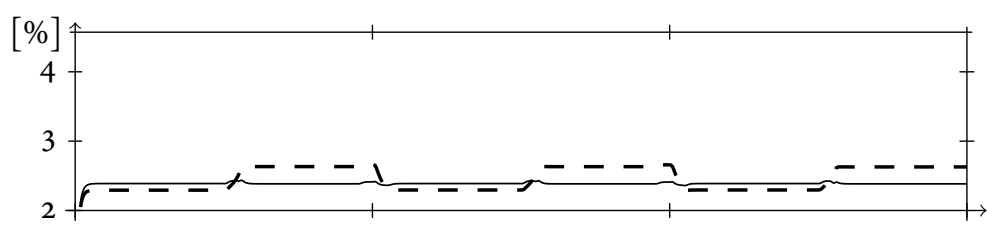

(d) Health parameter: $\Delta \eta_{T 1}$

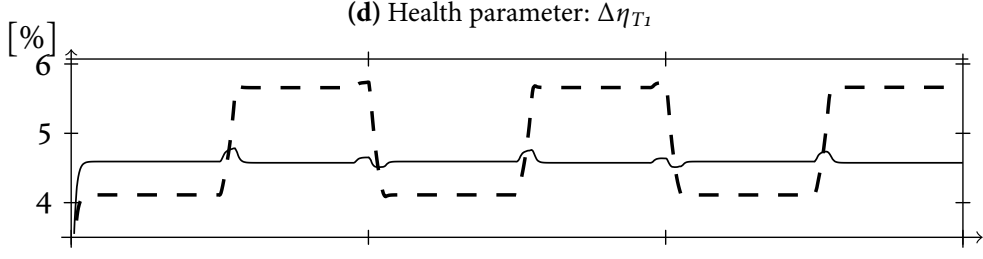

(e) Health parameter: $\Delta \Gamma_{T_{1}}$

Figure 7.7: In the figure, performance estimation of data generated in simulation platform shown in Figure 2.5, with injected degradations according to Table 7.4 (not viewed here), are shown. Two CGEKF observers are evaluated, i.e., an observer that compensates (solid lines) and an observer that does not compensate (dashed lines) for the variation in ambient conditions are shown in (b)-(e). The power of the external application is varied together with changes in the absolute humidity of the incoming air are viewed in (a). 
difference between the turbine inlet temperatures for the two CGEKFs is defined as:

$$
\Delta T_{f}=\frac{T_{f, 4}-T_{f, 0}}{T_{f, 4}}
$$

where $T_{f, 4}$ is the flame temperature for the filter that does compensate for the deterioration, and $T_{f, 0}$ is the flame temperature for the filter that does not compensate for the deterioration. In Figure 7.8 it is shown that the difference in temperature of the two filters will be up to $1.5 \%$. The figure shows that the CGEKF with four health parameters estimates a higher temperature than the CGEKF with no extra health parameters. The difference between the two estimates varies also with the component deterioration. For a clean machine, the temperature estimates are almost the same but start to deviate according to the increased deterioration. Thus, if $T_{f, 0}$ is used in the controller, then a variable temperature threshold which depends on the actual deterioration in the engine is needed to maintain reliable TIT. Instead, if the temperature $T_{f, 4}$ is used, then the deterioration is compensated using the health parameters which results in a more reliable temperature estimation. The temperature $T_{f, 4}$ assumes to be more reliable since the estimation errors (residuals) are smaller for the CGEKF that compensates for the deterioration than for the CGEKF that does not compensate for the deterioration as Figure 7.8(b)-(d) indicates. In total, there are eight residuals but only three of them are shown in the figure.

\subsubsection{Evaluation 3: State Estimation for Monitoring}

To maintain good availability and reliability of the operation it is important to supervise the engine's performance. The performance depends of the efficiencies and mass flows in the gas path components. These parameters are not measured, thus they need to be estimated by, e.g., an observer. In the present application, the deviation in efficiency and mass flow from a baseline is estimated by the CGEKF. For the compressor, the estimation results are shown in Figure 7.9. The data are collected during one year of operation and the compressor is washed five times: (i) mid November, (ii) end of December, (iii) end of March, (iv) end of June, and (v) mid September.

To determine when it is time to wash the compressor, a good idea is to study the health parameters $\Delta \eta_{C 1}$ and $\Delta \Gamma_{C 1}$. These parameters vary in the interval length of two percentage points. The interval is marked with the upper and lower thresholds $L_{0}$ and $L_{w}$ to match the estimated deviation, where the lower threshold $L_{w}$ can be used to trigger a compressor fouling alarm in the FDI-system. In Scott (1986), a compressor wash is suggested when the compressor efficiency has deteriorated $3 \%$. At SIT, a common procedure is to wash the compressor before it has deteriorated $3 \%$. Thus, a rule of thumb is to wash the compressor when the performance has deteriorated $2-3 \%$. Since the true deterioration is unknown, it is not possible to know how fouled the compressor was when the washes were performed. However, the time distances between the washes are different, i.e., the second wash is performed after 45 days and the third wash is performed after three months. Before the third wash (end of March), the performance has not deteriorated as much as in the other cases according to the estimations. A possible explanation for this can be that the distance to the previous wash is too large although 


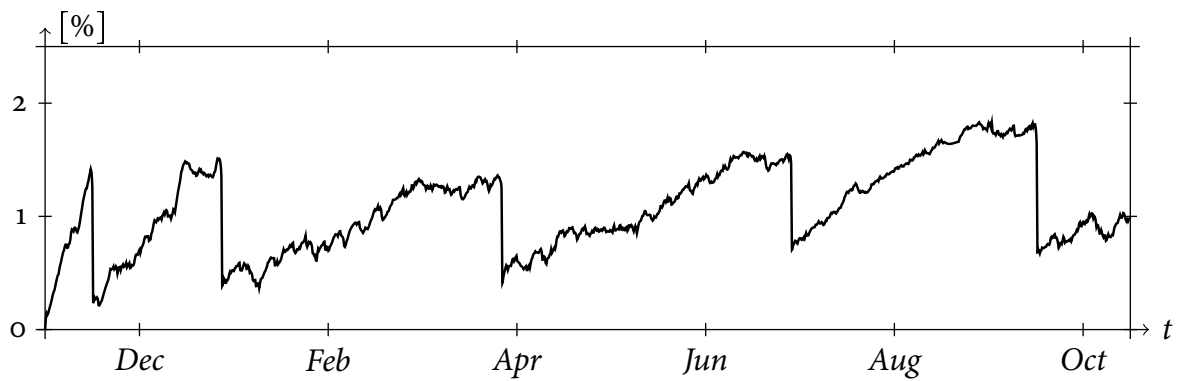

(a) Flame temperature difference $\Delta T_{f}$

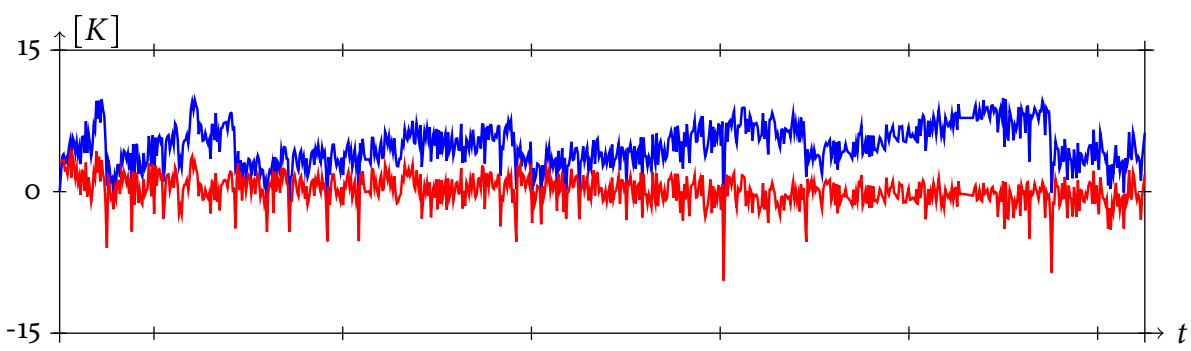

(b) Residual $r_{T_{3}}=y_{T_{3}}-\hat{y}_{T_{3}}$

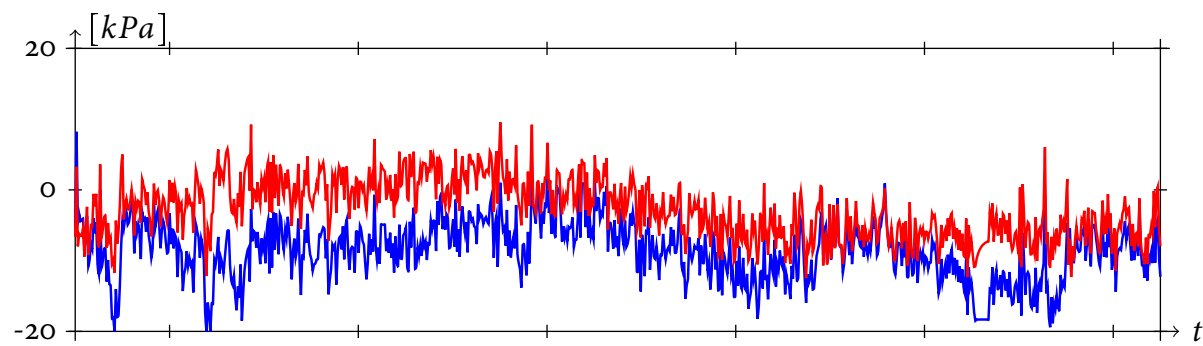

(c) Residual $r_{p_{3}}=y_{p_{3}}-\hat{y}_{p_{3}}$

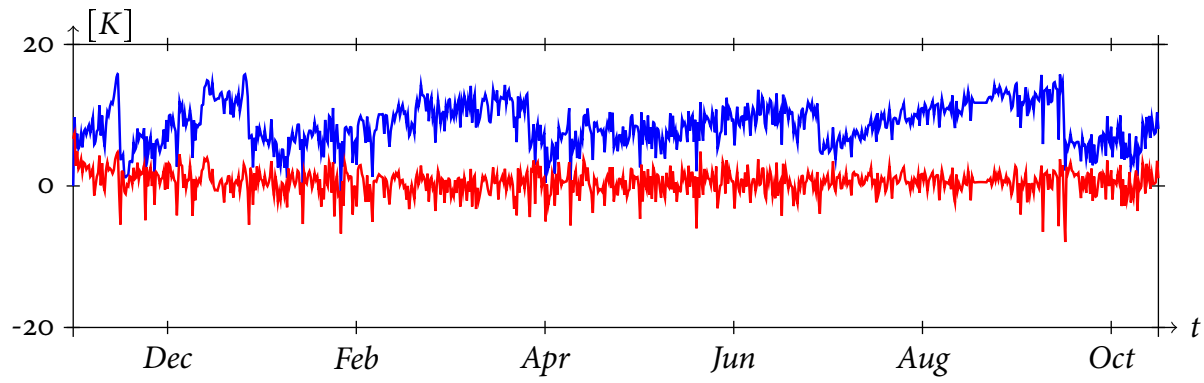

(d) Residual $r_{T_{7}}=y_{T_{7}}-\hat{y}_{T_{7}}$

Figure 7.8: Flame temperature difference $\Delta T_{f}$ (black line) and residuals $r_{i}$ between the CGEKFs which does compensate (red line) and does not compensate (blue line) for the performance degradation in the gas turbine. 


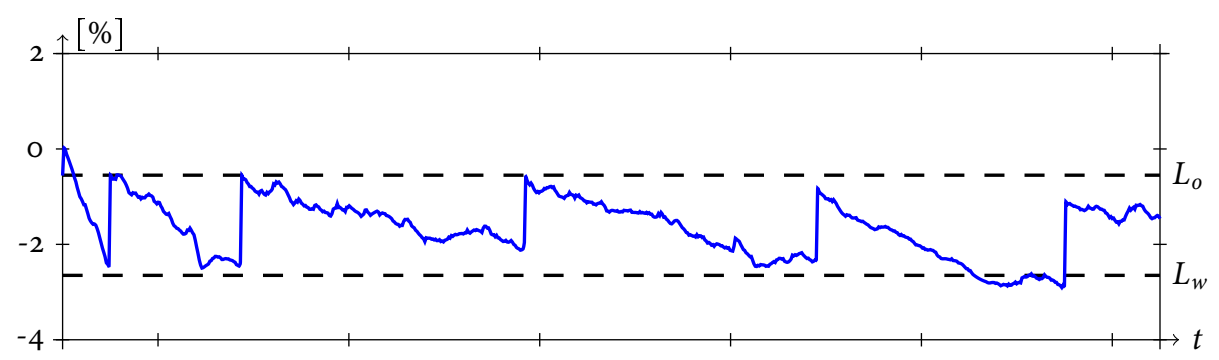

(a) Health parameter $\Delta \eta_{C_{1}}$

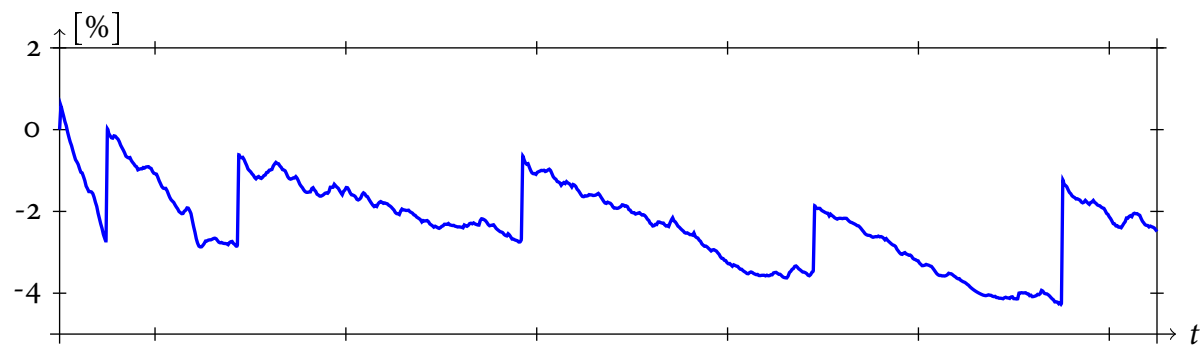

(b) Health parameter $\Delta \Gamma_{C_{1}}$

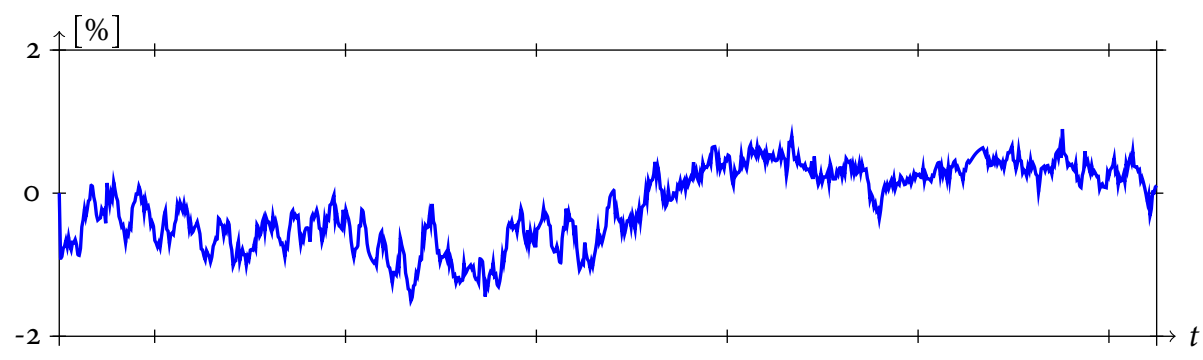

(c) Health parameter $\Delta \eta_{T_{1}}$

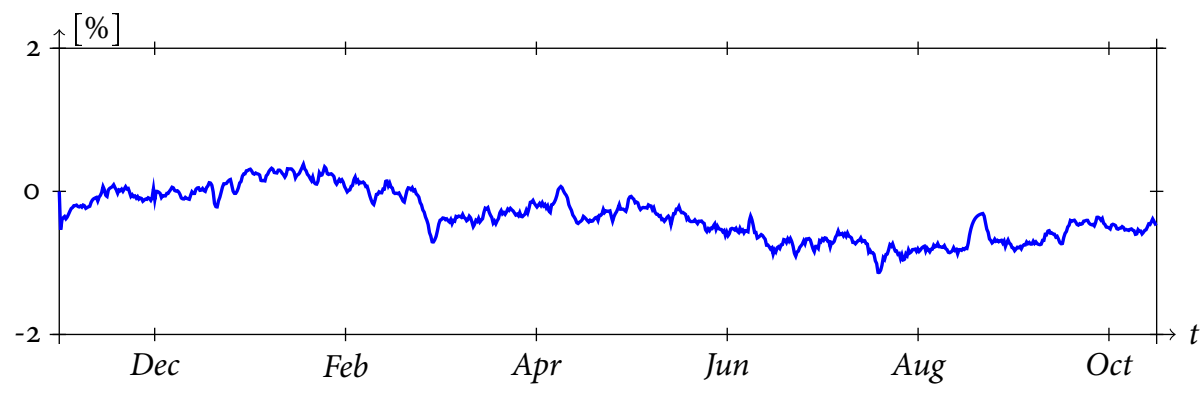

(d) Health parameter $\Delta \Gamma_{T_{1}}$

Figure 7.9: Estimation of the health parameter during a time interval of one year. The degradation in efficiency of $\mathrm{C}_{1}$ can be bounded to an interval of about 2 percent points independent of the atmospheric weather conditions during the year. Thus, it is possible to use a threshold $L_{w}$ to detect when it is time to wash the compressor. The compressor is washed five times: (i) mid November, (ii) end of December, (iii) end of March, (iv) end of June, and (v) mid September. When the compressor wash is performed, the efficiency goes up to $L_{0}$. A degradation in mass flow is shown but it is not so easy to have a static threshold in that case. 
the performance has not dropped enough. Therefore, a simple explanation is that the compressor is washed too early since the time distance from previous compressor wash is too large.

During the winter and summer half year, the ambient conditions such as temperature (Figure B.1) and relative humidity are different. As the $\Delta \eta_{C 1}$ parameter indicates (except for the wash at end of March), it is possible to use the same threshold during the winter and the summer half year to determine if it is time to wash the compressor. The second health parameter $\Delta \Gamma_{C 1}$ is more sensitive to the ambient conditions since the estimation level is different for the winter and summer half year. For the $\Delta \Gamma_{C 1}$ parameter, it is not so obvious to use a static threshold for the compressor fouling detection procedure, but the parameter can anyway be important to supervise and useful in the decision procedure.

For the compressor-turbine T1, the monitored health parameters $\Delta \eta_{T 1}$ and $\Delta \Gamma_{T 1}$ are shown in Figure 7.9(c)-(d). Since these parameters are not varying so much from the nominal baseline and show no visible correlation according to the compressor washes it is assumed that the turbine is healthy. The efficiency of the turbine T1 tends to be little higher on the summer half year than on the winter half year.

\subsubsection{Discussion of the Results in Evaluation 2-3}

One of the objective with the experimental case studies is to see if the CGEKF method gives reliable estimates of the state variables. Since experimental data are considered in Evaluation 2-3, correct values of the state variables are not available. Measurement noise and model uncertainties are always present, therefore the choice of the $Q$ and $R$ matrices affects the state estimates. Since these uncertainties are unknown, the matrices are considered as tuning parameters to obtain reliable state estimates for the present case study. The state estimates considered here is considered to be reliable because: (i) the residuals are centered around the $x$-axis, (ii) no visible trends are seen in the residuals according to the level of compressor fouling, (iii) trends in the residuals disappear when the health parameters are introduced (and the residuals get smaller compared with a case when no health parameters are used see, e.g., Figure 7.8), and (iv) the trend in the estimated performance degradation of the compressor is slow and decreases with time.

\subsubsection{Nonlinear versus Linear Estimator}

An important question is how accurate is the designed nonlinear CGEKF compared to a linear Kalman filter (KF) during different operational conditions (according to the performance chart in Figure 2.3) given the same covariance matrices? To answer that question, a parameter study is performed where a linear Kalman filter (6.33) is designed for the reference ambient conditions at the stationary operation point $50 \mathrm{~Hz}$ $\left(n_{A} \approx 6500[\mathrm{rpm}]\right)$ and $21 \mathrm{MW}$. The two filters have four health parameters each, and evaluated in a number of stationary points at three ambient conditions. The data for the evaluation are generated using the simulation platform in Figure 2.5. The difference between the health parameters at different operating points for the two filters are shown as maps in Figures 7.10-7.12 for the ambient conditions. The evaluation is performed at nominal performance conditions (i.e, no component deteriorations are considered) 
which results in estimations of the health parameters performed by the CGEKF that are zero in all stationary points. Thus, the maps show the estimation error of the KF for the health parameters in percent of the nominal value. In Figure 7.10, the operating range of the gas turbine for the experimental data is shown as grey area in the figures. The difference between the max and min of, e.g., the $\Delta \eta_{C 1}$-parameter is 0.2 percent points and the $\Delta \Gamma_{C 1}$-parameter is 0.8 percent points. The performance deterioration for these two health parameters for a fouled compressor is about 2-3 percent points which was presented in Figure 7.9. When also the ambient conditions change the difference gets larger (see Figures 7.11-7.12).

The evaluation shows that the nonlinear CGEKF is more accurate (the health parameters are zero for all the stationary points) than the linear Kalman filter, but the difference is not so noticeable when the ambient conditions and the turbine speed are nearly constant. When the whole operating area as shown in Figure 2.3 should be covered, the CGEKF is a better choice than the KF. The linear filter is much less time consuming, than the nonlinear filter (seconds instead of minutes). For the industrial gas turbine application, the increase in time for the nonlinear filter is not a problem since application is not time critical. The experimental data is collected for one year which is a long period of time compared to the simulation time.

\subsection{Summary of the Performance Estimation Techniques}

In this section, a summary of the considered performance estimation techniques used for supervision is presented.

\subsubsection{Bell-Mouth Based Estimation}

The first standardized method to detect compressor fouling is based on the bell-mouth pressure drop measurement shown in Figure 7.2(a). The method is simple since the model assumption of the mass flow through the compressor is not so sophisticated. The bell-mouth measurement gives an estimation of the relative mass flow through the compressor. For the available measurement sequence the method indicates poor performance. It is hard to distinguish when a compressor wash is needed, since a baseline for a clean compressor lies in the middle of all points in the diagram. It is desirable that all points in the diagram lie below the baseline and the distance increases with time. A better baseline estimation is received when a physical based model is used to estimate the mass flow as Figure 7.2(d) indicates.

\subsubsection{Measurement Delta}

The second method is based on the so-called measurement deltas. These measurement deltas are more or less, a comparison between an estimated and a measured gas path parameter. A first step is to construct these deltas directly for the measured quantities, i.e., for the pressures, the temperatures, and the shaft speeds along the engine's gas path. The benefits with this method are: (i) it is simple to construct deltas because the 


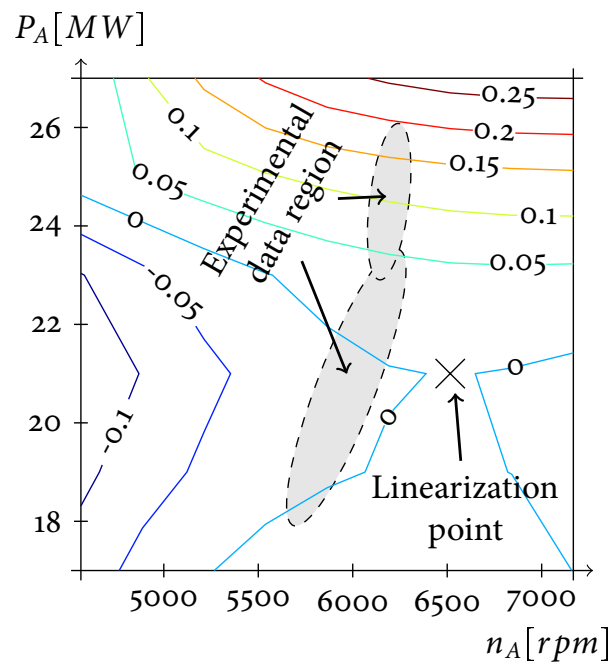

(a) Health parameter: $\Delta \eta_{C 1}[\%]$

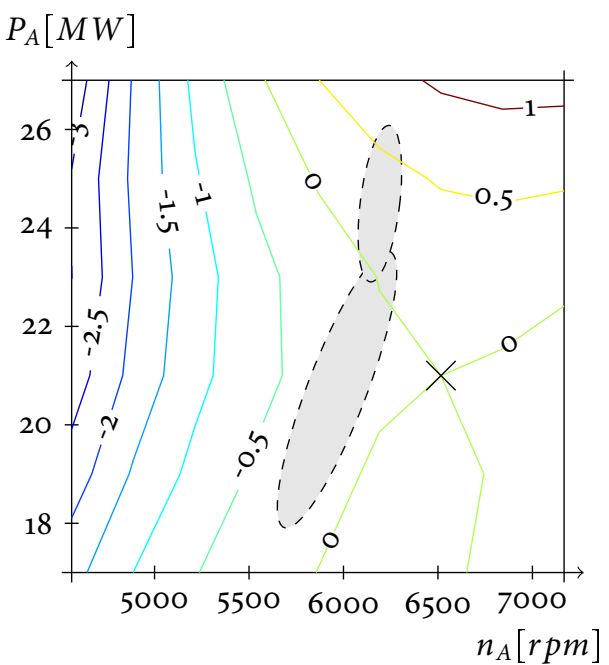

(b) Health parameter: $\Delta \Gamma_{C 1}[\%]$

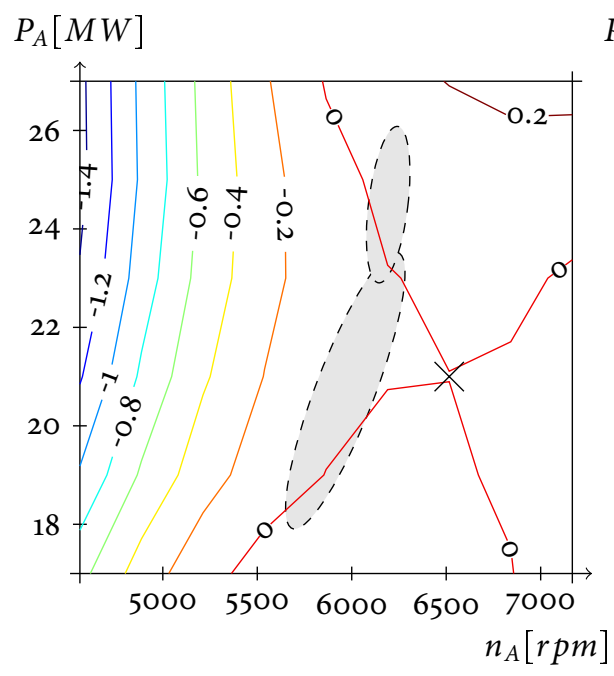

(c) Health parameter: $\Delta \eta_{T 1}[\%]$

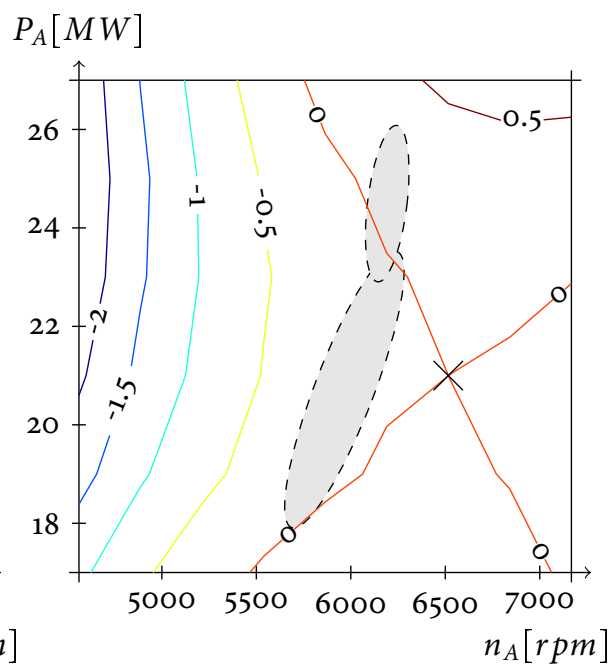

(d) Health parameter: $\Delta \Gamma_{T 1}[\%]$

Figure 7.10: In the figure, the difference in health parameter estimation (in percent of the nominal value) for the nonlinear CGEKF and the linear $\mathrm{KF}$ at the ambient conditions $T=25^{\circ} \mathrm{C}$ and $\varphi=60$ is shown. For stationary conditions, the CGEKF estimate the correct value of the health parameters (which is zero), thus the error for the KF is seen in the figures. The linearization point for the $\mathrm{KF}$ and the region where experimental data are collected are shown in the figure. 


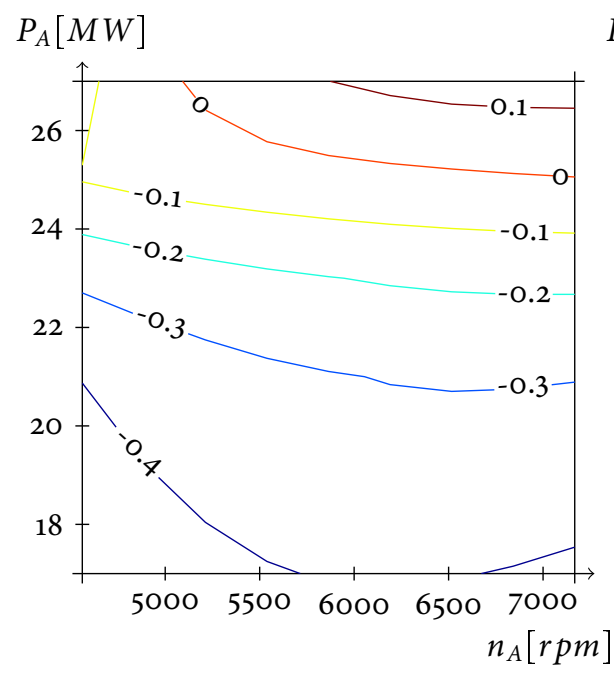

(a) Health parameter: $\Delta \eta_{C_{1}}[\%]$

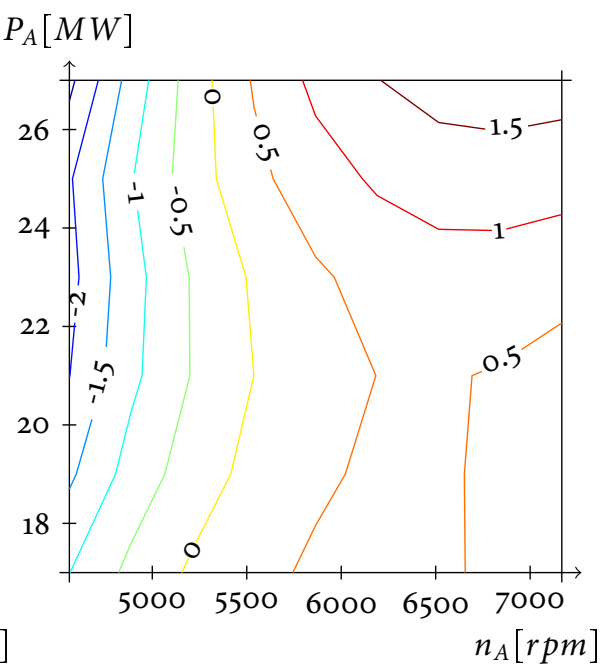

(b) Health parameter: $\Delta \Gamma_{C 1}[\%]$

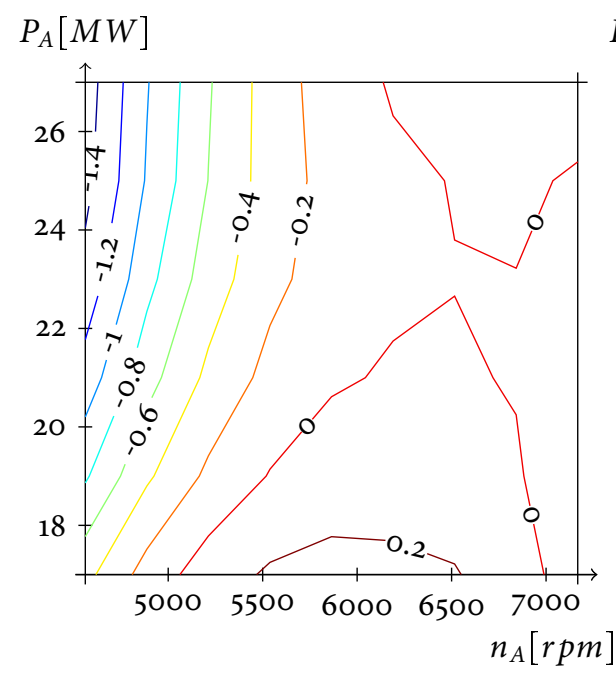

(c) Health parameter: $\Delta \eta_{T 1}[\%]$

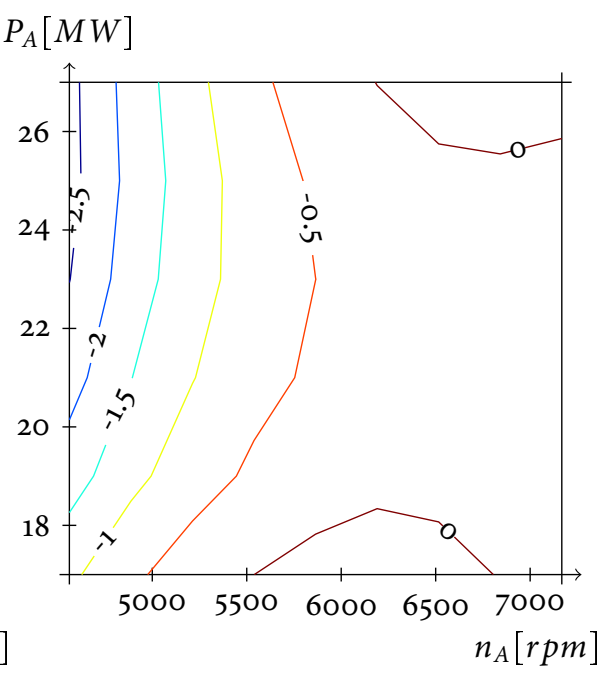

(d) Health parameter: $\Delta \Gamma_{T 1}[\%]$

Figure 7.11: In the figure, the difference in health parameter estimation (in percent of the nominal value) for the nonlinear CGEKF and the linear KF at the ambient conditions $T=15^{\circ} \mathrm{C}$ and $\varphi=40$ is shown. 


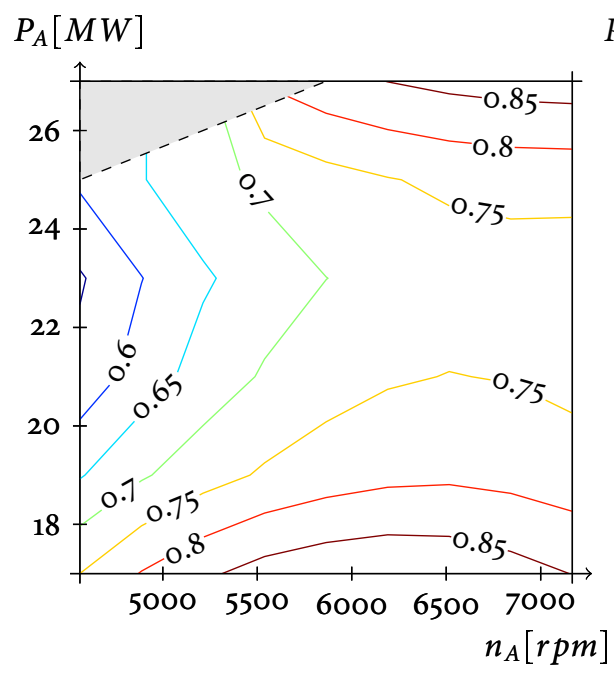

(a) Health parameter: $\Delta \eta_{C 1}[\%]$

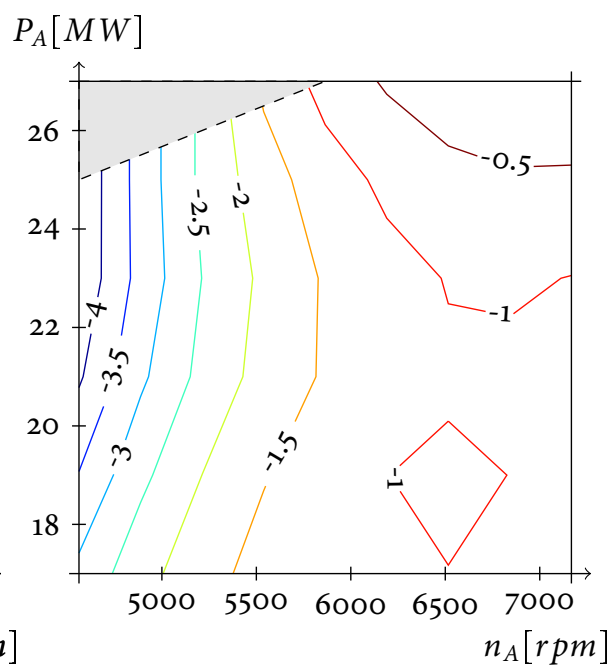

(b) Health parameter: $\Delta \Gamma_{C 1}[\%]$

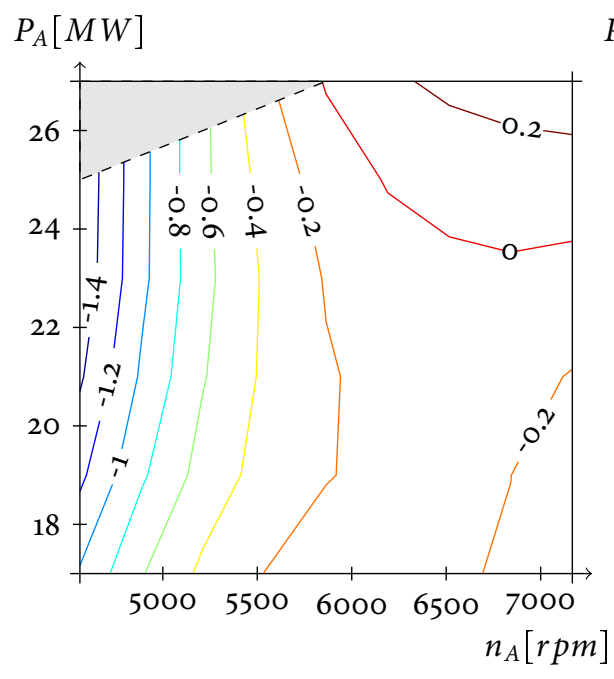

(c) Health parameter: $\Delta \eta_{T 1}[\%]$

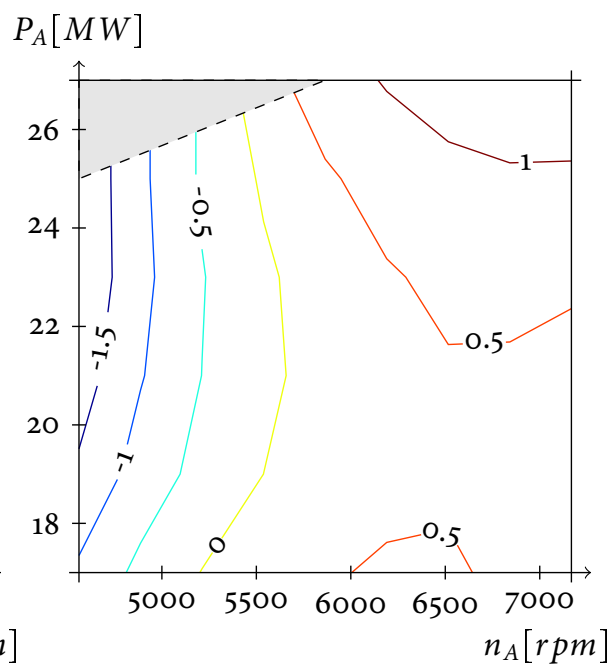

(d) Health parameter: $\Delta \Gamma_{T 1}[\%]$

Figure 7.12: In the figure, the difference in health parameter estimation (in percent of the nominal value) for the nonlinear CGEKF and the linear $\mathrm{KF}$ at the ambient conditions $T=35^{\circ} \mathrm{C}$ and $\varphi=80$ is shown. The grey are are not valid for the given ambient conditions. 
performance model is already available, and (ii) trends due to performance degradation, e.g., compressor fouling, can be detected. The performance model is not augmented with extra parameter or state variables. Therefore, deterioration in components is not modeled explicitly which results in residuals that are dependent on, e.g., compressor fouling. Thus, these residuals may not be suitable for sensor and actuator fault diagnosis since they also capture the performance deterioration. It can also be difficult to associate a number of deltas to a physical fault in the gas turbine. A more sophisticated approach is to use the measurement deltas in the GPA methodology (6.5). Together with the influence matrix $H$ the deviation in performance can be calculated.

The disadvantage with the method is the dependency of the ambient weather condition. The deltas appear to detect trends in performance degradation but also in the ambient atmospheric condition. Therefore it can be difficult to know exactly when a service of the gas turbine is necessary to perform, without further normalization of the data.

\subsubsection{Constant Gain Extended Kalman Filters Constructed Using the Proposed Equation Selection Procedure}

The last method is based on state estimations using a Kalman filter. For this method only relevant parts of the original performance model are considered in the filter which leads to a reduced model in the sense of equation and variables. In an observer, the filtering of signals is encapsulated by the feedback term, so an external filter is not necessary. Benefits with this method are: (i) the modeling is more physical based since health parameters can be injected in the performance equations, (ii) the deviation in, e.g., the efficiency of the compressor is explicitly estimated, (iii) the deviation in efficiency seems to be less dependent of the atmospheric conditions than the measurement delta method, (iv) fault diagnosis can be introduced, and (v) when the framework to generate observers is developed it is possible to generate diagnosis test in a relatively easy manner. Using the designed filter, other state variables, e.g., the combustion flame temperature is estimated. The flame temperature can be utilized in the controller to calculate the turbine inlet temperature. This parameter is important to supervise since too high temperatures in the first blades of the turbine should be avoided.

\subsection{Conclusion}

The topic addressed in the chapter is an evaluation of the designed diagnosis test quantities implemented as constant gain extended Kalman filters (CGEKF). The filter is augmented with health parameters representing efficiency and flow capacity in the compressor and compressor-turbine. The objective with the health parameters is to capture deviation in performance. The CGEKF estimator is evaluated using: (i) data generated from the simulation platform, and (ii) experimental data collected from a gas turbine mechanical drive site. The objectives of the simulation study are to: (i) see if the injected performance deteriorations can be estimated by the designed CGEKF estimator, and (ii) investigate how different ambient conditions affect the estimations. The objectives 
of the experimental case studies are to: (i) estimate the actual health state of the gas turbine over time, (ii) see if the CGEKF gives reliable state estimates, and (iii) check if the CGEKF based concept is suitable to use when the time for a compressor wash is determined.

For the case study which utilized simulated data, the injected performance deteriorations can by estimated by the CGEKF. In the most extreme cases, a change in the ambient condition can be misinterpreted as a performance deterioration in the gas turbine. This can result in, e.g., a compressor wash which is performed to early. The experimental case study is performed using data from a mechanical drive site during a time period of one year. The focus in the study is to evaluate the test quantities used in the FDI-system. The evaluation is performed through: (i) supervision of the performance deterioration, and (ii) estimation of the flame temperature $T_{f}$ which is an important parameter for the controller. The flame temperature is estimated at the same time as the test quantity compensates for the deteriorations in the compressor $\mathrm{C} 1$ and turbine T1. Using the estimated deterioration, a decision of a fouled compressor should be taken. The investigation indicates that it is possible to use the FDI-system to detect a fouled compressor since the deterioration of the compressor decreases about $2 \%$ between the compressor washes. Since the interval and offset of the deterioration are the same independently of the winter or summer period, it is possible to have static thresholds for fouling detection in the FDI-system. Finally, the case study shows that the model design and the proposed systematic test construction procedure can be considered when an FDI-system for an industrial gas turbine is constructed. 



\section{Chapter 8}

\section{Investigation of Fault Diagnosis in the Startup and Shutdown Operating Modes}

During the startup and shutdown sequences of the gas turbine, loads and stresses in components increase. The gain in stress and the fact that the engine goes through many operating points can result in component failures which are triggered. These component failures are, e.g., leakages in valves or increased friction in the mechanical parts. Thus, a good idea is to use the startup and shutdown sequences to detect and isolate faults in gas turbine components using filters. Since the engine is running at different operating modes, the potential of a nonlinear Kalman filter can fully be utilized. The advantage with using filters for fault estimation in nonlinear systems is that parameters can be added easily in the model. These parameters represent the fault which can be estimated by the filter.

\subsection{Background}

The startup and shutdown dynamics of the gas turbine are much faster compared to the performance deterioration according to, e.g., fouling. In Chapter 7 the performance deteriorations are estimated using health parameters. Here, it can be assumed that the health parameters are constant during the startup and shutdown sequences and they can therefore be removed from the diagnosis model. When the health parameters are removed, it is possible to introduce parameters representing fault in components. Another aspect is that different equations are valid during different running phases, i.e., the model consists of hybrid parts which are specified using if-statements. Hybrid models are not directly handled by the developed framework for the diagnosis test generation. Instead, the user needs to specify, e.g., if the combustion is activated, or if the load is applied. Thus, the sequence for the startup to shutdown running mission is divided into the running phases: (1) apply starter motor, (2) ignite combustor, (3) ramp 
up engine, (4) apply load, (5) maintain operating speed, and (6) ramp down engine. These running phases are shown in Figure 8.2 with a subset of gas path parameters. As the figure indicates, the running phases are accomplished sequential for the startup to shutdown running mission.

\subsection{Test Construction Procedure}

The test construction procedure is as follows: (i) design a diagnosis model, (ii) generate an observer for each running phase, and (iii) collect these observers in a test quantity.

\subsubsection{Fault Modeling}

For the fault modeling, parameters which represent the faulty behavior in the startup and shutdown operating modes are introduced in the diagnosis model. The parameter which represents the faulty behavior is later on estimated when the test quantity is constructed according to the methodology described in Chapter 6. In this section, an overview of the modeled faults and in which equations they are introduced are presented. The faults are modeled in the same way as the health parameters were modeled in Chapter 7. Here, the considered fault modes are: (i) leakage in the bleed valve 3 of the compressor, (ii) increased friction in the bearing of the gas generator, and (iii) increased friction in the bearing of the power turbine which all are introduced in the diagnosis model. The faults are added to the diagnosis model using a component based approach, i.e., a component which represents the fault is added using the graphical interface. The fault component is then recognized automatically by the developed parsers.

\section{Component leakage}

A leakage is modeled using a fault parameter which represents the leakage mass flow of the component. This unknown mass flow (which is included in the fault component) is added to the connection point in the gas path where the leakage fault component is placed (i.e., where the leakage should be modeled). In the connection point, the leakage mass flow is written:

$$
\begin{aligned}
m_{\text {flow,leak }} & =f_{\text {leak }} \\
H_{\text {flow,leak }} & =m_{\text {flow, leak }} h
\end{aligned}
$$

where $h$ is the enthalpy and $H_{\text {flow, leak }}$ is the enthalpy flow in the connection point. The constraint:

$$
\dot{f}_{\text {leak }}=0
$$

is added to the fault model. Later on, when the constant gain extended Kalman filter (CGEKF) is designed, it is assumed that this equation has a high level of uncertainty. Thus, the response for the fault parameter in the CGEKF gets fast. 


\section{Increased friction}

A fault in the mechanical bearings is described using a variable which adjust the resistance when the shaft rotates. The increased resistance is modeled as a change in the frictional constant $k_{\text {mech }}$ and is written:

$$
P_{\text {fric }}=f_{\text {fric }} \alpha(n)
$$

where $f_{\text {fric }}$ represents the change in friction, $P_{\text {fric }}$ is the generated power, and $\alpha(n)$ is a function of the shaft speed $n$. In the same way as for the component leakage, the constraint:

$$
\dot{f}_{\text {fric }}=0
$$

is added to the fault model.

\subsubsection{Diagnosis Model}

The diagnosis gas turbine model, with the added faults, has the general differential algebraic equation (DAE) form:

$$
\begin{aligned}
F(\dot{z}, z, u) & =0 \\
y & =h(z) \\
z & =(x, f)^{T}
\end{aligned}
$$

where $f$ represents the unknown faults, $x$ consists of the unknown variables, $y$ consists of the known measurement signals, $u$ consists of the known input signals. The functions $F$ and $h$ together with their arguments are vector valued functions with appropriate dimensions. The modeled faults are here:

$$
f=\left(f_{\text {leak }}^{B V 3}, f_{\text {fric }}^{\text {shaft }}, f_{\text {fric }}^{\text {shaf } t_{0}}\right)
$$

which represent: (i) a leakage in the high pressure bleed valve of the compressor, (ii) an increased friction in the gas generator, and (iii) an increased friction in the power turbine.

\subsubsection{Test Quantity}

During the startup to shutdown running mission, different equations of the diagnosis model are valid according to the switching between the six running phases as shown in Figure 8.2. Therefore, the test quantity for the startup and shutdown sequence consists of four constant CGEKFs which utilize different part of the diagnosis model and have different feedback gains. These four filters are used for the running phases (see index in Figure 8.2): (i) apply starter (1), and ramp down engine (6), (ii) ignite burner (2), (iii) ramp up engine (3), and (iv) apply load (4), and operating speed (5). The filters are generated using the test quantity generation procedure described in Section 7.4. The $Q$ and $R$ matrices are tuned in that way so the response of the state variables representing the faults are fast. The filters are generated from the same diagnosis model (except for case (5) when health parameters are considered). Between the running phases, the estimated state variables are saved and used as initial conditions to the next phase. The running 
phase when the engine has reached the operating speed condition (5), the test quantity developed in Chapter 7 (which includes health parameters) can be used. The value of the health parameters from the previous operating speed condition can be used as initial values for the health parameters. The other filters can be adjusted with the previous value of the health parameters, i.e., normalize the performance. The method for sensor and actuator fault diagnosis, which is introduced in Chapter 9 can also be combined with the diagnosis of the component faults in the startup and shutdown phases. An overview of which faults that can be diagnosed during the various running phases is shown in Figure 8.1.

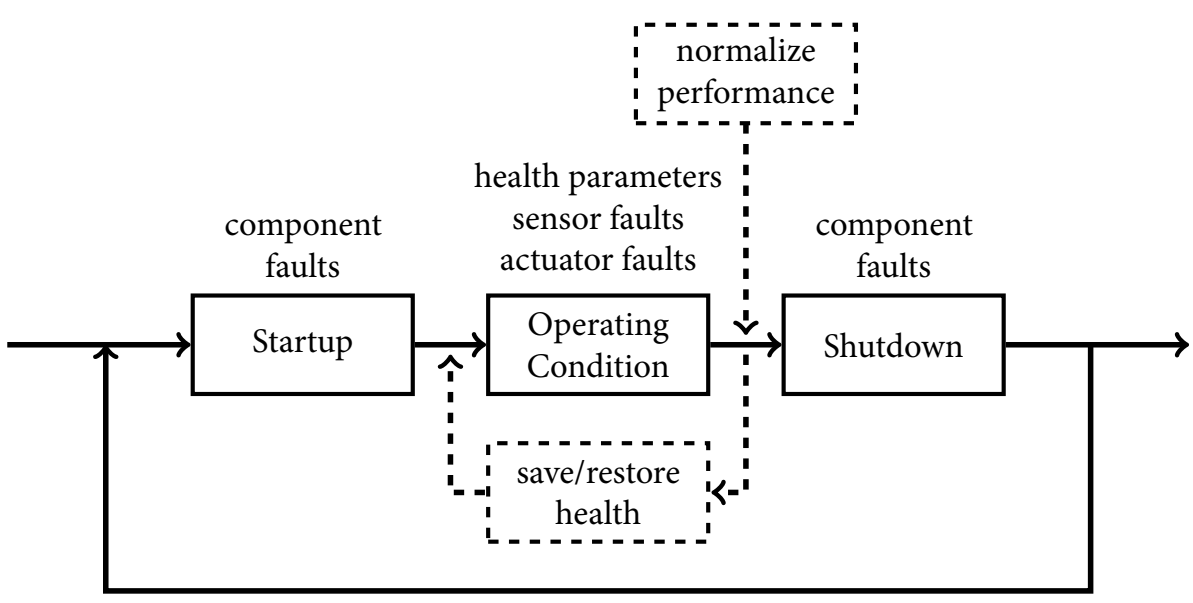

Figure 8.1: An overview of the possible type of diagnosable faults during the various running phases. When the engine starts the shutdown phase, the performance is normalized with the values of the health parameters.

\subsection{Simulation Study}

The designed test quantity is evaluated using simulated data gathered from the simulation platform shown in Figure 2.5. The component faults are injected in the simulation platform during the ramp up engine phase (3). The faults are described as: (i) an outgoing mass flow in the bleed valve 3 position of $3 \%$ of the nominal mass flow through the compressor, and (ii) an increased friction of $250 \%$ of the nominal friction coefficients in the shafts of the gas generator and the power turbine. Since the nominal values of the friction coefficients are small, the values of the injected faults get large in percent. Smaller values of the faults in the friction are possible to diagnose. The problem is rather that large faults are difficult to investigate since the simulation platform cannot handle too large faults. An injected fault which is too large results in: (i) many state events, (ii) a stiff system, and (iii) a simulation that crash. During the simulation, it is assumed that 
the performance is at nominal reference conditions and no noise is added to the output signals.

\subsubsection{Fault Free Sequence}

For the fault free sequence, the objective is to compare some of the estimated variables with the corresponding variables in simulated data. Important variables (which are not measured) to supervise are, e.g., the mass flow of air through the compressor, and the combustion flame temperature $T_{f}$. These two variables are shown in Figure 8.2 together with the rotational speed of the gas generator and the power turbine (which are measured). The startup and shutdown sequence is divided into the six phases: (1) apply starter, (2) ignite burner, (3) ramp up engine, (4) apply load, (5) operating speed, and (6) ramp down engine (release load) which is marked in the figure.

The result of the fault free investigation is a test quantity which produces estimations which agree with the simulated data. The residuals and fault parameters are zero except during some time in the beginning of each phase. This phenomenon is seen in, e.g., the fault parameters in Figure 8.4 or Figure 8.3 in the beginning of phase 3 where it takes some time before the parameters go back to zero.

\subsubsection{Component Faults - Leakage in the Compressor and Increased Friction in Mechanical Bearings}

For the simulation cases where component faults are introduced, the objective is to estimate the faults correctly with the diagnosis test. How fast the test responds to the fault depends on how the covariance matrices $Q$ and $R$ are chosen when the CGEKF is designed. The tradeoff is between how much the estimations should rely on the model and on the measurements. Thus, the elements in $Q$ which belong to the fault parameters are associated with a large value, i.e., the fault estimations rely more on the measurements than on the model. When the fault is estimated correctly by the test quantity, the fault is decoupled and the test quantity can be used to supervise the same variables as for the fault free case. The considered faults are: (i) a leakage in the bleed valve 3 with an amplitude of $3 \%$ of the nominal mass flow through the compressor, (ii) an increased friction coefficient in the bearing of the gas generator with an amplitude of $250 \%$ of the nominal friction coefficients, and (iii) an increased friction coefficient in the bearing of the power turbine with an amplitude of $250 \%$ of the nominal friction coefficients. For each fault mode, a measurement sequence is generated where the actual fault is injected. The fault is injected when the engine ramp up, i.e., during section 3 in Figure 8.3.

All three fault cases can be diagnosed by the developed test quantity. The supervised variables (e.g., mass flows and temperatures) agree with the corresponding variables in the simulated data which is not the case if no fault estimation parameters are used in the CGEKFs. In Figure 8.3 and Figure 8.4, the fault case of the leakage in the compressor and the increased friction in the gas generator are shown. As shown in the figures, it seems that the fault parameters which are sensitive to the injected faults can estimate the fault correctly and be used to trigger an alarm. The fault parameters which should not be sensitive to the injected faults are not reacting. 


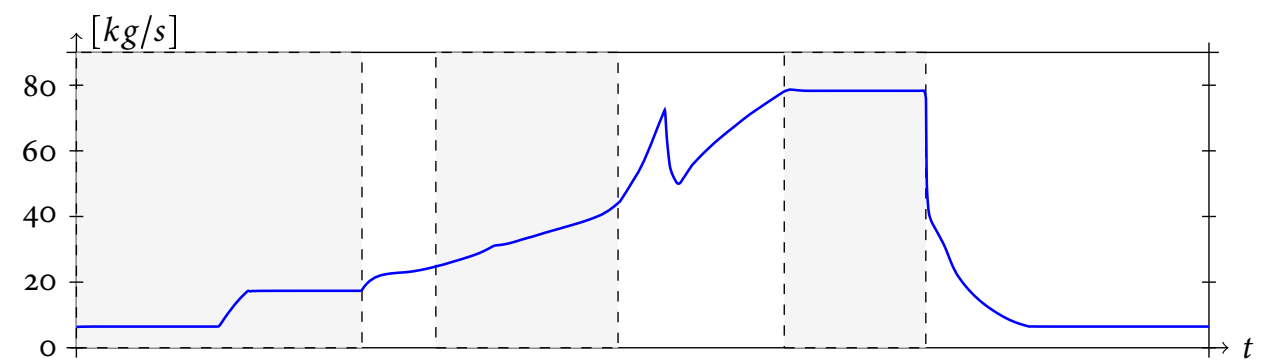

(a) Mass flow of air trough compressor C1.

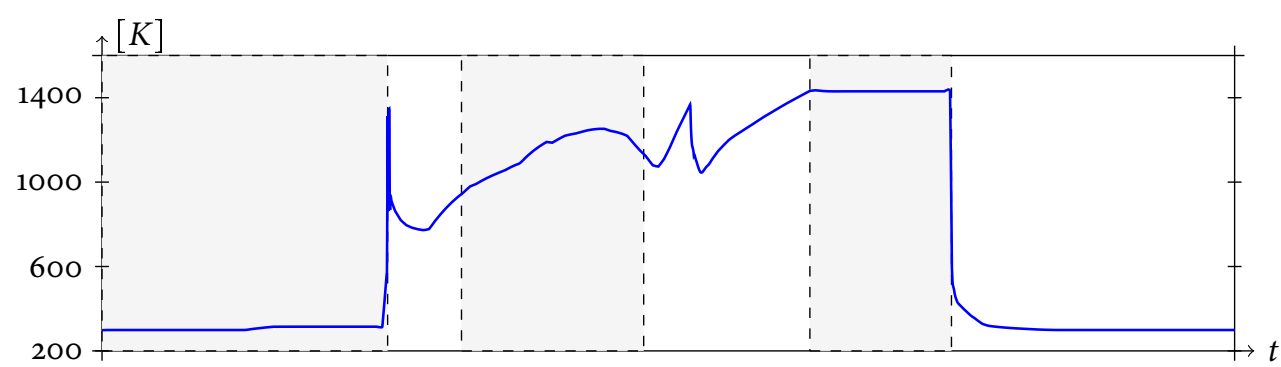

(b) Flame temperature $T_{f}$.

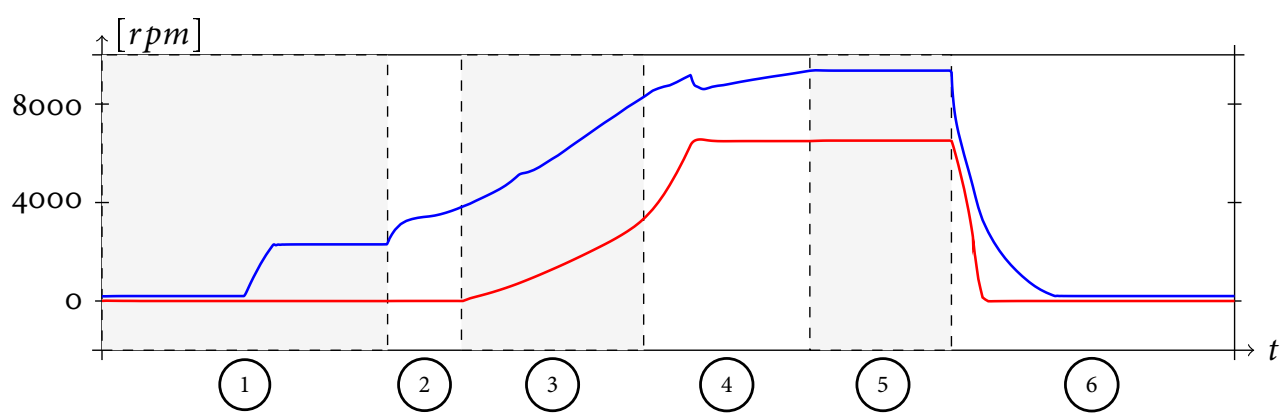

(c) Speed of gas generator (blue), and speed of power turbine (red).

Figure 8.2: Typical appearances of a startup and shutdown sequence. The sequence consists of the phases: (1) apply starter, (2) ignite burner, (3) ramp up engine, (4) apply load, (5) operating speed, and (6) ramp down engine (release load). 


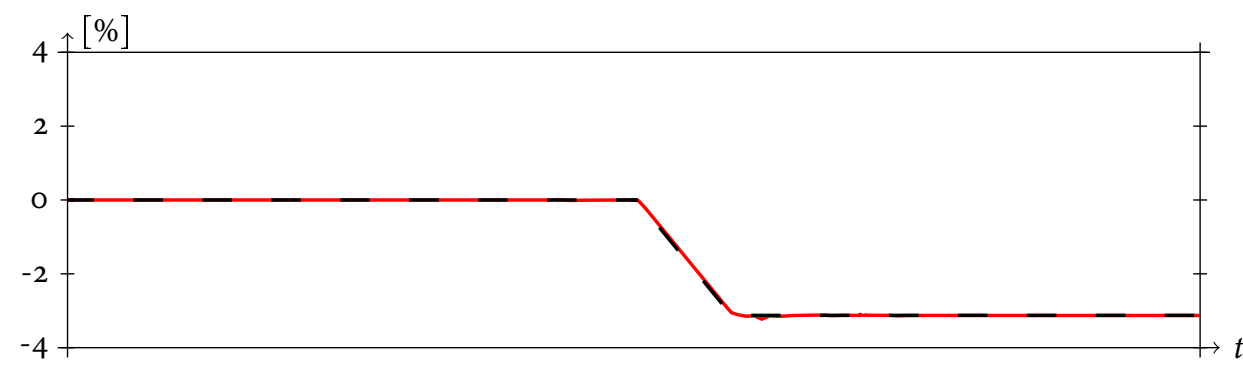

(a) Estimation parameter of leakage in bleed valve 3 of compressor (red), and injected fault (black).

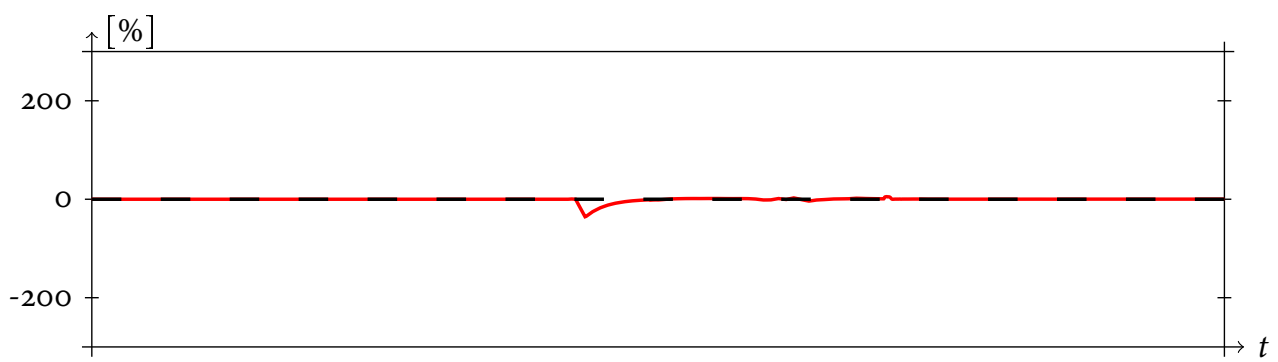

(b) Estimation parameter of increased resistance in the bearing of gas generator (red), and injected fault (black).

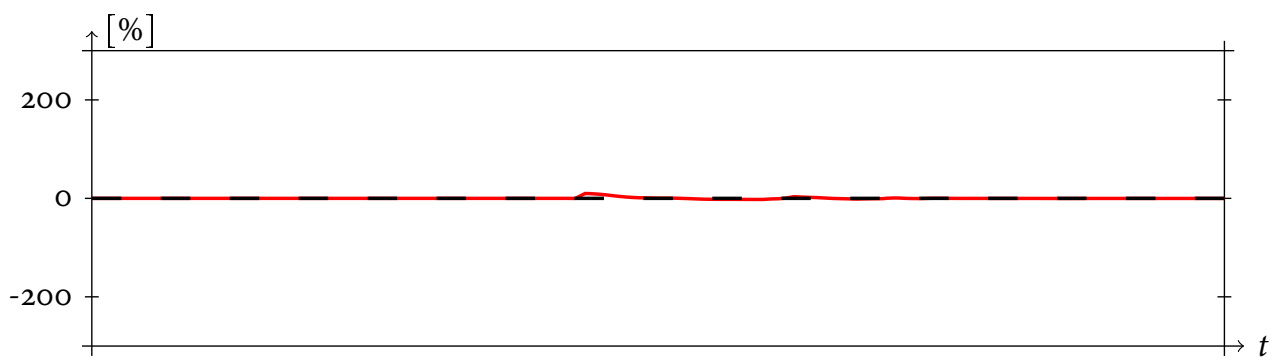

(c) Estimation parameter of increased resistance in the bearing of power turbine (red), and injected fault (black).

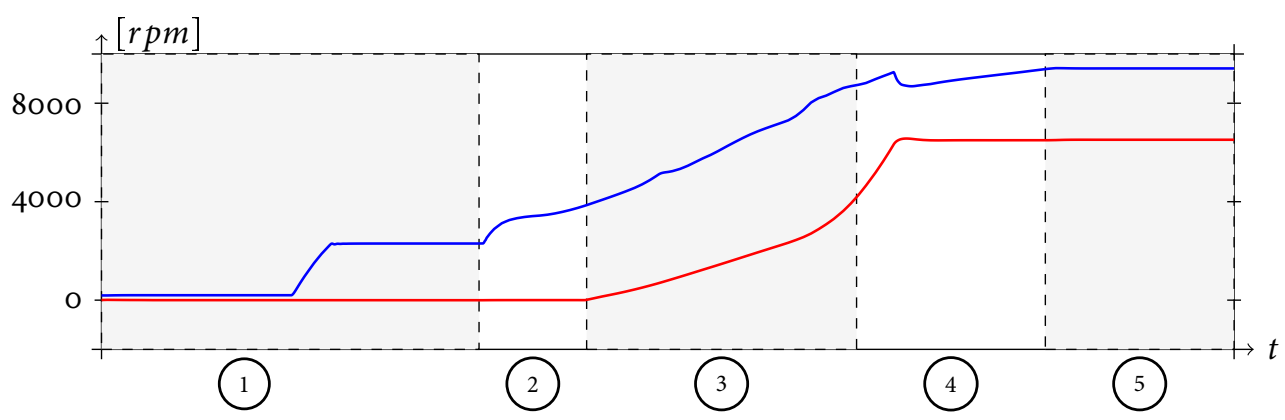

(d) Speed of gas generator (blue), and speed of power turbine (red).

Figure 8.3: Fault case - leakage in bleed valve 3 of compressor C1. 


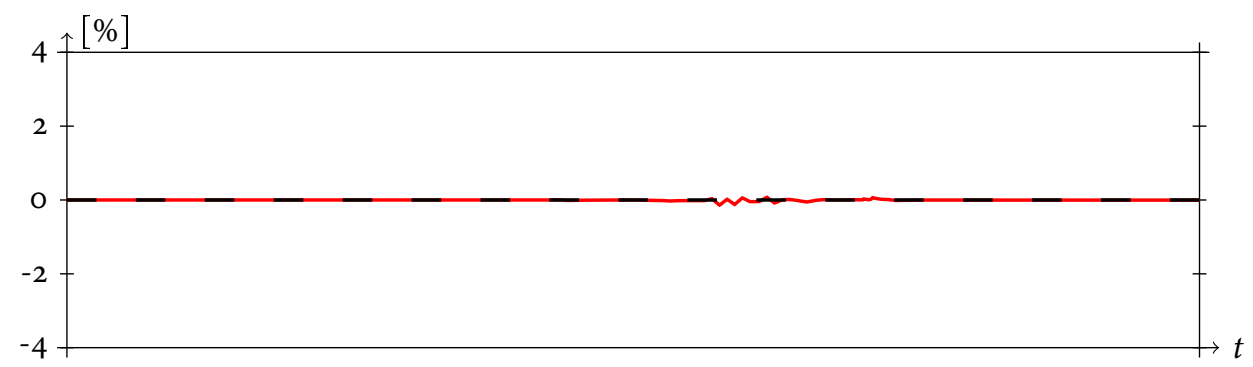

(a) Estimation parameter of leakage in bleed valve 3 of compressor (red), and injected fault (black).

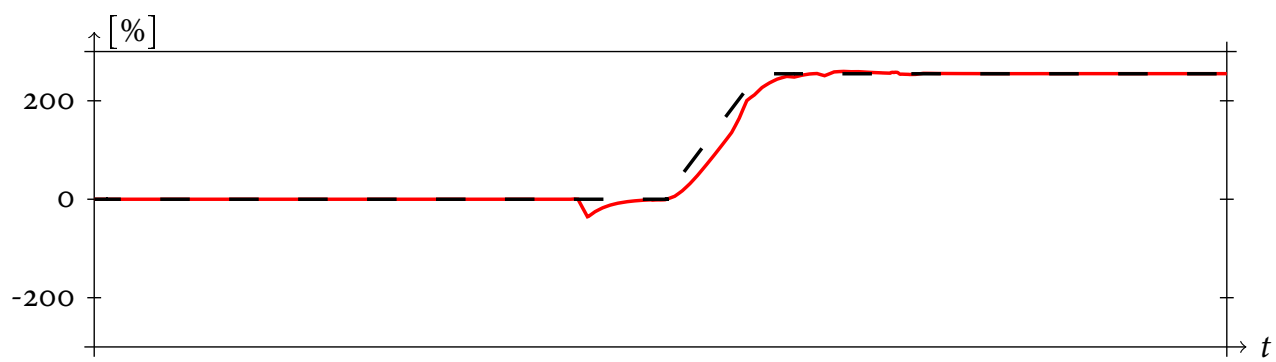

(b) Estimation parameter of increased resistance in the bearing of gas generator (red), and injected fault (black).

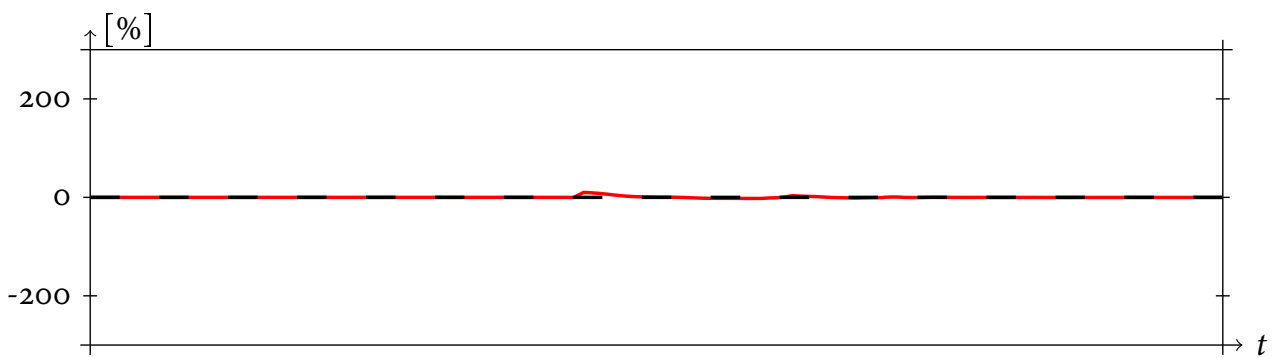

(c) Estimation parameter of increased resistance in the bearing of power turbine (red), and injected fault (black).

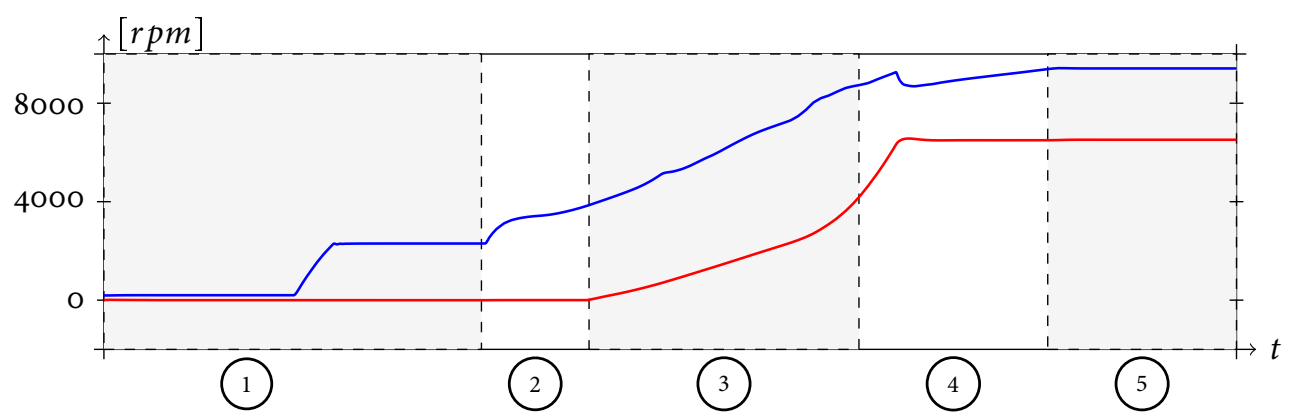

(d) Speed of gas generator (blue), and speed of power turbine (red).

Figure 8.4: Fault case - increased resistance in ball bearing of gas generator. 


\subsection{Conclusion}

In the chapter, a pilot study is presented where the objective is to investigate and develop a diagnosis test quantity used for detection and isolation of component faults in the startup and shutdown sequences of the gas turbine application. Since the engine goes through many operating modes in this case, the potential of the nonlinear Kalman filter can fully be utilized. The startup and shutdown sequences can be relevant to study for diagnosis purposes since the engine goes through many operating modes which can trigger faults which are not seen during, e.g., stationary conditions. The focus in the study is to demonstrate the ability to introduce parameters in the Kalman filters which are used to estimate fault in components. In this study, three different faults are investigated: (i) a leakage in the bleed valve 3 of the compressor, (ii) an increased rotational friction coefficient in the gas generator, and (iii) an increased rotational friction coefficient in the power turbine. These three faults are possible to detect and isolate using data from the simulation platform. Here, the three faults are introduced in the same Kalman filter, but other fault isolation configurations and fault modes are also possible to obtain. 



\title{
Chapter 9
}

\section{Diagnosis and Fault Tolerant Supervision of Industrial Gas Turbines}

\author{
Diagnosis and Fault Tolerant Supervision of Industrial Gas Turbines* \\ Emil Larsson, Jan Åslund, Erik Frisk, and Lars Eriksson \\ Vehicular Systems, Department of Electrical Engineering. \\ Linköping University, SE-581 83 Linköping, Sweden.
}

\begin{abstract}
Monitoring of industrial gas turbines is important since it gives valuable information of the process health, and component failures. Sensor and actuator faults affect the engine's operation point and complicate the determination of compressor wash intervals. Thus, it is important to have a fault detection and isolation (FDI) system which can detect and isolate these faults at an early stage. In the paper, an approach to construct an FDI-system which utilizes multiple constant gain extended Kalman filters is suggested. Each filter decouples one of the considered faults, and in case of a fault, it is only one of the filters that are consistent with the input and output signals. The filter which decouple the fault is used to estimate the performance, thus a fault tolerant system for supervision and control is obtained. To evaluate the FDI-system, two case studies are performed based on: (i) simulated data, and (ii) experimental data from a gas turbine site. The investigation shows that all important faults are detectable and isolable, at the same time the performance is supervised. The estimation of performance can be used for control or to determine when it is time to wash the compressor.
\end{abstract}

* This chapter is an edited version of the paper Diagnosis and Fault Tolerant Supervision of Industrial Gas Turbines which is submitted for journal publication. 


\subsection{Introduction}

In industrial gas turbines, deterioration in components throughout the gas path is commonly occurring and contributes to the overall performance deterioration of the engine. To maintain reliable engine operations it is crucial to detect and isolate faults in sensor and actuator signals throughout the gas path. It is important to discriminate between an instrumentation fault and a component deterioration to avoid unnecessary operation stops. Instrumentation faults which are left undetected can result in wrong input signals to the controller. An important signal, used in the controller, is the combustion flame temperature which is not measured by any sensors but is estimated in the controller using the instrumentation signals together with a simplified engine model. The flame temperature estimate is affected by: (i) the component deteriorations, and (ii) the faults in sensors and actuators. Thus, both these aspects need to be considered in the fault detection and isolation (FDI) system and in the controller to maintain reliable engine operations. An undetected fault can also result in a misclassification of a fouled compressor which increases the environment impact, the fuel consumption, and a component failure in the worst case scenario. When a single instrumentation fault is present in the engine, it is preferably that the operation can continue in a robust manner without interruption until the next overhaul, which results in a more efficient planning of service and maintenance. The focus herein is on the FDI-part, but the state variables estimated by the FDI-system can with great advantage also be used in the controller.

One of the major contributions which cause performance deterioration in industrial gas turbines is fouling (Diakunchak, 1992; Kurz et al., 2009). Fouling is caused by small particles and contaminants in the air caught by the compressor blades which reduces the efficiency and mass flow of the compressor. A common approach in the gas turbine diagnosis literature, to estimate component deterioration is to use filters combined with health parameters (Luppold et al., 1989; Volponi et al., 2003). The filters are often Kalman based, and the health parameters are correction factors for, e.g., efficiencies and flow capacities in the components. In Kobayashi et al. (2005), a constant gain extended Kalman filter (CGEKF) is investigated where a nonlinear model can be combined with the offline calculation of the Kalman gain matrix. It is a good idea to use the health parameters to decide the level of compressor fouling, i.e., to determine when it is time to wash the compressor which was investigated in Larsson et al. (2014a).

In Merrill et al. (1988); Kobayashi and Simon (2005), a fault isolation strategy based on hypothesis testing is presented where a hypothesis is specified for each fault case. The tests consist of linear filters and when a fault is present in the system, only one hypothesis is valid. Therefore, it is possible to isolate the faults. When the test compensates for the component deterioration, the residuals become less dependent on the deterioration level which results in a possible usage of smaller threshold for fault detection. In Merrill et al. (1988), no compensation is made for the component deterioration, and in Kobayashi and Simon (2005) the component deterioration is compensated offline for the sensor fault hypotheses. In Kobayashi and Simon (2003), the compensation of deterioration in components is performed online for all fault hypothesis using only health parameters. Since the health parameters try to explain the faulty sensor or actuator value, the detection and isolation performance may be weaker than in Kobayashi and Simon (2005). Since 
noise is present in the measurement sequences, this results in test quantities which may not alarm although they are sensitive to the considered fault. Especially slowly drifting sensor and actuator bias faults can be difficult to detect with the proposed method. To get better detection performance, an idea is to estimate the fault instead of removing one sensor equation in each test quantity. This leads to the case where the correct fault hypothesis always estimates the fault, and the estimated fault can be used for fault detection in the FDI-system. The disadvantage is that all tests are sensitive to all considered faults, thus ideally it is not possible to isolate the fault. In practice, it can be assumed that the test quantity which estimates the fault also decouple the fault in the test quantity when the drifting bias is slower than the dynamic of the estimation parameter in the test, i.e., slowly drifting faults are easier to decouple than abrupt faults in that sense.

To gain the overall diagnosis performance, i.e., increase the fault detectability and reduce the false alarm probability, it is desirably to have an FDI-system which consists of diagnosis tests based on models with high accuracy. When a model, e.g., consists of not negligible biases, a high frequency rate of false alarms can be generated. Thus, a reduction in false alarms can reduce the ability to detect a fault since the thresholds can be lowered. In the developed FDI-system, the tests quantities are based on nonlinear models which are presented in Larsson et al. (2014a). These nonlinear models are used for performance analysis by Siemens Industrial Turbomachinery AB (SIT). In Larsson et al. (2014a), a framework to automatically generate CGEKF based estimators from the physical based nonlinear gas turbine model implemented in Modelica (Modelica Association, 2007) is also presented. An automatic and systematic design method is valuable since the model is a large differential algebraic equation (DAE) model with nonlinear parts and the design of the filters might be a complex task without an automatic method. For the work presented herein, experimental data and a simulation platform are provided by SIT. Using the simulation platform, data for the FDI-system can be generated under various operational conditions.

\subsubsection{Problem Statement}

The objective of this work is to design and evaluate an FDI-system for an industrial gas turbine based on hypothesis testing, while so many health parameters as possible are utilized in the diagnosis test quantities. The FDI-system should diagnose single sensor or actuator faults at the same time the supervision of performance is maintained, i.e., an FDI-system used for fault tolerant supervision. The focus here is on detection and isolation of slowly drifting bias faults. When a fault is detected and then isolated, the input signals used in, e.g., the controller should be based on estimates where the fault and the component deteriorations are decoupled. For each fault mode, a test quantity has to be designed carefully as a part of the FDI-system.

The evaluation is performed using: (i) data generated from the simulation platform, and (ii) experimental data collected from a gas turbine site. The health and tuning parameters of the test quantity should be adjusted to work together with the experimental data. For the two evaluation cases, a single sensor or actuator fault is injected in the data sequences for each fault case. The objectives with the two evaluation studies are to: 
(i) examine each test quantity if it gives reasonable values, (ii) check if the considered faults are detectable and isolable, (iii) false alarms should be avoided, (iv) investigate how large faults that are possible to detect and isolate with the designed FDI-system, (v) check if it is possible to isolate the fault before the compressor wash alarm is triggered, (vi) inspect the health parameter estimates for each fault hypothesis and compare the results with the fault free estimates, and (vii) use the estimated health parameters to decide if it is time to wash the compressor or not.

\subsubsection{Outline and Contributions}

The paper starts with the design procedure of the physical based estimators (Sec. 9.2), and continues with the diagnosis test quantity construction (Sec. 9.3). In Sec. 9.4, the description of the fault isolation method which is used in the FDI-system is presented. The next step is to evaluate the FDI-system using data gathered from the simulation platform (Sec. 9.5), and continues with an investigation of an experimental case study (Sec. 9.6). In the two evaluations, faults are injected into the measurement signals.

The first contribution of the paper is a systematic design procedure of an FDI-system, for an industrial gas turbine, which can be used for fault tolerant supervision and control when a single sensor or actuator fault is present. The diagnosis test quantities in the FDI-system are based on nonlinear differential algebraic equation (DAE) models. The corresponding models at SIT are used for performance analysis. It is shown that the proposed method gives good fault detection performance, also for slowly drifting sensor and actuator bias faults. To increase the fault isolation performance, an upper and lower limit of the health parameters is introduced.

The FDI-system is applied to an evaluation of: (i) simulated data, and (ii) experimental data from a gas turbine site. For the two case studies, all considered fault modes are detectable and the most of them are isolable. When a unique diagnosis is determined by the FDI-system, the performance can be supervised using the test quantity which are based on the most probably fault hypothesis. The deviation in performance of the compressor can then be used to determine if it is time to wash the compressor, also when a single sensor or actuator fault is present.

\subsection{Gas Turbine Diagnosis Modeling}

The objective in this section is to describe the gas turbine models that form the basis of the developed FDI-system. Given these models, diagnosis test quantities $T_{i}$ can be generated which are the fundamental part of the FDI-system. The systematic and automatic test design procedure is presented in Larsson et al. (2014a). In the design, the nonlinear gas turbine model is transformed to a test quantity which is implemented as a constant gain extended Kalman filter (CGEKF). The filter is then used to supervise the performance due to, e.g., compressor fouling and control purposes. Here, the focus is on fault isolation, thus the FDI-system consists of more than one test quantity. The FDI-system can supervise the performance at the same time as a single fault is present. 
For each estimator in the FDI-system, the main design choices are to decide: (i) the number, and (ii) the position of the health parameters, at the same time one fault is decoupled in the diagnosis test. The investigation shows, e.g., that it is difficult to decouple a fault in the speed sensor of the gas generator at the same time the deterioration in mass flow through the compressor is supervised. Therefore, the focus in this section is to decide which health parameters that can be modeled and which faults that can be decoupled in each test to have an estimator that can be running using the experimental data without instabilities.

For each test quantity $T_{i}$, a physically based diagnosis model $D_{i}$ is constructed. The diagnosis model is derived from a model which is similar, and validated against a reference model (Idebrant and Näs, 2003) used by SIT for performance analysis. The two model concepts used for diagnosis and performance analysis are first presented in Larsson et al. (2010) and further improved in Larsson et al. (2014a). In the diagnosis model $D_{i}$, concepts related to component degradation and fault diagnosis are introduced which are not considered in the model used for performance analysis. Thus, the number of: (i) component degradation parameters (health parameters), and (ii) sensor or actuator faults, can be specified where each configuration results in a separate diagnosis model. The nominal performance model of the gas turbine has the DAE form:

$$
\begin{aligned}
F(\dot{x}, x, u) & =0 \\
y & =h(x)
\end{aligned}
$$

where $x$ represents the unknown variables, $u$ represents the input signals, and $y$ represents the measurement signals.

\subsubsection{Measurement Signals}

All signals used in the FDI-system are based on measurements, but here the signals are divided into the two groups: (i) actuator signals (input signals), and (ii) sensor signals (output signals). In Fig. 9.1, a schematic view of the gas turbine and its measurement signals are shown.

\section{Input (Actuator) Signals}

The available input signals are divided into two groups. The first group consists of: (i) the pressure $p_{0}$, (ii) the temperature $t_{0}$, and (iii) the relative humidity $\varphi_{0}$ of the ambient air. These signals are assumed to be non-faulty and are used to determine the gas properties of the ambient air. The second group of input signals is: (i) the mass flow of fuel $m_{f}$, and (ii) the power generated by the application $P_{A}$. The signals in the second group can be faulty and should be diagnosed by the FDI-system.

\section{Output (Sensor) Signals}

The available output signals are the measured quantities of: (i) the temperature $t_{2}$ and pressure $p_{1}$ before compressor, (ii) the discharge temperature $t_{3}$ and pressure $p_{3}$ after compressor, (iii) the temperature $t_{7}$ and pressure $p_{8}$ after power-turbine, and (iv) the 


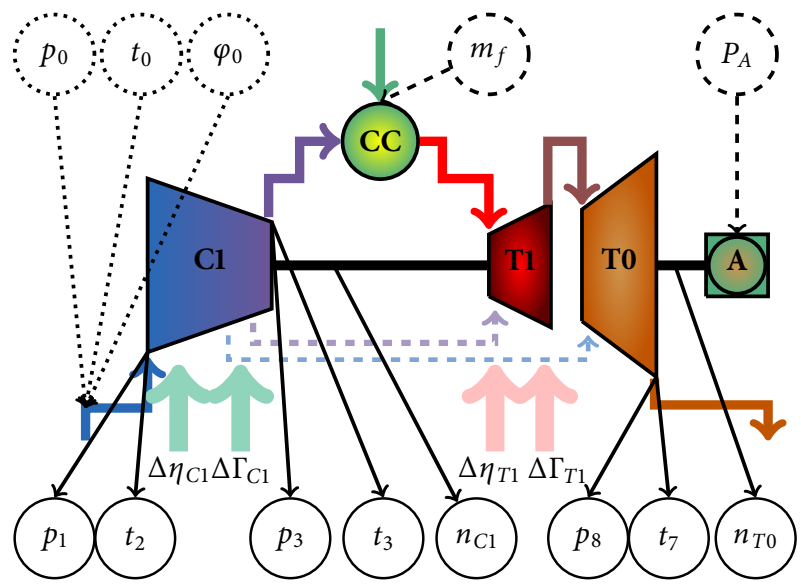

Figure 9.1: The gas turbine with the output signals (solid), the input signals (dashed), the ambient input signals (dotted), and the health parameters $\left(\Delta_{i}\right)$. The secondary air flows, used to cool the first turbine blades, are shown with dashed arrows in the figure.

speed of gas generator $n_{C 1}$ and the power-turbine $n_{T 0}$. The subscript notation in the measured quantity describes at which cross-sectional area the sensor is located. A low number represents the air entrance and a high index number represents the position where the exhaust gas leaves the gas turbine. A challenge with this specific product model is the absence of an instrumentation sensor between the two turbines. The lack of these types of sensors makes the diagnosis and monitoring procedure more difficult to perform. Having ideal thermocouples in that cross-section should reduce the uncertainty of the gas path parameters in the gas generator.

\subsubsection{Health Parameters}

To capture unmeasured performance deterioration in the gas turbine components, a common approach in the field of gas turbine health management and control is to introduce a number of physical based quantities named health parameters (Kobayashi and Simon, 2005; Simon and Simon, 2005; Borguet and Léonard, 2008). These parameters are introduced in the equations where efficiencies and flow capacities are calculated. The parameters are then estimated by, e.g., a Kalman filter. Three main advantages using health parameters appear to be: (i) physical based model concept, (ii) the ability to supervise component deteriorations, e.g., due to fouling, and (iii) remove trends in the residuals due to component deteriorations. When the health parameters are used, two main disadvantages appear and make the diagnosis procedure more difficult: (iv) the observability of the system is affected, and (v) a faulty sensor value may be, in some sense, captured by the health parameters. The maximum number of health parameters, which can be considered in the model due to the observability criteria, is restricted by the number of sensors. Thus, the maximum number of health parameters is equal to the 
number of sensors. In practice, this maximum number of health parameters is much smaller and depends on, e.g., where in the model the health parameters and sensors are located. The disadvantage (v) can result in a test quantity which is sensitive to a specific fault, but does not trigger since the health parameters compensate for the faulty sensor value. Thus, the fault signature is not seen in the residuals since model uncertainties and measurement noises are always present. To reduce this problem and increase the fault isolation ability, an idea is introduce constraints of the health parameters to restrict the allowed interval of deterioration.

In total, there are four health parameters (see Fig. 9.1) to estimate how the components deteriorate. These parameters are added additive in the performance characteristic equations, and for the compressor $\mathrm{Cl}$ these parameters are introduced to estimate deterioration in: (i) the isentropic efficiency $\Delta \eta_{C 1}$, and (ii) the mass flow of air $\Delta \Gamma_{C 1}$. In the compressor-turbine $\mathrm{Tl}$, two health parameters are introduced to estimate deterioration in: (iii) isentropic efficiency $\Delta \eta_{T 1}$, and (iv) turbine flow capacity $\Delta \Gamma_{T 1}$.

In the DAE form, the model which taking into account the component deterioration is written:

$$
\begin{aligned}
F(\dot{x}, x, \Delta, u) & =0 \\
\dot{\Delta} & =0 \\
y & =h(x)
\end{aligned}
$$

where $\Delta=\left(\Delta \eta_{C 1}, \Delta \Gamma_{C 1}, \Delta \eta_{T 1}, \Delta \Gamma_{T 1}\right)^{T}$, and $x$ denotes the unknown variables. Since the component deterioration is slow relative to other gas turbine dynamics, the derivative is considered to be zeros. Thus, the constraints in Eq. (9.2b) are added to the model.

\subsubsection{Sensor and Actuator Faults}

Slow drifting bias faults can be difficult to detect according to the methods presented in Kobayashi and Simon (2003); Larsson et al. (2011). This depends on a fault signature in the residuals which cannot be discriminated from the fault free case, i.e., no tests trigger. To increase the fault detectability, the fault $f_{i}$ is modeled as an unknown additive parameter that can be estimated in the test quantity. When the estimated fault differs too much from zero, the fault may be detected by the FDI-system. This gives the diagnosis models for the sensor faults:

$$
\begin{aligned}
F_{k}(\dot{x}, x, u) & =0 \\
y_{k, i} & =h_{k, i}(x)+f_{k} \\
\dot{f}_{k} & =0
\end{aligned}
$$

where $k=1, \ldots, 8$, and $i=1, \ldots, 8$. For the actuator faults the models are:

$$
\begin{aligned}
F_{k}\left(\dot{x}, x, u_{k-8}+f_{k}\right) & =0 \\
y_{k, i} & =h_{k, i}(x) \\
\dot{f}_{k} & =0
\end{aligned}
$$


where $k=9,10$, and $i=1, \ldots, 8$. The derivative of the faults are set equal to zero in Eqs. (9.3)-(9.4) but the dynamic of the faults can be adjusted when the CGEKFs are designed. The faults, signals, and measured quantities are summarized in Tab. 9.1 and coincide with Fig. 9.1. Since each fault is estimated correctly by one specific test quantity, thus the test quantity which estimates the fault is not sensitive to the injected fault, i.e., the fault is decoupled in the test quantity by definition.

Table 9.1: Sensor and actuator Faults.

\begin{tabular}{lccc}
\hline Signal & Quantity & Fault & Mode \\
\hline$y_{1}, y_{2}, y_{3}$ & $p_{1}, p_{3}, p_{8}$ & $f_{1}, f_{2}, f_{3}$ & $F_{p_{1}}, F_{p_{3}}, F_{p_{8}}$ \\
$y_{4}, y_{5}, y_{6}$ & $t_{2}, t_{3}, t_{7}$ & $f_{4}, f_{5}, f_{6}$ & $F_{t_{2}}, F_{t_{3}}, F_{t_{7}}$ \\
$y_{7}, y_{8}$ & $n_{C 1}, n_{T 0}$ & $f_{7}, f_{8}$ & $F_{n_{C 1}}, F_{n_{C 1}}$ \\
\hline$u_{1}, u_{2}$ & $m_{f}, P_{A}$ & $f_{9}, f_{10}$ & $F_{m_{f}}, F_{P_{A}}$ \\
\hline
\end{tabular}

\subsubsection{Constraints on Performance Deviation}

To isolate a fault, it is not enough to consider the estimated faults $f_{i}$ for the decision since more than one estimated fault $f_{i}$ can deviate from zero for a given fault mode. For the fault isolation procedure also the residuals must be considered in the decision process. As mention earlier, slow drifting bias faults can be difficult to detect in the residuals. Similar arguments are presented in Kobayashi and Simon (2005), where performed investigations show that the residuals are small even when a sensor fault is present, since the health parameters try to explain the fault by a shifting in value. Therefore, the number of health parameter (and where in the model they appear) affects the fault signature in the residuals. Thus, it is a balance between: (i) the ability to see a sensor fault in the residuals, and (ii) the ability to not see the component deterioration in the residuals. In Larsson et al. (2013), the number of health parameters were reduced to make the sensor fault isolation procedure possible when experimental data was utilized. Since no component degradation in, e.g., the compressor-turbine T1 was considered, false alarms can be generated when the compressor-turbine component deteriorates. In Kobayashi and Simon (2005), the problem is solved using an extra residual where the weighted sum of squared health parameters is compared with a threshold. Thus, using the threshold an alarm can be triggered when the deviation from the healthy base line is too large. Instead of using the weighted sum, herein a constant upper and lower limit of the health parameters are introduced which is presented in Tab. 9.2. This is a compromise between the usage of many health parameters and the fault isolation performance. Since the compressor should be washed when the performance has been reduced about $2-3 \%$ Scott (1986) a lower limit of $-4.5 \%$ is no problem. It is not common that the performance of the compressor increases, therefore a value in the range of $0.5-1.0 \%$ is reasonable. The performance of the turbine is more unsure, therefore a band interval of $\pm 2-3 \%$ is introduced for those health parameters.

Since these limits are used only for fault isolation and not for fault detection other 
Table 9.2: Health parameter upper and lower limits.

\begin{tabular}{ccc}
\hline Health Parameter & Lower Limit & Upper Limit \\
\hline$\Delta \eta_{C 1}$ & $-4.5 \%$ & $0.5 \%$ \\
$\Delta \Gamma_{C 1}$ & $-4.5 \%$ & $1.0 \%$ \\
$\Delta \eta_{T 1}$ & $-3.0 \%$ & $3.0 \%$ \\
$\Delta \Gamma_{T 1}$ & $-2.0 \%$ & $2.0 \%$ \\
\hline
\end{tabular}

types of constraints are possible to consider. At least, the upper limit for the health parameters of the compressor could be more dependent how fouled the compressor is when the fault detection alarm is triggered.

\subsubsection{Differential Algebraic Equation Form}

The fault models in Eqs. (9.3)-(9.4) are combined with the deterioration description (9.2) to get models in the form similar to Eq. (9.1). From these models $D_{i}$, the test quantities used in the FDI-system are generated. Depending of which fault that is decoupled, different health parameters are used to estimate the component deteriorations since the health parameters influence the observability and the ability to give reliable estimates when experimental data is evaluated. To decouple a fault in the sensor measuring the speed $n_{C 1}$ of the gas generator is especially difficult and gives poor estimations of the state variables if all the four health parameters are considered. When simulated data is considered, it is easier to get reliable estimation of the state variable. The focus in the work is to get reliable estimations of the state variables when experimental data is considered, therefore one health parameter is removed for the fault mode $F_{n_{C 1}}, F_{p_{3}}, F_{t_{3}}$, and $F_{t_{7}}$. These fault modes are also most difficult to isolate from each other if no limits of the health parameters are considered in the diagnosis test quantity.

Fault Free Model: The diagnosis model $D_{0}$ in the non-faulty case has the DAE form:

$$
\begin{aligned}
F_{0}(\dot{z}, z, u) & =0 \\
y & =h(z) \\
z & =\left(x, \Delta_{0}\right)^{T}
\end{aligned}
$$

where $\Delta_{0}=\left(\Delta \eta_{C 1}, \Delta \Gamma_{C 1}, \Delta \eta_{T 1}, \Delta \Gamma_{T 1}\right)^{T}$, and $x$ consists of the unknown variables.

Sensor/Actuator Fault Model: The diagnosis model $D_{k}$, in case of a fault, has the DAE form:

$$
\begin{aligned}
F_{k}(\dot{z}, z, u) & =0 \\
y & =h(z) \\
z & =\left(x, \Delta_{k}, f_{k}\right)^{T}
\end{aligned}
$$


where index $k=1,2, \ldots, 10, \Delta_{k}$ consists of the health parameters, and $x$ consists of the unknown variables. Depending of the fault model, the vector $\Delta_{k}$ has health parameter elements shown in Tab. 9.3.

Table 9.3: Health parameters utilized in diagnosis model $D_{k}$.

\begin{tabular}{c|ccccccccccc}
\hline & $\mathbf{D}_{\mathbf{0}}$ & $\mathbf{D}_{\mathbf{1}}$ & $\mathbf{D}_{\mathbf{2}}$ & $\mathbf{D}_{\mathbf{3}}$ & $\mathbf{D}_{\mathbf{4}}$ & $\mathbf{D}_{\mathbf{5}}$ & $\mathbf{D}_{\mathbf{6}}$ & $\mathbf{D}_{\mathbf{7}}$ & $\mathbf{D}_{\mathbf{8}}$ & $\mathbf{D}_{\mathbf{9}}$ & $\mathbf{D}_{\mathbf{1 0}}$ \\
\hline & $F_{N F}$ & $F_{p_{1}}$ & $F_{p_{3}}$ & $F_{p_{8}}$ & $F_{t_{2}}$ & $F_{t_{3}}$ & $F_{t_{7}}$ & $F_{n_{C 1}}$ & $F_{n_{T 0}}$ & $F_{m_{f}}$ & $F_{P_{A}}$ \\
\hline$\Delta \eta_{C 1}$ & $\mathrm{x}$ & $\mathrm{x}$ & $\mathrm{x}$ & $\mathrm{x}$ & $\mathrm{x}$ & $\mathrm{x}$ & $\mathrm{x}$ & $\mathrm{x}$ & $\mathrm{x}$ & $\mathrm{x}$ & $\mathrm{x}$ \\
$\Delta \Gamma_{C 1}$ & $\mathrm{x}$ & $\mathrm{x}$ & $\mathrm{x}$ & $\mathrm{x}$ & $\mathrm{x}$ & $\mathrm{x}$ & $\mathrm{x}$ & & $\mathrm{x}$ & $\mathrm{x}$ & $\mathrm{x}$ \\
\hline$\Delta \eta_{T 1}$ & $\mathrm{x}$ & $\mathrm{x}$ & & $\mathrm{x}$ & $\mathrm{x}$ & & & $\mathrm{x}$ & $\mathrm{x}$ & $\mathrm{x}$ & $\mathrm{x}$ \\
$\Delta \Gamma_{T 1}$ & $\mathrm{x}$ & $\mathrm{x}$ & $\mathrm{x}$ & $\mathrm{x}$ & $\mathrm{x}$ & $\mathrm{x}$ & $\mathrm{x}$ & $\mathrm{x}$ & $\mathrm{x}$ & $\mathrm{x}$ & $\mathrm{x}$ \\
\hline
\end{tabular}

\subsubsection{State Space Form}

The purpose of the diagnosis model is to introduce faults and health parameters in an easy manner. Since the diagnosis model is equation based, where algebraic and dynamic constraints are mixed, some equation transformations are necessary to perform to get it into a state space form. The first step is to transform the set of equations in (9.5)-(9.6) automatically to a form which can be used in a diagnosis test quantity based on state space observers. To get the test equation, two main steps are performed: (i) check and reduce the DAE-index of the system where Pantelides algorithm (Pantelides, 1988) is utilized, and (ii) find the over-determined part and remove the exactly determined part using the Dulmage-Mendelsohn decomposition (Dulmage and Mendelsohn, 1958). For these two steps, the structural model (Blanke et al., 2003) of the diagnosis model is used. For a more detailed overview of each step in the design procedure, see Larsson et al. (2014a) since these transformation steps are not trivial. After the transformations, the test equations of diagnosis model $D_{k}$ in a state space form are:

$$
\begin{aligned}
\dot{x}_{1} & =f_{k}\left(x_{1}, u\right) \\
y & =h_{k}\left(x_{1}\right)
\end{aligned}
$$

where $x_{1}$ represents the dynamic variables, $u$ represents the known input signals, and $y$ represents the known measurements. The functions $f_{k}$ and $h_{k}$ have appropriate dimension. The index $k$ is the same as used in (9.5)-(9.6). In the vector $x_{1}$, the health parameters and the faults are included.

\subsection{Test Quantity Design}

The main objective in this section is to present how the test quantities, used in the FDIsystem, are designed. Each test quantity $T_{i}$ consists of a nonlinear Kalman filter which is generated from the diagnosis models $D_{i}$ in Eqs. (9.5)-(9.6). The outputs from the test 
quantities, except for the non-faulty case, are: (i) a logical value $L_{i}$ which represents if the test has triggered or not, and (ii) a logical value $L_{i}^{f}$ which represents if a fault is detected or not, and (iii) an estimation $\hat{f}_{i}$ of the fault $f_{i}$. When none of the considered fault modes are present in the system the fault estimates $\hat{f}_{i}$ should be small, and $L_{i}, L_{i}^{f}$ should not triggered. Thus, when the fault estimate in any of the tests is large, a fault is present in the system and a fault detection alarm should be triggered. To detect the fault, the fault signal $\hat{f}_{i}$ are filtered using the CUSUM algorithm (Page, 1954; Gustafsson, 200o) and compared with a static threshold. When any of the fault estimates exceed its threshold, a fault detection alarm is triggered.

To determine if the test quantity $T_{i}$ should alarm or not, the output residuals $r_{i}^{j}$ from the filters are first filtered using the CUSUM algorithm. Then a logical OR operation is applied to the CUSUM tests $T_{i}^{j}$ to decide if the test quantity is triggered or not. This means that if any of the CUSUM tests $T_{i}^{j}$ are triggered, the test quantity $T_{i}$ will also trigger an alarm. The test quantity $T_{i}$ is then used in the fault isolation procedure. A schematic view of the test quantity is shown in Fig. 9.2.

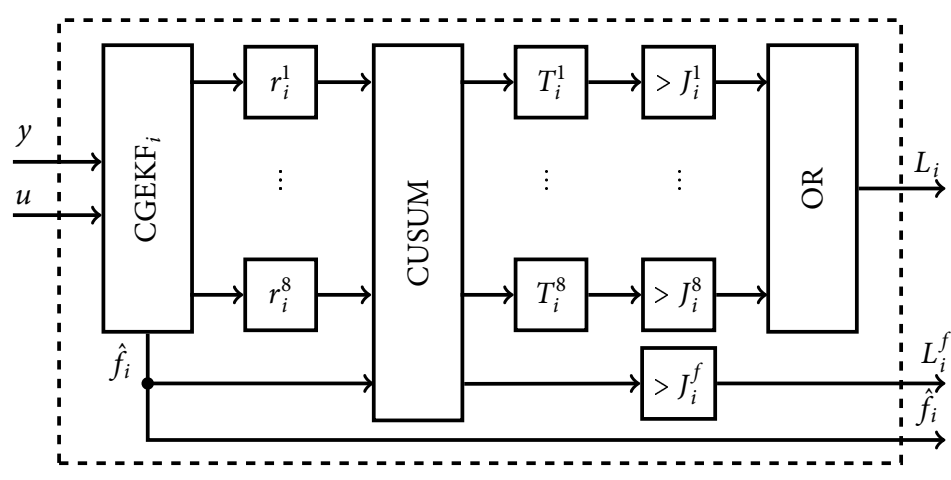

Figure 9.2: Test quantity $T_{i}$ of diagnosis model $D_{i}$.

\subsubsection{Constant Gain Extended Kalman Filter}

To estimate state variables and component deterioration in the gas turbine, a common approach in the gas turbine diagnosis literature is to use filters. The filters are often Kalman based and are linear in Luppold et al. (1989); Litt (2007); Simon and Armstrong (2013); Simani and Patton (2008) and nonlinear in Kobayashi et al. (2005); Naderi et al. (2011); Dewallef et al. (2006). A special type of nonlinear Kalman filters is the constant gain extended Kalman filter (CGEKF) (Safonov and Athans, 1978) which was investigated in a gas turbine application in papers Kobayashi et al. (2005); Sugiyama (2000) with success. Here, the test quantity of each diagnosis model $D_{i}$ is written in the form:

$$
\begin{aligned}
& \dot{\hat{x}}_{i}=f_{i}\left(\hat{x}_{i}, u\right)+K_{i}\left(y-\hat{y}_{i}\right) \\
& \hat{y}_{i}=h_{i}\left(\hat{x}_{i}\right)
\end{aligned}
$$


where the functions $f_{i}$ and $h_{i}$ can be nonlinear, $u$ represents the input signals, $y$ represents the measurement signals, and $K_{i}$ is the feedback gain which can be considered as a design parameter. For the CGEKF the constant matrix $K_{i}$ is calculated in advance for a given stationary point of (9.7) using the noise covariance matrices $Q_{i}$ and $R_{i}$. The covariance matrix $R_{i}$ is diagonal with elements belonging to the standard deviation of measurement noise for each sensor which is presented in Tab. 9.4. The covariance matrix $Q_{i}$ is also diagonal where the elements representing the model uncertainty of each state variable. Especially the states of the health parameters have been given small uncertainty variances, i.e., the feedback term for these states have a small impact on the estimations.

Table 9.4: Standard deviation $\sigma$ of the measurement noise.

\begin{tabular}{lcl}
\hline Signal & Quantity & $\sigma$ \\
\hline$y_{1}, y_{2}, y_{3}$ & $p_{1}, p_{3}, p_{8}$ & $0.5 \%$ \\
$y_{4}, y_{5}, y_{6}$ & $t_{2}, t_{3}, t_{7}$ & $0.5 \%$ \\
$y_{7}, y_{8}$ & $n_{C 1}, n_{T 0}$ & $0.2 \%$ \\
\hline$u_{1}, u_{2}$ & $m_{f}, P_{A}$ & $0.2 \%$ \\
\hline
\end{tabular}

\subsubsection{CUSUM Filtering}

For change detection in signals, a suitable choice is to use the CUSUM algorithm (Page, 1954; Gustafsson, 2000). The CUSUM algorithm, together with a static threshold $J_{i}^{j}$ are used to decide if the residual $r_{i}^{j}$ and fault estimate $\hat{f}_{i}$ indicates an abnormal behavior according to the sensor or actuator faults. The CUSUM test quantity $T_{i}^{j}$ for each residual is computed

$$
T_{i}^{j}(t)=\max \left(0, T_{i}^{j}(t-1)+\left|r_{i}^{j}(t)\right|-v_{i}^{j}\right), \quad T_{i}^{j}(0)=0
$$

where $t$ is time, $i \in[0, \ldots, 10], j \in[1, \ldots, 8], v_{i}^{j}$ is a tuning parameter to ensure that $T_{i}^{j}(t)<0$ in the fault free case, and $\left|r_{i}^{j}(t)\right|$ is the absolute value of residual in Eq. (9.10). The threshold $J_{i}^{j}=J$ is more or less equal for all CUSUM tests since the normalization of the residuals in (9.10) was performed. The test quantity triggers an alarm when $T_{i}^{j}(t)$ exceeds the specified threshold $J$. The design parameter $v_{i}^{j}$ is tuned so the test does not give any unnecessary alarms in the fault free case, i.e., no false alarms. A good rule of thumb is that $v_{i}^{j}$ has the same order of magnitude as the residual $r_{i}^{j}$ in the fault free case, i.e., here the parameter is approximately 1 . The CUSUM filtering of the fault estimate $f_{i}$ is analogue with test quantity $T_{i}^{j}$ filtering procedure.

In an ideal case, when a slow varying fault $f_{i}$ is present in the sensor or actuator signals all test quantities except $T_{i}$ should trigger. In practice, this is not always true since, e.g., measurement noise, fault size, and model uncertainty are always present together with the tuning procedure of the CGEKFs and the CUSUM tests. All these things affect the detectability and reaction rate of each test quantity. 


\section{Residuals}

The output from each Kalman filter is an estimation of the state variables $\hat{x}_{i}$ and the sensor values $\hat{y}_{i}$. The estimated sensor values from each filter are used to construct residuals $r_{i}^{j}$ in the form:

$$
r_{i}^{j}=\left(\hat{y}_{i}^{j}-y_{i}^{j}\right) / \sigma_{i}^{j}
$$

where $i \in[1, \ldots, 10], j \in[1, \ldots, 8]$, and $\sigma_{i}^{j}$ is the standard deviation of $r_{i}^{j}$ in the fault free case, i.e., the same noise as presented in Tab. 9.4. Since the measurement sequence is available offline, the residuals can be normalized with the standard deviation of the residuals in the fault free case. With this normalization, the signals are in the same interval.

\section{Faults}

The outputs from the CGEKFs are, e.g., estimations of the faults $f_{1}, \ldots, f_{10}$. When no faults of the considered fault modes are present in the system, the fault estimates should be low. When any of the fault estimates are large, the system is no longer non-faulty and a fault detection alarm should be triggered. To determine if any of the fault estimates are large, the CUSUM algorithm is used together with a static threshold. Since a specific fault can affect fault estimates in many tests, the fault detection signal can only be used for fault detection and not for isolation.

\subsection{Fault Isolation Method}

The main objective in this section is to present how the isolation procedure of single sensor and actuator faults is performed using the developed FDI-system shown in Fig. 9.3. The inputs to the FDI-system are known quantities such as sensor and actuator signals. The outputs are the estimated states $\hat{x}$, a suggestion if the compressor should be washed $W$, and a set of diagnoses $D$ which are consistent with the observations. The FDI-system is based on hypothesis testing and there is one hypothesis for each sensor and actuator failure. Such an approach is standard in general diagnosis methodology (Blanke et al., 2003) and similar approach was also utilized in Larsson et al. (2010); Kobayashi and Simon (2005). Thus, let $H_{i}, i=1, \ldots, 10$ denotes the hypothesis that the sensor $y_{i}$ or actuator $u_{j}$ is faulty and let $H^{0}$ denote the null-hypothesis that no sensor or actuator is faulty. For each fault hypothesis $H_{i}$ the test quantity $T_{i}$ is designed given the diagnosis model $D_{i}$ where an additional state variable $f_{i}$ is introduced in the measurement sensor $y_{i}$ or in the actuator signal $u_{j}$. When hypothesis $H_{i}$ is true, all filters except the filter in $T_{i}$ cannot estimate the faulty sensor or actuator value correctly and the filters have ideally a large error in the residuals. When hypothesis $H_{i}$ is true, the test $T_{i}$ is used to estimate the correct state variables which can be used for supervision and control purposes. Since the fault is already estimated, this estimate is used to compensate for the faulty input signal to the test $T_{0}$. Thus, when a fault is diagnosed it is assumed that the fault is decoupled in the test quantity $T_{0}$.

To reject the null-hypothesis $H^{0}$, some of the fault estimates $\hat{f}_{1}, \ldots, \hat{f}_{10}$ should indicate a major deviation from zero. When the null-hypothesis is rejected, the system can 


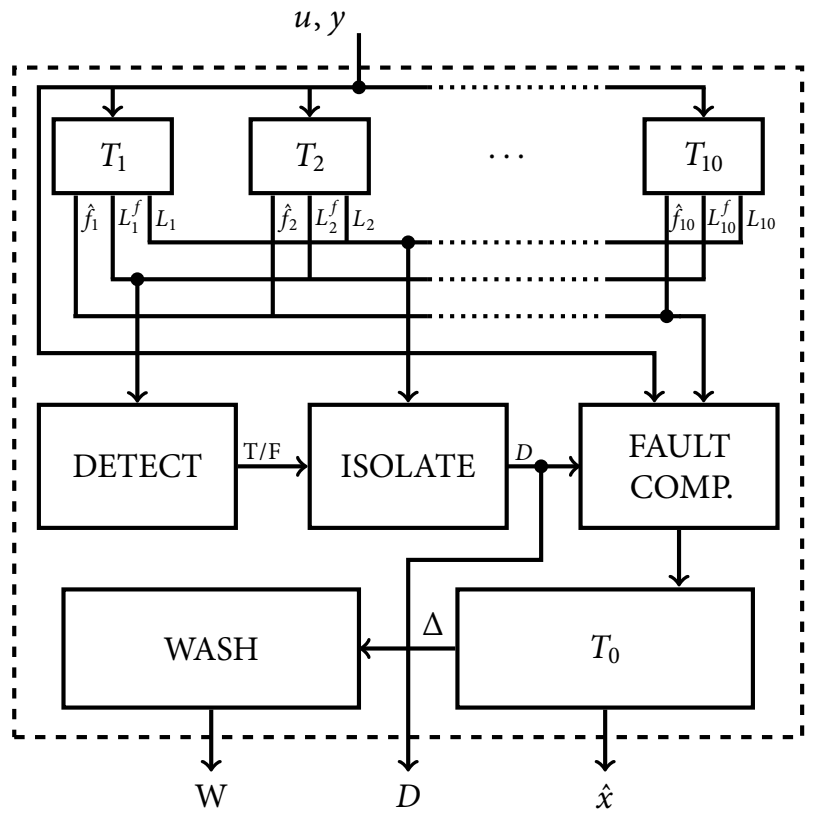

Figure 9.3: FDI-system.

no longer be non-faulty. If the hypothesis $H^{0}$ is not rejected, the system is assumed to be non-faulty and the input signals to the test quantity $T_{0}$ are not modified and the test can directly be used to estimate the performance.

The Kalman filters are used to estimate the outputs $\hat{y}$ and combine these outputs with the real measurement signals $y$ to get the residuals. The residuals are then filtered using CUSUM and compared with a given threshold value $J_{i}^{j}$, which results in a logical value $L_{i}$. The logical values $L_{i}$ are then used in the fault isolation logical component ISOLATE (Fig. 9.3) where the diagnoses consistent with the hypotheses are determined. In the component FAULT COMP., the measurement and actuator signals are compensated for the fault in the signals when a unique diagnosis $D$ is stated. The test $T_{0}$ is then used to estimate the state variables which can be used to, e.g., supervise the turbine inlet temperature TIT and the degree of compressor fouling. The component WASH determines if the deterioration level of compressor performance is sufficient to perform a compressor wash. After the wash, the health parameters for the compressor are reset. In the component DETECT, it is determined if the $H^{0}$ hypothesis should be rejected or not. When the $H^{0}$ hypothesis is not rejected, the ISOLATE component is not active since it is assumed that the system is non-faulty. 


\subsubsection{No Fault Hypothesis $H^{0}$}

When the gas turbine system is non-faulty, no tests in the FDI-system should trigger. To reduce the number of unnecessary false alarms, and increase the fault detectability the fault estimates: $\hat{f}_{1}, \ldots, \hat{f}_{10}$ are used to determine if the $H^{0}$ hypothesis should be rejected or not. To trigger a fault detection alarm it is enough if any of the CUSUM tests of $\hat{f}_{i}$ exceeds its threshold. If hypothesis $H^{0}$ is not rejected, it is assumed that the system is non-faulty.

\subsubsection{Fault Hypothesis $H_{i}$}

When one or more of the CUSUM tests of the fault estimate $\hat{f}_{i}$ trigger, the $H^{0}$ hypothesis is no longer valid. For each test $T_{i}$ that has alarmed, the fault hypothesis $H_{i}$ can be removed from the possible set of valid hypotheses. When only one test $T_{j}$ is left in the not alarmed test set the only valid hypothesis is $H_{j}$. This results in the single sensor or actuator diagnosis $D_{j}$. When a single diagnosis $D_{j}$ is obtained, the estimated fault $f_{j}$ is used to compensate for the faulty signal in the test quantity $T_{0}$. Depending on the actual diagnosis, and the current component deterioration, the accuracy of the estimated fault varies since different numbers of health parameters are used in the test quantities $T_{1}, \ldots, T_{10}$. For example, mass flow degradation in the compressor $\mathrm{C} 1 \mathrm{can}$ affect the estimation of fault $f_{7}$ (which will be shown in Fig. 9.10). To compensate for this phenomenon, the detection threshold for fault $f_{7}$ should be adjusted little higher.

Since all input and output signals are used in each test quantity, the test is in theory sensitive to all faults which mean that ideally a fault cannot be decoupled and an isolation procedure is not possible. It takes some time for the fault parameter $\hat{f}_{i}$ to estimate the fault $f_{i}$, and when the fault has reached the correct value it is assumed that the fault is decoupled in the test quantity $T_{i}$ and is not sensitive to the fault $f_{i}$. Since it takes some time for the fault estimate to reach the fault level, a correct diagnosis cannot be guaranteed during this period of time.

\subsubsection{Component Failure and Foreign Object Damage}

For the case when all tests $T_{i}$ alarm, no hypothesis is no longer valid since a single fault assumption is considered. A probably scenario is that a sudden fault with large amplitude has occurred and it takes time for the fault estimate to reach the fault level. Another explanation is that a rapid change in component performance occurs, i.e., a component failure (CF) or some kind of foreign object damage (FOD). Since upper and lower limits (Tab. 9.2) of the health parameters are introduced, a large shift in the performance parameters is visible in the residuals. The CF and FOD symptoms can only be detected since no fault models for these kinds of symptoms are considered, thus it is not possible to isolate these fault events. In Tab. 9.5, the test alarm scheme is summarized. 
Table 9.5: FDI scheme.

\begin{tabular}{cccc}
\hline $\begin{array}{c}\text { Fault } \\
\text { Detected }\end{array}$ & $\begin{array}{c}\text { Number of } \\
\text { Alarms } \mathbf{L}_{\mathbf{i}}\end{array}$ & Description & $\begin{array}{c}\text { Fault } \\
\text { Estimated }\end{array}$ \\
\hline 0 & $0 \leq n \leq 9$ & $\mathrm{NF}, H^{0}$ valid & 0 \\
1 & $1 \leq n \leq 8$ & Multiple $H_{i}:$ s valid & 0 \\
1 & $n=9$ & Unique $H_{i}$ valid & 1 \\
$0 / 1$ & $n=10$ & $\mathrm{CF}, \mathrm{FOD}, \mathrm{MF}$ & 0 \\
\hline
\end{tabular}

\subsection{Simulation Results}

The main objective in this section is to investigate how the proposed FDI-system responds to the various fault scenarios when: (i) performance deterioration is introduced in the components, (ii) the speed and power of the power turbine are varied, and (iii) noise in the measurement signals is introduced. Since data from the simulation platform presented in Larsson et al. (2014a) is considered, the case study can be performed under controlled forms and the correct values of the parameters are also known for comparison. The secondary objective with the case study is to present and compare how the introduced limits of health parameters influence the reaction ability of the tests.

\subsubsection{Simulation Setup}

The input signals to the simulation platform are: (i) demand power, (ii) frequency of the driven component, (iii) ambient condition such as pressure $p_{0}$, temperature $t_{0}$, and relative humidity $\varphi_{0}$ of the ambient air. In the following simulation plots, only the demand power and frequency of the driven component are varied. The other inputs are kept constant for simplicity. Further studies, which have been performed and not shown here, indicate that it is possible to also varying the ambient conditions with similar results. The change in frequency of the driven component is a direct function of the speed of the power turbine. Therefore, the speed is shown here and not the frequency. In the simulation platform, it is also possible to introduce slow performance deterioration in components. Here, deteriorations in compressor $\mathrm{Cl}$, and turbine $\mathrm{T} 1$ are considered. The injected performance deterioration is presented in Tab. 9.6 were also the slope of the deterioration is shown. Given the simulation setup, the controller calculates the opening angles for the valves in the fuel system. Given the angles, the mass flow of fuel is calculated by the model and the mass flow is considered to be an input signal to the FDI-system. After the data is collected, Gaussian noise with zero mean and standard deviation according to Tab. 9.4 is added to the signals. The simulated data which is used as inputs to the FDI-system is shown in Fig. 9.4. A higher demand power increases the fuel consumption $u_{1}$, the compressor discharge pressure $p_{3}$ and temperature $t_{3}$, exhaust temperature $t_{7}$, and speed of the gas generator $n_{C 1}$. 


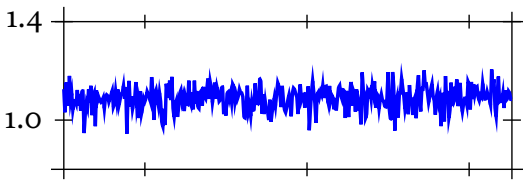

(a) $y_{1}[$ bar $]$

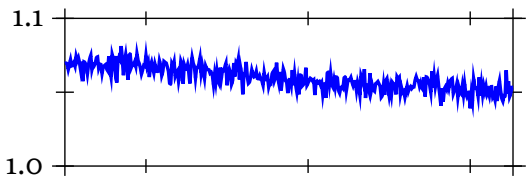

(c) $y_{3}[$ bar $]$

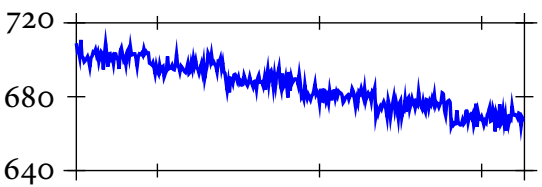

(e) $y_{5}[\mathrm{~K}]$

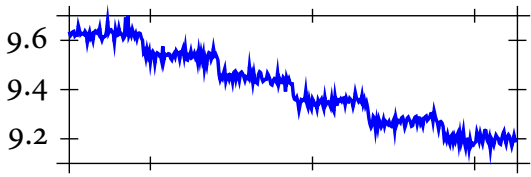

(g) $y_{7}[\mathrm{krpm}]$

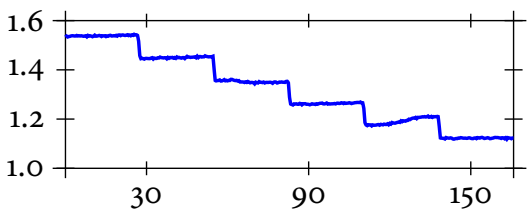

(i) $u_{1}[\mathrm{~kg} / \mathrm{s}]$

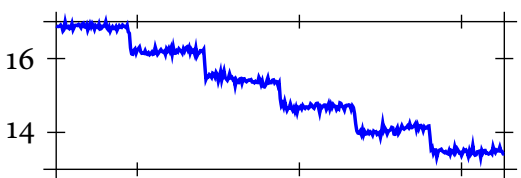

(b) $y_{2}[\mathrm{bar}]$

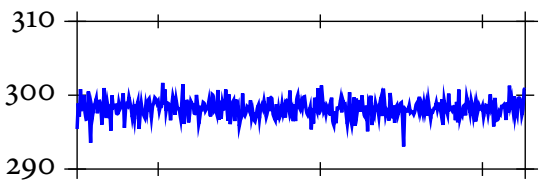

(d) $y_{4}[\mathrm{~K}]$

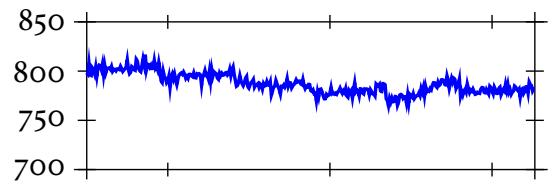

(f) $y_{6}[\mathrm{~K}]$

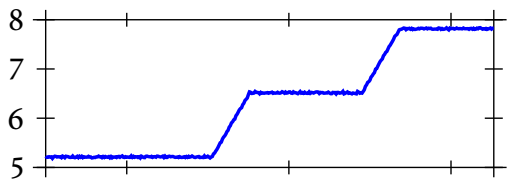

(h) $y_{8}[\mathrm{krpm}]$

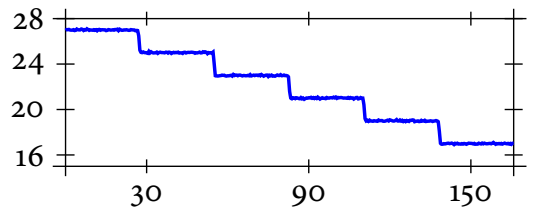

(j) $u_{2}[\mathrm{MW}]$

Figure 9.4: Simulated measurement signals corresponding to a variation in applied power simultaneously the performance in $\mathrm{Cl}$ and $\mathrm{T} 1$ deteriorates. The measurements are gathered using the simulation platform where Gaussian noises are added to the signals according to Tab. 9.4. 
Table 9.6: Performance Deteriorations and Control input Signals.

\begin{tabular}{c|cc}
\hline $\begin{array}{c}\text { Performance } \\
\text { Parameter }\end{array}$ & $\begin{array}{c}\text { Injected } \\
\text { Deterioration [\%] }\end{array}$ & $\begin{array}{c}\text { Duration } \\
\text { [days] }\end{array}$ \\
\hline$\eta_{C 1}$ & 3.0 & 130 \\
$\Gamma_{C 1}$ & 2.5 & 130 \\
$\eta_{T 1}$ & 1.0 & 130 \\
$\Gamma_{T 1}$ & 1.0 & 130 \\
\hline Demand Power & Step [MW] & Duration [days] \\
\hline$P_{A}$ & -2 & 27 \\
\hline Demand Speed & Step [rpm] & Duration [days] \\
\hline$n_{T 0}$ & $\approx 1500$ & $\approx 50$ \\
\hline
\end{tabular}

\subsubsection{Fault Modes}

For the given scenario of input signals and component deteriorations, faults with amplitudes given in Tab. 9.7 are investigated in the simulation study. Two different kind of bias

Table 9.7: Fault modes and amplitude of injected sensor or actuator faults.

\begin{tabular}{l|ccc}
\hline Fault Mode & Signal & Quantity & Amplitude \\
\hline$F_{N F}$ & - & - & - \\
$F_{p_{1}}, F_{p_{8}}$ & $y_{1}, y_{3}$ & $p_{2}, p_{8}$ & $\pm 4 \%$ \\
$F_{p_{3}}$ & $y_{2}$ & $p_{3}$ & $-4 \%,+5 \%$ \\
$F_{t_{2}}$ & $y_{4}$ & $t_{2}$ & $\pm 3 \%$ \\
$F_{t_{3}}$ & $y_{5}$ & $t_{3}$ & $-4 \%,+3 \%$ \\
$F_{t_{7}}$ & $y_{6}$ & $t_{7}$ & $-3 \%,+5 \%$ \\
$F_{n_{C 1}}, F_{n_{T 0}}$ & $y_{7}, y_{8}$ & $n_{C 1}, n_{T 0}$ & $\pm 5 \%$ \\
$F_{m_{f}}, F_{P_{A}}$ & $u_{1}, u_{2}$ & $m_{f}, P_{A}$ & $-5 \%,+10 \%$ \\
\hline
\end{tabular}

fault signatures are investigated which propagate: (i) abrupt (step fault), and (ii) slowly drifting bias (ramp fault) to a given fault amplitude. The fault amplitudes in the table are chosen in a way so the faults can be detected and isolated by the FDI-system. For some faults, the amplitude is not symmetric, and it also depends where in the sequence the fault is injected since also component deteriorations are present in the data. The faults in the input signals are large because they are difficult to isolate from each other, but it is possible to isolate those faults from the other faulty modes even with smaller amplitude.

\subsubsection{Fault Free Mode: $F_{N F}$}

In the fault free case, the FDI-system uses the input-, and output signals shown in Fig. 9.4. No false alarms are generated by the FDI-system, and the estimated performance 
deterioration (blue line) is shown in Fig. 9.5 together with the injected deterioration (red line). As the figure indicates, it is no problem to estimate the component deteriorations in the fault free mode although the applied power and speed of the power turbine are varied.

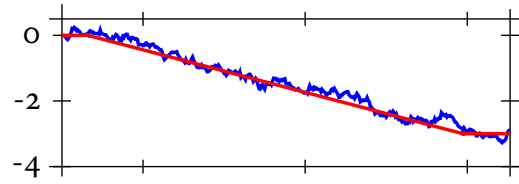

(a) $T_{0}: \Delta \eta_{C 1}[\%]$

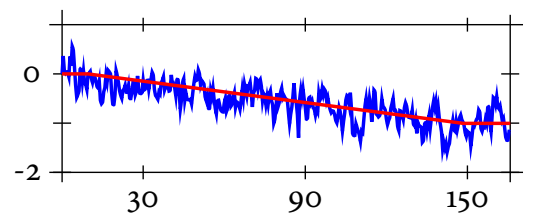

(c) $T_{0}: \Delta \eta_{T 1}[\%]$

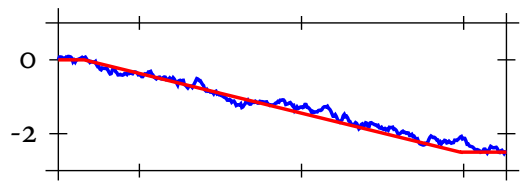

(b) $T_{0}: \Delta \Gamma_{C 1}[\%]$

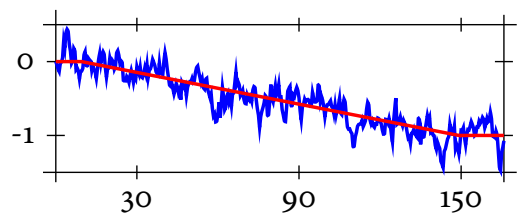

(d) $T_{0}: \Delta \Gamma_{T 1}[\%]$

Figure 9.5: Estimated (blue line) and injected (red line) performance deterioration corresponding to the measurement signals in Fig. 9.4. The estimation is performed using the test quantity $T_{0}$ in the FDI-system together with a data set where no fault is injected.

\subsubsection{Sensor Fault Modes: $F_{p_{3}}, F_{t_{3}}$, and $F_{t_{7}}$}

To detect fault in sensors measuring: (i) pressure $p_{3}$, (ii) temperature $t_{3}$, and (iii) temperature $t_{7}$ can be little tricky since the health parameters compensate, in some sense, for the faulty sensor value. The investigated step fault can be seen in the residuals during a short period of time, but when the fault has decline it is difficult to discriminate the faulty sequence from the non-faulty measurements since noise is present. A slow varying fault is more difficult to isolate since the fault behaves similar to component deteriorations and therefore are the tests not triggered. To reduce the problem, an upper and lower limit of the health parameters is introduced according to Tab. 9.2. The purpose with the limitations is to prevent the health parameters to compensate for the fault and hence have residuals that can be discriminated from the non-faulty case. In Fig. 9.6-9.7, a step fault with an amplitude of -4 , and $+3 \%$ is injected in the temperature sensor $y_{5}$ measuring $t_{3}$ and the results are shown for the test quantity $T_{2}$ which decouple a fault in the sensor measuring pressure $p_{3}$. Since $T_{2}$ estimates a fault in $p_{3}$, this test quantity should trigger an alarm. When the $+3 \%$ fault is injected in the sensor (blue line), the $\Delta \Gamma_{T 1}$ parameter reacts drastically and after some time the residuals go back to zero as shown in Fig. 9.7. For better isolation performance, an upper and lower limit of the health parameters are introduced for the two other fault scenarios which gives a more distinct fault signature (red and green line) in the residuals. The limits of the health parameters does not affect the signal used for detection, since the correct hypothesis test 


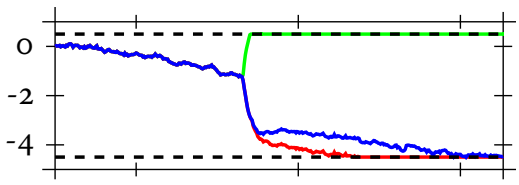

(a) $T_{2}: \Delta \eta_{C 1}[\%]$

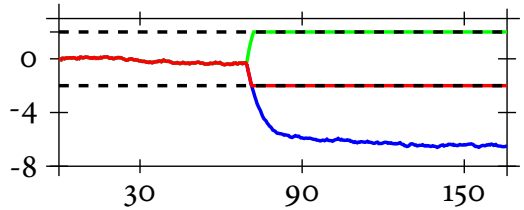

(c) $T_{2}: \Delta \Gamma_{T 1}[\%]$

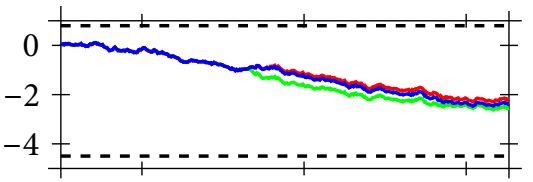

(b) $T_{2}: \Delta \Gamma_{C 1}[\%]$

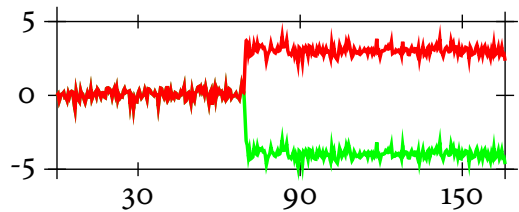

(d) $T_{5}: \hat{f}_{5}[\%]$

Figure 9.6: Health parameter estimates corresponding to three different fault cases where a step fault is injected (at day 70) in the sensor $y_{5}$ which measure the compressor discharge temperature $T_{3}$. The estimations are performed using a fault with an amplitude of $+3 \%$ (red line), with an amplitude of $-4 \%$ (green line), and with an amplitude of $+3 \%$ (blue line). For the two first fault cases, a maximum and minimum limit of the deterioration level is introduced to maintain higher fault isolability using the residuals. The limits are not affecting the fault detectability since test $T_{5}$ estimates the fault correctly (d).

$T_{5}$ estimates the injected fault correctly as shown in in Fig. 9.6d. The goal with the health parameters is to decouple component deteriorations, and when the health parameter hits the limits this ability can disappear which Fig. 9.7b indicates. In the figure, it is clear that the component deterioration affects the residuals when the health parameters had hit theirs limits. But here, only one residual in the test needs to exceed its threshold to trigger an alarm. Thus, it is sufficient that residual $r_{8}$ reacts clearly.

\subsubsection{Actuator Fault Modes: $F_{m_{f}}$ and $F_{P_{A}}$}

Two actuator faults (mass flow of fuel $m_{f}$ and generated power $P_{A}$ ) are considered in the simulation study. The investigation shows that faults in the input signals are difficult to isolate from each other, but it is easier to isolate these faults from faults in the output signals. However, to isolate faults in the input signals from each other require larger fault amplitudes, since tests $T_{9}$, and $T_{10}$ are not triggered for respective fault. The residuals which give best reactions, for each fault with a positive and negative amplitude, are shown in Fig. 9.8.

To summarize the test reaction, all test quantities are viewed in Fig. 9.9 where a step fault with an amplitude of $+5 \%$ in the input signal $u_{1}$ is diagnosed. All tests alarm just after the fault is introduced, but after a short period of time the test $T_{9}$ stops trigger, which results in the only valid hypothesis $H_{9}$. At day 150, the component deterioration has compensated for the fault in test $T_{10}$ and the test is not alarming anymore. Therefore, the fault size is increased to $+10 \%$ to get distinct diagnoses for the whole sequence. 


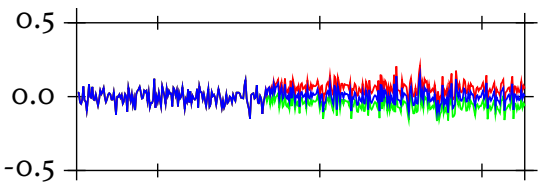

(a) $T_{2}: r_{2}[$ bar $]$

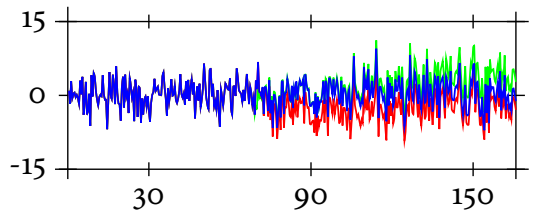

(c) $T_{2}: r_{6}[\mathrm{~K}]$

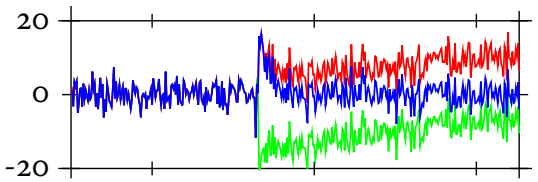

(b) $T_{2}: r_{5}[\mathrm{~K}]$

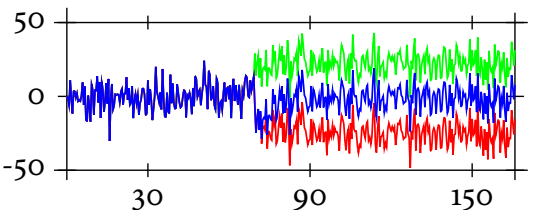

(d) $T_{2}: r_{8}[\mathrm{~K}]$

Figure 9.7: A subset of the residuals corresponding to the health parameter estimation in Fig. 9.6. With the introduced limits of the health parameters, the separation from the fault free case is more distinct.

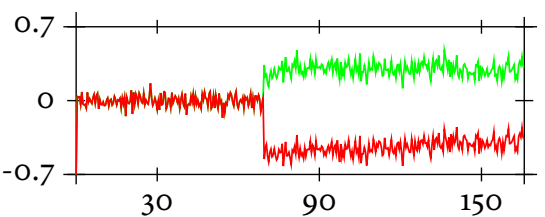

(a) $T_{10}: r_{2}[$ bar $]$

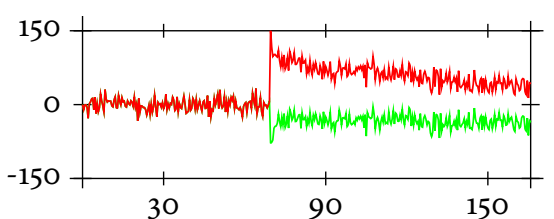

(b) $T_{9}: r_{8}[\mathrm{rpm}]$

Figure 9.8: Two residuals corresponding to a fault in $u_{1}$ (a), and in $u_{2}$ (b). The amplitudes of the faults are $-5 \%$ (green line), and $+10 \%$ (red line). The residuals are performed using the test quantity $T_{10}$ in (a), and test quantity $T_{9}$ in (b).

\subsubsection{Sensor Fault Mode: $F_{n_{C 1}}$}

In the final test case, a fault in the sensor measuring speed $n_{C 1}$ of the gas generator is investigated. This fault case is interesting since the test $T_{7}$ does not compensate for deterioration in mass flow of the compressor. When the health parameter $\Delta \Gamma_{C 1}$ is considered in the model, it is difficult to get state variable estimates which not diverge and the parameter is therefore omitted in the model. It is no problem to detect and isolate a fault with an amplitude $\pm 5 \%$, and all tests except $T_{7}$ are triggered.

The estimated fault $\hat{f}_{7}$ is used to compensate for the fault in the inputs to the $T_{0}$ test. The result of the estimations is shown in Fig. 9.10, where all health parameters except $\Delta \eta_{C 1}$ follow, more or less, the injected degradation trajectory. As expected, the estimation of $\Delta \Gamma_{C 1}$ in Fig. 9.10b is zero since the test $T_{7}$ does not estimate the degradation. Instead, the deterioration is captured by the fault parameters which Fig. 9.10e-f indicates. The impact of the degradation in the fault estimate is much smaller than the injected fault which not triggers any alarms, i.e., false alarms. 


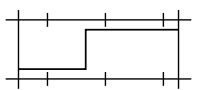

(a) $T_{1}$

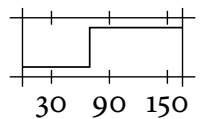

(f) $T_{6}$

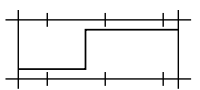

(b) $T_{2}$

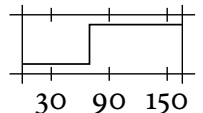

(g) $T_{7}$

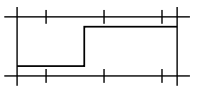

(c) $T_{3}$

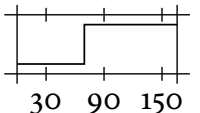

(h) $T_{8}$

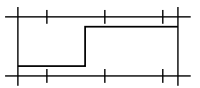

(d) $T_{4}$

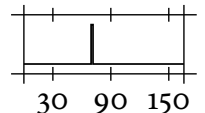

(i) $T_{9}$

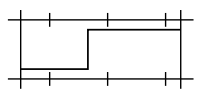

(e) $T_{5}$

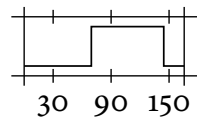

(j) $T_{10}$

Figure 9.9: Logical decision of the test quantities $T_{1}, \ldots, T_{10}$ when a fault with amplitude $+5 \%$ in the input signal $u_{1}$ is diagnosed. The logical test $T_{10}$ is not triggered after day 150 since the residual goes towards zero similar to the results in Fig. 9.8a.

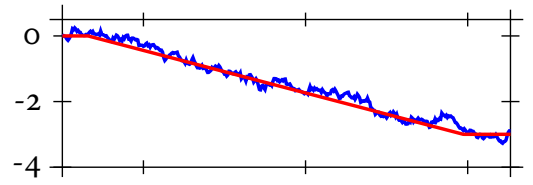

(a) $T_{0}: \Delta \eta_{C 1}[\%]$

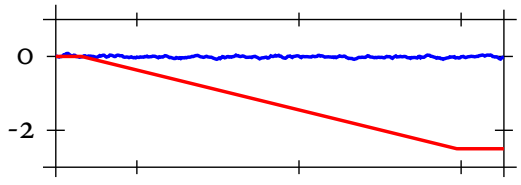

(b) $T_{0}: \Delta \Gamma_{C 1}[\%]$

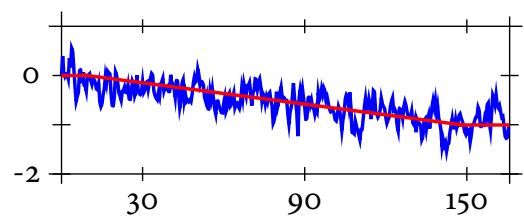

(c) $T_{0}: \Delta \eta_{T 1}[\%]$

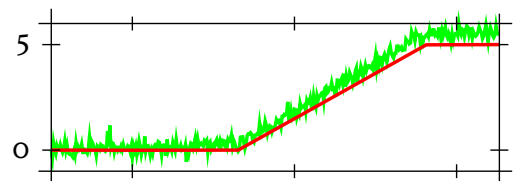

(e) $T_{7}: \hat{f}_{7}[\%]$

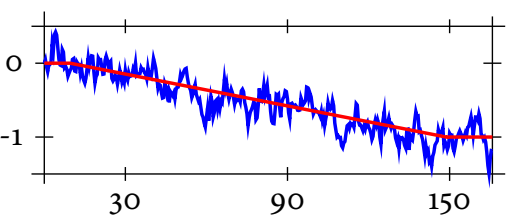

(d) $T_{0}: \Delta \Gamma_{T 1}[\%]$

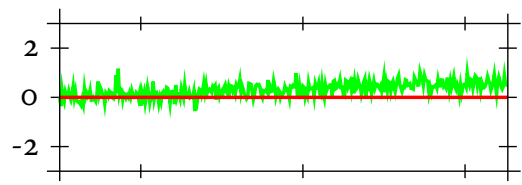

(f) $T_{7}: f_{7}-\hat{f}_{7}[\%]$

Figure 9.10: Health estimation by test $T_{0}$ (blue line), and injected performance deterioration (red line) corresponding to a fault case with a fault in the sensor measuring the speed $n_{C 1}$ of the gas generator. All health parameters except $\Delta \Gamma_{C 1}$ follow the injected component deterioration. In (e), the estimated (green) and injected (red) fault is shown. In (f), the difference between the estimated and injected fault is shown and represents, in some sense, the injected mass flow deterioration. 


\subsubsection{Summary}

The simulation study shows that all (step and ramp) faults presented in Tab. 9.7 are detectable with the developed FDI-system simultaneously as the component deteriorations are supervised. All faults are isolable from each other except faults in: (i) the sensor measuring $n_{T 0}$, and (ii) the input signal $P_{A}$. Thus, it is not possible to discriminate between a fault in the speed of the power turbine and the demand power for the simulated data. Faults in the sensors measuring: (i) discharge pressure $p_{3}$, (ii) discharge temperature $t_{3}$, and (iii) exhaust temperature $t_{7}$ are more difficult to isolate than the other faults since the fault signature may disappear in the residuals. To reduce the problem, an upper and a lower limit of the health parameters are introduced. The disadvantages with using an upper and a lower limit of the health parameters are: (i) it can be difficult to find/use suitable values of the limits, and (ii) when the health parameter has reach the limit the component deterioration is no longer decoupled in the test. For the second disadvantage, this can be a problem only for the diagnosis procedure since the correct fault hypothesis estimates the injected fault correctly.

\subsection{Experimental Case Studies}

In this section, the design of the constructed fault tolerant FDI-system is evaluated using experimental data from the gas turbine mechanical drive site. The objectives of the evaluation are to: (i) detect and isolate faults in sensor and actuator signals, (ii) supervise the health state of the engine, and (iii) use the FDI-system to suggest when it is a good idea to, e.g., wash the compressor due to fouling. The main focus with the investigation of the FDI-system is to evaluate how well the fault tolerant supervision system performs in the presence of a single sensor or actuator fault. This question is important since it is difficult, for some specific faults, to discriminate between a fault and degradation in performance especially when the fault propagation is slow. At the gas turbine site, the ambient air consists of a high grade of air pollutions such as: sand, salt, oil, and other contaminations which affect the engine's performance. The high grade of air pollutions results in compressor fouling quite frequently, thus the service personnel needs to shut down the gas turbine for maintenance, at least, each quarter. In Scott (1986), a compressor wash is suggested when the efficiency of the compressor has deteriorated $3 \%$. At SIT, a common procedure is to wash the compressor before the isentropic efficiency and the mass flow have deteriorated $3 \%$ from the nominal base line. The focus in the present paper is not to decide a precise limit when it is time to wash the compressor. Instead, a wash condition interval is chosen based on the value given by Scott (1986) and the experimental data given by SIT where the fouling increases with the decreased performance. Thus, the wash condition interval is chosen to be $2.0-3.5 \%$ for the efficiency health parameter $\Delta \eta_{C_{1}}$ and $2.0-3.5 \%$ for the flow health parameter $\Delta \Gamma_{C_{1}}$. The band gap is 1.5 percent points for the both cases. In the experimental data, the compressor is washed at day: 15,56 , and 144 which the estimation of performance deterioration shown in, e.g., Fig. 9.12 (blue line) indicates. Since sensor and actuator faults may affect the performance parameters in the test quantity $T_{0}$, it is desirable if these faults 
can be detected and isolated as soon as possible to avoid unnecessary compressor washes, or a compressor which is not washed when it is significantly fouled. If the floor limit of the wash condition interval is increased, the time to detect the fault is reduced, and vice versa. Thus, it is a trade of between the ability to detect the component deterioration and the fault detectability of the FDI-system.

The evaluation of the FDI-system is divided into three separate parts: (i) investigate the fault detectability and isolability of the system, (ii) investigate the performance estimation of deterioration in test quantity $T_{0}$ when fault $f_{i}$ is decoupled, and (iii) investigate how well the test quantity $T_{0}$ perform when a step fault is detected, isolated, and compensated for in the signal (fault tolerant supervision). In the investigation, the FDI-system is tuned so there are no false alarms generated using the experimental data sequences.

\subsubsection{Evaluation 1: Fault Detection and Isolation}

The fault detectability and isolability of the FDI-system are investigated using two types of fault signatures which propagate: (i) immediately (step fault), and (ii) slowly (ramp fault) to a specific fault amplitude. A fault that deviates much from the performance deterioration is easier to isolate. Thus, a step fault is easier than a ramp fault to isolate (for a given fault amplitude) since it is assumed that the performance deterioration propagates slowly, i.e., rapid changes in the health parameters are not allowed. For example, it is possible to have a fault which has a major impact on the compressor efficiency and behaves similar as a deteriorated component. This results in a fault which makes it difficult to discriminate between the fault and the fouling of the compressor. For the fault detectability of the two extreme cases, the opposite is true. It is easier to detect a ramp fault then a step fault since it takes some time for the estimated faults to reach the thresholds which trigger the alarm. How fast the fault is detected depends on the actual tuning of the CGEKFs.

\section{Step Faults}

The step fault detectability and isolability of FDI-system are evaluated using the fault amplitudes given in Tab. 9.8. The fault is injected at day 60, which are some days after the compressor is washed. The fault is injected directly after the compressor wash to have enough time to detect and isolate the fault before the compressor should be washed due to fouling. The sign of the injected fault also affect the FDI performance as shown in the simulation study in Sec. 9.5.

For each fault mode, the detection and isolation times are shown in the Tab. 9.8. For the isolation time, the diagnosis chosen by the FDI-system is also the correct diagnosis. Almost all faults are detected immediately where also all test quantities $T_{i}$ are triggered which means that the behavior can not be described by the isolation logic. After some time, the amplitude of the estimated fault $f_{i}$ by the FDI-system is in the same range as the injected fault which results in a fault which is decoupled in test quantity $T_{i}$ and $T_{0}$. This gives the time values in the right column in the table. All faults are uniquely isolable except for the fault modes: $F_{t_{7}}$ and $F_{P_{A}}$, where it is, e.g., not possible to isolate a fault 
Table 9.8: The detection and isolation time when a step fault is introduced in the signals. Fault modes $F_{t_{7}}$ and $F_{P_{A}}$ can not be uniquely isolated with the given fault size.

\begin{tabular}{|c|c|c|c|}
\hline \multicolumn{2}{|c|}{ Step Fault } & \multirow{2}{*}{$\begin{array}{c}\text { Detection } \\
\text { Time [days] }\end{array}$} & \multirow{2}{*}{$\begin{array}{c}\text { Isolation } \\
\text { Time [days] }\end{array}$} \\
\hline Mode & Size & & \\
\hline$F_{N F}$ & - & - & - \\
\hline$F_{p_{1}}$ & $+4 \%$ & 0.0 & 1.8 \\
\hline$F_{p_{3}}$ & $+4 \%$ & 0.2 & 0.7 \\
\hline$F_{p_{8}}$ & $+4 \%$ & 0.1 & 0.3 \\
\hline$F_{t_{2}}$ & $+3 \%$ & 0.2 & 0.2 \\
\hline$F_{t_{3}}$ & $+3 \%$ & 0.3 & 1.4 \\
\hline$F_{t_{7}}$ & $+3 \%$ & 0.1 & $2.8\left(F_{t_{7}}, F_{m_{f}}\right)$ \\
\hline$F_{n_{C 1}}$ & $+5 \%$ & 0.1 & 0.6 \\
\hline$F_{n_{T 0}}$ & $+5 \%$ & 1.4 & 1.4 \\
\hline$F_{m_{f}}$ & $+5 \%$ & 0.2 & 0.2 \\
\hline$F_{P_{A}}$ & $+5 \%$ & 1.1 & $1.1\left(F_{n_{T 0}}, F_{m_{f}}, F_{P_{A}}\right)$ \\
\hline
\end{tabular}

in the sensor measuring $m_{f}$ without increasing the fault amplitude. Similar arguments where shown in the simulation study which was performed in the previous section.

\section{Ramp Faults}

To investigate how the FDI-system responds to a ramp fault, the fault amplitudes shown in Tab. 9.9 are added to the input and output signals. The fault starts at day 60, and the duration is 50 days until it reaches the maximum amplitude. If the fault is detected and isolated fast, it is an indication that a smaller fault signature can be used in this specific case, e.g., for faults in sensors measuring $p_{1}$ and $n_{C 1}$.

For each fault mode, three time values are shown in Tab. 9.9. These values are: (i) the time when the fault is detected, (ii) the time and fault modes when only three (or less) diagnoses remain in the possible diagnosis set, and (iii) the time when the fault is uniquely isolated (if possible). As shown in the table, faults in sensors measuring: $p_{1}, p_{8}, t_{2}$, and $n_{C 1}$ are easy to detect and isolate, while faults in sensors measuring: $p_{3}, t_{3}$, and $t_{7}$ are more difficult detect and isolate which also the simulation study indicated.

For the injected slow varying faults, the primary objects are to detect and isolate all faults before: (i) the compressor is washed at day 144, and (ii) the performance has degraded to a point where a compressor wash alarm is triggered. If the fault is not detected before day 144, it can be an indication that the fault has violated the compressor wash condition through compensation in the performance deterioration. On the other hand, if the compressor wash alarm is triggered before day 144, the fault has assisted the deterioration rate which results in a too early wash. As shown in Tab. 9.9, all faults except for the sensors measuring $n_{T 0}$ and $P_{A}$ are detected and isolated before the compressor is washed or the wash condition alarm is triggered. The fault modes $F_{n_{T 0}}$ and $F_{P_{A}}$ cannot be isolated uniquely since it is difficult to discriminate between a fault in the speed $n_{T 0}$ and in the power $P_{A}$ generated by the power turbine. The fault cases $F_{t_{3}}$ and $F_{t_{7}}$ which 
Table 9.9: Detection and isolation time when the faults start at day 60 and ends at day 110. The amplitudes of the ramp faults are the positive values given in Tab. 9.7.

\begin{tabular}{|c|c|c|c|c|c|}
\hline \multicolumn{2}{|c|}{ Ramp Fault } & \multirow{2}{*}{$\begin{array}{c}\text { Detection } \\
\text { Time [days] }\end{array}$} & \multicolumn{2}{|c|}{ Multiple Diagnoses } & \multirow{2}{*}{$\begin{array}{c}\text { Isolation } \\
\text { Time [days] }\end{array}$} \\
\hline Mode & Size & & Time [days] & Mode & \\
\hline$F_{N F}$ & - & - & - & All & - \\
\hline$F_{p_{1}}$ & $+4 \%$ & 2.9 & 2.9 & $F_{p_{1}}, F_{n_{T 0}}$ & 2.9 \\
\hline$F_{p_{3}}$ & $+5 \%$ & 11.5 & 26.9 & $F_{p_{3}}, F_{t_{7}}, F_{m_{f}}$ & 44.6 \\
\hline$F_{p_{8}}$ & $+4 \%$ & 7.7 & 8.0 & $F_{p_{8}}$ & 8.0 \\
\hline$F_{t_{2}}$ & $+3 \%$ & 12.9 & 13.0 & $F_{t_{2}}$ & 13.0 \\
\hline$F_{t_{3}}$ & $+3 \%$ & 24.3 & 41.3 & $F_{t_{2}}, F_{t_{3}}, F_{P_{A}}$ & 43.2 \\
\hline$F_{t_{7}}$ & $+5 \%$ & 11.3 & 29.5 & $F_{t_{7}}, F_{m_{f}}, F_{P_{A}}$ & 53.5 \\
\hline$F_{n_{C 1}}$ & $+5 \%$ & 7.5 & 7.5 & $F_{n_{C 1}}, F_{m_{f}}$ & 7.5 \\
\hline$F_{n_{T 0}}$ & $+5 \%$ & 29.3 & 40.3 & $F_{n_{T 0}}, F_{m_{f}}, F_{P_{A}}$ & - \\
\hline$F_{m_{f}}$ & $+10 \%$ & 10.5 & 14.8 & $F_{p_{3}}, F_{t_{7}}, F_{m_{f}}$ & 17.7 \\
\hline$F_{P_{A}}$ & $+10 \%$ & 14.1 & 19.2 & $F_{n_{T 0}}, F_{P_{A}}$ & - \\
\hline
\end{tabular}

are most difficult to diagnose in the case study are presented in the remainder of this section.

Fault Mode $\mathbf{F}_{\mathbf{t}_{7}}$ : A fault with a positive amplitude of $5 \%$ in the sensor measuring the exhaust temperature $t_{7}$ of the power-turbine gives a reduction in the estimated value of the mass flow throughout the compressor. As Fig. 9.11b indicates, this is compensated through a reduction in the health parameter $\Delta \Gamma_{C 1}$. The reduction in $\Delta \Gamma_{C 1}$ reduces the ability to detect the fault in the residuals, thus the fault estimate in Fig. 9.11c is instead used for fault detection.

In the figure, the health parameters $\Delta \eta_{C 1}$ and $\Delta \Gamma_{C 1}$ are shown for the faulty (red line) and non-fault (blue line) case for the test quantity $T_{0}$ (which has no extra fault parameters). In Fig. 9.11c, the introduced fault is shown together with the estimated fault by test quantity $T_{6}$ (which decouples fault $f_{6}$ ). The estimate seems to match the injected fault pretty well.

The fault is detected before the compressor wash alarm is triggered by the FDI-system, and the fault is isolated about 40 days later. Without the fault detection alarm, the FDIsystem should not satisfy the condition to find the fault before the compressor wash alarm is triggered. Since a fault alarm is generated, the service personnel have the choice to: (i) continue the operation until a unique diagnosis is stated, or (ii) see which diagnoses that are consistent with the measurements and analyze if it is possible exclude some of the remaining diagnoses. An analysis can be to, e.g., exclude fault modes that represent fault estimates which have not exceeded its threshold. Another technique is to see which diagnoses that are left, e.g., about 20 days after the fault is detected, only the diagnoses of: $F_{t_{7}}, F_{m_{f}}$, and $F_{P_{A}}$ are left in the possible set of diagnoses. It can be useful to look at the health parameters which are estimated by the tests: $T_{6}, T_{9}$, and $T_{10}$ which have not triggered, and see if some of them seem to be more likely than the others. Finally, the fault is uniquely diagnosed after about 40 days. Thus, it is not possible to isolate the fault 


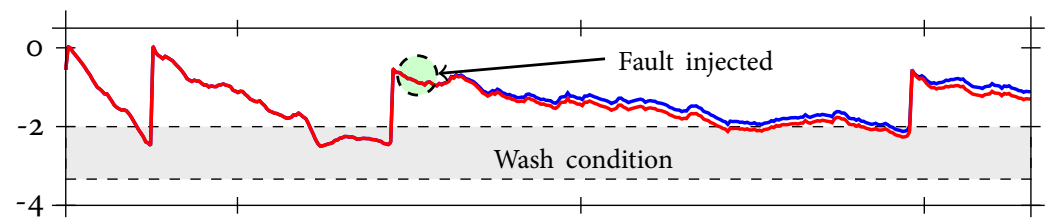

(a) $T_{0}: \Delta \eta_{C 1}[\%]$

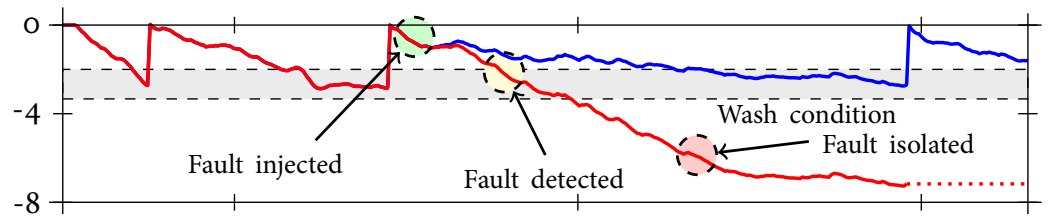

(b) $T_{0}: \Delta \Gamma_{C 1}[\%]$

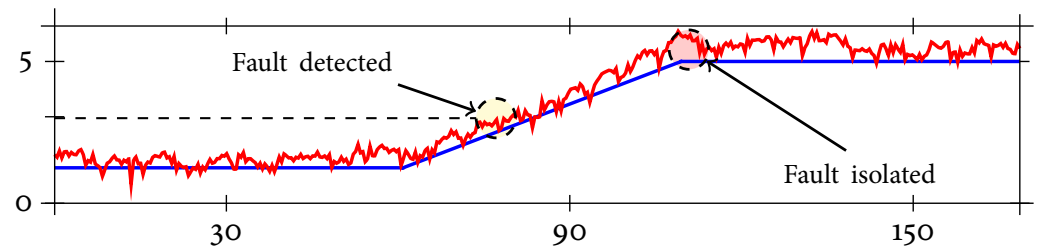

(c) $T_{6}: f_{6}$ (blue line), and $\hat{f}_{6}$ (red line) $[\%]$

Figure 9.11: Estimation of performance deviation in the compressor for the cases of: no fault (blue line), and sensor fault in $y_{6}$ (red line). The estimated (red line) and injected (blue line) fault are shown in (c). The sensor fault affects the mass flow parameter at most.

uniquely before a wash condition is triggered. But the operator knows that a fault is present before the wash condition alarm is triggered.

Fault Mode $\mathbf{F}_{\mathbf{t}_{3}}$ : A fault with a positive amplitude of $3 \%$ in the sensor measuring the discharge temperature $t_{3}$ of the compressor gives a reduction in the estimated value of the isentropic efficiency of the compressor. This is compensated through a reduction in the health parameter $\Delta \eta_{C 1}$ as Fig. 9.12a indicates.

The fault is detected in the lower limit of the wash condition interval where the $\Delta \eta_{C 1}$ parameter suggests a compressor wash. The fault is isolated about 20 days after the fault is detected. Since the fault is detected in the lower limit of the interval, the specific fault size can be misinterpreted as a fouled compressor. Since the fault is not detected at this point, it is not known if the $\Delta \eta_{C 1}$ parameter represents the true deterioration or not which is a disadvantage. If it is assumed that the both health parameters have to deteriorate approximately in the same magnitude, a red flag could be raised when only one of the two health parameters suggests a compressor wash. With this information, it is possible to detect the fault earlier. 


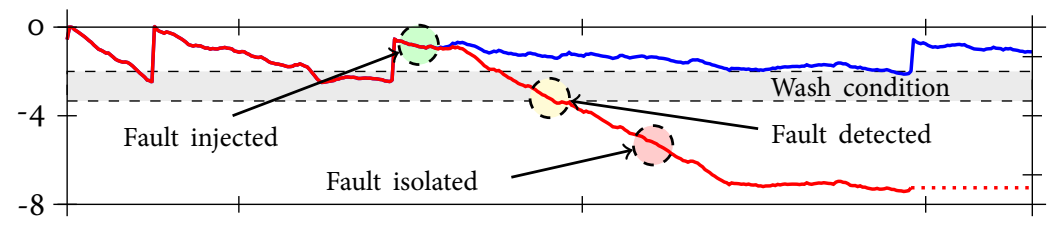

(a) $T_{0}: \Delta \eta_{C 1}[\%]$

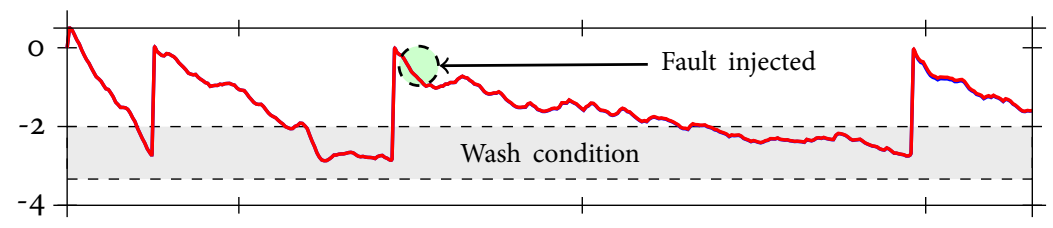

(b) $T_{0}: \Delta \Gamma_{C 1}[\%]$

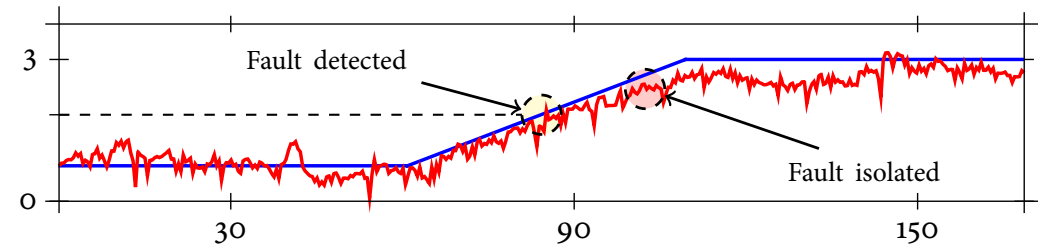

(c) $T_{5}: f_{5}$ (blue line), and $\hat{f}_{5}$ (red line) $[\%]$

Figure 9.12: Estimation of performance deviation in the compressor for the cases of: no fault (blue line), and sensor fault in $y_{5}$ (red line) when the test $T_{0}$ is utilized. The estimated (red line) and injected (blue line) fault are shown in (c). The sensor fault affects the efficiency parameter at most.

\subsubsection{Evaluation 2: Supervision Based on Fault Compensation in Test Quantity $T_{0}$}

Before the results of the fault tolerant supervision technique are presented, it is interesting to see how the test quantity $T_{0}$ responds to fault free data where an estimation of fault $f_{i}$ is used to compensate for a potential fault in the signals. The evaluation shows in some sense how good the health estimation is when one sensor or actuator is removed, i.e., the fault is decoupled. Here, the fault free case is presented and the estimation of the fault should ideally be zero. The evaluation is performed as follows: (i) assume that the diagnosis based on fault mode $F_{i}$ is determined, (ii) use the estimated fault $f_{i}$ from the test quantity $T_{i}$ to compensate for the fault in the test quantity $T_{0}$, and (iii) compare the health estimations with the non-faulty case. The key step here is to get a good estimation of the fault, i.e., a small fault estimation error gives a small spread between the health estimations for different fault cases. When the estimated fault is not zero, it will influence the health parameters in $T_{0}$ exactly in the same way as a fault.

The fault cases $F_{t_{3}}$ and $F_{t_{7}}$ have the largest deviation in health parameters and fault estimations. These two fault cases are therefore shown in Fig. 9.13. With the chosen wash 


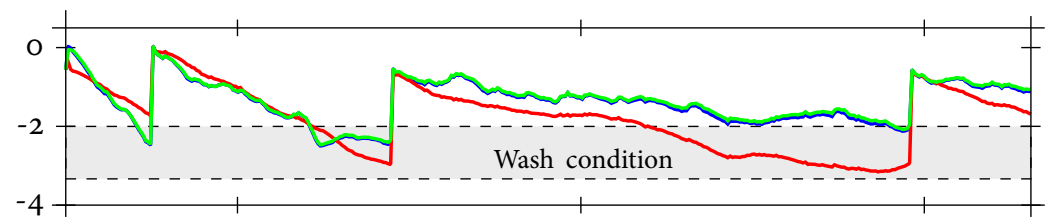

(a) $T_{0}: \Delta \eta_{C 1}[\%]$

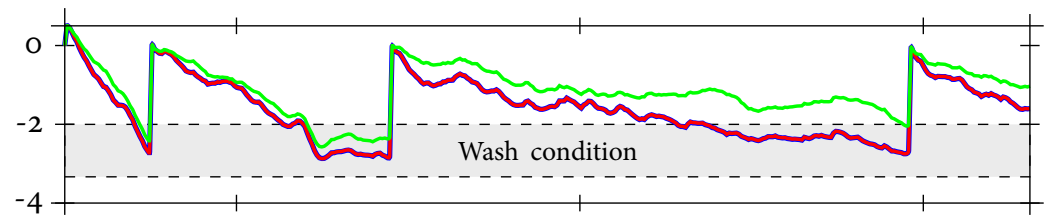

(b) $T_{0}: \Delta \Gamma_{C 1}[\%]$

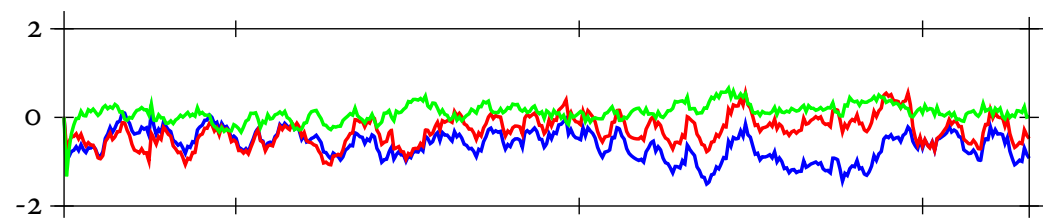

(c) $T_{0}: \Delta \eta_{T 1}[\%]$

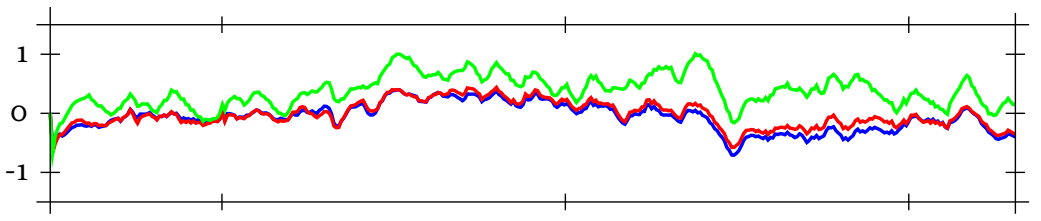

(d) $T_{0}: \Delta \Gamma_{T 1}[\%]$

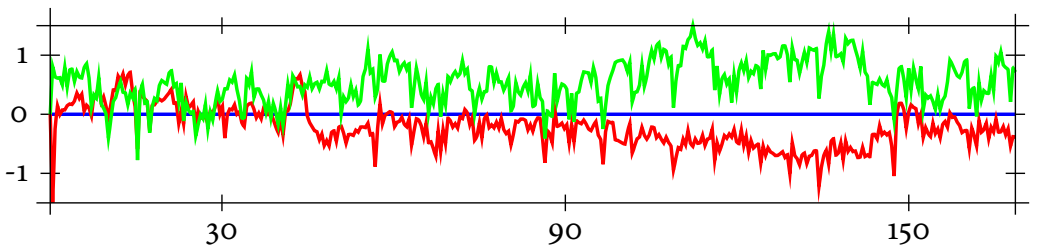

(e) $T_{5}, T_{6}: \hat{f}[\%]$

Figure 9.13: Supervision of health for the fault free case (blue line) together with estimation of faults based on: test quantity $T_{5}$ (red line), and test quantity $T_{6}$ (green line). For all three cases, fault free data is used and the estimations are performed using test quantity $T_{0}$. In the last figure, the estimated fault $\hat{f}_{i}$ is viewed which should be zero in an ideal case. 
condition interval, the two health parameters for the compressor are inside the chosen interval. When a fault in the sensor measuring the compressor discharge temperature $t_{3}$ is present, the estimation of efficiency degrades faster than for the non-faulty sequence.

Since the sensor which measuring the compressor discharge temperature is decoupled and this sensor is important for the efficiency calculation, it is not surprising that the estimation of efficiency differs from the fault free case. But when this sensor is decoupled, it is possible to supervise the performance of the compressor using the fault tolerant supervision system.

\subsubsection{Evaluation 3: Fault Tolerant Supervision of Performance}

When a fault is diagnosed, the idea is to compensate for the fault in the measurements. The compensation is done using the estimation of fault $f_{i}$ which is calculated by the test quantity $T_{i}$. Thus, when the correct diagnosis is stated, the fault is decoupled in test quantity $T_{0}$.

The fault tolerant FDI-system is evaluated using a step fault with an amplitude of $3 \%$ in the sensor measuring temperature $t_{3}$. The fault is injected at day 60 , and is immediately detected by the FDI-system at the same time as all the test quantities alarm. After about two days the correct diagnosis is stated and the fault parameter in $T_{5}$ has estimated the injected fault. The $\Delta \eta_{C 1}$ parameter is shown in Fig. 9.14 for the three cases where the parameter is: (i) compensated for the fault when it is isolated (blue line), (ii) based on

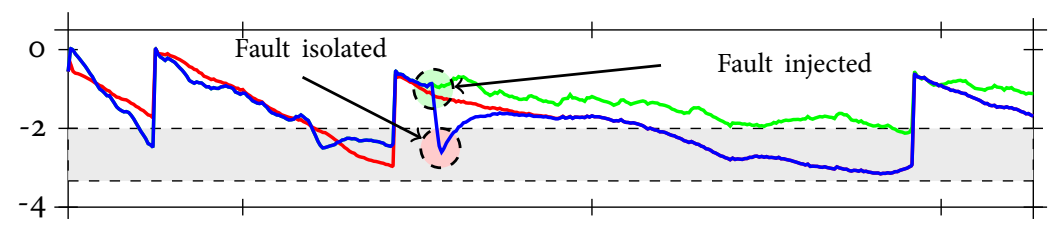

(a) $T_{0}: \Delta \eta_{C 1}[\%]$

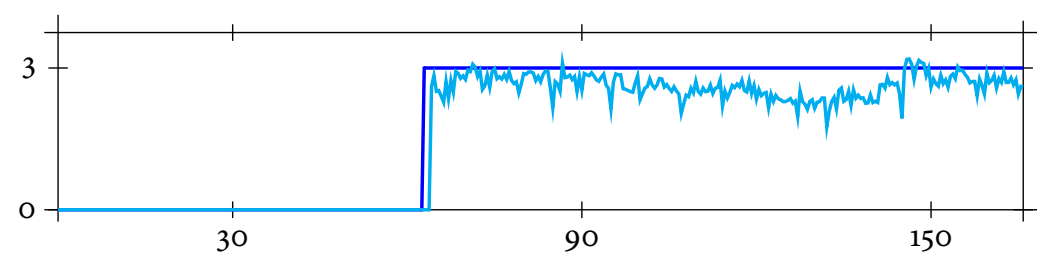

(b) $T_{5}: f_{5}$ (blue line), $\hat{f}_{\text {in }}$ (cyan line) $[\%]$

Figure 9.14: Fault tolerant supervision of the $\Delta \eta_{C 1}$ parameter (blue line), when fault $f_{5}$ is injected. The estimation is compared to the fault free estimation (green line), and the estimation of $T_{0}$ based on fault estimation of $T_{5}$ (red line) in the fault free case. The measurement signal $y_{5}$ is compensated with the signal $\hat{f}_{i}$ to get the fault tolerant supervision of the $\Delta \eta_{C 1}$ parameter.

fault free data (green line), and (iii) based on the fault free case together the fault which is estimated by the test $T_{5}$ (red line) are all shown. In the second figure, the injected and 
estimated fault is shown. For this test case, it is easy to detect the fault and also isolate the fault before the wash condition alarm is triggered.

\subsubsection{Summary}

The experimental case studies show that all considered fault modes can be detected where also the most of them are possible to isolate from each other. The faults which are most difficult to isolate are faults in the sensors measuring discharge pressure $p_{3}$ and temperature $t_{3}$ after the compressor, and exhaust gas temperature $t_{7}$. Faults in the two actuator signals are also difficult to isolate uniquely from each other, but can be isolated from the measurement sensors. Faults which behave like a step is easier to isolate than a slow drifting bias fault. But the detection performance is the same for the two fault types given the same amplitude. To increase the isolation performance, an upper and lower limit of the health parameters is considered. If these limits are not considered, a slow drifting bias fault can behaves similar to a fouled component.

\subsection{Conclusion}

In this work, an FDI-system for an industrial gas turbine is designed using a model based approach. The FDI-system consists of: (i) a bank of constant gain extended Kalman filters, (ii) a fault detection component, (iii) an isolation component, (iv) and a compressor wash detection component. For each filter, a fault is decoupled. The filter where the fault is decoupled can be used to supervise the performance in case of a fault, and the other filters should have large estimation errors and triggered. The FDI-system is evaluated using: (i) data generated from a simulation platform, and (ii) experimental data collected from a gas turbine site. To receive better isolation performance, a lower and an upper limit of the health parameters are introduced. These limits have to be adjusted to the actual performance condition of the engine. A tighter band limit can result in better isolation performance since the fault signature is more visible in the residuals. When the band is too tight, the natural performance deterioration cannot be captured. Thus, it is a trade of between fault isolation and the estimation of health. The fault detection performance is not dependent of the chosen interval limits since all faults are observable and thus estimated correctly by the right test quantity.

After the investigation, the conclusions are: (i) all considered faults are detectable, (ii) faults in all eight output signals can be isolated correctly from each other when the fault amplitude is $5 \%$, (iii) component deteriorations are compensated using health parameters, and (iv) a fouled compressor can be detected even when a single fault is present. 



\section{Chapter 10}

\section{Conclusion}

When a diagnosis and supervision system is designed using model based diagnosis techniques, an important factor which affects the fault detection and false alarm probability is the accuracy of the embedded models. To have good diagnosis performance, it is valuable to have diagnosis test quantities based on models which have high accuracy since this is also reflected in the diagnosis decision. Thus, when designing a diagnosis and supervision system of an industrial gas turbine it is crucial to consider: (i) physical relationships such as mass and energy balances, (ii) thermodynamic gas properties such as enthalpy, heat capacity, and entropy, and (iii) performance characteristics of the gas turbine components. To simplify the construction of the gas turbine model, it is suitable to collect these aspects in a component library, here called GTLib, using an equation based modeling language. The gas turbine model is then built using the predefined components in GTLib. In GTLib, the thermodynamic gas properties rely on the the NASA Glenn Coefficients which are encapsulated by the gas model. The performance characteristics rely on look up tables, which are based on measurements performed by the manufacturer. The benefit with using GTLib is the reduction in model equations and state variables in the gas turbine model compared to the original gas turbine model developed by SIT. The reduction depends largely on the usage of the air/fuel ratio concept instead of the mass fraction of substances in the gas mixture as proposed in the original model. The constructed gas turbine model can be used for performance analysis and as a base (diagnosis model) when diagnosis tests are extracted. In the diagnosis model, a number of health parameters are introduced to capture performance deterioration in the compressor, and compressor-turbine. In the same way, faults in components can be introduced in the diagnosis model. The component faults can represent, e.g., leakages or increased friction in bearings. Since an equation based modeling framework is considered, the diagnosis model is in an index-2 DAE form and cannot be treated as an ordinary state space model without modifications. It is also shown that unobservable state variables are present, but these modes disappear when the over-determined part of the diagnosis model is considered. The over-determined part is determined using 
structural methods. The equations which represent the over-determined part are implemented as CGEKF based test quantities for a chosen operational point and for a suitable choice of the noise matrices $Q$ and $R$.

In the second part of the thesis, the methodology is evaluated using case studies. In the first part, the CGEKFs are evaluated with focus on: (i) simulated data generated from the reference simulation platform when the ambient conditions and demanded power are varied, (ii) the flame temperature $T_{f}$ which is an important parameter for the controller, and (iii) the performance deterioration due to compressor fouling. The conclusion from the simulation study is that large deviations in the ambient conditions affect the estimated health parameter in the same range as a fouled compressor, i.e., a deterioration of 1-2\%. To minimize the error depending on the change in ambient conditions, the observers can compensate for the deviation in ambient conditions. In the last two case studies, experimental data from a Middle East site are investigated. The environment conditions at the Middle East site are tough due to contaminants in the air, which results in frequently compressor washes. Using the estimated deterioration, a decision of a fouled compressor should be taken. The investigation indicates that it is possible to use the test in a FDI-system to detect a fouled compressor since the deterioration of the compressor decreases about $2 \%$ between the compressor washes. Since the interval and offset of the deterioration are the same independently of the winter or summer period, it is possible to have static thresholds for fouling detection in the FDI-system. The flame temperature is estimated at the same time as the test quantity compensates for the deteriorations in the compressor $\mathrm{C} 1$ and turbine $\mathrm{T} 1$.

Finally, to detect and isolate faults in actuators and sensors an FDI-system which consists of a number of diagnosis test quantities is designed. The FDI-system can be used to supervise the performance also when a fault has occurred. Slowly drifting bias faults are more difficult than abrupt faults to diagnose, since the health parameters capture the fault in some sense. Thus, it is difficult to discriminate the fault signature in the residuals from the non-faulty case since measurement noise is present. With the constructed FDI-system, the performance to detect a slowly drifting bias fault is the same (or little better) as for an abrupt fault. To increase the fault isolation, a lower and an upper limit of the health parameters are introduced. After the performed case studies, the conclusions are: (i) all faults are detectable, (ii) faults in all eight output signals can be isolated correctly from each other when the fault amplitude is $5 \%$, (iii) component deteriorations are compensated using health parameters, and (iv) a fouled compressor can be detected even when a single fault is present. 


\section{References}

G. F. Aker and H. I. H. Saravanamuttoo. Predicting gas turbine performance degradation due to compressor fouling using computer simulation techniques. Journal of Engineering for Gas Turbines and Power, 111(2):343-350, 1989. doi:10.1115/1.3240259. URL http: //link.aip.org/link/?GTP/111/343/1.

Uri M. Ascher and Linda R. Petzold. Computer Methods for Ordinary Differential Equations and Differential-Algebraic Equations. Society for Industrial and Applied Mathematics, Philadelphia, PA, USA, 1st edition, 1998. ISBN 0898714125.

J. Bird and W. Grabe. Humidity effects on gas turbine performance. In International Gas Turbine and Aeroengine Congress and Exposition, Orlando, FL, 1991.

M. Blanke, M. Kinnaert, J. Lunze, and M. Staroswiecki. Diagnosis and Fault-Tolerant Control. Springer, 2003.

S. Borguet and O. Léonard. A sensor-fault-tolerant diagnosis tool based on a quadratic programming approach. Journal of Engineering for Gas Turbines and Power, 130(2): 021605, 2008. doi:10.1115/1.2772637. URL http://link . aip.org/link/?GTP/130/ 021605/1.

Gary L. Borman and Kenneth W. Ragland. Combustion Engineering. McGraw-Hill, 1998. ISBN 0-07-006567-5.

Olaf Brekke, Lars E. Bakken, and Elisabet Syverud. Compressor fouling in gas turbines offshore: Composition and sources from site data. ASME Conference Proceedings, 2009 (48869):381-391, 2009. doi:10.1115/GT2009-59203. URL http://link.aip.org/ link/abstract/ASMECP/v2009/i48869/p381/s1.

Arden L. Buck. New equations for computing vapor pressure and enhancement factor. Journal of Applied Meterology, 20:1529, 1981.

E. Buckingham. On physically similar systems; illustrations of the use of dimensional equations. Phys. Rev., 4(4):345-376, Oct 1914. doi:10.1103/PhysRev.4.345. 
Francesco Casella, Martin Otter, Katrin Proelss, Christoph Richter, and Hubertus Tummescheit. The modelica fluid and media library for modeling of incompressible and compressible thermo-fluid pipe networks. The 5th International Modelica Conference $2006,2006$.

Malcolm W. Chase, Jr, editor. NIST-JANAF Thermochemical Tables, volume Part 1 and Part 2 of Journal of Physical and Chemical Reference Data. American Institute of Physics, 500 Sunnyside Boulevard, Woodbury, New York, fourth edition, 1998. ISBN 1-56396-831-2.

J. Chen and R.J Patton. Robust Model-Based Fault Diagnosis for Dynamic Systems. Kluwer Academic Publishers, 1999. ISBN 0-7923-8411-3.

P. Dewallef, C. Romessis, O. Léonard, and K. Mathioudakis. Combining classification techniques with kalman filters for aircraft engine diagnostics. Journal of Engineering for Gas Turbines and Power, 128(2):281-287, 2006. doi:10.1115/1.2056507. URL http: //link.aip.org/link/?GTP/128/281/1.

I. S. Diakunchak. Performance deterioration in industrial gas turbines. Journal of Engineering for Gas Turbines and Power, 114(2):161-168, 1992. doi:10.1115/1.2906565. URL http://link. aip.org/link/?GTP/114/161/1.

S.L. Dixon and Cesare Hall. Fluid Mechanics and Thermodynamics of Turbomachinery. Elsevier, 6th edition edition, 2010. ISBN 978-1-85617-793-1.

D. L. Doel. Temper-a gas-path analysis tool for commercial jet engines. Journal of Engineering for Gas Turbines and Power, 116(1):82-89, 1994. doi:10.1115/1.2906813. URL http://link . aip.org/link/?GTP/116/82/1.

D. L. Doel. Interpretation of weighted-least-squares gas path analysis results. Journal of Engineering for Gas Turbines and Power, 125(3):624-633, 2003. doi:10.1115/1.1582492. URL http://link. aip.org/link/?GTP/125/624/1.

A. L. Dulmage and N. S. Mendelsohn. Coverings of bipartite graphs. Canadian Journal of Mathematics, 10:517-534, 1958.

Thomas D. Eastop and Allan McConkey. Applied Thermodynamics for Engineering Technologists. Prentice Hall, 5th edition, 1993. ISBN 0-582-09193-4.

Lars Eriksson. CHEPP - A chemical equilibrium program package for matlab. In Modeling of Spark Ignition Engines, number 2004-01-1460 in SAE Technical paper series SP-1830, 2004. doi:10.4271/2004-01-1460.

Erik Frisk, Mattias Krysander, and Jan Åslund. Sensor placement for fault isolation in linear differential-algebraic systems. Automatica, 45(2):364-371, 2009. doi:10.1016/j.automatica.2008.08.013.

Peter Fritzson. Principles of Object-Oriented Modeling and Simulation with Modelica 2.1. Wiley, 2004 . 
Ranjan Ganguli and Budhadipta Dan. Trend shift detection in jet engine gas path measurements using cascaded recursive median filter with gradient and laplacian edge detector. Journal of Engineering for Gas Turbines and Power, 126(1):55-61, 2004. doi:10.1115/1.163540o. URL http://link. aip.org/link/?GTP/126/55/1.

Tony Giampaolo. Gas Turbine Handbook: Principles and Practice. The Fairmont Press, 4th edition edition, 2009. ISBN o-88173-613-9.

Sanford Gordon and Bonnie J. McBride. Computer program for calculation of complex chemical equilibrium compositions, rocket performance, incident and reflected shocks, and chapman-jouguet detonations. interim revision, march 1976. Technical Report SP-273, National Aeronautics and Space Administration (NASA), 1971. URL http: //ntrs .nasa.gov/search $\cdot$ jsp?print=yes\&R=19780009781.

Sanford Gordon and Bonnie J. McBride. Computer program for calculation of complex chemical equilibrium compositions and applications i. analysis. Technical Report RP-1311, National Aeronautics and Space Administration (NASA), 1994. URL http: //www.grc.nasa.gov/WWW/CEAWeb/RP-1311.htm.

Fredrik Gustafsson. Adaptive filtering and change detection / Fredrik Gustafsson. Chichester : Wiley, cop. 2000, 2000. ISBN 0471492876.

A. A. El Hadik. The impact of atmospheric conditions on gas turbine performance. Journal of Engineering for Gas Turbines and Power, 112(4):590-596, 1990. doi:10.1115/1.2906210. URL http://link . aip.org/link/?GTP/112/590/1.

E. Hairer, S. P. Norsett, and G. Wanner. Solving Ordinary Differential Equations II: Stiff and Differential-Algebraic Problems. Springer, Berlin, 2nd revised edition edition, 1991.

M. Härefors. Application of $\mathrm{h}_{\infty}$ robust control to the RM12 jet engine. Control Engineering Practice, 5(9):1189 - 1201, 1997. ISSN 0967-0661. doi:10.1016/So967o661(97)84358-4. URL http://www.sciencedirect.com/science/article/ pii/S0967066197843584.

R. Hermann and A. Krener. Nonlinear controllability and observability. Automatic Control, IEEE Transactions on, 22(5):728 - 740, oct 1977. ISSN oo18-9286. doi:10.1109/TAC.1977.1101601.

John B. Heywood. Internal Combustion Engine Fundamentals. McGraw-Hill series in mechanical engineering. McGraw-Hill, 1988. ISBN 0-07-100499-8.

J. H. Horlock. Advanced Gas Turbine Cycles. Pergamon, revised edition edition, 2007.

A Idebrant and Lennart Näs. Gas Turbine Applications using Thermofluid. In Peter Fritzson, editor, Proceedings of the 3rd International Modelica Conference, pages 359366. The Modelica Association, May 2003.

Thomas Kailath. Linear Systems. Prentice-Hall, Inc, Englewood Cliffs, New Jersey o7632, 1980. 
Thomas Kailath, Ali H. Sayed, and Babak Hassibi. Linear Estimation. Prentice-Hall, Inc, Upper Saddle River, New Jersey 07458, 2 edition, 2000.

Takahisa Kobayashi and Donald L. Simon. Application of a Bank of Kalman Filters for Aircraft Engine Fault Diagnostics. NASA/TM-2003-212526, 2003. URL http://ntrs . nasa. gov/search $\cdot$ jsp?print=yes\&R=20030067984.

Takahisa Kobayashi and Donald L. Simon. Evaluation of an enhanced bank of kalman filters for in-flight aircraft engine sensor fault diagnostics. Journal of Engineering for Gas Turbines and Power, 127(3):497-504, 2005. doi:10.1115/1.1850505. URL http:// link. aip.org/link/?GTP/127/497/1.

Takahisa Kobayashi, Donald L. Simon, and Jonathan S. Litt. Application of a Constant Gain Extended Kalman Filter for In-Flight Estimation of Aircraft Engine Performance Parameters. NASA/TM-2005-213865, 2005. URL http://ntrs .nasa.gov/search. jsp?print=yes\&R=20050216398.

Milton G. Kofskey and William J. Nusbaum. Performance evaluation of a two-stage axial-flow turbine for two values of tip clearance. Technical Report TN D-4388, National Aeronautics and Space Administration (NASA), 1968.

Mattias Krysander and Erik Frisk. Sensor placement for fault diagnosis. IEEE Transactions on Systems, Man, and Cybernetics - Part A: Systems and Humans, 38(6):1398-1410, 2008.

Mattias Krysander, Jan Åslund, and Mattias Nyberg. An efficient algorithm for finding minimal over-constrained sub-systems for model-based diagnosis. IEEE Transactions on Systems, Man, and Cybernetics - Part A: Systems and Humans, 38(1), 2008.

R. Kurz and K. Brun. Degradation in gas turbine systems. Journal of Engineering for Gas Turbines and Power, 123(1):70-77, 2001. doi:10.1115/1.1340629. URL http: //link.aip.org/link/?GTP/123/70/1.

Rainer Kurz, Klaus Brun, and Meron Wollie. Degradation effects on industrial gas turbines. Journal of Engineering for Gas Turbines and Power, 131(6):062401, 2009. doi:10.1115/1.3097135. URL http://link . aip.org/link/?GTP/131/062401/1.

Emil Larsson. Diagnosis and Supervision of Industrial Gas Turbines. Technical report, Department of Electrical Engineering, 2012. LiU-TEK-LIC-2012:13, Thesis No. 1528, http://urn.kb.se/resolve?urn=urn:nbn:se:liu:diva-75985.

Emil Larsson, Jan Åslund, Erik Frisk, and Lars Eriksson. Fault isolation for an industrial gas turbine with a model-based diagnosis approach. ASME Conference Proceedings, 2010(43987):89-98, 2010. doi:10.1115/GT2010-22511.

Emil Larsson, Jan Åslund, Erik Frisk, and Lars Eriksson. Health Monitoring in an Industrial Gas Turbine Application by Using Model Based Diagnosis Techniques. ASME Conference Proceedings, 2011(54631):487-495, 2011. doi:10.1115/GT2011-46825. 
Emil Larsson, Jan Åslund, Erik Frisk, and Lars Eriksson. Fault Tolerant Supervision of an Industrial Gas Turbine. ASME Conference Proceedings, 2013(55188), 2013. doi:10.1115/GT2013-95727.

Emil Larsson, Jan Åslund, Erik Frisk, and Lars Eriksson. Gas turbine modeling for diagnosis and control. Journal of Engineering for Gas Turbines and Power, 136(7):17 pages, July 2014a. doi:10.1115/1.4026598.

Emil Larsson, Jan Åslund, Erik Frisk, and Lars Eriksson. Diagnosis and fault tolerant supervision of industrial gas turbines. Submitted for journal publication, 2014b.

Jonathan S. Litt. An optimal orthogonal decomposition method for kalman filter-based turbofan engine thrust estimation. Journal of Engineering for Gas Turbines and Power, 130(1), 2007. doi:10.1115/1.2747254.

R H Luppold, J R Roman, G W Gallops, and L J Kerr. Estimating in-flight engine performance variations using kalman filter concepts. In 25th Joint Propulsion Conference, Monterey, USA, 1989. 2584-AIAA.

R. Martínez-Guerra, R. Garrido, and A. Osorio-Miron. The fault detection problem in nonlinear systems using residual generators. IMA Journal of Mathematical Control and Information, 22(2):119-136, 2005. doi:10.1093/imamci/dnio1o.

K. Mathioudakis and T. Tsalavoutas. Uncertainty reduction in gas turbine performance diagnostics by accounting for humidity effects. Journal of Engineering for Gas Turbines and Power, 124(4):801-808, 2002. doi:10.1115/1.1470485.

S. Mattsson and G. Söderlind. Index reduction in differential-algebraic equations using dummy derivatives. SIAM Journal on Scientific Computing, 14(3):677-692, 1993. doi:10.1137/o914043. URL http://epubs.siam.org/doi/abs/10.1137/0914043.

Bonnie J. McBride and Sanford Gordon. Computer program for calculating and fitting thermodynamic functions. Technical Report RP-1271, National Aeronautics and Space Administration (NASA), 1992. URL http://www.grc.nasa.gov/WWW/ CEAWeb/RP-1271.htm.

Bonnie J. McBride, Michael J. Zehe, and Sanford Gordon. Nasa glenn coefficients for calculating thermodynamic properties of individual species. Technical Report TP-2002-211556, National Aeronautics and Space Administration (NASA), 2002. URL http://www.grc.nasa.gov/WWW/CEAWeb/TP-2002-21556.htm.

C.B. Meher-Homji. Compressor and hot section fouling in gas turbines - causes and effects. In Industrial Energy Conference, Houstion, TX, 1987.

Walter C. Merrill, John C. Delaat, and William M. Bruton. Advanced detection, isolation, and accommodation of sensor failures - real-time evaluation. Journal of Guidance, Control, and Dynamics, 11(6):517-526, 1988. doi:10.2514/3.20348. 
Modelica Association. The Modelica Language Specification version 3.0, 2007. URL http://www.modelica.org/.

E. Naderi, N. Meskin, and K. Khorasani. Nonlinear fault diagnosis of jet engines by using a multiple model-based approach. Journal of Engineering for Gas Turbines and Power, 134(1), 2011. doi:10.1115/1.4004152.

H. Nijmeijer and T.I. Fossen. New directions in nonlinear observer design. Lecture notes in control and information sciences. Springer, 1999. ISBN 9781852331344.

Ramine Nikoukhah. Innovations generation in the presence of unknown inputs: Application to robust failure detection. Automatica, 30(12):1851-1867, 1994. ISSN ooo5-1098. doi:10.1016/0005-1098(94)90047-7.

Per Öberg. A DAE Formulation for Multi-Zone Thermodynamic Models and its Application to CVCP Engines. $\mathrm{PhD}$ thesis, Linköpings universitet, 2009. URL http: //www.fs.isy.liu.se/Publications/PhD/09_PhD_1257_PO.pdf.

E.S. Page. Continuous inspection schemes. Biometrika, 41:100-115, 1954.

Constantinos C. Pantelides. The consistent initialization of differential-algebraic systems. SIAM J. Sci. Stat. Comput., 9(2):213-231, 1988.

R.J. Patton and M. Hou. Design of fault detection and isolation observers: A matrix pencil approach. Automatica, 34(9):1135 - 1140, 1998. ISSN 0005-1098. doi:10.1016/Sooo51098(98)00043-o.

Randal T. Rausch, Kai F. Goebel, Neil H. Eklund, and Brent J. Brunell. Integrated inflight fault detection and accommodation: A model-based study. Journal of Engineering for Gas Turbines and Power, 129(4):962-969, 2007. doi:10.1115/1.2720517. URL http: //link. aip.org/link/?GTP/129/962/1.

M. Safonov and M. Athans. Robustness and computational aspects of nonlinear stochastic estimators and regulators. Automatic Control, IEEE Transactions on, 23(4):717 - 725, aug 1978. ISSN o018-9286. doi:10.1109/TAC.1978.1101825.

H.I.H. Saravanamuttoo, G.F.C. Rogers, and H. Cohen. Gas Turbine Theory. Longman, 5th edition edition, 2001. ISBN 0-13-015847-X.

J.N. Scott. Reducing turbo machinery operation costs with regular performance testing. In International Gas Turbine Conference and Exhibit, Dusseldorf, Germany, 1986.

R. Shields and J. Pearson. Structural controllability of multiinput linear systems. Automatic Control, IEEE Transactions on, 21(2):203 - 212, apr 1976. ISSN oo18-9286. doi:10.1109/TAC.1976.1101198.

S. Simani and Ron J. Patton. Fault diagnosis of an industrial gas turbine prototype using a system identification approach. Control Engineering Practice, 16(7):769 - 786, 2008. ISSN 0967-0661. doi:http://dx.doi.org/10.1016/j.conengprac.2007.08.009. 
Dan Simon and Donald L. Simon. Aircraft turbofan engine health estimation using constrained kalman filtering. Journal of Engineering for Gas Turbines and Power, 127 (2):323-328, 2005. doi:10.1115/1.1789153.

Donald L. Simon and Jeffrey B. Armstrong. An integrated approach for aircraft engine performance estimation and fault diagnostics. Journal of Engineering for Gas Turbines and Power, 135(7), 2013. doi:10.1115/1.4023902.

Donald L. Simon, Jeff Bird, Craig Davison, Al Volponi, and R. Eugene Iverson. Benchmarking gas path diagnostic methods: A public approach. ASME Conference Proceedings, 2008(43123):325-336, 2008. doi:10.1115/GT2008-51360. URL http: //link.aip.org/link/abstract/ASMECP/v2008/i43123/p325/s1.

Gregory N. Stephanopoulos, Aristos A. Aristidou, and Jens Nielsen. Metabolic Engineering: Principles and Methodologies. Academic Press, 1 edition, 1998. ISBN 0126662606. URL http: //www . worldcat .org/isbn/0126662606.

N. Sugiyama. System identification of jet engines. Journal of Engineering for Gas Turbines and Power, 122(1):19-26, 2000. doi:10.1115/1.483172.

Carl Svärd and Mattias Nyberg. Observer-based residual generation for linear differential-algebraic equation systems. In IFAC World Congress, Seoul, Korea, 2008.

Michael Tiller. Introduction to Physical Modeling with Modelica. Kluwer Academic Publishers, Norwell, MA, USA, 2001. ISBN 0792373677.

Stephen R. Turns. Thermodynamics : concepts and applications. Cambridge University Press, Cambridge, 2006. ISBN 978-052-185-242-1.

L. A. Urban. Gas Turbine Engine Parameter Interrelationships. Windsor Locks, 1969.

L. A. Urban. Gas path analysis applied to turbine engine condition monitoring. Journal of Aircraft, 10(7):400-406, 1972.

L. A. Urban and A. J. Volponi. Mathematical methods of relative engine performance diagnostics. Journal of Aerospace, 1992. doi:10.4271/922048.

A. J. Volponi. Sensor error compensation in engine performance diagnostics. ASME Conference Proceedings, 1994(58), 1994.

A. J. Volponi. Gas turbine parameter corrections. Journal of Engineering for Gas Turbines and Power, 121(4):613-621, 1999. doi:10.1115/1.2818516.

A. J. Volponi. Foundation of Gas Path Analysis (Part I and II). von Karman Institute Lecture Series: Gas Turbine Condition Monitoring and Fault Diagnosis, January 2003.

A. J. Volponi. Gas turbine engine health management: Past, present, and future trends. Journal of Engineering for Gas Turbines and Power, 136(5), 2014. doi:10.1115/1.4026126. 
A. J. Volponi, H. DePold, R. Ganguli, and C. Daguang. The use of kalman filter and neural network methodologies in gas turbine performance diagnostics: A comparative study. Journal of Engineering for Gas Turbines and Power, 125(4):917-924, 2003. doi:10.1115/1.1419016.

J. W. Watts, T. E. Dwan, and C. G. Brockus. Optimal state-space control of a gas turbine engine. Journal of Engineering for Gas Turbines and Power, 114(4):763-767, 1992. doi:10.1115/1.2906654. URL http://link . aip.org/link/?GTP/114/763/1. 


\section{Appendix A}

\section{Mole/Mass Conversions}

\section{A.1 Mole/Mass Fraction Calculation}

Mole, and mass fraction conversion appears in different parts of the thesis. Here will these calculations be summarized.

\section{Mass and Mole Concentrations}

The mole concentration $\tilde{x}_{i}$ of species $i$ is defined

$$
\tilde{x}_{i}=\frac{n_{i}}{n}
$$

where $n_{i}$ is the number of mole of species $i$ and $n$ is the total number of mole in the mixture. The mass concentration $x_{i}$ of species $i$ is defined

$$
x_{i}=\frac{m_{i}}{m}
$$

where $m_{i}$ is the mass of species $i$ and $m$ is the total mass in the mixture.

Mole Mass

The mole mass of a mixture with mole concentration $\tilde{x} \in \mathbb{R}^{n}$, is defined as the sum of the elements

$$
M=\sum_{i} \mathrm{M}_{i} \tilde{x}_{i}
$$

where $M_{i}$ is the mole mass for species $i$, and the sum over all species is $\sum_{i} \tilde{x}_{i}=1$. The mole mass expressed in mass concentration $x \in \mathbb{R}^{n}$ can be written

$$
M=\sum_{i} M_{i} \frac{1 / M_{i}}{\sum_{j} x_{j} / M_{j}} x_{i}=\frac{\sum_{i} x_{i}}{\sum_{j} x_{j} / M_{j}}=\frac{1}{\sum_{j} x_{j} / M_{j}}
$$

where (A.6) is used. 
Mole fraction $\rightarrow$ Mass fraction

The conversion from mole fraction $\tilde{x}_{i}$ to mass fraction $x_{i}$ is

$$
x_{i}=\frac{m_{i}}{m}=\frac{M_{i} n_{i}}{\sum_{j} M_{j} n_{j}}=\frac{M_{i} n_{i}}{\sum_{j} M_{j} n_{j}} \cdot \frac{1 / n}{1 / n}=\frac{M_{i}}{\sum_{j} M_{j} \tilde{x}_{j}} \tilde{x}_{i}=\frac{M_{i}}{M} \tilde{x}_{i}
$$

where $m_{i}$ is the mass of species $i, n_{i}$ is the number of mole of species $i, m$ is the total mass, and $n$ is the total number of mole. In the last step, (A.3) is used.

Mass fraction $\rightarrow$ Mole fraction

The conversion from mass fraction $x_{i}$ to mole fraction $\tilde{x}_{i}$ is

$$
\tilde{x}_{i}=\frac{n_{i}}{n}=\frac{m_{i} / M_{i}}{\sum_{j} m_{j} / M_{j}} \cdot \frac{1 / m}{1 / m}=\frac{1 / M_{i}}{\sum_{j} x_{j} / M_{j}} x_{i}
$$

where $n_{i}$ is the number of moles of species $i$. Using (A.4), the mole fraction can be written

$$
\tilde{x}_{i}=\frac{1 / \mathrm{M}_{i}}{1 / \mathrm{M}} x_{i}
$$

\section{A.2 Stoichiometry Matrix Expressed in Mass}

The stoichiometry matrix, expressed in mole, repeated from (4.32) is:

$$
\tilde{S}=\left(\begin{array}{ccccc}
0 & 0 & 0 & 0 & 0 \\
1 & 2 & 3 & 1 & 0 \\
2 & 3 & 4 & 0 & 0 \\
0 & 0 & 0 & 0 & 1 \\
-2 & -3.5 & -5 & 0 & 0
\end{array}\right)
$$

where the rows represent the species: $\mathrm{Ar}, \mathrm{CO}_{2}, \mathrm{H}_{2} \mathrm{O}, \mathrm{N}_{2}$, and $\mathrm{O}_{2}$ according to the air vector $x_{a}$ in (4.30). The columns represent the species: $\mathrm{CH}_{4}, \mathrm{C}_{2} \mathrm{H}_{6}, \mathrm{C}_{3} \mathrm{H}_{8}, \mathrm{CO}_{2}$, and $\mathrm{N}_{2}$ according to the fuel vector $x_{f}$ in (4.30). Expression (A.5) can now be utilized for each matrix element to get:

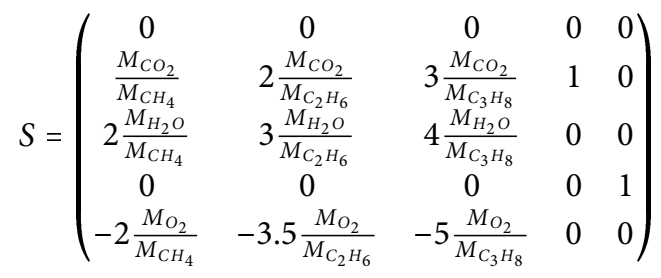

where the stoichiometric matrix now is expressed in masses instead of moles. 


\section{A.3 Determination of Stoichiometric Air/Fuel Ratio}

Reaction formula (4.34) represents a combustion, and is expressed in masses:

$$
m_{a} x_{a}+m_{f} x_{f} \rightarrow m_{a} x_{a}+m_{f} S x_{f}
$$

where $S$ is the stoichiometric matrix from (A.8). The stoichiometric air/fuel ratio appears when it is just enough oxygen in the reaction, i.e., the last row in (A.9) that represents oxygen is equal to zero and can be solved. The last line can be written:

$$
m_{a} x_{a, O_{2}}=m_{f}\left(2 \frac{M_{O_{2}}}{M_{C H_{4}}} x_{f, C H_{4}}+3.5 \frac{M_{O_{2}}}{M_{C_{2} H_{6}}} x_{f, C_{2} H_{6}}+5 \frac{M_{O_{2}}}{M_{C_{3} H_{8}}} x_{f, C_{3} H_{8}}\right)
$$

which can be rewritten in form:

$$
\frac{m_{a}}{m_{f}}=\frac{2 \frac{x_{f, \mathrm{CH}_{4}}}{M_{\mathrm{CH}_{4}}}+3.5 \frac{x_{f, \mathrm{C}_{2} \mathrm{H}_{6}}}{M_{\mathrm{C}_{2} \mathrm{H}_{6}}}+5 \frac{x_{f, \mathrm{C}_{3} \mathrm{H}_{8}}}{M_{\mathrm{C}_{3} \mathrm{H}_{8}}}}{\frac{x_{a, \mathrm{O}_{2}}}{M_{\mathrm{O}_{2}}}} \equiv\left(\frac{m_{a}}{m_{f}}\right)_{s}
$$

which is the definition of $(A / F)_{s}$. 



\section{Appendix B}

\section{Measurement Plots}

In this appendix, additional experimental data plots are viewed. These plots are the ambient temperature $T_{0}$, the ambient pressure $p_{0}$, the shaft speed of the gas generator $n_{G G}$ and the generated power by the external application $P$. 


\section{B.1 Ambient Temperature $T_{0}$}

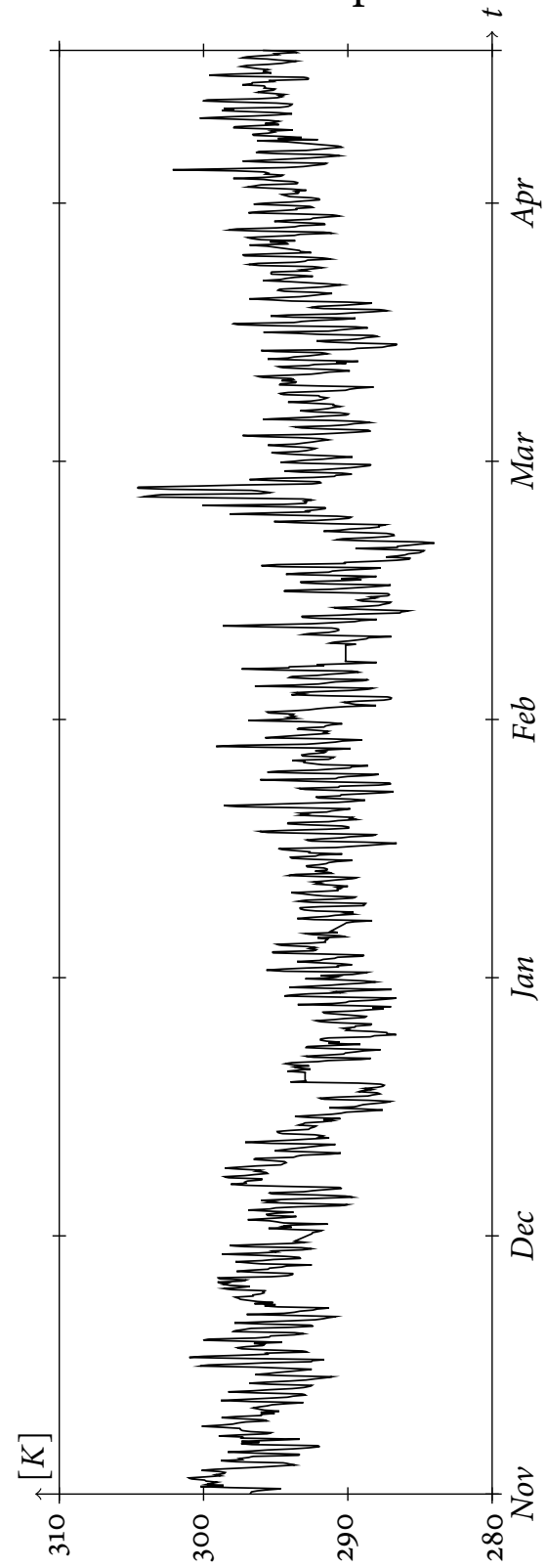

(a) $T_{0}$ for the first six-month period.

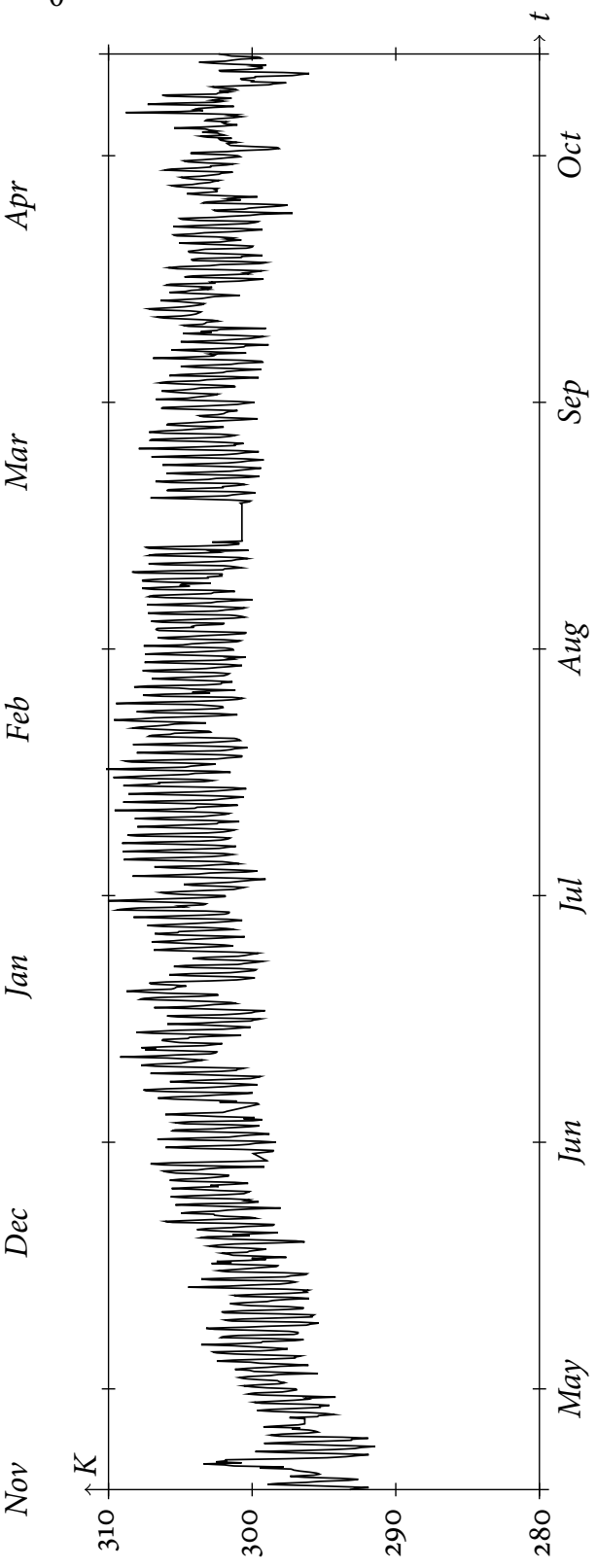

(b) $T_{0}$ for the second six-month period.

Figure B.1: In the figure, the ambient temperature $T_{0}$ is shown. The mean of the temperature varies according to winter and summer half year. In the end of February, the temperature rises for a couple of days, which affect the calculated measurement temperature deltas in, e.g., Figure 7.5. 


\section{B.2 Ambient pressure $p_{0}$}

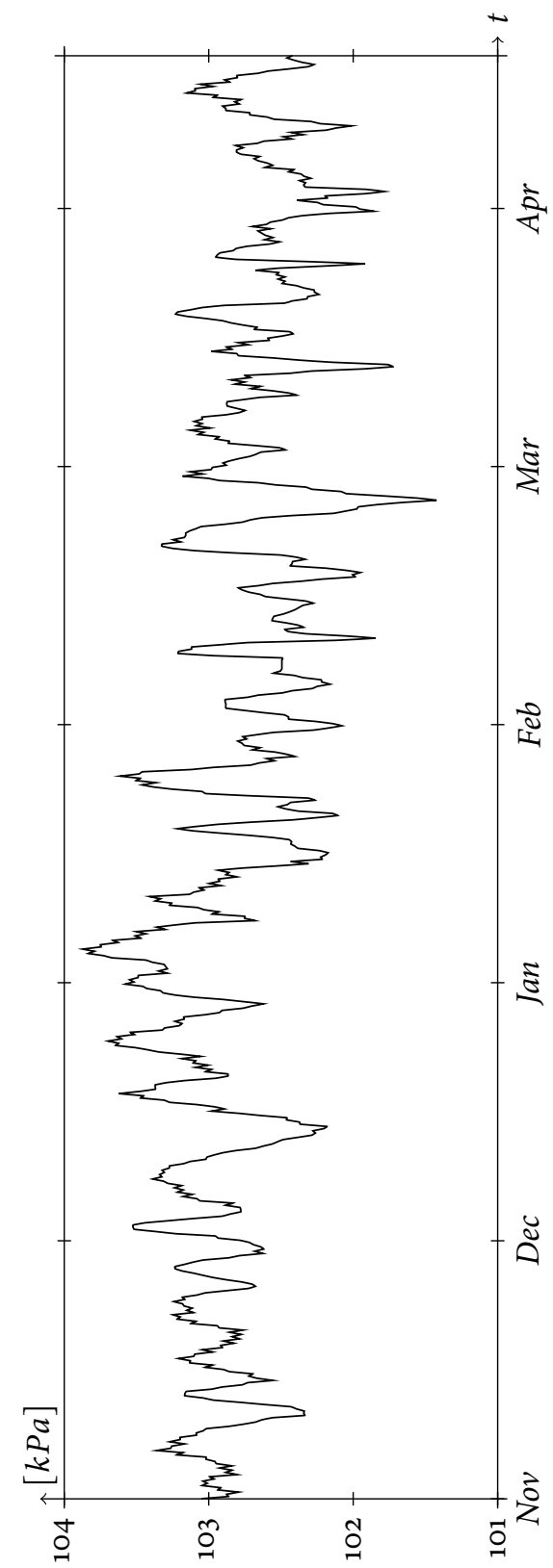

(a) $p_{0}$ for the first six-month period.

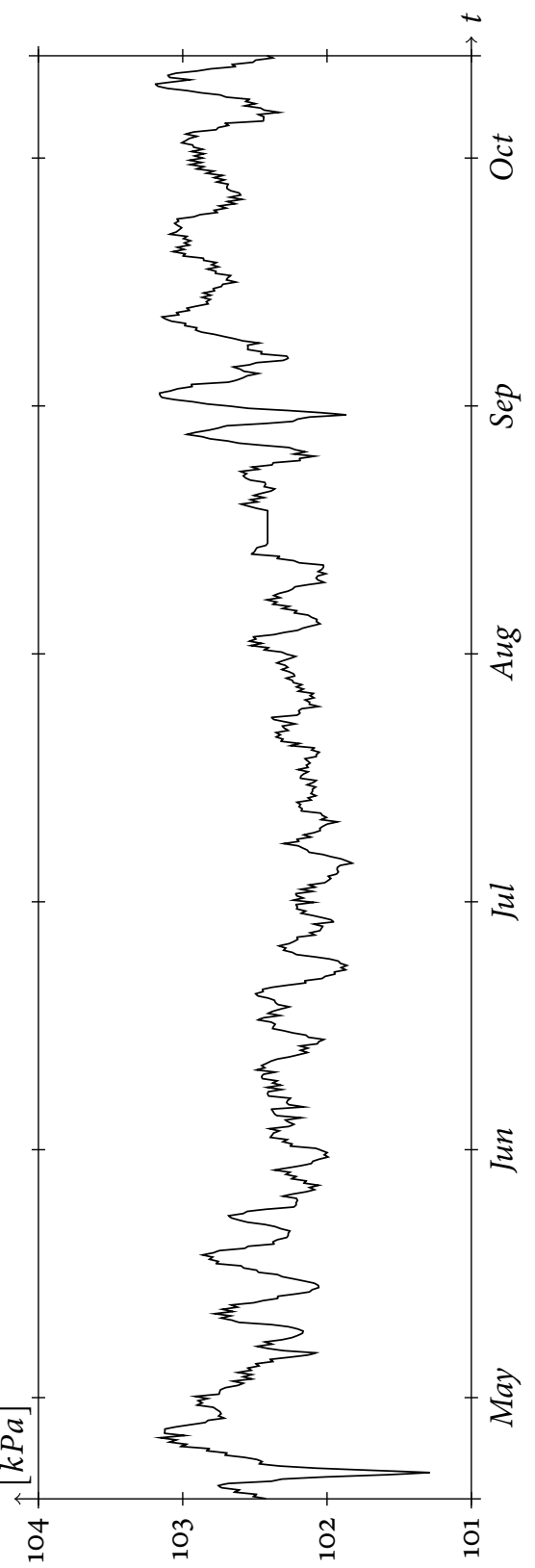

(b) $p_{0}$ for the second six-month period.

Figure B.2: Ambient pressure $p_{0}$ for one year of experimental data. 


\section{B.3 Shaft Speed $n_{C 1}$ of the Gas Generator}

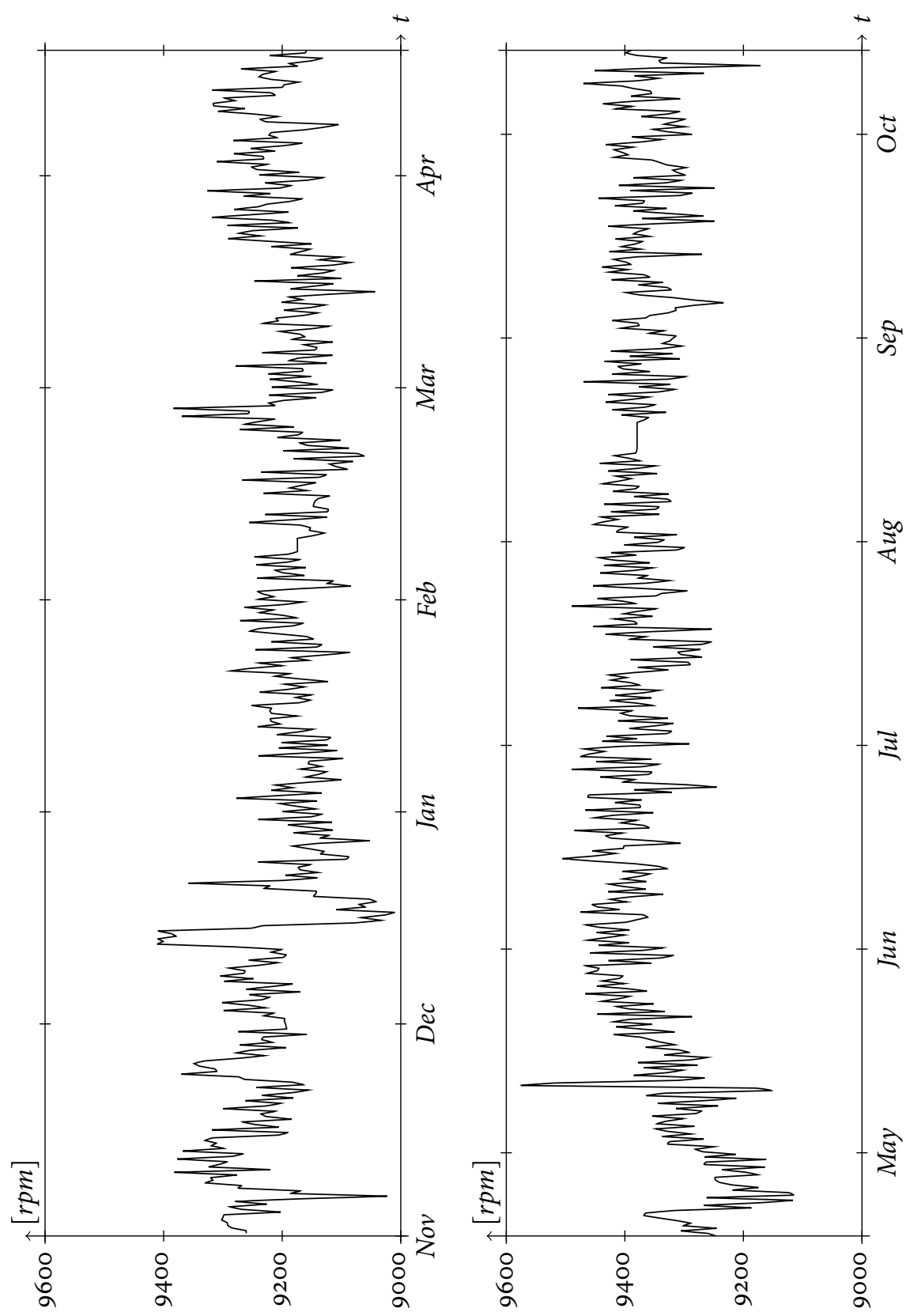

(a) $n_{C 1}$ for the first six-month period.

(b) $n_{C 1}$ for the second six-month period.

Figure B.3: Shaft speed $n_{G G}$ of the gas generator for one year of experimental data. 


\section{B.4 Generated Power $P_{A}$ by the Application}

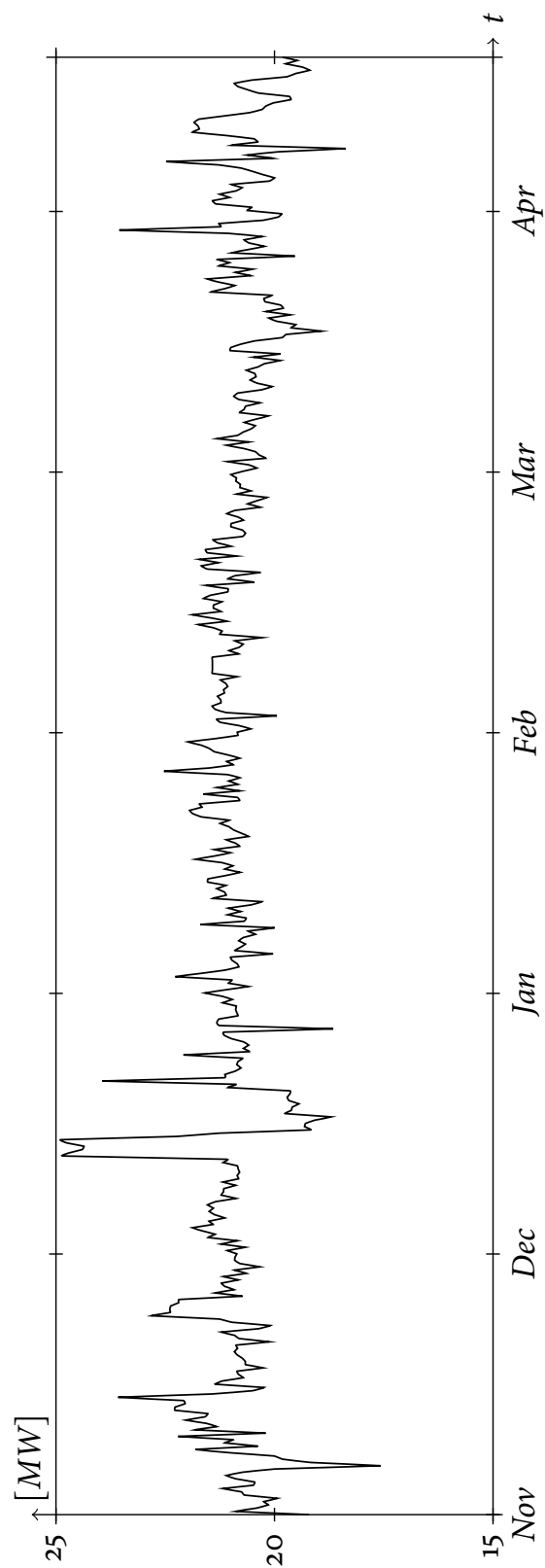

(a) $P_{A}$ for the first six-month period.

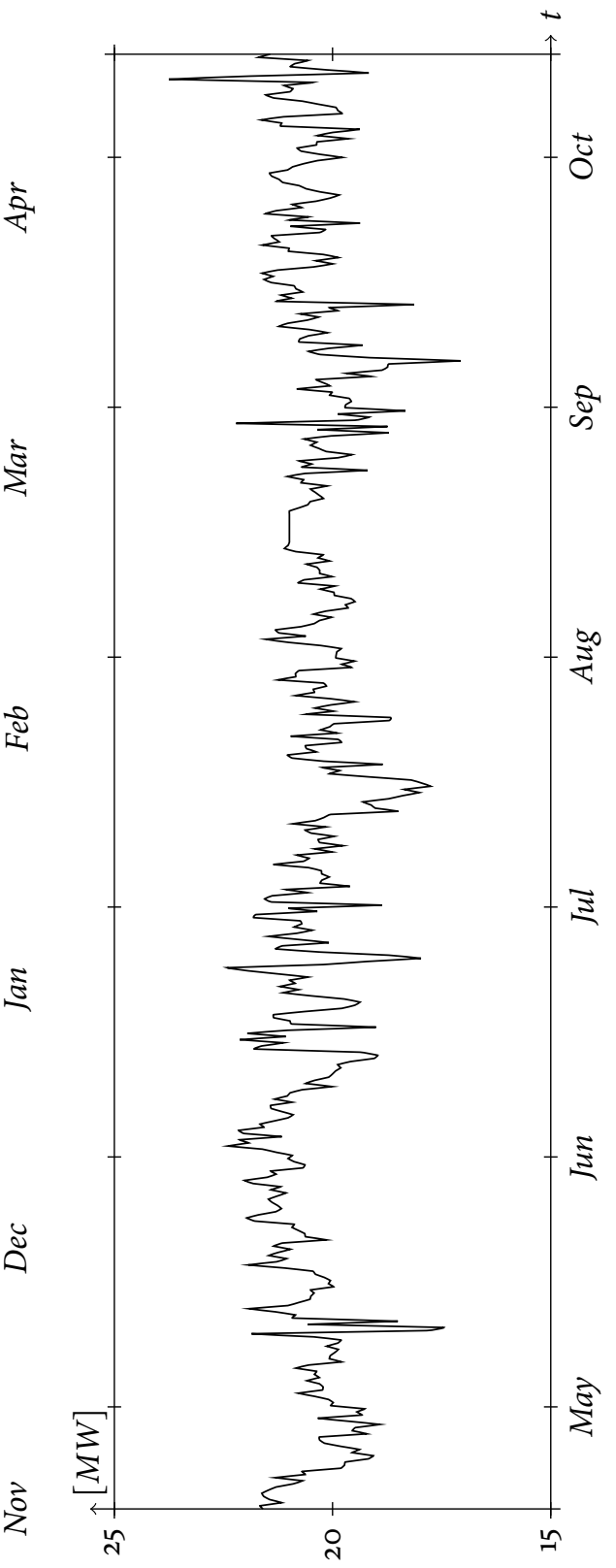

(b) $P_{A}$ for the second six-month period.

Figure B.4: Generated power $P_{A}$ by the application for one year of experimental data. 


\section{Linköping Studies in Science and Technology, Dissertations Division of Vehicular Systems Department of Electrical Engineering Linköping University}

No 1 Magnus Pettersson, Driveline Modeling and Control, 1997.

No 2 Lars Eriksson, Spark Advance Modeling and Control, 1999.

No 3 Mattias Nyberg, Model Based Fault Diagnosis: Methods, Theory, and Automotive Engine Applications, 1999.

No 4 Erik Frisk, Residual Generation for Fault Diagnosis, 2001.

No 5 Per Andersson, Air Charge Estimation in Turbocharged Spark Ignition Engines, 2005 .

No 6 Mattias Krysander, Design and Analysis of Diagnosis Systems Using Structural Methods, 2006.

No 7 Jonas Biteus, Fault Isolation in Distributed Embedded Systems, 2007.

No 8 Ylva Nilsson, Modelling for Fuel Optimal Control of a Variable Compression Engine, 2007.

No 9 Markus Klein, Single-Zone Cylinder Pressure Modeling and Estimation for Heat Release Analysis of SI Engines, 2007.

No 10 Anders Fröberg, Efficient Simulation and Optimal Control for Vehicle Propulsion, 2008.

No 11 Per Öberg, A DAE Formulation for Multi-Zone Thermodynamic Models and its Application to CVCP Engines, 2009.

No 12 Johan Wahlström, Control of EGR and VGT for Emission Control and Pumping Work Minimization in Diesel Engines, 2009.

No 13 Anna Pernestål, Probabilistic Fault Diagnosis with Automotive Applications, 2009.

No 14 Erik Hellström, Look-ahead Control of Heavy Vehicles, 2010.

No 15 Erik Höckerdal, Model Error Compensation in ODE and DAE Estimators with Automotive Engine Applications, 2011. 
No 16 Carl Svärd, Methods for Automated Design of Fault Detection and Isolation Systems with Automotive Applications, 2012.

No 17 Oskar Leufvén, Modeling for control of centrifugal compressors, 2013.

No 18 Christofer Sundström, Model Based Vehicle Level Diagnosis for Hybrid Electric Vehicles, 2014.

No 19 Andreas Thomasson, Modeling and control of actuators and co-surge in turbocharged engines, 2014. 